\title{
Rationalising individuality : the notion of individuality in biology, philosophy, (bio)ethics
}

Citation for published version (APA):

Salvi, M. (2002). Rationalising individuality : the notion of individuality in biology, philosophy, (bio)ethics.

[Doctoral Thesis, Maastricht University]. Universiteit Maastricht. https://doi.org/10.26481/dis.20020118ms

Document status and date:

Published: 01/01/2002

DOI:

10.26481/dis.20020118ms

Document Version:

Publisher's PDF, also known as Version of record

\section{Please check the document version of this publication:}

- A submitted manuscript is the version of the article upon submission and before peer-review. There can be important differences between the submitted version and the official published version of record.

People interested in the research are advised to contact the author for the final version of the publication, or visit the DOI to the publisher's website.

- The final author version and the galley proof are versions of the publication after peer review.

- The final published version features the final layout of the paper including the volume, issue and page numbers.

Link to publication

\footnotetext{
General rights rights.

- You may freely distribute the URL identifying the publication in the public portal. please follow below link for the End User Agreement:

www.umlib.nl/taverne-license

Take down policy

If you believe that this document breaches copyright please contact us at:

repository@maastrichtuniversity.nl

providing details and we will investigate your claim.
}

Copyright and moral rights for the publications made accessible in the public portal are retained by the authors and/or other copyright owners and it is a condition of accessing publications that users recognise and abide by the legal requirements associated with these

- Users may download and print one copy of any publication from the public portal for the purpose of private study or research.

- You may not further distribute the material or use it for any profit-making activity or commercial gain

If the publication is distributed under the terms of Article $25 \mathrm{fa}$ of the Dutch Copyright Act, indicated by the "Taverne" license above, 


\section{Rationalising Individuality.}

The notion of individuality in biology, philosophy, (bio)ethics

\section{PROEFSCHRIFT}

ter verkrijging van de graad van doctor aan de Universiteit

Maastricht,

op gezag van de Rector Magnificus, Prof. Dr. A.C.

Nieuwenhuijzen Kruseman volgens het besluit van het College van Decanen, in het openbaar te verdedigen op vrijdag 18 januari 2002 om 12.00 uur

$$
\text { door }
$$

Maurizio Salvi

Geboren op 19 April 1967 te Tropea 


\section{Promotores:}

Prof. Dr. R.H.J. ter Meulen

Prof. Dr. W. Callebaut, (Limburgs Universitair Centrum, Diepenbeek, België)

Prof. Dr. S. E. Cuypers, (Katholieke Universiteit Leuven, België)

\section{Beoordelingscommissie:}

Prof. Dr. R.Vos (voorzitter)

Prof. Dr. E. van Leeuwen (Vrije Universiteit Amsterdam)

Prof. Dr. H. Pott (Erasmus Universiteit Rotterdam)

Dr. H. Smit

Prof. Dr. W.J. van der Steen (Vrije Universiteit Amsterdam) 


\section{PREFACE}

I began to work on the notion of individuality in both biology and theoretical medicine in Rome, 1995, where I was working as researcher at Institute of Bioethics, University of Rome 'La Sapienza'. During my research work I quickly realised that I had to approach the issue from an intemational perspective. I then applied for an individual research fellowship to the European Commission. I got the fellowship and I joined the University of Maastricht where I begun my PhD research work (1996). Prof.dr. Gerard DeVries, Dean of the Faculty of Art and Culture at that time, was the responsible of my research project. Prof.dr. Werner Callebaut was the supervisor of my research.

Unfortunately in 1997 both Prof. De Vries and Prof. Callebaut left the University of Maastricht (the first one got a position at the University of Amsterdam and the second one at the Konrad Lorenz Institute and the University of Vienna). Prof. Callebaut agreed to continue to act as supervisor of my research from Vienna. I then asked Prof.dr. Ruud ter Meulen to supervise my thesis (1998), with the agreement of the Dean of the Faculty of Art and Culture, Prof. Dr. Wybe Bijker. Professor Ter Meulen kindly accepted my proposal on the condition that Prof. Callebaut would continue the supervising of the sections on the theoretical biology. Later on Prof.dr. Stefan Cuypers of the Catholic University of Louvain agreed to join the team of promotors, particularly to supervise the philosophical sections on personal identity. Prof. Ter Meulen was supervising the section on bioethics and was responsible for the over-all supervision of the thesis.

My research contract with the EC finished in 1998 and then I continued my research activities at the International Forum of Bio-

\footnotetext{
'BIOTECH II-, Research topic: Germ line gene transformation of the transgenic mouse Contract Nr: .BIO4-CT96-5075
} 
philosophy centre (IFB, KU Leuven), working on a research project ${ }^{2}$ funded by the Flemish Institute of Biotechnology (VIB). The research activities done with IFB were functional to develop the chapters of the thesis dealing with ethics of human genetics.

In parallel, in 1998 I got a Scientific Officer (END) position at the European Commission (DG Research). I was appointed as responsible officer for bioethics research in Europe. This job has allowed me to have direct access to ongoing discussions in Europe about new discoveries of the natural sciences and medicine as well as to address the regulatory aspects of biotechnology.

From 1998 to 2001 the manuscript has been finalised. The finalisation of the manuscript was not easy due to a number of factors: (a) the manuscript had to be revised and approved by three people from different countries, (b) the stylistic and linguistic revisions of the manuscript had been performed outside Europe (USA, Australia) and (c) a number of scientific discoveries that were relevant for my research topic had to be critically added to the manuscript (from cloning to the human genome sequencing). But finally the thesis has been finalised and approved by the promoters and the reading committee in November 2001.

I wish to express my gratefulness to a number of people that were directly or indirectly involved in the preparation of this thesis. From a scientific point of view, apart from the marvellous job done by my promoters (and I wish to stress that because of the circumstances described above without Werner, Ruud and Stephan this thesis would never have materialised), I had the chance to discuss several topics of this dissertation with numerous experts. Only to quote some of them: Prof. Harris (UK), Prof. McGee (USA), Prof. Macer (Japan), Prof. Lecaldano (IT), Prof. Van Steendam (B), Prof, Verhog H. (NL), Prof. Berlinguer (IT), Prof. Hottois (B), Prof. Oliverio (IT), Prof. Hermeren (SW), Prof. Rodotà (IT), Prof. Casabona (E), Prof. Schroten (NL), Prof. Gagliasso (IT), Prof.

\footnotetext{
${ }^{2}$ Research project "Towards a multi-actor social mapping of ethical aspects of Human Germline Gene Therapy. The case of cancer and neuropsychiatric diseases"
} 
Daniels (NZ), Dr. Kishore (India), Prof. De Castro (Philippines). From all of them I got suggestions that helped me to clarify my enthusiastic but sometimes confused ideas.

But also I do want to express my gratefulness to my parents that supported me during my university training. I am deeply indebted to both my wife Silvia and my son Luca ('bello di papà'). Silvia followed me in this adventure in the North of Europe and had to undergo both my difficult times (scientific and professional difficulties) and the unavoidable repercussions they had in my mood. Luca simply cnlightened my life with his birth. To Silvia and Luca this thesis is dedicated. It is difficult for the brain to work properly when the heart does not receive love. I never missed Love from Silvia and Luca. 


\section{Contents}

Preface, pp. I-II

\section{Part One: Biological Problems}

I.1. Organisms and Identities, pp. 1-26

1.1 Classification Systems; 1.2. The Concepts of: Unity and Whole; 1.3 Biological Identity; 1.4. Unifying Variable and Heterogeneous Systems; 1.5. Organismic Biology; 1.6. Organisms as Organic Units: 1.7. Why isn't a Cellular Culture an Independent Identity?

\section{I.2 Genetic Identity, pp. 27-48}

2.1. Biological Determinism: The Theory-of-the-Farm (TF); 2.2 The Paradox of Monozygotic Twins; 2.3. The 'Talkative DNA' Theory; 2.4 Genetic Plasticity; 2.5 Philosophical Implications; 2.6 The Unification of the Organic Unity

I.3 Plasticity through time, pp. 49-63

3.1 Taxonomic Problems: How To Classify Living Beings; 3.2. The Species Concept in Biology; 3.3. Reproduction in Systematic Biology; 3.4. Analytical Approach to the Species; 3.5 Species as an Organic Unity

1.4. The Organisms Environment Dialogue and the Organic Unity Theory, pp. 64-77

4.I. Biological Paradigms in Evolutionary Microbiology; 4.2. Mathematical Models in Biology; 4.3 Dialoguing with Nature; 4.4. Organic Unity as a Dialogue Dimension

1.5 Neural physiology and Self-Synthesis, pp. 78-87

5.1. How does the Brain Work? 5.2 Quantum Theory and Neural Interconnections; 5.3. Quantum Physics and Neuroscience; 5.4. Is Neural physics Really Interested in Explaining Consciousness?

I.6. The Mind: A Biological Perspective, pp. 88-114

6.1. Is the Mind a Computer? 6.2. Hardware or Software: What's in the Human Head? 6.3. The Role of Memory in the Mind; 6.4. Self-Awarenes. and the Unity of Consciousness: the Biological Perspective; 6.5. Self- 
Synthesis and the Unity of Consciousness: the Philosophical Perspective; 6.6. Self Reflexivity and Self Awareness; 6.7. Philosophy and Neurology

I.7 The Organic Unity Theory, and the Variability of Our Existence, pp. 115-128

7.1. Persistence of Individuals; 7.2. Psychological Continuity; 7.3. Closest Continuers; 7.4. Conclusion: Diachronicity and Complexity of Individuals.

1.8. Analogous Processes in the Unification of the Organic Unity, pp. 129-138

8.1 What is Analogy? 8.2. Analogy and Self-synthesis; 8.3. Biological Features of the Self-Synthesis; 8.4. Analogy and Self-Unification

1.9. The Organic Unity 'Species' and its Unification, pp. 139-154

9.1. Evolution and Rationality; 9.2. The Role of Rationality in Evolutionary Dynamics; 9.3. Culture as a Fitness Vector; 9.4. Rationality as a Species Unifying Property; 9.5. Culture and the Emergence of a Species-Specific Self-Consciousness

Conclusions: Biological Problems, pp. 155-158

Section Two: Shaping Individuality.

II.1. Conceptual Premises, pp. 159-166

1. 1. The Responsibility of Science in the Field of Biotechnology, 1. 2. The Responsibility of Science in the Field of Biotechnology, 1.3 Ethics of Science, Science of Ethics, 1.4. The Organic Unity Theory as a model to use in bioethics.

11.2. Genetics' Dreams, pp. 167-182

2.1 Is Gene Therapy the Future of Biotechnology? 2.2 Dreams of Genetics;

2.3 Treating or Enhancing? 2.4 Bioengineering and public health; 2.5

Consequences of Genetic Determinism in Human Genetics; 2.6 Conclusions.

II.3. Shaping Individuality: Human Inheritable Germ Line Gene Modification, pp. 183-197

3.1 Bioethics and Humam Inheritable Germ Line Gene Modification (HIGM); 3. 2. Pre-embryo's identity; 3.3. Human inheritable germ line 
gene modification and the Individual's Identity; 3.4. The Persistence of genetically modified embryos and the Closest Continuer Scheme; 3.5 Conclusions

II.4. Transforming Animal Species: the Case of 'Oncomouse', pp. 198210

4.1. Transgenic Animals; 4.2. Problematic implications of transgenic: animals production; 4.3. Why choose the example of 'oncomouse'? 4.4. The Moral Implications of the oncomouse Production; 4.5. Ethical Implications arising from the production of the Oncomouse; 4.6 Conclusions.

II.5. Animal cloning, pp. 211-220

5.1 Dolly Superstar; 5.2 Why cloning animals? 5.3 Biological implications of cloning; 5.4. Animal Cloning.

I1.6 Human Reproductive Cloning, pp. 221-235

6.1 Human Reproductive Cloning; 6.2 Cloning and Personal ldentity problems; 6.3 Conclusions.

Conclusions: Shaping Individuality, pp. 239-238

Epilogue, pp. 239-245

Bibliography, pp. 246-278

Summary and CV, pp. 279-290 
To Silvia and Luca this thesis is dedicated. It is difficult for the brain to work properly when the heart does not receive love. I never missed Love from Silvia and Luca. 


\section{Preface.}

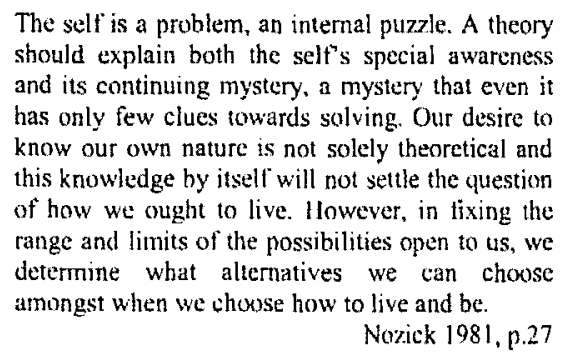

This dissertation addresses issues related to the notion of individuality in both theoretical biology and philosophy ${ }^{1}$. The topic is not new. Scientists, philosophers, and, more recently, bioethicists have dealt (and are dealing) with this dilemma. Nevertheless, to date, the debate around the viability of the notion of individuality is not concluded. The term 'individual' has often been used broadly, as an 'umbrella' word or referred to a specific scientific branch (from genetics to neurosciences, from analytic philosophy to applied ethics). This has induced experts of different disciplines to posit identity theories based on one-dimensional explanations of 'individuality'. There are theories advocating genctic identity, or bodily identity, or psychological identity, or ontological identity and so on. These views approach individuality within a specific scientific discipline (from genetics to neuroscience; from philosophy of mind to metaphysics). They segment the complexity of living beings in sets of simplex systems, and successively deduce the features and behaviours of complex systems from the simplex systems'. Clearly, these claims refer to the subject's features taken into account and often collapse when used to explain other features of living beings than the analysed ones. Then, we see a paradoxical phenomenon in which, for example, identity views based on individuals' mental capacities cannot explain the existence of living beings during embryogenesis. Or theories thinking of Life as a chemo/physical phenomenon that result completely inadequate to explain the qualitative features of living beings (behaviours, mental phenomena). Why we do not address individuality as a unitary puzzle? Is it possible to advocate a theory on individuality able to explore both the physical and phenomenological nature of individuals? 
So far two methodologies have been used to explore the problem of individuality: 1) an approach which thinks of individuality as a complex problem to be explained via hard sciences; 2) another one which thinks of individuality as a complex and synergetic problem that cannot be elucidated via reductionism but needs further explanations on the phenomenological nature of living beings. Both these methods think of individuals in a different way. The first one claims that the physical nature of organic systems is understandable, because Life is a physicochemical phenomenon. The second one says that Life cannot be physically explained since new features of organic systems emerge from their unified complexity. However, since Life is both a physical and phenomenological phenomenon, how can we separate these organisms' features? Is the problem of individuality a theoretical or practical dilemma?

This disseration deals with the above mentioned issues. I will claim that the notion of individuality cannot be investigated without bringing together two lines of research: biology and philosophy. I will propose a methodology, according to which the identity problem is seen as a pragmatic problem that needs interdisciplinary analyses. This is for a number of reasons. First, Life is a complex synergetic phenomenon whose understanding cannot be taken only via a specific expertise (genetics, neuroscience, genomics, etc.). Second, the use of new technologies has caused a paradoxical phenomenon according to which on the one hand, we can manipulate the biological existence of future-born subjects or 'manufacture' artificial organisms (let us think of transgenic animals, GMOs, bioreactors, chimeras, hybrids, etc.), on the other hand we do not know what the existence of an individual is. In the light of this, I will explore the biological features of organic systems and successively I will claim a model, the organic unity model, which may solve a number of identity dilemmas.

\footnotetext{
' As I have said, my interdisciplinnry study about the notion of individuality refers to coustless scientifie disciplines. Then. in my dissertation I have used a linguage deprived of jargons (when possible). When it was impossible to use specialised words, I have tried to explain the meaning of the used terms. Some expert may feel tired of these continuous explanations.

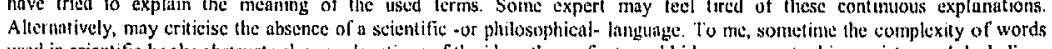
Alternatively, may critieise the absence of a scientific -or philosiphical- langunge. "T'u me, sometime the complexily of words
used in scientific books obstructs clear explanations of the ideas they refer to and hides a comeeptual inconsistency. I do believe that the first gool of a hook is to communicate of ideas. Then, I have closen the use of a language accessible to a broad that the first gont of a hook is to comnunticate of ideas. Then, I have
audience. When my aim has been nol achievect. I apologise for my failure.
} 


\section{I.1. Organisms and Identities}

Merriam-Webster's Collegiate Dictionary defines an organism as:

1: a complex structure of interdependent and subordinate elements whose relations and properties are largely determined by their function in the whole.

2: an individual constituted to carry on the activities of life by means of organs separate in function but mutually dependent: a living being.

At first glance this definition does not appear problematic. We know who we are. I know who I am. We would be unsatisfied with our definition of 'individual existence,' 'Self, or 'personal identity'. I will use different terms, such as 'identity', 'individuality', 'organism', 'Self', to signify the same concept: the union of elements, such as body, emotions, thoughts, and feelings, which constitute the individuality and identity of an organism. The problems of individuality embody and exemplify the difficulty of explaining the complexity of natural phenomena.'

Philosophers usually make a distinction among the notions of 'individuality', 'identity', and 'self. For example B. Williams (1973) stressed the conceptual difference between the notions of identity and individuality. He claimed that bodily continuity of individuals was a pre-requisite and a key element to take into account in identity theories. Other authors have endorsed theories based on other basic features of individuality, such as brain continuity (Nagel, 1973), psychological continuity (Parfit, 1984; Nozick, 1981), psychological criteria (by advocating modern interpretations of Hume and Locke - see Di Francesco 1999), and so on. In bioethics, a number of authors have advocated personhood theories (from Sgreccia, 1984 to Harris, 1984) to explore the notion of individuality within a moral framework. The use of these notions is also common in biology. For example immunologists (see Tauber, 1992) and cognitivists (see autopoiesis theory) have used the notion of 'Self', 'individuality', 'identity' to label organisms.

In this dissertation I will address the problem of individuality as a complex topic that needs to be depicted through different media, from biology to neuroscience, from philosophy to ethics. Methodologically 1 will face the complexity of the identity problem through the various scientific disciplines 
that have contributed to the definition of Life (from genetics to biology, from neuroscience to psychology). I will claim that a theory of individuality has to face, and possibly explain, both the biological and phenomenological nature of living beings. This means that I will not make a distinction between 'self' and 'body', 'identity' and 'individuality' because this thesis aims to show that these terms do refer to the same entity: the Living being. In order to avoid confusions and misunderstanding, I invite my readers to be patient, then, with my terminology and not to think that I am not aware of the debate about the notion of identity in the history of philosophy (from Plato to Leibniz, from Kant to Nozick).

What elements explain my presence in this world? What is identity? Is my identity completely restricted to my body, or is it linked to external elements? Am I a set of mental states or a single physical object? These questions illuminate some potential problems in attempting to conceptualise identity. How may we begin to define identity is the fundamental inquiry of philosophy. Clarifying what identity ${ }^{2}$ is means to elucidate the meaning of our existence as specific entities, and to approach the problem of internal coherence within the separate phases of our lives. Am I the same person today as I was at age 10 ? When we can distinguish properties that seem capable of explaining our existence and continuity, we can begin to think of our lives in terms of a unitary and coherent process. An analysis of concepts of individuality in both biology and philosophy can help us to investigate questions that probe the very meaning of our lives. We can look for part of the answer to the question 'Who am I?'.

\subsection{Classification Systems}

When I see an object, 1 ascribe an identity to it. For example, I may see a stone and remark, 'This is a stone'. If the stone has any particular features (for example, smearing colours, a jagged edge, one smooth side) I classify it as a stone and later as 'that' stone. Through the first classification process we say that an object belongs to a specific linguistic class (the object falls into the classification of stones). The second process leads us to say that stone is a particular stone. The identity of a stone is its presence as a specific thing, which possesses specific features. These features allow an observer to differentiate any old stone from 'that' stone with its smooth back and clay-coloured belly which has an identity of its own ${ }^{3}$. Why do we even think of that stone as a unitary entity (one entire stone) when it is 
composed of innumerable atoms? What leads us to think of the identity of an object as a unity?

If I think of the stone's atomic configuration, I know that this unitary object is composed by a number of constituents (atoms). The stone is a homogenous locus of heterogeneous constituents. Its constituents have a specific atomic structure as well as a spatiotemporal relation. They share a spatiotemporal dimension. Since atoms have this common spatiotemporal connotation they constitute a unitary entity and have a common internal interrelation. The stone's physical existence, therefore, refers to its observer and their applied classification criterion, but also on 'that' stone in itself. This means that a stone's existence is independent of its observer (as stones exist without observers). At the same time, its identity is observerdependent (the phenomenological nature of experienced objects ${ }^{4}$ ). The identity of an object refers to a classification system that is simultaneously external and internal in relation to the object being investigated.

As I have already said, the stone is a set of spatiotemporally joined atoms. The unitary meaning of its existence refers to our classification criteria (a stone does not have self-referential properties ${ }^{5}$ ). Since we approach objects using perception, a stone's structural complexity becomes insignificant in our classification system, because we perceive it at its macro-level. We thus attribute a unitary meaning that is independent of the structurally integrated micro-complexity of the stone. We classify it as a unity at its macrocomplexity, because our perceptual mechanisms are limited to 'that' dimension. Nevertheless, since the stone's macro-complexity refers to its micro-complexity, we need to analyse the nature of this relation, and to study the causative factors that unify a stone's constituents (atoms and molecules) in a unitary dimension. We therefore must single out a cohesive property that unifies an object in itself.

In classification systems, the first relation of cohesiveness is the spatiotemporal relationship within an object's constituents (s-relation). The second relation of cohesiveness is the causal link within its constituents. An object thus is a spatiotemporally bounded entity displaying internal organisation and cohesion. In inorganic systems, deprived of the possibilities of reproduction as they are, the spatiotemporally bounded constituents have another feature: they are similar. This condition clusters an object's constituents. In fact, a stone's atoms have a 'similar' structure when viewed from a physicochemical perspective. This allows me to assert that the reiation of state within a stone's atoms is reinforced by a causal element: similarity among an object's constituents. This is important 
because, while being a link between the object's constituents, the s-relation does not deal with the constitutive features of a stone's constituents; it simply shows us 'how' these elements are interconnected, but not 'why' they can be contingent on 'that' stone and not a tree. We need to approach the identity dilemma by looking for two identity features: the s-relation and the maximum similarity among the object's constituents.

We will see that the s-relation identifies a spatiotemporal relation, the maximal similarity condition links the constituents of a set in a causal sense and in doing so defines individual identities within a class of elements ${ }^{6}$.

\subsection{The Concepts of: Unity and Whole}

Let us consider an elementary example of classification mechanisms: the classification of sets of points in a surface as advocated by Robert Nozick (1981:85). Let us consider the following figure.

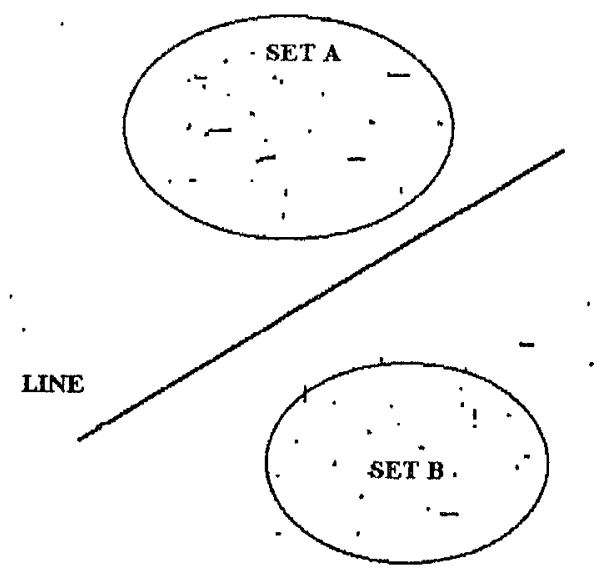

The simplest procedure of classification is the grouping of points in a surface through a relationship based on proximity. Let us think of a straight line darting across a surface. The line delineates two sections of a surface. In both surfaces there are two sets of points. 1 classify them by default as part of the same set because of their proximity. There simply are no other points in the class closer to one of them than each and every other thing in 
the class (Nozick 198185). My classification reference is the line and the distance of various points in regard to that line. Through this procedure of classification, I group points in two classes (two wholes) in respect to a common reference (in this case a line). The distance of points from the line is the classification condition of their appendage (or lack of) to Set A or Set $B$. This is an example of the s-relation clustering points in unitary classes.

Since the relation of internal proximity is our classification system, it follows that the s-relation is the causal element by which points are (grouped and) classified into sets. However, this clustering property does exactly explain how we can recognise sets on their own merits, independent from their spatial interdependency. It explains the spatiotemporal relation that links the elements of a set, but it is silent when asked to explain their qualitative or causative interconnection. If we do not specify these parameters, we can group points in two sets (Set A and Set B), but we have no information concerning their properties or peculiarities (for example, the number of points, their colour, form, and so on). The s-relation thus has a basic value in our classification system, but only separates the unitary nature of composite classes. If the line disappears, we cannot group points into sets $A$ and $B$, and yet these points remain. How can we then classify them?

We can classify them as 'sets of points' because they possess a common feature (they are points) and an internal similarity that clusters them in specific classes (suppose $A$ 's points are red and B's points are green). This means that a relation of state allows us to group things in unitary classes, but this criterion of classification needs to be reinforced by further properties that internally link elements of a class (cells, atoms, books, and so on). The second classification property serves as the subject's similarity among its constituents. ("The principle of clustering things together in a classification maximizes within-class similarity while also maximizing between classes dissimilarity" Nozick, ibid, p. 86,) By emphasising the similarity within points we classify them as certain classes, or as constituents of a global entity that is in effect formed by them. The relation of similarity clusters members of a class from inside that same class.

The case of points in a surface is a simplistic example of a classification system. Other examples depict a higher complexity. Take the case of a drop of water. Atoms that are linked by a s-relation construct the drop. At the same time, these atoms possess a common property: an internal similarity so to speak (atomic configuration of $\mathrm{H}_{2} \mathrm{O}$ molecules). The s-relation says that a drop of water is a unit because its molecules share a spatiotemporal system. The classification criterion is reinforced by atomic similarity between water 
constituents. This means that qualitative features of the unit drop increase its unitary meaning.

As a consequence of this property, when the drop falls into a glass of water its identity is absorbed by a global entity - 'the water' - since both maximum similarity and s-relation parameters join the drop's molecules with other molecules contained in the glass. If, on the other hand, a drop of water falls into a glass containing oil, the difference between the atomic configuration of these liquids avoids the fusion of water and oil, and thus the conflation of the identity of water with that of oil. Even if liquids have a common spatiotemporal relationship (they are in the same glass), they still maintain a structural diversity that defines them in terms of different units.

Considering this preliminary discussion, we acknowledge that the concept of identity appears to be quite complex. We can talk about the identity of something once we have established a cohesive s-relation and an internal similarity (for example a set of chemical and physical features, such as colour, form, density, and so on). It is important to observe that classification systems respect various specific conditions. One is the property to have an internal s-relation within an object's constituents. This condition defines the concept of unity: a unity is a set of constituents that are spatiotemporally bounded. Unity is a state of being glued together by a shared spatiotemporal relationship and/or a common similarity.

At this point I have analysed some initial identity problems in objects that have a unitary and stable structure. In Nature we could find a large number of objects that have integrated heterogeneity and structural variability. A sand dune is a perfect exannle. ${ }^{7}$ We think of its identity as a specific unity, but a sand dune is composed of a variable number of individual grains. It is a variable whole of grains that maintain a specific srelation in a spatiotemporal system. We think of it as a unitary entity independent of its composite and variable nature. The numerical variability of its elements does not influence the meaning of its unitary nature. As such, we consider the dune to be a unity that continues independently from its structural variability. This means that a relationship between the unity of objects that have this composite nature and their constituents does exist. Why do we attribute a unitary nature to a sand dune when its constituents vary $^{\gamma}$ ? In the course of seconds, weeks, and years, the wind disperses some grains and adds others. The constitution of the dune varies while its individual grains combine to form a whole. Nevertheless, its global identity continues independently of this structural variability (Wimsatt, 1972). In realising this, we recognise two additional problems: 
1. When does a group of individual sand grains become a single dune?

2. Is a dune's identity-persistence the same, even though the individual grains continuously change?

The first question refers to the unity concept, the second on the whole concept. The set of grains is a unity because it has a common spatiotemporal relation. Simultaneously, these grains form a whole because they constitute a composite entity whose identity does not change if its constituents (grains) change as time passes. The variability of this heterogeneous identity does not produce any direct consequences to the sand dune's continuity.

A unity is a set of elements that maintains a fixed spatiotemporal interconnection. A whole is a product of sub-units ${ }^{9}$ that compose a unitary entity independent from their constitutive and qualitative changes.

The sand dune is a 'whole-unity' because its constituents (grains) have an internal relation that sculpts them into a unitary entity whose identity is independent of its constitutive changes. The concept of a whole allows us, then, to think of an entity in its continuity at different temporal stages. We can therefore approach problems of identity-persistence through the wholeunity concept. Why? Because organic systems are heterogeneous variable identities that continuously (qualitatively and structurally) vary their cells, fibres, organs or tissues.

\subsection{Biological Identity}

The analysis of a dune's identity is concurrent to the problem of defining identity in organisms, because a dune continuously changes its grains in the same fashion that an organism changes its cells. Many examples of massive change within an organism's constituents exist in biology. An example of such a phenomenon is the bones of a mammal's skull. (Wagner, 1994) The embryogenetic stages of the foetal development of a sheep show a complete substitution of the peripheral material of the foetal parietal bone. The structural biological identity is continuously challenged by abundant biological phenomena such as embryogenesis, organ growth, metabolism, and so forth. This suggests that the variability of 
the structural identity of an organism is a specific property of organic matter, and encourages us to question the problem of biological identitypersistence despite its continuous qualitative-structural transformation. An extremely high level of complexity and heterogeneity reinforces this structural variability in organic systems. This complexity connotes every hierarchical level of organisms from the nucleus to the cell, from the organ to the individual, from species to ecosystem. Nevertheless, a hierarchical organisation of living creatures maintains cohesive factors that cluster the heterogeneity of living systems in a unitary entity. Each level of complexity is compounded into new entities at the next level of complexity (Mayr 1988). The coexistence and cohesive integration of systems cause the 'emergence' of characters that cannot be deduced from the knowledge of an organism's constituents ${ }^{10}$ (Egidi 1987). Whitehead in Science and the Modern World defined an organism as follows:

"The organism is a unit of emergent value, a real fusion of the characters of eternal objects, emerging for its own sake. Thus in the process of analysing the character of nature in itself, we find that the cmergence of organisms depends on a selective activity which is akin to purpose" (Whitehead 56:151-2)

To gain a greater awareness of the complexity of living beings' individuality, it would help to begin analysing some of the peculiar features of biological individualities. The first property of biological individuality is a continuous structural variability. This dictates that if an organism's identity is explained by its own constitutive elements, then we are obliged to continuously redefine it when a component of that organism's body is altered. Biological identity should continuously be redefined depending on cellular changes. Nevertheless, as a dune's identity continues independently from structural changes over a period of time, an organism's identity continues through time regardless of its qualitative-structural changes. Biological individuality is thus based on mechanisms of continuity that are independent from an organism's biological variability (cells, organs, systems). This property is explained by an organic system's specific property: biological systems act as wholes, and cluster the heterogeneity of their structural identity in a homogeneous and cohesive entity ${ }^{1 !}$.

Again we seem to have attempts at organisation that are plagued by complexity. Organisms are loci of integrated complexity, with features undetectable from knowledge only of their constituents. However their constituents continuously change without causing significant consequences 
in an organism's continuity ${ }^{12}$. This plethora of problems is manifest in the question, 'What is biological identity?'.

Biologically speaking, I assign an organism $(X)$ an identity because it has physical and biological features denoting it as a unitary and unique individual (genome, phenotypic characters, and bodily dimensions). An organism is a whole-unity because its constituents are spatiotemporally interrelated (r-related), and because they continuously change. When we stress the composite nature of organic systems, we have to solve the possibility of explaining an organism's identity through an analysis of the sum of its constituents. Can we think of $\mathrm{X}$ as a whole of correlated classes? Is an organism a cellular pile joined by an individual-specific?

Imagine an average domestic dog as an example. If I meet a dog in the street, I do not think of it as a whole of cells. Instead I think that this dog is an organism. Perhaps this is the dog called Pluto. Pluto is simultaneously an individual and a member of the canine species. The tirst identity level defines a biological individual, the second one a species. Let us stress the first level, that of the individual.

As 1 have already said, I do not think of a dog as a whole of independent cellular units, that is I do not say this is a set of $6,786,978,356$ cells named Pluto. In addition, I do not say "this is the cellular whole Pluto". Nor do I think that if one of Pluto's constituents vary, then his identity ends and is substituted by another, such as 'Pluto II'. Common sense reminds us that biological identity involves a margin of structural variation (qualitative or quantitative variation) in itself and in its constituents. This variability-in. stability of biological individuality is an intrinsic property of organic systems, and is implicitly contained in the concept of whole ${ }^{13}$. However, if we stress organic variability, how can we say that an organism such as $X$ has an identity that continues over time?

In order to solve the above problems we first have to clarify which procedure of classification can (or should) be used when determining an organic system's identity. So far, we have acknowledged two possible approaches to the identity problem. (1) We think of an identity as a static process, then an object remains identical to itself $\left(X=X^{14}\right)$ and (2) we think of an identity as a dynamic process. In biology, we can find countless reasons to accept the second view. Thinking of identity as a unity, means we are basing (and limiting) our analysis on the s-relation. However, if we use the concept of a whole we can witness a variation amongst an organism's constituents. This variation annuls the equational relationship $\mathrm{X}=\mathrm{X}$ (point 
1). The nature of an organic system's identity, to be a whole, causes the collapse of equational relations ${ }^{15}$ of sort $X=X$ (Williams 1973, 1980).

One might object that, while the variable nature of otganic systems induces us to avoid thinking of identity (intuitively) as a static process (one-to-one relation-Perring 1997), in the case of an object like a stone we might use equational metrics to define the identity of the above object. Nevertheless, physics also states that a stone has structural transformations based on quantum phenomena, even if the number of a stone's atoms does not vary. The problem is that the bijective property $\mathrm{X}=\mathrm{X}$ involves an absolute coincidence within an object's requirements. According to the one-to-one relation, we think that an object does not vary neither structurally (the number of its atoms) or qualitatively (its colour, form, etc.). This immobility connotes each level of its structural complexity. The stone's micro-level has a continuous structural stability. This margin of variation avoids the coincidence of a stone's atomic structures at different temporal points. A bijective metric is not used in inorganic systems because structural variation is a physical property of organic and inorganic matter. We can then use the $\mathrm{X}=\mathrm{X}$ relation only in an abstract or theoretical sense. We symbolise an object and utilise one-dimensional mechanisms of representation (ahistorical dimension). However, if we pragmatically aim at understanding the nature of an object's identity, we need to stress the physical features of the objects under investigation ${ }^{16}$. This analysis asserts that both organic and inorganic systems have continuous variations within themselves (the Second Law of thermodynamics says that the entropy of isolated systems increases over time). Then an object can vary over time, and yet still be the same object ${ }^{17}$.

Returning now to our original conceptualisation of identity, I stated that whether our subject is an anthill or a cell culture, it has some constitutive variations in its elements. In different periods object's constituents (atoms, molecules, cells and so on) will not be identical (remember the sand dune), but they will be remain similar to their prior selves. So a relation that links these elements does exist, while the identity of an object involves its constitutive and qualitative variation in both its elements and in its self. I claim that identity is a plastic process in which an unitary entity, at a certain time (unity), varies through time (whole) while persisting.

Let us underline the reason of my emphasis of identity plasticity. When we talk about identity we signify that: (1) we define something as an identity, according to the description of its requirements within a determined time 
period. (2) we can recognise it at different times because it maintains features, which will lead me to describe its own identity as a temporally continuous identity. Both of these dynamics point out two peculiarities of identity problem: (1) The definition of an identity at a certain time and, (2) continuity of identity. Using the language of Nozick (1981) we will talk in the first case about Transverse Identity (TI), and in the second case Longitudinal Identity (LI). TI thinks of X's identity as a unity, while LI sees it as a whole that continues through time. TI and LI are intrinsically correlated $^{18}$. This conclusion proposes a basic principle of the identity of living beings: organism organism $X$ is an identity at a certain time that continues through time even if its constituents qualitatively or quantitatively change. We thus need to individuate continuity relations in the existence of objects through time ${ }^{19}$.

\subsection{Unifying Variable and Heterogeneous Systems}

We have seen that we do not consider dogs to be the whole of numerous cells, but rather as biological individuals. On the other hand, if we think of cells in a test tube we do not think of them as a biological individual, but simply as cells. What is the difference between organisms and whole of cells? What minimum requirement is needed to be considered an individual? Also, in the case of the test tube cells we have a whole $(Z)$ composed by cellular constituents $F(z) . F(z)$ cells qualitatively and structurally vary (cells die and reproduce). What is more, they have a common relationship of space and time. Why do cells in a test tube fail to compose an individual? The first intuitive response lies in the fact that a dog is an integrated locus of biological complexity. These are systems organised in hierarchical correlated structures that have common functions, such as reproduction, and translation of genetic codes. $Z$ does not possess composite hierarchical organisation, and is only a set of cells. Nevertheless, cells in a test tube constitute a whole with a reproductive function and shared genetic information. Where is the difference?

In order to respond to this question, we need to emphasise the linking conditions that unify a whole of elements into a global entity, an organism. We need to point out the causal conditions that transform a composite entity into a unitary entity. In parallel, because organic systems have continuous qualitative and structural variations within their constituents (they are heterogeneous and variable entities), we need to investigate the continuity 
of identity of variable structures (whole-unity). Organic systems act as whole-units. I defend the thesis that:

An organism $(X)$ is a composition of the constituents as a whole $[\mathrm{F}(\mathrm{x})]$ at a determined time 1 l. $X$ 's identity will continue over time if its $F(x)$ requirements causally continue in $\mathrm{F}(\mathrm{y})$ 's at $\mathrm{t} 2$, and if no other requirements $F(z)$ exist at $t 2$ having not derived in a casual sense from $\mathrm{F}(\mathrm{x})$ at $\mathrm{t1}$

The $\mathrm{Z}$ (test tube cells) is a unity, because cells share a common spatiotemporal relation $\left[\mathrm{Z}=\sum \mathrm{F}(\mathrm{z})\right.$ at $\mathrm{tl}$-observation..]. Z's cells multiply themselves through time (as cells reproduce) and are causally related because each cell generates others. This relationship clusters cells in a causal sense. The causal correlatedness of Z's cells guarantees their inner cohesiveness. It is vital to emphasis this biological property of organic systems, because an organism has either mechanisms of cellular substitutions or mechanisms of differentiation in tissues. The heterogeneity of integrated biological systems makes it difficult to describe factors of causal correlatedness of a biological individual merely as a similarity condition within its constituents (differentiated cells). Therefore, we also need to stress the causal correlatedness condition that clusters an organism in its unity (and continuity). In the case of cells in a test tube, the causal property that unifies the cellular whole refers to two phenomena: (1) Similar (cellular) units compose the whole $F(z)$ and, (2) $F(z)$ is a set of clones mutually interconnected by a relation of causal correlatedness. I have said that $\mathrm{F}(\mathrm{z})$ 's constituents are similar because cells have the 'same' genome. This element links cells in terms of constitutive similarity. At the same time, a genome organises the meiotic process of cells' reproductions. The whole $Z$ has a constitutive r-relation that clusters its constituents in terms of maximum similarity and causal correlatedness. The causal correlatedness clustering the cellular whole is the fact that $Z$ 's cells are clones of one initial cell. The cells-in-a-tube are causally r-related because they have a ancestor, and they produce other cells via their reproduction capacity. Contemporary, cellular constituents have a precise factor that safeguards their internal similarity independently to their differentiation: they possess the same genetic endowment.

Cells compose a unitary entity when causal correlatedness and similarity conditions are respected. An organism, then, is a unity because r-related constituents compose it, and it is a whole because (via $r$ ) its identity 
continues over time independently to its qualitative-structural changes ${ }^{20}$. In biological individuals, the r-relation in primis consists of the fact that its constituents are clones of an egg cell (differentiated cells have the same DNA, and they are result of a reproductive chain), and cells have a common DNA. DNA causally clusters cells in a whole-unity. At the same time DNA regulates cellular functions. Can we conclude that DNA is the basic element of the existence of living beings as individuals?

This need to emphasise the causal interconnection is reflected in Ereshefsky's definition of individual: "An entity is an individual only if its being that entity requires some appropriate causal connection between its parts" (Ereshefsky, 1991:97). If causal mechanisms are so important, which difference allows us to distinguish the identity of an organism from the one of a cellular whole? Is a cellular whole an individual? At this moment we have no elements to say that Pluto is an individual and $Z$ is a cellular whole. Also $Z$ possess the causal factors of organic systems. Why is Pluto $(X)$ an identity and $Z$ is not? Something diversifying $Z$ to $X$ has to exist. Both are wholes-units, and have a system of integration in their structural complexity. Can we say that their difference is only a difference of degree of complexity? Is it a qualitative ${ }^{21}$ difference?

$Z$ is the composition of sets of cellular elements. $Z$ has an existence limited to the cellular r-relation. $X$ is an organised organic system that acts and behaves as a unitary entity. It is a heterogeneous system, hierarchically organised in a unitary (cohesive) entity. It is a whole of functional (composite) units organised in systems strictly correlated to form a unitary dimension. This interrelation is reinforced by the possibility of functionally interacting with environment, and by interconnection among organic systems, Take for example, the case of complex sensorimotor systems. The interaction between central nervous system, muscular systems, at every level of their integrated complexity, causes a motor act. Synergy connotes numerous biological phenomena, such as metabolism, neural events, and mechanisms of vision, and the endocrine system. An organism is an organised structure that has a purpose ('organon' in Greek means 'instrument'). Its aim is the maintenance of individual's existence.

An organism is a homogeneous structure of heterogeneous constituents that join in a variable and composite unity persisting over time despite its structural-qqualitative changes. The organism is a homeostatic system. It has the ability to maintain an internal biological harmony.

Here I am using the concept of homeostasis as advocated by Emest Nagel (1961). Just like a homeostatic system maintains a variable state in 
constant rates, in the same way an organism keeps its organisation constant depending on the variability of environment conditions of its habitat ${ }^{22}$. Using the words of William Harms (1997) we can say that organisms "maintain certain relationships between transient states of organism and transient states of the world". Homeostasis, then, involves the presence of internal relations that cluster the organism's biological constituents in subsets (organic composite units) whose sum form (but not explain) the global entity - a living being. The diversity of biological sets and their functions refers to the same whole-unity nature of organic systems. Homeostasis condenses this whole and its continuity. An organism thus is a unity-in-diversity, it is an organic unity.

\subsection{Organismic Biology}

The so-called organismic biologists advocated the organic unity concept during the 1940's. The zoologist Ritter (1919) has coined the term 'organismalism'. He used this concept to defend his thesis that "Organism in its reality is as essential to an explanation of its elements as elements are to an explanation of the organism" (Ritter 1919). Subsequent writers have changed this term to 'organismic'. The central point of organismic biology is that an organism is a highly organised system where its biological meaning (and the meaning of its activity) cannot be understood as the sum of the activity of the parts, of its biological constituents. This means that when we wonder about the meaning of a living being we cannot explain its existonce as a physicochemical phenomenon or attribute a contingent value to a singular organisms' constituent (for example, the brain). On the contrary, an organism is considered as a locus of integrated complexity, whose meaning refers to its composite nature. The members of this school all agreed upon this point, but they applied the organismic theory to different expressions of life. We can define two fundamental areas of application - behaviour and physiology. I will analyse these applications separately.

Russell (1938) underlined the notion that behavioural programs in organisms are the expression of a biological activity that is a result of physical matter. In the way we cannot explain the meaning of an organism by one (or the sum of the) requirement(s) of its complexity, in the same way we cannot understand organism's behaviours until we understand its functions and its aims. We cannot decompose the behaviours of organisms into a set of significant behavioural activities expressed in particular situations, because we have to absorb in our investigation the physiological, 
ethnological, and social systems concurring together to this directive. Russell's conclusion referred to a fundamental principle of organismic biology:

The laws determining the behaviour of a part in a determinate organisation level, are silent about behaviours of parts in higher organisation levels (Woodger, 1948).

This demands that we study the behaviours of living matter, either at its micro-complexity (cells, tissues, etc) or at the macro-complexity of animal behavioural programs, by analysing the relationship between relative systems at each level of organisation, and in their globularity. For instance if we want to study neurones' behaviours in the brain's neurotopography, we have to study the interrelations between neurones and neural maps without limiting our investigation to molecular relations between nerve cells ${ }^{23}$. Clearly this approach stressed the internal relations between structural complexity of living matter and its expression in the composite and correlated entity organism. The fundamental idea is that organisms have a whole of physical parts organised in hierarchically combined structures. We can define an organism as a cohabitation of a structural complexity that cannot be exhausted either in its physical meaning (we consider the phenomenon of life to be explained in terms of a set of physicochemical mechanisms), or in the sum of the elements forming it ${ }^{24}$. 

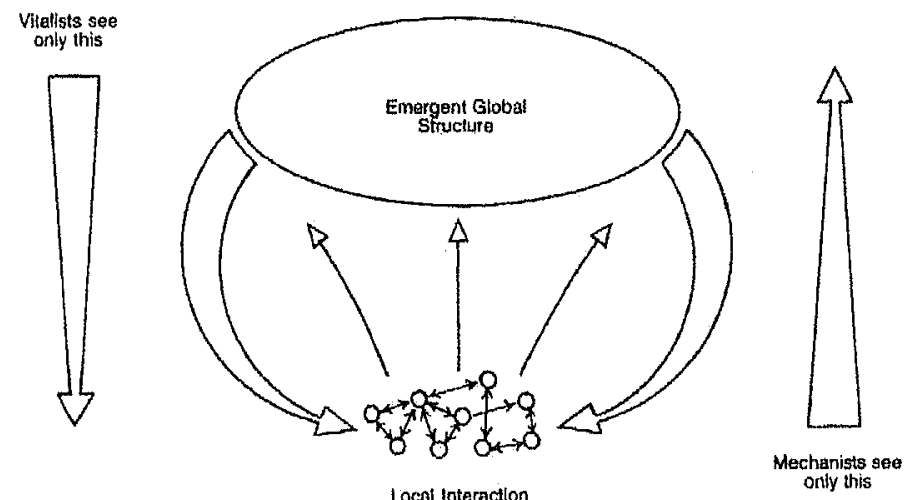

Mechanists see
orty this

Locai Interactlon

This ligure shows different ways to view the world. Vitalists will look for non-physical properties that determine the behaviour (or causes) of the observed object. Mechanists will look lor causal mechanisms. Emergent biology will explain the complexity of the observed object in itself. (Lewin 1992:189)

Organisms become a locus of functional and structural integration or a system of integration within biological characters. The coexistence of heterogeneous systems in a unitary entity introduces a basic problem of biology: the emergence of features that cannot be deduced from the knowledge of the organism's constituents.

Mayr defined emergence as follows: "When two entities are combined at a higher level of integration, not all the properties of the new entity are necessarily a logical or predictable consequence of the properties of the constituents" (Mayr 1988). According to him, the heterogeneous entity 'organism' cannot be considered as a sum of its biological constituents ${ }^{25}$ (because this heterogeneity is unified in a cohesive homogeneity that have individual specific properties), and synergetic relationships among organism's constituents unify an organism into a unitary entity that has properties and peculiarities of its own. This concept has been strongly criticised by some scientists because the emphasis on an organism's macrolevel does not clarify the relation between its micro- and macro-levels. 
However, it is important to emphasise that even if practical applications of organimal theory are criticised, nevertheless the organic unity concept ${ }^{26}$ is a standard concept in biology (Ayala 1973; Stebbins 1969). Proponents of the so-labelled autopoiesis theory (Varela 1979, 1991, Maturana and Varela 1980) have advocated it. The central idea of autopoiesis theory is that an organism is a self-determined complex dimension that maintains an individual (self-determined) integrity over time 27

The autopoiesis view, then, advocates that the 'sameness' of an organism becomes the basic feature of an individual's life. Clearly advocates of autopoiesis do not think of the individuality in reductionist terms. They defend a thesis that is similar to the one of organismic biology: "an organism is a variable whole' and underlines a logic of continuity in the organism's life over time. Given this, I do not see remarkable differences between organismic biology and autopoiesis theory. The autopoiesis theory links organismic views to the temporal persistence of organisms. If organismic biology stressed the identity of organism at certain times, autopoiesis theories addressed the same problems through time. Clearly, my view considers identity to be a process that is substantially concurrent with autopoiesis ideas. However I do not agree with the autopoiesis idea that an autopoietic system has a biological autonomy from the environmental influences - that the autopoietic system defines itself in contrast with its environment (Meijsing 1997:55, Varela 1991:85). This is because the selfreferential capacities of organisms do not exclude them from their environment, they simply allow the maintenance of an individual's existence depending on envirommental factors. This is true both for mental phenomena (informational closure) and a number of organisms' capacity to adapt to its environment ${ }^{28}$ (from immune system, endocrine system, and the thermal regulatory systems). I will come back to the relationship between organism-environment later on (paragraphs $2.4,2.5,2.6$ ). Let us focus here on the concept of biological individuality.

I accept the concept of organisms that has been put forward by certain proponents of autopoiesis, but I reject the absoluteness of the individual's existence as a completely autonomous entity. On the contrary I defend the thesis that an organism's existence is (also) a process of dialogue with its environment.

We could say that organismic biology with its emphasis on the unitary nature of living beings (and emergence problems) individuated a central problem of modern biology. In the natural sciences we have to approach the 
individual problem by stressing the unitary nature of organisms and we cannot focus biological analysis on an organism's certain features without emphasising the interrelations among the organism's constituents (Holland 1998). One of the harsher criticisms against organismic biology involves its defence of an explanatory methodology that refutes the reduction of biological phenomena to simple physicochemical mechanisms. This idea weakens the heuristic value of microbiology and the causality and prediction in biology (Beckner 1959). Beckner, talking about the "emergence of new qualities at higher levels of integration", does not mean that an analysis of biological properties at lower levels of integration (for example molecules) looses its heuristic validity. Simply he means that if we pass from the micro-level of living matter to the macro-level of biological integration we must consider the whole set of interacting causes. Because of this phenomenon the possibility of its prediction and/or explanation is reduced. The cohabitation of interacting causes in biological phenomena is a property of living matter in its different manifestations (from genetics to physiology, from medicine to psychology).

Another criticism of the emergence theory has come from reductionist philosophers. Hempel (1953:335-336) underlined the epistemological and methodological dependence of emergent properties in biology. He spoke of relative emergence in the sense that "a certain property which seems emergent in terms of a certain theory may not be emergent with respect to different theoretical contexts" (Egidi 1987:158). Feigl (1974:520-559) stressed the epistemic nature of emergence, Popper (1982:131-162) its logical nature. These philosophers have approached the emergence problem as a logical question. Clearly, the complex structure of biological theories can be inferred to advocate the emergence of new 'emergent' puzzles that cannot be explained or reduced to the already known elements. For example, when we wonder 'why' some living beings had developed the capacity to fly, rather than studying which physiological changes had lead to this phenomenon, we deal with emerging puzzles wondering why natural evolution has allowed the survival of flying creatures (from insects to birds). This means those emergent dilemmas are inherent to explanations considered as a hermeneutic approach to reality.

Analogies or associations among different implications of the observed phenomenon create emergent puzzles, yet this is a remark that we can extend to all the explanatory systems based on comparative analyses. The problem of emergence is not simply a logical question, but an empirical one. It refers to the approach of biological phenomena at their macro-level. 
When we deal with a phenomenon in its macro complexity, and we explore living beings as complex entities whose features should be explained by looking at living beings as 'wholes' rather than cell-machines (molecular biology), we necessarily deal with 'emergent' questions. This does not mean to deny the need to explore physical chemical features of living beings. It simply means that the value attributed to these elements is not exhaustive. When we think of living beings as organic units we simply look at them in a different way than mechanistics 29 .

According to this, in the following pages I will claim that organismic theory can be a useful view to explore the limits of mechanistic theory and I will stress those conceptual factors that encourage me to say that organisms are organic unity. I will analyse different approaches used to explain an organism's individuality in modern philosophy and biology. This study aims at proposing a new use for organismic biology within the philosophy of biology.

\subsection{Organisms as Organic Units}

In the previous paragraphs 1 said that an organic unity was a heterogeneous and composite entity having a unitary value on its own (Nozick, 1981:464-476). Let us apply this concept to living beings. We can classify an organism's biological constituents in sets (functional systems $\Rightarrow$ organs $\Rightarrow$ tissues $\Rightarrow$ cells $\Rightarrow$ proteins $\Rightarrow$ genes). Every class has similarities, and partial dissimilarities with others. An internal relationship exists which link the classes. The causal relationship among organism's constituents (at any level of complexity of its hierarchical structures) refers to the biological property: entities at one level are computed into new entities at the next higher level (Mayr 1981:14-15). The united 'organism' is the result of a unity/diversity of biological constituents, it is an organic unity (Agar 1951; Lillie 1950).

A standard example of organic unity is a picture. If we think of a picture, our perception shows us a polychromatic system as a unity. In reality each colour is single, but colours all together are something else, they form a polychromatic structure that has a unitary nature. The picture is an organic unity. The unity of a picture is not limited to the set of its polychromatic elements, and in addition they form the picture. This property belongs also to living beings. Constituent sub-units (cells, tissues, and organs) form the organism. It is the shared space that unifies the requirements together. At 
the same time, it is a unitary entity because coexistence of its heterogeneous and integrated constituents defines its identity on its own, and its continuity, independently of its composite and variable nature. The identity is a locus of diversities integrated in a unitary entity. It is not limited to some particular requirements of an organism but it is a unitary dimension. The features of organic systems can be defined in their nature of wholes (homogeneous entity) and in the impossibility to deduce their peculiarities in the knowledge of one (or the sum of) level(s) of the biological complexity of their own constituents. The unitary meaning of unified complexity of a biological system leads us to be aware of the emergent properties of the whole in its complexity ${ }^{30}$.

As I have already said, an organism is a unity because it has a spatiotemporal relation among its constituents. It is a whole because its identity continues via the similarity and causality conditions. It is a wholeunity because it is an unilary entity that maintains an inner variability in its continuity. And it is an organic unity because we cannot explain its integrated individuality as the sum of its constituents, and because it has internal processes of self-cohesiveness. These principles refer to the concept of an organism as a homeostatic system. The homeostatic nature of an organism maintains its inner unity toward the variability of extemal conditions. It ensures the continuity of organism (its plastic stability) in the variability of Life. The clustering properties among the organism's constituents (biological integration) thus become basic elements to be taken into account in dealing with the organisms' identity problem (at a certain time and trough time). Since these factors are numerous (from metabolism to the immune system, from neurotopography to mental states, and so on), it follows that the r-relation is a set of factors possessing a common aim, unifying the unity-in-complexity of an 'organism'. Biological individuality becomes a variable process organised by a logic - to join organisms' variability in an entity that persists while varying. The clustering r-factors ensure an organism's continuity and safeguards the biological individuality via homeostatic processes. The constituents of an organism regenerate and realise the system as a whole ${ }^{31}$, they define a unity by their organisation, and constitute an individuality because they possess an identity by keeping their organisation constant.

When we pass from the macro-level of complexity of an organism (its phenotype) to its micro-level of complexity, we can individuate the causal relations linking its constituents. At a molecular level, DNA is the causal link among an organism's constituents. But it is only one of the clustering 
factors that unifies the individual. As organismic biology stated, living beings are systems of integrated complexity. Their unifying factors are subservient of the maintenance of organism's own organisation. This neglects the role of specific biological constituents of organisms ${ }^{32}$. The organic unity of the 'organism' is a unitary entity in its heterogeneity. We cannot attribute to some of the organism's constituents a central function in its existence, just as we cannot see of a picture's identity as a particular chromatic system.

We can synthesise the organic unity view as follows (Features of the Organic Unity 'Organism'):

\begin{tabular}{|c|c|c|}
\hline & Structural features & Qualitative fentures \\
\hline $\begin{array}{l}\text { Inner } \\
\text { Causality }\end{array}$ & $\begin{array}{l}\text { Cells are clones of a unique } \\
\text { cellular line. Every level of } \\
\text { the organism's integrated } \\
\text { complexity is causally linked } \\
\text { to the other. Organism's } \\
\text { constituents } \\
\text { interconnected. }\end{array}$ & $\begin{array}{l}\text { In an "organism' each level of } \\
\text { complexity is compounded into new } \\
\text { entities at the next ligher level of } \\
\text { complexity. The coexistence of systems } \\
\text { and their cohesive integration causes the } \\
\text { emergence of characters which cannot } \\
\text { be deduced by the knowledge of } \\
\text { organism's constituents. }\end{array}$ \\
\hline $\begin{array}{l}\text { Inner } \\
\text { Similarity }\end{array}$ & $\begin{array}{l}\text { Cells have DNA in common } \\
\text { and display a structural } \\
\text { (variable) similarity. }\end{array}$ & $\begin{array}{l}\text { Organisms' constituents have similar } \\
\text { physiological mechanisms producing } \\
\text { different results. }\end{array}$ \\
\hline $\begin{array}{l}\text { Homeostat } \\
\text { ic Function }\end{array}$ & $\begin{array}{l}\text { The organism's life is the } \\
\text { result of a structural } \\
\text { interconnection within } \\
\text { different biological systems. }\end{array}$ & $\begin{array}{l}\text { The organism's life is based on its } \\
\text { homeostatic structure and adapts its } \\
\text { inner-variability-in-the stability to } \\
\text { environmental pressures. }\end{array}$ \\
\hline $\begin{array}{l}\text { Unitary } \\
\text { Entity }\end{array}$ & $\begin{array}{l}\text { The organism is a cohesive } \\
\text { dimension of heterogeneous } \\
\text { constituents, sharing a space } \\
\text { time system and forming a } \\
\text { unitary locus of structural } \\
\text { integrated complexity. } \\
\text { Organisms behave as units. }\end{array}$ & $\begin{array}{l}\text { The organism is a unitary homeostatic } \\
\text { entity, which links itself to environment } \\
\text { and continues over time. }\end{array}$ \\
\hline
\end{tabular}

\subsection{Why isn't a Cellular Culture an Independent Identity?}

Now we have some elements to know what an organism is, although I have not yet explained how contingent properties make living beings into individuals. The open question is 'Why cells-in-a-test-tube $(\mathrm{Z})$ 
do not form a biological individual?' Now we can solve this problem. We have seen that organic systems have a specific spatiotemporal relation joining their own constituents. However, this spatiotemporal tightness must be reinforced by a "degree of cohesiveness and integration with respect to the causal forces impinging upon them" (Horvart 1997:227). This involves that the spatial and temporal links between the individual's constituents, while being a necessary clustering factor, cannot be considered as a sufficient condition to define an organism as an individual entity. They individuate a cohesive dimension among numerous biological entities (such as cells), but they cannot explain the unitary nature of individuals. As Liden and Oxelman (1989) point out, the autonomous and individual nature of living beings refers to their capacity to react (behave) to external factors. This means that, in order to be individuals, organisms need to react as unitary entities at different levels of their biological complexity, from the molecular level to phenotypic levels. This capacity differentiates individuals from cell-wholes. The active interaction among an organism's constituents explains its individuality and its capacity to behave as a unit ${ }^{33}$.

A cell culture does not have this capacity to behave as a unit, and to react to external factors (such as environmental factors, or epidemic factors). An organism is an organic unity whose degree of biological complexity refers to its biological complexity and its capacity to behave (react) as a unity. When this last capacity is absent, we cannot think of organic systems as individuals ${ }^{34}$. In parallel, $\mathrm{Z}$ is not an identity because it is not an organic unity. Its existence can be considered as a whole-unity, but the r-relation linking its cells is unique. Its identity does not have a sufficient degree of constitutive dissimilarities to constitute an organic unity. In other words, cells-in-a-test-tube are r-related, but do not form a composite and heterogeneous unitary entity. They do not form an organism - they constitute simply of a cellular whole-unity that displays a degree of diversification among its constituents insufficient to define Z's identity as a unity-in-diversity. It seems that a border of existence exists (not classifiable) by which organism's identity constitutes itself as a heterogeneity which is joined into a (variable and plastic) unitary dimension that dialogues with environment. Is the organic unity view thinking of a colloquial relation organism-environment as the minimal condition to define an organism an individual? If DNA has a causal role in living beings' existence, is the genome the central element of biological individuals? 


\section{NOTES}

I In this disscrtation I will approach the problem of individuality. I will explore personal identity problems in order to find a coherent and justified explanation of the meaning of an individual's existence. Clearly, this approach will be interdisciplinary. Biology, neuroscience, genetics, and philosophy will be cqually used to explain identity puzzles. Obviously, this apprnach does not atempt to defend an exhaustive theory of the above problems. It simply aims to defend a view that reasonably explains identity dilemma through a Socratic approach.

'When I use the word Personal-ldentity I signify the biological-psychological features of an individual at a determinate time and throughout time.

${ }^{3}$ Walking down the beach, I may see a red stone, a small stone, a stone that has spent its life in the wash of the tide. All are stones, yet none are the same. Unlike this author, a stone can never linguistically claim its identity. The identity of an object (such as a stonc) that is deprived of reflexive self-reference refers to an observer's outside reference and personal classificationcriterion. It is my observation that separates this single stone from its ancestry

${ }^{4}$ In this sense the identity concept needs the existence of an outside reference (or a reference system) to attribute the identity valve to the experienced object.

'We have perceptual inputs of the stone's existence at its macro level, and we think of that stone as an integrated system possessing a unitary meaning. We can talk of identity simultaneously as (1) the outside identity and, (2) the identity from its inside. Self-aware organisms have both of these identity-features: they are aware of their individual existence, and they are identities for their observers. This involves the realisation of a thing's existence to their observers induces me to analyse the identity-criteria of classification.

- Moreover this condition does not completely explain the identity of composite and heterogeneous objects such as organisms (composed by cells, organs, tissues, etc.). We cannol limit the concept of unity to the maximum similarity condition, because this similarity could be absent, or the description of the object could not individuate its unitary nature. We need to look for further elements that causally join the object. Let us consider an example: a car. It is composed of difterent materials, systems, and parts. The heterogeneity of its elements does not obstruct its unitary nature. If the concept of unity were limited to the condition of similarity, we would bave concluded that the car is not a unity. We think of it as a unitary (composite) entity because our criterion of classification attributes to the spatio-temporal relation that joins the car's constituents a cohesive property. In this sense, car is a unity independent to the heterogeneity of its constituents.

' $\mathrm{A}$ analysis of this problem is in Nozick 1981:118-134. The American philosopher pondered on the Theseus Ship (TS) identity. The planks of the ship had changed over time. Does its identity continue over time despite its constitutive changes? Nozick thought that if a causal relationship amongst TS' constituents exists, its identity will continue through time. This means that an identity will absorb within itself new constituents if a causal relation within its constituents continues over time. Organ-transplants are an interesting example. See Nozick 1981:33-34

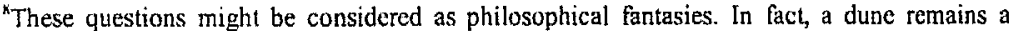
determinate entity while varying. Nevertheless, in clarifying reasons that allow us to say its existence conlinues over time, we can understand the nature of variable and composite identities, such as living beings. Reflections on the send-dune's identity thus allow us to clarify the process of organisms' persistence over time as a determinate individuals. 


\begin{abstract}
${ }^{9}$ With the term 'sub-units' I mean the constituents of a complex entity. For example, sub-units of an embryo are embryonic cells, tissues as well as their constituents (DNA, RNA, etc.).

111 "Emergentism is a label for theories which assert the presence, in the realm of living beings and organic lifc, of elements which are not continuous with what went before and which are therefore 'emergent', or irreducible to the already known elements". Egidi 1987, in Radnitzky 1987:157

18 "Systems at each hierarchical level have two properties. They act as wholes (as though they were homogeneous entity), and their characteristic cannot be deduced (even in theory) fiom the most complete knowledge of the constituents. Such emergence of new properties occurs also throughout the inanimate world, but only organisms show such dramatic emergence of new characteristics at every hierarchical level of the system" (Mayr 1988:15).

${ }^{12}$ Given that individuals are composite and variable entities, we have to approach an identity problem (in biology) by emphasising the synergetic nature of biological phenomena, such as the relation between environment and organism, and mental states (in organisms having a sufficient development of their neural topographies).

${ }^{13}$ If we thought that the identity of a stone $X$ could be explained by the biunivocal dynamic $X=X$, then $X$ at $t 1$ should be identical to $X$ at 12 . If $X$ at $t 1=X$ at 12 were trie, the stone's atomic constituents did not (quantitatively, qualitatively, spatially) vary. But this it not true because, at a quantum level, the spatial structure of $X$ ' elements changes. The s-relation within $X$ ' sub- constituents remains the same 'even if is not identical to itself'. Atoms maintain a determinate spatintemporal relation even if their spatial structure is modificd. This involves that we have to consider identity a similerity-process within a thing's constituents and not a relation of the sort $\mathrm{X}=\mathrm{X}$. When we think of things' continuity we have to substitute the equational concept with the similarity concept.

14 Whit the term ' $X=X$ relation' 1 mean that an object is seen as perfectly identical to itself through time. Then I am using this expression thinking of mathematical identities rather than formal logic.

15. We can analytically approach this conclusion. If we define an object $X$ (at 11 ) by a variable factor $\mathrm{Fx}(\mathrm{y})$ that contains its requirements, it follows that $\mathrm{X}=\mathrm{Fx}(\mathrm{y})$. At a later time I can accept that equational metric $X=X$ may be the right dynamics of $X$ 's continuity, but only if I find perfect coincidence between $X$ 's description at $t 1-12$. Sinec $X$ at $11=F x(y)$, and $X 2$ at $t 2=$ F $x 2(y 2)$, for the transitive property of mathematical identities it follows that $F x(y)$ has to be identical to Fx2(y2). This is contrary to the initial premise stating; at different times $(11,12)$ the stnuclure of a whole varies. If we think of an organism's (cells' substitutions), or other examples of variable entitics (such as a sand dune), we sce that these identities have qualitative and constitutive changes. Knowing this, we arrive at the conclusion that: we cannot consider an object something that remains identical to itself. The relationship Fx $(y) \neq F \times 2(y 2)$, breaks the continuity of X's identity. We cannot, in this case, analyse identity problems through the equational dynamic $\mathrm{X}=\mathrm{X}$.

${ }^{16}$ Another possibility of employing $X=X$ relations in identity puzzles is to consider $X$ in a dualistic sense. In this case, the being $X$ (in a metaphysical or ontological sense) rests identically to itself because its existence refers to an eidetic property that is (pcr se) deprived of any direct link with physical matter. This elaim has metaphysical premises. In following this view, we can conclude that the identity of a stone is contained in a disembodied element, a further element which does not vary over time. If this hypothesis does not have rational and scientilic evidence, then it cannot be of significant use in our analysis.
\end{abstract}


${ }^{17}$ Another reason to accept views that consider identities to be dynamic processes refers to the classification problem. If we describe a stone $(X)$ at $t 1$ by complex physical descriptions (atom relations of state for example), we need to describe the structure of its atomic constituents. But Heisenberg's indetermination principle states that our observation modities and interacts with structure of the observed object. (Heisenberg (1958) stated that each observation and measurement of phenomena influences the state of the systems being analysed, thus erasing possibilities of objectivity.) This means, as X's description (at $t 1$ ) is blemished by our observation, the observation at a later time $t 2$ will likewise be perturbed. We thus have noncoincidental descriptions of $X$ at different times. If these descriptions are not congruent, then we can only conclude that $X$ at $t 1$ is not equal to $X$ at $t 2$. $\Lambda$ gain, this conclusion is contraty to
the equational dynamic $X=X$.

is "I see no compelling reason, however, why existence must be conceived of in these stages, first entity at a time (transverse identity) followed by entity over time (longitudinal entity). Indeed, if causal interconnections among parts are part of closeness in the transverse metric space then, via causal connection, time already enters into transverse identity and so is not restricted only to longitudinal considerations. We can imagine the abstract metrie space to include time as one of its dimensions; existence, the classifying which produces entities, takes place in one dell swoop, rather than in the stages of transverse followed by longitudinal. On this view, the distinction between transverse and longitudinal identity is derivative, abstracted from a clumping together in a large space". (Noxick, 1981:86-87)

${ }^{19}$ The continuity problem in a whole's identity refers to two logical dynamics: the proximity and similarity coneepts. Take the case of an object ( $X$, for the sake of simplicity) at $t l$. When we wonder if X's identity at $t l$ continues in another object $(Y)$ at a later time $t 2$, we have to be aware that $X$ and $Y$ are not congruent. If these objects are not identical, $Y$ would be the most similar object (the closest continuer) to $X$. This means that $Y$ will be the object that has the maximum degrec of similarity (strictest continuity) with $X$ at any level of its integrated complexity. If $X$ 's constituents are $F(x)$, and $Y^{\prime}$ 's constituents are $F(y)$, it follows that $X$ continues in $Y$ if $F(x)$ continues in $F(y)$. This indicates that the degree of proximity between $X$ at 11 and $Y$ at 12 defines $X$ 's continuity in $Y$. Congruently, the degres of similarity between $F(x)$ at $t 1$ and $F(y)$ at the later time $t 2$, connotes $Y$ as the closest continuer of $X$ even if both have some (qualitative-structural) differences $\left[F(x) \neq I^{r}(y)\right]$.

${ }^{20}$ The sand dune is a Whole-Unity. A Unity because its components are spatially joined to others (if grains are not in a same place we have no dune); a whole because its grains ehange continuously.

${ }^{21}$ Goodwin emphasised the qualitative features of an organism as follows: "... by saying that there is some kind of organisation that is distinctive to the living thing we canl move to a closer appreciation of the quality of the organism. (...) l'm talking about the organism as the cause and the effect of itself, its intrinsic order and organisation. Genes don't cause organisms. There are ${ }_{22}$ causes of organism. Organisms are self-causing agencies" (Goodwin in Lewin 1992:180)

${ }^{22}$ Cannon introduced this idea in 1929) (Cannon 1968). He said that organisms had a general propensity to maintain "relatively steady internal state in spite of changing external conditions" (Wiepkema 1997:95, Toates 1986). Common cxamples arc the maintenance of body temperature, the glucose level of the blood, etc..

${ }^{23}$ Let us think of the application of quantum physies to brain's activities in modern neurophysics: Penrose 1991; Lochwood 1089; Zohar 1990

"Using the words of lirnest Nagel (1951:327-338) we can say that: " the behaviour $B$ of a system $S$ is more the sum of behaviour bl, .... bn of its parts $s 1, \ldots$ sn with respect to an 
antecedently specified theory $\mathrm{T}$, if (1) B is an instance of a law $\mathrm{L}$; (2) $\mathrm{L}$ is not a part of T; (3) the laws in $T$ describe $s 1, s 2, \ldots$. sn in such a way they explain $b 1, b 2 \ldots . . b n$, and (4) $L$ is not deslucible from a description of $\$ 1, s 2, \ldots$ sn together with laws in $T$ ' (quoted in Morton 1972:549-551)

${ }^{25}$ "Emergence is ubiquitous in nature. It is what turns the simple interactions between pairs of ants into complex colony-wide behaviours, changing the colony into what appears to be a single 'superorganism'. And it is the process that allows the human immune system to create a seemingly endless varicty of antibodies from a small number of genes. In every case, the whole is so much more than the sum of the parts". (Regine 1998:39-42)

${ }_{26}$ This concept is also present in Psychology (Gestalt psychology): Arnheim 1974, Gombrich 1979. The concept of the complexity of behavioural programs is a central point in ethology, and the synergy of interacting factors in the causation of a phenomenon is a central paradigm in evolutionary biology.

27 " Autopoiesis attempts to capture the mechanism that generates the identity of living beings. This identity ean be loosely described as self-reproduced coherence: the autopoietic mechanism will maintain itself as a distinct unity as long its basic concatenation of processes is kept intact in the face of perturbations, and will disappear when confronted with a too drastic perturbation" (Stewart 1992:17)

${ }_{28}$ I am aware that the difierence between autopoiesis and organismal theory should be further analysed. However, because of the economy of this thesis, I cannot adequately focus on this issue. Those who want to read issues related the autopoiesis theory can see: Cerruti E. (1999) La Danza che crea, Milano Il Saggiatore. A paper that criticises the concept of autopoiesis by stressing its solipsistic nature is Lelas Srdjan, 1986 (Lelas, Srdjan, 1986. Epistemic implications of two biological concepts. Philosophica 37: 127-150).

${ }^{29}$ Another criticism against organismic biology was that this theory defended a set of truisms: No part of a bird can fy, an orgamic part, such as a cell, has properties in the organism that it does not have in isolation from the organism, all these formulas have been considered tnisms. I only wish to present a general objection to this criticism. The togical meaning of an idea is important when its extension opens the possibility to "approach" a phenomenon in a different way. When we realise that these truisms introduced concepts that constituted primary conceptual schemes in various biological seiences, such as ethology, microbiology, and evolution theory, this criticism becomes nonsensical. The Organic unity concept is simply a new approach to explain or study the complexity of a phenomenon.

${ }^{30}$ Biologists used the word 'emergence' to indicate the manifestation of new properties of biological systems in their global complexity. Biology has to measure its explanatory activity with the emergence of new properties of complex systems at every level of the hierarchal system. This peculiarily of living matter makes recognition of downward causation difficult (Campbell, 1974:163-177), or the research done in isolated systems diflicult (eg tests tubes).

${ }^{31}$ Biologists called these systems autopoiesis systems (Marutana and Varela, 1973; and Varela 1991). I do nol think that a substantial difference between organic unity concept and autopoiesis exists. The autopoiesis view (Marutana and Varela, 1973; 1991:79-107) underlinco the autonomy of living beings with regard to thcir environment. I do not agree with this conclusion. The relationship organism-environment will be explored in the pages of this chapter.

${ }^{32}$ On this topic see: Wagner 1994:282-283; Cotman 1981:684-784, 1981; Haken 1978 
${ }^{33}$ On the individual's responses to causal forces impinging living beings see: Mishler and Brandon 1987, Mishler and Donouge 1982. The interactions between genes has been analysed by Kaufmann, 1993

${ }^{34}$ Ciearly those organisms that have sufficiently developed their central nervous systems will reinforce their biological self-awareness -the capacity to react as a unity- with mental traits. Their degree of organic unity unification will be higher from the one of pre-conscious -or unconscious- organisms. 


\section{I.2 Genetic Identity}

The theory of organic unity attributes to DNA a primary role in organisms' existence as individuals. Nevertheless, in order to clarify the role of genetic identity for the existence of living beings, first we have to deal with a specific biological theory that considers DNA the basic element of Life. Mechanists think of DNA as the principle by which we can understand mechanisms-properties of living systems. In this chapter 1 will claim the impossibility of completely explaining the features of organic systems via DNA. This analysis aims at stressing the role DNA plays in the cohesiveness between organism's constituents, while arguing for the impossibility of explaining natural phenomena through genetics alone.

\subsection{Biological Determinism: The Theory-of-the-Farm (TF)}

Many molecular biologists have advocated reductionism citing primary principles on which biological individuality is based. There are numerous examples of this paradigm including Monod (1967), Eigen (1975) and Watson (1998). The common idea uniting these scientists is the possibility of isolating basic elements with which to explain an organic system's feature - the genome. The conceptual basis of this common point of view is that biological phenomena are expressions of chemical-physical processes. This posits that the genotype of a cell determines its manifest phenotypic appearance. Within this framework, physical properties of living matter become the basic element of their biological existence.

For example, Monod underlined the causal value of DNA in an organism's biological existence (DNA contains 'all that is important' for the life of an organism). Eigen considered organisms' biological features as the result of genetic peculiarities. He reduced natural evolution to mechanical (stochastic) processes of population genetics and advocated that adaptive processes referred to the affirmation, at a species level, of adaptive gene pools ('quasi-species' model). Dawkins focused his research on the phenotype as a 'container' of genes, which themselves are considered the causal principle of Life. All these theories propose a specific paradigmatic view: DNA is the 'basic' principle of Life.

I will approach such a mechanistic biology via the theory of one of its promoters, J. Monod. In Chance and Necessity (1967), the French molecular biologist emphasised two biological phenomena: invariance ${ }^{1}$ and 
teleonomy. He based his work on the physicochemical features of DNA. Monod conceived of the causal role of DNA in biological phenomena as the physicochemical mechanisms of protein production under their physical laws: emphasising its irreversibility ${ }^{2}$. DNA contains chemical information to synthesise proteins, and these can be modified and inherited in descendants only via DNA. According to Monod, DNA became the crucial dimension containing all significant biological information - it has a central role in biological phenomena ${ }^{3}$.

Monod is a good example of a mechanistic biologist. In his view, organisms are considered as Gene Farms ("cells-as-factory", Lewontin 1995'). They are the result of genes' expression aiming at producing - and transmitting - other genes. I call this view Theory-of-the-Farm (TF). We can summarise TF's view as follows: Since proteins determine organisms, and these proteins are specified by particular nucleic sequences; DNA is the basic element of biological individuality. According to TF, I can describe an organism by the following parameters: 1) I describe X's genetic structure; 2) I suppose that genes will be mechanistically expressed in an organism's genotype and (3) I can deduce the features of that organism (at a certain time, and through time) via the analysis of its genome. May we assume that biological identity is explained by a set of numerical and chemical data? Is it sufficient to know this chemical information to define and understand the biological meaning of individuals?

Advocates of TF say that when we assume that genetic identity contains all the useful information of an individuals' existence, we think of the genome as a coherent structure organising an organism's biological function through physicochemical laws ${ }^{5}$. This structure cannot vary if 1 am to talk of an individual's genetic identity. Let us consider this problem an open question. In the following pages I will show that DNA is an ambiguous and dynamic structure that changes over time.

First I shall stress the philosophical and biological implications of TF. The basic conceptual problem does not lie in the possibility of exhaustively describing DNA structure, but in the meaning that this element has toward biological phenomena. If we accept TF, we have to consider DNA as the organisms' causal principle at every level of their integrated biological complexity. In doing this, we attribute to DNA numerous properties: 
1) DNA is the basic (causal) element of $X$ 's identity;

2) DNA is a non-contradictory biological element, since its function can be explained by one-directional physicochemical mechanisms;

3) DNA regulates and causes phenotypic existence, which in turn cannot influence the DNA structure.

According to TF, the phenotypic expression of genes is merely the actuation of physicochemical phenomena. The phenotype is the mechanistic expression of chemical data contained in DNA. DNA converses with the phenotype only by translating its information. It controls the rules of communication and, at the same time, it provides the topic of conversation. It is not a dialogue, but a monologue. The knowledge of genetic information will clarify the phenomenon of Life. In an extensive sense, when we know the complete genetic sequence of a species, and the rules of their phenotypic translation, we will possess the knowledge to explain individual and species-specific biological features ${ }^{6}$.

\subsection{The Paradox of Monozygotic Twins}

I will now present an example that is problematic for the TF view: mono-zygotic twins. In human pregnancy if the fertilised egg (embryo) splits after four days, a particular type of identical twin pregnancy occurs known as monochorionic or monozygotic twins. This duplication generates two distinct individuals whose genome is identical (Dulbecco 1995). They possess an identical genetic endowment. Two problematic implications arise:

1) From a mechanistic perspective, two identical genetic identities should be expressed as two identical organisms. Both individuals should be phenotypically identical;

2) If we think of an individual's genetic uniqueness, how can we accept the existence of numerous identical genetic endowments (i.e. clones)?

The first paradox of monozygotic twins is the presence of two identical genetic structures. If $X$ is one twin and $Y$ the other, we ought to conclude that $\mathrm{X}=\mathrm{Y}$. And this contradicts the genetic identity concept where organisms have a (structural) genetic uniqueness. If the Theory-of-the-Farn is true, the case of monozygotic twins is a paradox. Since $X=X$, and at the same time $X=Y$, it follows that $X$ is identical to itself and to another organism (its 
brother). We have to suppose the existence of particular cases causing the collapse of genetic uniqueness concept. Following this view the individual $X$ does not exist, the set of $C(x)$ individuals exist - twins can be an indefinite number $n$, or we can clone $X$. This conclusion destroys the identity concept as singularity ${ }^{7}$. How can I accept that organisms can simultaneously have different identities and be the same organism? Do we have to completely reformulate the identity concept?

Let us consider the second problem. If we attribute to the genotype a basic value in an individual-problem, it follows that $\mathrm{X}$ and $\mathrm{Y}$ have 'qualitatively and structurally' identical genotypes. If we agree with the TF view that only one translation of DNA exists, we arrive at the paradoxical conclusion that since $\mathrm{X}$ and $\mathrm{Y}$ have the same genotype, they will both have the same phenotype as the expression of this shared genetic code. If phenotypic expression of their genome is mechanistically determined, we ought to conclude that twins are perfectly coincident. But this conclusion is unacceptable, because organisms have a 'qualitative-structural' biological uniqueness that is evidenced by their neurological and physiological peculiarities (expressed in their neuronal anatomy, homeostatic functions, immune system, etc. -Wright 1997). This phenotypic difference clashes with the view that upholds the one-directionality of phenotypic expression of genes $^{8}$.

"Monozygotic twins should not be described as "identical' because there may be mayor discordance within a pair not only in birth weight and personality but in congenital anomalies and even genctic disease." (Bryan 1998:51)

A clear example implying that it is impossible to identify organisms' features through their genome alone is the expression of Hox genes. Hox genes play a central role in the body axis of vertebrates: they contain information on the morphological differentiation of the corporeal regions at a specific axial level (Hunt and Krumlauf 1992:227-256; McGinnins and Krumlauf 1992:283-302). Biologists focus their research upon linking homologous body regions to a specific Hox code. But the comparative analysis of Hox code expressions in axial differentiation, stated that the gene activation cascade does not produce an identical expression of Hox genes. The genetic identity does not necessarily coincide with the phenetic expression of gene sequences. (Wagner 1996:11, used this example to define the independence of the "evolution of genes and developmental patterning mechanisms from character evolution".) 
The view that proposes mechanistic expression of genes in the phenotype is ambiguous. On the one hand, the causal role of DNA in biological phenomena (in protein and enzyme production, or in reproductive phenomena) is unquestionable. On the other hand, the synergetic properties of biological phenomena (for example: gene expression, embryonic development, neurotopography, mitotic and meiotic mechanisms or metabolism), de-emphasise the causal role of DNA in biology. In this sense, the emphasis on genetic endowment as the basic causal element of an organism's life is confuted by experimental evidence at all levels of integrated complexity that form the heterogeneous and cohesive unity of the 'organism'. In realising that TF cannot solve the monozygotic twins paradox, we must look for a theory able to avoid this paradox and to explain an organism's stability over time.

\section{3. 'Talkative DNA' Theory}

Below I shall consider the view that sees DNA as a plastic dimension. I will present data supporting this view, and I shall link these biological elements to a theoretical analysis of the concept of the individual in biology (my view of organisms as organic units). We can give a colloquial definition of organic systems as follows:

a) DNA is a hardware, which undergoes a selective pressure to optimise the function of the machine that transfers and modulates information (natural internal selection). b) DNA is ambiguous as the same sequence can be read in different ways. c) DNA cannot be considered as invariant because it is a structure "in flow", and it is intluenced by external (direct or indirect) sources, d) DNA sequences communicate in term of bijective homeostatic control. e) Genetic organisation is hierarchic as some genes control the others. f) Development, litness, and response to stress are regulated by internal and extcrnal signals differentially activating alternatc blocks of genes (Buiatti 1996).

The problem of causation in biology is a highly controversial topic. Here I do not want to deal with this issue in the history of biological epistemology (Mayr 1982:57-59, 1988:24-37; Nagel 1961). Let us consider a standard definition of cause: Cause is a nonsufficient condition without which an event would not have happened, or as a member of a set of jointly sufficient reasons without which the event would not happen. Can we accept that DNA has a causal value in biology?

As I have already said, if we accept genetic reductionism we have to consider DNA as a stable, coherent dimension. In fact, if DNA acts as the causal element in an organism's existence via mechanical physicochemical 
processes, its instability obstructs direct expression of a genetic endowment. In other words, if the phenotype is only the expression of a genome, and an organism's individuality is explained in its genetic data, it follows that phenotypic expression needs to refer to a stable dimension. Otherwise we cannot say that a genome will determine a certain individual. If we accept biological determinism, we have to conclude that since we (or other species) possess a body, the phenotypic expression of a genetic code has to be the coherent result of an unique and stable genetic sequence. If DNA changes, or is influenced by environmental factors, it will follow that we could not consider genomes as 'the' causal element of Life, and we should think of Life as a synergy of numerous biological processes. Can we defend this position?

Modern genetics has provided numerous biologic reasons to abandon the view thinking of DNA as the stable and coherent structure by which Life is expressed, inter alia: The Jumping Genes that change their place in the chemical structure of DNA, and influence protein production. The synonymous sequences that produce enzymes and the introns that influence these mechanisms in ways as yet unclear. All of these features of the genome give evidence of the variability of DNA. (We could also talk about the 'loose' relationship between genotype and phenotype that helps adapt the organism to environmental conditions.) Let us consider some examples. In 1989, the biologist Trifonof (1989:417-432) observed in DNA the presence of particular chemical sequences derived from evolutionary structures and having a functional meaning as yet unknown. These sequences conserved in DNA regions contain the chemical structure originating in Life. This means DNA structure is not entirely functional to a chemical purpose, but contains unknown processes. Other examples of complexity in DNA-expression are provided by the experiments of Lio' and Bagnoli's (1995:271-281). Both biologists studied the affirmations of anticodons in TRNA in organisms and concluded that we could not explain the affirmation of an optimal codon sequence as only an entropic mechanism. DNA function and structure cannot be explained only in mechanical terms. (Stanley 1994:244-253; Lio' 1995; Buiatti 1996.)

In natural phenomena, DNA functional ambiguity is also found in exon activity (Buiatti, 1996). Exon sequences can be shuffled by a mechanism of alternative recombination determining the creation of proteins having different functions. The number and quality of codified bases to form a protein can vary. This variation causes ectopic expression of different characters. Particular sequence orders can be noncoded for generations and 
successively translated impressively (Yokoyama, Calhoun, 1983). Or synonymous mutations can occur (take the case of GCU-GCC sequences in synthesising aniline) which distort the delicate three-dimensional design of the molecule, disturbing its capacity to carry out designated cell functions (Kimura 1980, Ridley 1985), and a populations' evolution. We can admit the existence of a process of exon recombination, and we can think of genetic identity as a "colloquial and plastic relationship organismenvironment" (Buiatti, 1996).

Another example of the impossibility of describing DNA as stable and invariant chemical information is the presence of jumping genes. In DNA extra genes exist (Dulbecco, 1995). Jumping genes have structures of repeated sequences (let us think of micro-satellite sequences). Some jumping gene sequences change their position in the deoxyribonucleic[ three-dimensional structure. Jumping genes disallow us from thinking of DNA as stable and coherent wholes of chemical information that get translated into a phenotype, and emphasise the plasticity of DNA.

Experiments on the response of plant genotypes to particular conditions of environmental stress (e.g. on Nicotiniana (Durante 1986:51-64), on tomato's tehylaton levels, and on osmotic stress and on fitness phenomena in cortex cells (Meins 1983:327-346), has impelled some biologists to take a new perspective. The variation of environmental factors causes an increase in adaptive reply by organisms in only one generation. This suggests that an interrelation exists causing genetic alternation as a consequence of the physical changes in nucleic structure (Waddington 1977). Additionally, replies to internal and external signals cause the activation of a single nucleotide block that determines a chain of events conductive to the greatest phenotypic fitness. This process is made by coadaptive phenomena between genotype and phenotype. (Other examples of genome plasticity are given in Nurminsky 1998:572574, Begun 1992:519-520; Long 1993:91-95.)

Two specific types of genetic control can be considered to emphasise phenotypic plasticity: allelic sensitivity and gene regulation. The allelic sensitivity is a genetic process by which the same gene differently influences its expression in different environments (single enzymes have different reactions at different $\mathrm{pH}$ value). The second mechanism shows that regulatory elements (genes) are sensitive to the environment and that they influence genes responsible for structural expression (Pigliucci \& Schlicting, 1993,1995). Given this, mechanisms involved in the phenotypic expression are qualitatively and structurally influenced by environmental factors, and this influence can change evolutionary trajectories. 
In considering the above data, we can hypothesise a mutual plasticity of both phenotype and genotype (and between both of them) - by an interaction of proteic series. Therefore obtain two important consequences: 1) we cannot define DNA as the invariant of Life; 2) we have to emphasise the organisms/environment dialogue in genotype-phenotype relation. Both these claims have been confirmed by scientific data resulting from the sequencing of the human genome (February 2001). As we will see in chapter II.2 of this dissertation, the low number of genes discovered in the human genome ( 30.000 genes instead of the expected 100.000, see Davies K. 2001) is now orienting the community of geneticists and biologists to explore the interactive relationship between genotype-phenotype (proteomics and developmental genetics) as well as the relationship between organisms and environment.

Both these conclusions persuade me to define organisms as complex entities that converse with the environment, changing their structural, qualitative and quantitative constitutions via this process. Clearly this view strongly opposes to genetic reductionism. In fact, the role of the genotype in an organism's biological existence is not basic. DNA gives chemical coordinates for protein formation, but its function does not explain an organism's existence, as the phenotypic influences have been shown to influence the DNA structure. In fact, the transmission of genetic information involved DNA transformations, since the creation of particular proteins refers to the choice of specific synonymous sequences and further chemical interactions ${ }^{9}$. This colloquial relationship is based on evolutionary and adaptive phenomena (Continenza \& Gagliasso, 1996), but this process does not immobilise the existence of an organism in an atemporal direction based on chemical translations of nucleus sequences. The biological existence of an organism refers to an open mechanism including the historical nature of organic systems - the present of an organism's existence as an individual, the past cumulated information and the future of its continuity through time (colloquial genotypic variations and transmissions in evolutionary dynamics).

The molecular biologist Wolf Reik has recently said (New Scientist, 28 November 1998 p. 29) that 'the constant emphasis on the power of genes has created a $20^{\text {th }}$-century form of fatalistic predestination in which people believe they are the product of their genes, nothing more, nothing less. Even geneticists have lost sight of the huge range of environmental factors that can change a gene's activity, ranging from an adult's diet to certain hightech fertility treatments. For those reasons, some geneticists are calling for a 
new definition of the gene, based on not only its DNA sequence, but also its epigenetic interaction manual - the degree of methylation for example". Genetic reductionism seems to be ending its domination of scientists' thought.

"Vicws on the evolution of Genome structure and function have changed dramatically over the past three decades. Once thought to be rather stable (...) the genome is now known to have a flexibility often referred to as plasticity. At the molecular level, the genome is like a puzzle made up of parts that can move from one position to another and, through exchange, deletion, insertion or amplification, generate new combinations of elements with different function and expression patterns". (Capy 1998:522-523)

Many reasons compel me to claim the limits of genetic reductionism. The impossibility of explaining biological individuality via an organism's DNA only is only one of them. Let us think of organisms/environment phenetic dialogue in evolutionary dynamics (Mayr 1981, 1988), or energetic changes between negentropic entropic systems (Prigogine and Stengers 1984, 1987). The first problem has been comprehensively analysed by Mayr, and I shall focus my analysis on the second point. I have stated that organisms are entities that have a negentropic feature (one-directionality), and that they are 'open systems'. Let us explain this paradox. Firstly, we have to distinguish between 'isolated', 'closed' and 'open' systems. Isolated systems cannot exchange energy or matter with the external world; closed systems can exchange energy but not matter, open systems can exchange energy and matter.

Organisms are 'open systems' because they exchange both matter (nurture) and energy (metabolism) with their environment. The organism's negentropy is related to the positive entropy poured by organism into environment. This mechanism causes a variation in the environment's total entropy that proves useful for an organism's survival. Organic systems need a continuous negentropic flow with the environment in order to increase the quantity of positive entropy used by the organism through time. Prigogine named these structure 'dissipative structures'. He said that organisms/environment communicate through a flux of energy. When these variations in the energetic flow occur, fluctuations in dissipative structures are produced. Organisms react to the above mentioned fluctuations by reorganising themselves. This mechanism is oriented to the growth complexity and energy consumption and it is based on environmental energy. Living organisms are dissipative structures - dynamic states of 
matter that originate in equilibrium conditions and involve an association between order and structure on the one hand, and dissipation (Tiezzi, 1984). If we think of biological individuals as dissipative structures, it follows that their existence refers not only to themselves, but is also based on their continuous energetic exchanges with their habitat. This flux of energy involves a dialogue-relation between living matter and environment. We can consider an organism as an entity that is strictly interrelated with its environment. In this view, an organism is a variable dependent on a set of variables whose sum defines a 'super-unity' - the biosphere. Organisms maintain certain relationships between 'transient states of their own structure and transient states of the world'. When we say that the genotype is the program for all an organism's features and teleonomic processes, we excessively simplify living matter's complexity. In fact, organisms possess two internal regulative programs - the genetic program and the somatic program (Mayr 1981). The information encoded in the genetic program is included in the somatic program by the ontogeny. Both programs are 'open' due of their energetic dialogue with the environment, and the absorption of the information contained in the phenotype ${ }^{10}$.

Evidence for gene-environment interactions at a molecular level are numerous (Wright 1998 and references therein). Genotype and environment interact and this mechanism is common in natural biological variation (Sultans 1987). On the other hand, it is well known that simple changes in the environment can affect genetic correlations (Campbell 1995). Both genetic plasticity and the organism-environment relationship are expressed in the organism's life and its continuity.

A mutual relation between genotype and phenotype exists. Numerous biological processes embody this mechanism such as expression of DNA, homeostatic control, metabolism, the immune system, encephalic trunk, and so on. This dialogue-relation ensures organism's unitary - and plastic Life, 'and' its dialogue with the environment. This mechanism is circular: [Genotype $\Leftrightarrow$ Phenotype] $\Leftrightarrow$ environment $\Leftrightarrow$ [Genotype $\Leftrightarrow$ Phenotype]. I call the relation between organism and environment R-relation. We can symbolise the organism / environment dialogue as follows: 


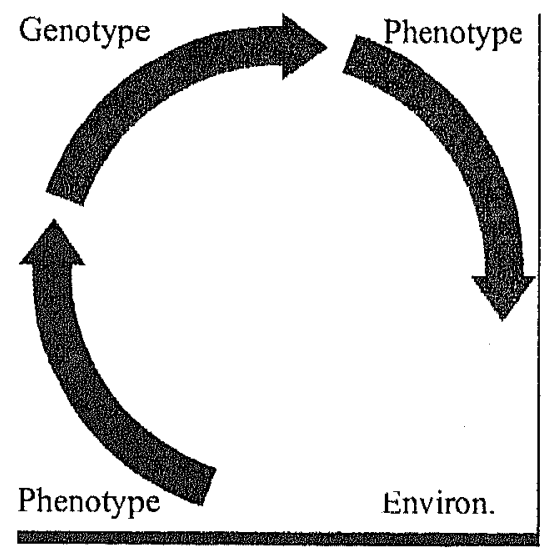

The reciprocal influence between organism-environment contains the possibility of DNA transformations caused by external (environmental) signals. It implies a genetic plasticity that has a fitness value.

In this chapter, however, I do not want to analyse the physical process of the organism / environment dialogue. I wish to stress the dialogue between biological individuals and their environment. If we accept the 'dissipative structures' concept, it follows that we have to include in the matrix regulating the organism's life (its environmental relationship) continuous energetic changes.

Although Prigogine denied that dissipative structures make a difference for biology, I guess that we can use this concept to stress the context environmental dependence of organism. In the pages of this dissertation I will often deal with this issue.

If we consider the organic unity view we see the r-relation, which joins the organism's heterogeneous and variable complexity in itself, also refers to environment. The organism's inner relation among its constituents - rrelation -.... is analogously extended to its environmental relations - Rrelation. (This property cannot be denied because an organism is a physical structure that interacts energetically with environment, and this process is confirmed by thermodynamics.) But this mechanism refers to a coherence mechanism - the relation between organism and environment, and organism's constituents aims at unifying and continuing a biological heterogeneity in a cohesive unity which varies in itself by remaining the 'same' plastic identity. On the basis of this, the identity-concept that posits 
an individual's existence as a process becomes clearer ${ }^{11}$. This involves that DNA ceases to be considered Life's 'hardware', but rather becomes part of Life's 'software'.

\subsection{Genetic Plasticity}

Dawkins advocated (1989) the view which considers DNA as software. He says that the phenotype has an evolutionary role and function - to safeguard and transmit genes. At the same time, genes contain adaptive information. Genes are the fundamental units of evolution, while natural selection is driven by the phenotype. The central claim of Dawkins is that since genes are contained in cells and these are the result of only one cell line (they are clones of the egg cell), the only strategy that genes could adopt for their multiplication was to safeguard their phenotypic 'container'. According to the British biologist, biological individuality becomes a genotype-phenotype coexistence. The genotype maximises the phenotypic peculiarities to safeguard the gene-container (the phenotype) through evolution. Natural evolution selects phenotypic variations to safeguard the continuity of particular gene pools. Genome interacts with phenotype to safeguard its continuity ${ }^{12}$.

Let us consider another epistemological root of the talkative DNA theory. During a conference at Berkeley, F. Jacob said that biological evolution presumes two laws: reproduction and interaction with environment. He suggests that we can think of organisms as open systems (Prigogine and Stengers, 1984). This view refers to the thermodynamic flow - organism's dialogue with the environment through a continuous change of matter, energy, and information. Jacob states that the environment has an active function in the selective processes. The relationship between genotypephenotype has fitness aims in evolutionary and generation terms. The continuity of this living matter's variability, influenced by environmental pressures, is guaranteed by a mechanism compacting the biologically unified variability (and its continuity) and assuring the unitary nature of organisms. This mechanism maintains the coherence of open systems in their plasticity and variability ${ }^{13}$. The open questions here are; What is the role of DNA in this process? Why should the relationship between organism and environment deny the gene-eye-perspective? Mechanists consider this relation contained within the organism's genome. If the relationship between genotype-phenotype is contingent to the individual's adaptability to 
its environment, DNA ceases to be considered as 'the' basic principle of Life. Its value is then limited to its functions in the synergetic process of Life $^{14}$. Since we cannot attribute a conditional value to organisms' constituents, it follows that the importance of the r-relation refers to its 'role' in the metric (logic) of existence of individuals. The agents of the $r$ relation are of no particular interest. They are numerous and composite, and they participate in the synergy of biological phenomena. This involves the denial of the possibility to approach natural phenomena via reductionism or mechanistic biology. The concept of individuality, then, does not coincide with that of genetic homogeneity ${ }^{15}$ (for example, in somatic embryogenesis). As Buss (1987) and Tauber (1992) have underlined, individuality is not a genetic 'sameness', it is a synergetic and complex phenomenon. An organism is a product of "its pre-history and external circumstances" (Tauber, 1992:129), and of continuous qualitative/structural variations influenced by environmental factors and oriented to adaptive finalities. The synergetic mechanism is a process of variation-in-stability, a homeostatic mechanism oriented to the future. Organisms maintain their integrity over time through continuous qualitative/structural variations. The organism's individuality is not destroyed by variability because organic systems act as whole-units, unified by causal mechanisms (such as the immune system, homeostatic system, central nervous system, cellular reproductions, and regulatory system). Organisms self-determine themselves via complex and synergetic interrelational phenomena within their constituents. These r-related systems participate to the organism's continuity through time. The role of organism's genetic identity in existence mechanisms thus is not exhaustive. DNA is only one of the numerous rrelated factors that assure an organism's life-continuity.

\subsection{Philosophical Implications}

What motivates genetic reductionism? Gilbert (1992) defines the knowledge of the complete gene sequence as the knowledge of "what it means to be human". Brenner (1982) claimed this knowledge could be used to synthesise artificial organisms. I will not criticise this hypothesis by underlining the actual heuristic level of genetics (DeSalle \& Linddley 1997, Lewontin 1998). I deal with this problem in chapter II.2 of this dissertation. Here, I shall analyse the conceptual implications of this paradigm. If DNA were the basic principle of life, it would follow that it would be the basic factor of living beings' existence. So, 1 am a 'human being', and my 
'essence 16 ، is explained by my genone. My genome contains my 'human' features. If this were correct, the knowledge of the living beings' genomes would offer humans the meaning of Life (Gilbert, 1992, Dulbecco, 1995). This is because Life is considered as a chemical physical process, nothing else.

In addition, interaction with this fundamental biological matrix would offer us the possibility to be the 'creator' of Life (Homo faber — Jonas 1987:615626). If DNA were to contain Life's essence, this would require it to have a primary value. Then DNA would be considered as the principle ex quo Life is regulated. Life would be based on mechanical factors - the physicochemical mechanism of DNA translation. Can we agree with this conclusion?

The primary question is why natural variability cannot be explained through a single property of living beings? We cannot attribute to a biological mechanism the role of first agent of Life. We can attribute a prevalence in terms of value to one specific property of living matter. We could think of it as the basic principle of natural phenomena. However, in doing this, we are then denying the variability and synergy of natural processes and the composite nature of living beings. Biological existence is a multidimensional system of diversities integrated into a unity. The phenotypic expression of DNA is influenced by environmental factors, shown in the bodily systems of physiology, neurology, immunity and metabolism. We cannot consider DNA's causal role a basic principle of natural phenomena. DNA is essential to biological phenomena, but its value is not basic ${ }^{17}$.

This means that I do not deny the causal role of DNA regulation in protein production, but the phenotypic expression of this chemical structure is the result of a number of (related) phenomena (nourishment, social adaptive environmental phenomena). Combined all together they express Life. An organism is a 'locus of functional and structural integration' or 'a system of integration within biological characters'. If it is a synergetic biological phenomenon, then mechanistic biology is unjustified. This objection refers also to the impossibility to define DNA as a stable and coherent structure, the relationship between phenotype and genotype, and the inexhaustibility of genome in natural phenomena. 


\subsection{The Unification of the Organic Unity}

We have seen that the concept of the individual is controversial in biology. A philosopher of science who explored this problem is David Hull. He synthesised the concept of the individual as follows:

\footnotetext{
"Individuals are spatiotemporaily localised entities that have reasonably sharp beginnings and endings in lime. Some individuals do not change much during the course of their existence. Others undergo, though limited, considerable change, and still others can change indefinitely until they eventually cease to exist. But regardless of the change that may occur, the entity nust exist continuously through time and maintain its internal organisation" (Hull 1980:313)
}

What continuity mechanism safeguards the individual's continuity regardless of its biological changes? Which property links the biological diversity of an individual to a unitary entity ${ }^{18}$ ? These questions demand an analysis of the causal mechanisms that unify an individual and regulate its continuity over time.

In the first chapter of this dissertation I stated that the organic unity concept refers to, inter alia, two conditions: maximum similarity and causal correlatedness. I would like to focus our analysis of identity on the cellular level, the so-called unity of organic systems. If we can justify the unitary nature of an organism at its micro-level, the unitary meaning of its existence in its macro-level will be evident. When we think of meiotic and mitotic processes, we can clearly see that cells possess a common requirement: a similar DNA. They are genetically homogenous ${ }^{19}$. This property ensures a high degree of similarity between and within individual's cells, so it is possible to say when two cells belong to the same organism. Cells are mutually interconnected, clones of the fertilised oocyte, and they will be connected with future cells generated by their continuous duplication. This property assures the causal correlatedness and similarity between the organic unity organism and its constituents (Salvi 1997:53-77).

Clearly when we talk about the causal correlatedness and similarity between organisms' constituents we stress the fact that heterogeneous constituents compose organisms. When cells differentiate they are not identical anymore. (They are similar.) In the sense that, for example, neural cells will be different from pancreatic cells and vice versa, while belonging to the same organism. Then one might think that the first condition to be an identity is cell differentiation. This would involve that, when organisms start to develop (totipotent cells) they are not 'individuals' and those organisms 
that do not have this cell differentiation cannot be considered as individual as well. But this is not true. If we think of embryogenesis we can have a better idea on this problem.

The first stage of an organism's development is the embryogenetic stage. The cells have not yet differentiated. Pre-embryo is a class of similar elements (undifferentiated cells) that increases progressively. These cells are blastocyts, clones of egg cells fertilised and replicated through time. If we define an organism $X$ as the sum of its constituents, we should think of its identity simply as the $\sum n$ (number of blastocyts at $t 1$ ) $x$-blastocyts. However, this conclusion is unacceptable because (1) $X$ is a variable identity strictly tied to time and (2) $X$ results from a causal relationship clustering existing $x$-cells.

This last point is crucial. First, causality in blastocyts means that they are clones of the same cell, and that they cause the existence of other $x$ constituents. Embryo cells are highly similar, yet concurrently they have the teleonomic function of forming a new organism. Depending on this program (regulated by the nucleic genetic information) an embryo develops. Blastocyts duplication and the difference in tissues are the phenomena of a biological process evolving through time. Within the pre-embryonic constituents are units that have genetic information. Within these operates the internal causal principle, pursuing the formation of the organism. As such, cellular sub-unity class $C(x)$, is not (only) a cellular whole tied by chemical or spatial, relations, but an evolving unicum. A fundamental link inside $C(x)$ blastocyts is the genetic code. DNA is an element (requirement) that confirms the internal causal relationship amongst the cell and the 'unitary' meaning of an embryo ${ }^{20}$. The information regulating the functions and the specialisation of tissues in an organism's cells is contained in the nucleic gene sequences. Mechanisms of genetic code translation (proteins) cause conduction mitotic phase processes conducive to cellular duplication. This is in relation to genome genetic information. DNA is a principle ensuring the continuity of embryonic cells. It transforms the $x$-class into a whole or unity. The pre-embryo example allows us to clarify the role of DNA in the existence of an organism. DNA has a causal role between organism's constituents at its micro-level. It ensures the continuity of an organism and links its cellular units. It would be unwise to consider DNA as 'the' causal principle of an organism, for an individual is comprised as a rich and complex integrated system. When the levels of biological complexity of an individual increase, the unifying mechanism becomes synergetic. Different systems will join the composite entity 'organism', such 
as, inter alia: (a) homeostatic processes, (b) DNA phenotypic expression processes, and (c) physiological mechanisms. The cohesiveness of the organic unity' is a 'property' of living beings that is not completely contained in the DNA. Since DNA is only one agent of cohesiveness (micro-level), I called this mechanism the r-relation ${ }^{21}$. As living beings are entities that vary over time, we have to link the r-relation to the biological variations of organisms. The relationship between environment and organism interacts with the biological constitution of organisms. This phenomenon has a fitness value. An individual will structurally and qualitatively transform itself over time, and continuously interact with its environment. At its micro-level think of energetic changes, and at its macrolevel think of changes in phenotype. The dialogue between organism and its environment is reflected in a phenotype-genotype relation, and ensures the adaptive tendency of living matter. The variation in this dialogue does not influence the existence of an organism, since this process is regulated by systems of coherent cohesiveness. We need to think of the organism as a system of biological diversities unifjed in a unitary entity. These systems guarantee the stability of Life towards environmental variability. Examples of these dialogues are homeostatic systems, immunological system, neurotopography, regulatory systems, etc. The unitary meaning of biological systems' unified complexity becomes the most instructive explanation for understanding their emergent properties. We should consider the organism's identity as an organic unity, that simultaneously changes yet remain the same. This plasticity is the result of continuous adaptive processes. In these terms the r-relation unifies the organism and ensures its continuity. This process is not a one-directional process but admits the plastic adaptability of organisms to habitats (R-relation). It is an 'open metric'. X's identity continues when the r-relation maintains its causal value - joining the organic unity on its own. If an organism's unity is more than the sum of its constituents, then the organic unity concept will need a fundamental condition. Why define an organism's identity as 'something else' other than the sum of its cells? Thinking of our own condition, the response is self-evident. We do not think of other living beings (say Mary) as simply a sum of cellular units. If we think of Mary as the physical presence of an individual, why would we consider the second example only as a cell culture? A maximal similarity and causal correlatedness relation correlate the cellular constituents. Since the organic unity is a unity-in-diversity, it follows that the degree of unification and the 
number of unified constituents denote the degree of complexity of the organic unity.

Take the case of an organism. We have cells, tissues, organs, systems, and a hierarchical organisation of biological constituents. An organism is a locus of functional and structural integration. This locus has a degree of complexity, which is also reinforced by the existence of psychological traits (consciousness through neural topographical structures). Cells in a test-tube do not have a sufficient degree of complexity of unified elements, so we have a 'whole-unity' contained in a test tube. We do not have an intercellular integration, nor the level of structural complexity needed to ensure a unitary meaning for the cell culture. We have a collection of cells that do not act (or behave) as a unity. Since an organic unity concept involves the impossibility of reducing the meaning of an organic unity to the sum of its own sub-requirements, it follows we can define differences among life forms by using this concept.

The organic unity concept implies that we emphasise the cohesiveness of the integrated heterogeneous complex organism on its own, using both biological and psychological features, as well as the causal joining mechanisms that unify the biological variability in a unitary dimension - the individual. According to this, the degree of unification of an organic unity depends on the complexity of its own unified constituents and the mechanisms of causal interconnection among the organisms' biological systems.

We can describe the organic unity's degree of unification in two ways:

1. We can consider the organic unity's degree of unification as a fixed parameter. In this case the degree of unification is related to the degree of diversity among heterogeneous constituents forming an organic unity - biological heterogeneity;

2. We can consider the organic unity's degree of unification as a variable parameter. This parameter varies depending on the variable degree of unification among heterogeneous constituents, which form an organic unity (the homeostatic processes, metabolism, netroanatomy, etc.)

Both these logical dynamics refer to a main feature of organic unity: an organic unity is a unified heterogeneous complexity. Since an organic unity, as we have seen, is a unity-in-diversity, the interconnection within its 
unified diversities and its unifying relations define its value (degree) of unification. In the second chapter I used the example of a picture to explain the organic unity concept. Looking now at a visual analogy:
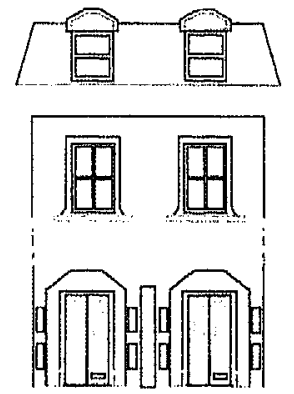

L $\quad$ j House A
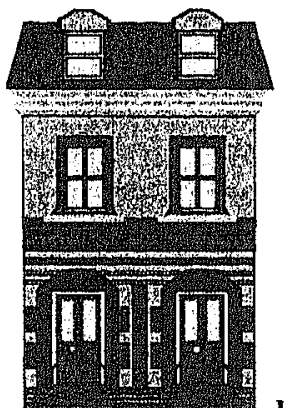

House B

The first image is a monochromatic picture (House $\mathrm{A}$ ); the second one is a polychromatic picture (House B). The unitary value of both these pictures is identical (they have a unitary value independent from the nature of their unified sub-constituents), but a difference between $A$ and $B$ exists. B possesses a polychromatic heterogeneity which A does not have. Its organic unity value increases depending on its unified heterogeneity (structural and qualitative diversity). In this sense, the organic unity's degree of unification refers to qualitative and quantitative diversities of its constituents, and the unifying processes compacting the organic unity on its own. The unification of an organic unity, is therefore a question of degree.

This criterion of classification systems can also be used in systematic biology to classify organic systems. When we consider the structural and qualitative heterogeneous diversity of an organism's constituents as well as the unifying processes that bound this heterogeneity in an unity (cellular duplication, biochemistry, homeostasis, metabolism, encephalic trunk, and the cerebral cortex), then we can define organisms' degree of complexity as follows:

The degree of complexity of an organism refers to the degree of the structural complexity of its biological constituents, and the degree of complexity of its clustering processes. This criterion of classification analyses the biological- mental features of an organism and its degree of unification. The degree of complexity of an organism is directly 
proportional to the complexity of its synergetic clustering-processes and the degree of unification of an organism on its own.

By means of the organic unity theory we may use either the organisms' structural features or their qualitative-functional features that would otherwise be excluded from the classification schema ${ }^{22}$. Even if we do not attribute to the organic unity model a strong enough value to constitute the filter and metric of a natural taxonomy, we will have to consider that all classificatory systems rely on a metric organisation. We cannot point out the difference between two things if we do not seek it, nor if we have no fixed reference-point in our comparative action. In this sense, the choice of a reference system becomes a primary concern for all classification systems.

The role of scientists is to define an objective reference that justifies its application to the investigated system. This property guarantees the coherence of a taxonomy. I have shown reasons that impel me to defend the organic unity model as the right theory to clarify the meaning of living beings. The use of this theory in systematic biology is an extension of this view of organisms in their universality. In parallel, the organic unity concept thinks of the individuality as a composite dimension that it cannot totally be reduced to some of its constitutive features arbitrarily chosen (such as the genetic identity). These indicative external references have been enriched by (though notmerely) the biological features that coparticipate in living beings' diachronic existence. According to this, taxonomy will consider the stochastic recurrences of these species-specific 'distinguishing marks.' 


\section{NOTES}

'The word 'invariance' means the power to reproduce and transmit - ne varietur correspondent information to the structure of organisms.

${ }^{2}$ The 'central dogma' of molecular genetics states that genetic information is transcribed into

RNA and then to proteins (DNA $\Rightarrow$ RNA $\Rightarrow$ proteins). The second law of thermodynamics proved the one-directionality of this process ${ }^{2}$. The information encoded in the DNA sequence of a gene can be transmitted along two principal pathways: (I) the cascade from DNA molecules to RNA molecules to protein, and (2) the circular route from one DNA molecule to another as the genes of a maturc cell replicate. This involves that genetic information flow is one-directional and irreversible, and that DNA structure guarantees the exact action of replication and translation.

3 "The seemingly irreducible or excess information is contained in the specific conditions of protein's environment, and only together with these can the genetic information determine unambiguously the structure and thus the function of the protein molecule" Monod, 1971:94. And this mechanistic properly of living matter is so strong to lead the French biologist to compare the biological systems to Boolean ones (input-output).

"In 'Il potere del progetto' (Șfera, n.43, 1995, pp, 11-34), Lewontin analysed the biological paradigm of cells-as-factory. This view considers organisms' existence as explained by their constituent proteins. DNA is considered the fundamental dimension of living beings' existencecontinuity. I utilise the Farm metaphor for exploring that biological theory that considers DNA the hardware of biological processes.

"If DNA changes, point (1) becomes nonsensical. TF" must accept the $X=X$ dynamics. This means that we consider a fixed quantity of nucleic sequences $Q(x)$ to completely explain $X$ 's identity. No variation of this clement is admitted, since the same metric on which $T F$ is built implies this constitutive hypothesis. If we have a variation within $Q(x)$ at $I 1$ (Let us call it $Q 2(x)$ ) and this change does not allow us to define $X=Q(x)$ (wc have to say $X=Q 2(x)$, we cannot accept the biunivocal property as the metric of X's continutity over time. How can we calculate $Q(x)$ ? Mutational phenomena induce numerous variations in organisms' constituents. Do we have to assume that $Q(x)$ is a statistic element, a recurrence of constant genetic data? If $X$ changes, it will be not true (or not completely true) that $X=X$. Given this, we ought to describe $X$ as $Q 2(x)$ or $Q 3(x)$ or ... If $Q(x)$ varies, should we use a multiplicity of genetic identities sharing a spatio-temporal dinension? Would it not be better to define in primis which meaning $Q(x)$ has in SI problems'?

"Walter Gilbert (1992) said that when we possess an heuristic level allowing us to know the complete genome sequence, we will know 'what it means to be humans'. Brenner is another gond cxample of radical reductionism. He claims that if he had the complete DNA sequence of an organism and a computer, he would be able to synthesise the organism (conference of the Darwin's death centenary, Darwin college, 1982). A recent criticism of this 'utopia' is DeSalle \& Linddley 1997

'Clearly this conclusion refers to TH"s idea that an organisms' phenotype is only a physicochemical translation of a DNA sequence. If this is true, two identical genomes will be expressed in two identical organisms. This means that monozygotic twins cannot be different in any way. They have to be identical in to each other. This is impossible since genetically and 
neural topographically their relation with environment will influence their existence. monozygotic twins are not idemical, but similar.

${ }^{*}$ If we consider $\mathrm{Gx}$, the genome of $X$ at 11 , and $G 2 x$ the same genome at 2 (atter two years for example), the relationship $6 x \neq(\mathrm{G} 2 \mathrm{x}$ impels us to drop the biunivocal property of $\mathrm{X}$. The same explanation can be given in considering mono-2ygote twins. If $X$ and $Y$ are the twins, we can say that $G x \neq G 2 x$ and $G y \neq G 2 y$. then we ought to conclude that $X$ does not continte in $Y$ and vice versa. If, as I argue, a relationship between genotype and phenotype cxists, we have to clarily its meaning and its consequences for the existence - continuity of an individual.

Take the case of junk DNA. Non-protein-coding sequences are responsible for countless biologic functions, such as contsol of gene expression, RN $\Lambda$ splicing, formation of chromatin domains, maintenance of chromosome structure, reconbination and replication.

to The impossibility of predicting the incidence of genetically induced epigenetic nutolions within natural evolution is still being debated in biology. In a recent paper, G. Vines criticised the claim of genetic reductionism that genetic inheritance has an exhanstive wole in an individuals' life (their plenotypic features as well as their qualitalive expressions). Dr. Vines stressed the impossibility of reducing inheritance to chenical processes. Organism's epigenesis reves a strict link between individuals and their environment. See: Vines 1998:26-30, Reik and Surani 1997, Jablonka \& Lamb 1995, Roemor 1997:277

"If must be stressed that the self is no way a well-defined (neither prodefined) sepertoire, a list of authorised molecules, but rather a set of viable states, of mutually compatible groupings, of dynamic patterns. (..) The self is not a static border in the shape space, delineating friend from foc. Moreover, the self is not a genetic constant. It bears the genetic makc-up of the individual and of its past history, while shaping itself along an unforeseen path" (Maturana \& Varela et at. 1980:363)

${ }_{12}$ Dawkins agrees with the idea that genotype and phenotype are interactively joined is unknown. However, the functional link between these elements in his view, suggests he could be considered as an early advocate of an interrelation between these two elements of organic systoms. It is not clear if a structural relationship between genotype-ptenotype can be altributed to Dawkins's viaw. However, I believe his theory constitutes are example of a relationship between genotype and phenotype.

${ }^{3}$ Mother nature as a tinker or bricolear. This ideo, which unites 3acob and Monod has ben criticised by Kaufmann 1992, and Goodwin 1983. They underlined the physical cvolution of biological systems. A recont defence of a similar idea is Bennet's 'meta engineering' in Dentett 1995:220:228

is Mechanists might objoct at this point. This not-yet-clear r-relation anong organism's constituents (and organism and/or enviromment) has a basic value in individual existence. Why not say that DNA has this function since the genome is the 'agent' of $R$ ? We can answer this objoction by saying that neither the genome nor $R$ completely explain the organic phenomena. $R$ urifics an organism $X$. It allows a distinction between $X(d o g)$ and $Y$ (hish). $R$ has this role because of its finction in identity mechanisms.

is "The ideal of the individual as an entity that may be treated as gengtically uniform is at bost an approxintation. It is apparent that individuality is a derived character, approximated closely only in curtain taxa". (Buss, 1987:20)

III am using this term in a reductionistic sense: essence as the basic element in my biological existence.

$"$ I do not chim that the knowledge of the buman genome will alicit the neaning of Lifi, I simply claim that this knowledge is one of the steps further that need to be taken into account 
to understand how Life expresses but it is not the basic element that can explain natural phenomena:

18 David Hull (1978) says "By 'individuals' I mean spatio-temporally localized cohesive and continuous entitics" (p.336); Leo Buss (1987) initially characterizes a biological individual as "a physiologically distinet discrete organism" (Preface, fin.2.).

19 The concept of genetic homogeneity is extremely controversial. The American philosopher de Sousa has summarised this puzzlo as follow: "What of genetic homogeneily? This requirement is puzzling for several reasons. Buss may mean simply that all cells are descended from a single zygote whose DNA pattern they share. This would preclude individuals arising from the fusion of two distinet organisms. Yet successful cases of parasitism or symbiosis are well known. But the passage would seem to imply, further, that since somatic cells are descended from a single zygote, they will not in general differ in their DNA. Since the function of the segregation ol the soma from the germ cells is precisely to proteet the latter from being affected by changes in the former, we must surely grant, however, that somatic cells may indiviclually lace mutations that will lead to diflerences in their DNA (...) The most plausible interpretation, I submit, is that genetic homogeneity acts as a marker of uniqueness, both synchronically and diachronically. $\Lambda$ any one tirne, it will be gencrally true (with the reservations just expressed) that any of our cells carries the mark of our complete genetic identity. But more importantly it is also diachronic: though Buss doesn't actually say so, he must surcly have in mind that only if the organism retains its genetic identity through time (...) in spite of what might be great morphological differences, especially in metamorphosing species can it be clearly marked off in its identity as well as its uniquencss from all other members of its species." de Sousa R. (1997) Biological Individuality in http://www.chass, uloronto.ca/-sousa/BIOINDIV.htm!

20 DNA is the property which ties the X's individuality in the transverse sense (the definition) and in the cross one (the evolution). It transforms the relationslip among the $C(x)$ constituents, as a relationship absorbing in itself causal consequences that difterent temporal phases have in development.

${ }^{21}$ The r-relation is a set of unifying factors clustering the biological diversity in a locus of integrated complexity. It links the relationship between genotype and phenotype, and both participate in the phenomenon of an organism's self-unification.

22 This means that since self-awareness consolidates an organism's organic unity. Those organisms that have mental-traits will reinforee their structural biological complexity with their psychological complexity. They have a higher degree of unification than pre-conscious organisms. According to this, a plant, for example, will have a minor degree of organic unity in comparison to a mammal. The plant is without neuro-structures (neuro-phenomena) and its biological apparatus is not as complex as that of mammals. 


\section{I.3 Plasticity through time}

In the previous chapters I stressed the complexity of biological phenomena. This chapter of my dissertation aims to defend a view based on an organism's unified complexity. I will extend the organic unity theory to incorporate species-specific identity. I will argue that species are an organic unity based on conditions of maximum similarity and causal correlatedness, as well as on biological variations (the evolutionary mechanisms).

\subsection{Taxonomic Problems: How To Classify Living Beings}

The species results from a classification system that links the biological peculiarities of organisms within unified taxonomic classes. Species are groups of organisms that have similar features to each other (similarity ${ }^{1}$ ), and which are capable of breeding with each other (inner causality between its members). A species is a natural population that has reproductive unions and is isolated from other groups. Questions arising from this are: Is a species an identity? What property joins organisms to an existing species?

I look at my dog who is named 'Pluto'. I know that he is both a biological individual, and a member of a specific animal species - the canines. Pluto shares a large number of phenetic similarities with dogs classified as German Shepherds, so I call him a German Shepherd. I classify Pluto through a classification criterion based on the comparative analysis that when organisms are 'sufficiently' similar, they belong to the same class. I called Pluto a 'dog', so I am still accepting the infra-species classification system used by biologists. Why do I accept this? Why cannot I employ 'my' biological taxonomy? Which classification criterion do biologists use ${ }^{2}$ ? Is the species concept really unproblematic?

The classification of something is an act external to classified objects. It is a mechanism depending on a referent (an observer) and a classification system (referential system). Organisms are classified in composite units via outside references by human beings. So we need to acknowledge valid reasons by which the acceptance of an organism to a certain biological class (species) is justified. These parameters refer to the observer's weightings system in their taxonomic act. 
Countless descriptive and evaluative systems exist to describe objects. When I look a stone I could say, 'this is a stone because it possesses an atomic configuration and a particular set of physical features that impel me to catalogue it with all the other stones which posses these features'. If I then change my reference system, for example, I classify objects via their form, then my classification varies. I could include tennis balls and round stones in the same class. This is a different classification, not a wrong one. It refers to different referential elements. Taxonomy thus is a relative mechanism based on (variable) criteria of classification used by observers.

It is important to stress the limits of the classification processes since biologists use different systems of classification in defining species. (On the problem of biological classification see: Mayr 1988:268-288; Sokal 1963; Simpson 1961; Wagner 1969.) One of these systems of classification is based on phenetic similarities (we need to be clear if this system uses the organisms' qualitative or functional peculiarities), and another is based on phylogenies (organisms are classified because they descend from a common ancestor). One must be clear on one's classificatory system, otherwise I can object to the above mentioned systems and classify 'dogs' by their hair ('Hippie dogs', 'Rasta dogs', or 'Marine dogs') or deny the need of a biological classification at all if I consider Pluto as an individual absolutely unique in his make-up ${ }^{3}$.

I do not want to analyse the meta-problems of systematic biology here (Sokal 1963, Jonson 1967, Hartigan 1975, Shepard 1962:125-40, 1979:87123). I only aim to underline the logical dynamics of classification-systems. We may describe a clustering mechanism of classification systems as follow ${ }^{4}$ :

1. I identify in an organism $(X)$ a set of peculiarities $P(x)$ which label it as individual

2. I make (1) in other organisms;

3. I use a criterion of classification by which I classify organisms in groups because they have a whole $T$ of factors $P$. I call these classes TPs;

4. Comparing $P(x)$ with TPs $I$ determine if $X$ is a member of TP.

When 1 do not want to use the classification system 'Hippie and Rasta dogs', I have to attribute to my schema a third point of normative value. I then accept the systematics of biologists (for example) in order to define a universal system of biological classifications. My classification will refer to 
this universal taxonomic system ${ }^{5}$. In doing this I conclude: Pluto is a mammal, a dog, a German Shepherd, and 'the' dog named Pluto. Which taxonomy should we accept? Do we need to analyse the genotypic or phenotypic similarities? How is Pluto's individuality explained by its species?

\subsection{The Species Concept in Biology}

In Chapter I.1. I examined the classification of points in a surface. We saw that they were of a complex classification - a set of vehicles (bicycles, motorbikes, and automobiles). The classification is similar to the one in the preceding paragraph. I will describe their peculiarities (a motor, two wheels, and so on) in order to classify objects' features. When the number of these peculiarities increase, the descriptive precision of our classification grows. If we restrict the classification of the vehicles to the condition 'having two wheels', bicycles, scooters, and motorbikes will belong to the same set. If we wanted to isolate motorbikes as a separate classification then we have to find their distinctive specific features. When the structural complexity of the investigated objects increases, we need to describe their individual specific features. A classification that is precise enough to define the set 'motorbikes', needs the construction of careful description. If the complexity of investigated objects is high enough to make truly 'accurate' descriptions difficult, how are we to classify them? (If we think of living beings this problem becomes pivotal. Since organisms are loci of integrated complexity, which level of their morphological complexity should be analysed in my taxonomy? (Ghiselin, 1966: 207-215; Hull, 1976:174-191, Lewontin 1998)

We can use another criterion of classification, that of functions as an expression of a feature. We base our classification on particular functions made by members of a specific set when we recognise that these functions are the expression of their feature. In the case of motorbikes, I can say; 'I am looking for objects with two wheels and a motor which provokes them to spin'. I therefore insert a functional distinction among vehicles with two wheels by adding to this condition another regarding a motor's function. In doing so, I change my classificatory perspective from a descriptive level (the requirements) to a functional one (what function do these requirements have).

These classification dynamics can be applied to biology. Organisms are structures of integrated complexity. The heterogeneity of living beings is so 
high that the description of their structural peculiarities can continue ad libitum. I can think of their genotypic peculiarities, phenetic peculiarities, homologies of organs etc. This constitutive complexity pushes us to look for features that can help us in their classification. This means that in systematic biology the qualitative classification is linked to the functional description. As we should add a functional condition to classify motorbikes (to avoid overlapping motorbikes to bicycles) systematic biology finds qualitative and functional similarities among organisms and bases its classification on a referential element (the reproductive clause) linking organisms in units strictly unified in their own. The organisms' qualitative peculiarities are their biological features, and the organisms' functional peculiarities are the biological functions of these requirements.

In systematic biology, morphological similarities allow us to carry out an initial classification (phenetic or phylogenic classification), but this initial taxonomy has to be enriched by a causal property, which strictly separates the members of a species, by themselves: to be reproductively isolated from the other populations. This property ensures the causal correlatedness within the members of an isolated whole.

One conclusion of this analysis is that in systematic biology we follow a bipolar dynamic. We describe the qualitative features of organisms (the conditions of maximum similarity), then we investigate if their functionality causally joins them into separate units, ${ }^{6}$ which is the condition of mutual causality. The mutual causality within members of a species is really problematic because it involves genealogical relations among the members of a class (Hull 1976, 1978, 1987; Kitcher 1984, 1987). The historical continuity of a species refers to lineage continuity. This involves that all species are in a continuous state of genetic flux. The notion of lineage is only a "biological" way of stressing the species continuity problem ${ }^{7}$. The species continuity involves, per se, all the above mentioned mechanisms 8 . The biological concept of species is based on population biology. In this view the species are (1) a whole of populations, (2) all populations form a species when their members maintain the possibility of reproduction with fecund progeny and, (3) each whole is, reproductively, isolated from the other wholes of populations (Mayr 1981:23). The first aim of biologists is to cluster organisms that have common peculiarities in composite classes. From this perspective "a typological species is an entity that differs from 
other species by constant diagnostic features" (Mayr, 1981:316). The first deficiency of this view refers to the discovery of a high frequency of morphologically indistinguishable species ('sibling species'). This indicates that typological features are not sufficiently defined conditions for a species' identity. Therefore, we need further mechanisms of classification. This further taxonomic step is the species-specific organisms' capacity to reproduce themselves despite their phenetic resemblance. (Species have the capacity to evolve because organisms have the capacity to reproduce. Wilson, 1995:353; Hull 1978:341) I can then define the canine race by excluding classes of organisms that cannot mate with dogs (chickens for example). I call this conditional property the c-condition. The c-condition is the capacity of 'reproduction with fecund progeny' and is a causal principle by which the species-specific members are related.

This property refers to the species' historical continuity. Members of a species can mate over successive generations. This property links members of a species in a causal sense".

\subsection{Reproduction in Systematic Biology}

Since this dissertation aims to investigate concept of the individual in biology, I will not analyse the different biological taxonomies. Instead, I will 'a-critically' accept the species standard-concept in population biology; species as a whole of populations, reproductively isolated from the others. Let us consider another example, this time that of a horse and a donkey. They are similar, but do not belong to the same species because their progeny (the mule) is sterile. Even if their phenetic similarity is high, this does not constitute a sufficient condition to ensure that they belong to the same species. This means that their structural similarities (for example, DNA structurc) are insignificant in systematic biology, because the ccondition's causal role has a prevalent value.

As we cannot explain an organism's identity via its genotype ${ }^{10}$, we have to consider the species-specific identity through its heterogeneous nature. The causal link within members of a species is not simply any particular feature of their biological constitution, it is the functional expression of their biological constitution -c-condition. This property denotes the speciesidentity in a causal sense and enriches the condition of 'maximal sinilarity'. Mechanists could object that infra-species similarity refers to genetic similarity. Why cannot we classify species via genetic similarities? If species are composed by organisms joining a species-specific gene pool that 
will be inherited by descendants, it follows that we could identify the species in a specific genetic lineage. We could consider this element the 'basic element' of a species (Kitts 1984:112-115). We can reject the conclusion that the invariability of essences is completely different to the biological (evolutionary) propensity to change (Mayr 1981:345). We could object that a 'specific-genetic similarity' is a property resulting from a comparative analysis amongst members of a species (their typological features). A referential property that clusters the members of a class could not be considered their 'basic feature', because we can use other classification-systems to find different topological similarities. We could also use the heterogeneity of organisms and the equivalent relativity of classification-criteria to show the incoherence of a classification-process passing from a descriptive level to a contingent one. The evolution of a species-specific genetic pool, in this sense, shows the inconsistency of this view (otherwise we have to think of essences as variable dimensions). At the same time, if we think of the genotype as the referential element in systematic biology, we have to describe the similarity among infra-species' individuals via a comparative analysis of their genotypes. How can we define interspecific genetic boundaries? What is the relation between genetic individuality and a species genetic identity? Is genetic conformity the condition to belong to a species?

If we consider that infra-species' individuals are historical entities that change through time, then the synchronic definition of a species implies a constitutive variation within its sub-elements. According to genetic essentialism we ought to think of species as eternal types. This conclusion is obviously false. Evolutionary mechanisms show a continuous mechanism of animal transformations, so we can then consider the causal condition, (in reproduction terms), as joining infra-species' individuals".

The species' historical nature reinforces this conclusion. Also, varying species maintain a quantity of information ensuring the constitutive similarity among its members. Consider the following figure: 


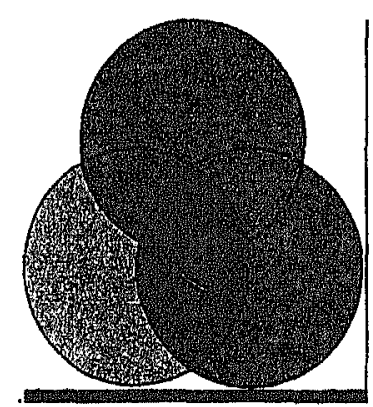

Suppose each circle refers to the genome of a single individual. The common spatial surface within the circles individuates the common information encoded in their DNA. In addition, DNA is the vector of this transmission, and the element that assures the species continuity. The peculiarities of a species, however, are not completely contained in its DNA.

The members of a species link their constitutive diversity to an internal similarity of genotype and phenotype. They share a set of genetic information. This property ensures their internal similarity. Species-specific gene pools, even if they do not contain species-specific identity, have a role of causal interreletadeness among species specific individuals. On the other hand, we have seen that the similarity-condition is an unreliable explanatory tool to define whether an organism belongs to a species. This unreliability is mainly due to the fact that biological individuals have an unique genome. Furthermore, it is treacherously difficult to define a boundary of similarity explaining why an individual belongs to its species. Finally, difficulties arise because the c-condition refers to phenetic features of living beings. This means we have no reason to make species specific identity contingent to a particular DNA, or to consider the species' continuity only as a chemical phenomenon.

\subsection{Analytical Approach to the Species.}

Take the case of organism $X$. Its species is the whole $U G(x)$. We could define this composite identity as the sum of its own constituents, or as a composite organic unity. Let us analyse the first hypothesis. If a species is the sum of its own constituents, then $U G(x)=\sum n X(1<n<+\infty)$. This whole has an indefinite margin of internal variations, and respects the $c$ - 
condition (when we do not respect this condition the identity of $U G(x)$ is bifurcated). $U G(x)$ is not explained by some of X's particular requirements (topological similarities), but is based on a r-relation that links its constituents. How can we define the cohesive r-relation of $U G(x)$ ?

Using our common sense, when we think of a species we do not think of a mathematical class $\left[U G(x)=\sum n X(1<n<+\infty)\right]$. What reasons would induce us to think of a species as a heterogeneous and composite entity whose nature is not explained in its numerical description? Let us approach this problem analytically. The species is 'a further element' other than the sum of its members ( $\left.U G(x)=\sum n X\right)$. The concept of species refers to two problems: How can we define the nature of a species in itself? How does a species continue over the course of time ${ }^{12}$ ?

A species is a composite dimension of organisms having a topologic similarity and causal correlatedness (the reproductive property). At the same time, these organisms have a spatiotemporal relation (organisms live in a specific spatiotemporal system). We can clearly see that a species can be considered a whole-unity (chapter 1.1.2.) because of the relation of states, that join its sub-elements. In parallel, the numerical and qualitative variations of its members define its nature as a whole. We can thus conclude that a species is a whole-unity.

The homogeneous nature of a species' integrated complexity is unified by the causal interconnection between its members (the c-condition). This property links the members of a species by a relation of causal correlatedness (organisms are generated by others, and they are capable of interbreeding), and by a constitutive similarity (orgatisms respect the ccondition because they have the 'same' reproductive apparatus). At the same time, a species-identity is 'something other' than the sum of its members or the c-condition. Why is it essential that we can intuitively know that the species-specific identity is not the sum of its members?

\subsection{Species as an Organic Unity}

Here, I will claim that the species concept is analogous to the concept of the 'individual-nation' as scripted by Hume. As the concept of a nation incorporates territory, people, history, political peculiarities, and is not limited to only one of these elements, species specific identity refers to itself and is not explained by some its own (qualitative of functional) peculiarities. A species, therefore, is an organic unity. 
Two reasons lead me to think of $\operatorname{UG}(\mathrm{x})$ as an organic unity. The first is logical, the second conceptual. In the first chapter of this dissertation I defended the thesis that an organism $X$ is a organic unity. As $U G(x)$ is composed by $\sum \mathrm{nX}$, it follows that a species is an organic unity (because individual organic units compose it). This concludes that since the whole UG $(x)$ is constituted by biological organic units, their properties are extended to the whole on its own. This conclusion can be rejected if we do not accept the syllogistic logic (in the set $U G(x)=\sum n X$, the first element has a common property. Since $\sum \mathrm{nX}$ is a whole of organic units it follows that this property will also connote the set $U G(x))$. I am aware that this is an inferential argument. In order to think of $U G(x)$ as an organic unity we need to understand that $\mathrm{UG}(\mathrm{x})$ is a whole of sub-elements $\mathrm{nX}$ interconnected by a causal r-relation. The r-relation compacts the whole UG $(x)$ into a unitary entity, which has an integrated complexity. This unicum is not explained by the sum of $n X$, rather, it is an autonomous identity.

First, let us stress the causal r-relation among $\mathrm{Xn}$ individuals. I argued that the condition linking the members of a species is their qualitative and functional topological similarity. The similarity concept does not involve their coincidence. The organisms of a species are not equals $(X \neq X 1 \neq X 2 \neq$ $X 3 \neq \ldots . . . X n \Rightarrow U G(x) \neq \Sigma X n)$. This means that a margin of differences exists between $\mathrm{nX}$ individuals. Species-specific organisms belong to the same species because they have similar biological identities.

This condition of similarity refers to constitutive and functional biological similarities among a species' constituents. The species-specific organisms' possible dissimilarities refer to their constitutive similarities. This is a constitutive property of species ${ }^{13}$ and refers to phenetic and genotypic differences ${ }^{14}$. The former are the ectopic expression of genetic characters (the expression of nucleic sequences) whose role is to increase the individual's specific fitness value (and the species-specific fitness value), the latter aims at producing genetic differences to improve adaptive phenomena. Therefore, infra-species diversity refers to an organism's genotype $\Leftrightarrow$ phenotype. This mechanism allows for natural evolution. Biological diversity is an intrinsic property of living beings, and refers to numerous biological phenomena such as crossing over, sexual reproduction, mitotic and meiotic processes, mutations, dialogue between genotype and phenotype, fitness, natural selection, evolution ${ }^{15}$, and so on. In addition, the longitudinal identity of a species implies a mechanism of continuous 
variation of its elements. This property denies the possibility of considering a species as the sum of its individual organisms. The species, as a nation, is a composite entity that cannot be explained by the sum of its members. The species is an historical variable entity that alters over time and whose identity is conditioned by evolutionary and fitness phenomena.

We have seen that the species is unified by mechanisms of (1) maximal similarity among its members and, (2) causal correlatedness. The first requirement allows the use of the species concept as classes. However, since the membership of a class is determined on the basis of similarity, we cannot limit the species concept to this class concept.

The causal correlatedness of the species-reproductive-community clusters the species in an 'cohesive-variable' unity-in-complexity. It unifies it into an organic unity.

The continuity of both species-specific individuals and species-continuity, is a problem that becomes central to an analysis of the species' (and individual's) individuality ${ }^{16}$. This is because species exist at a certain time and through time. These dynamics are correlated because of their similar definition, where a species is seen as a set of organisms that are linked by a relation of causal correlatedness and similarity. This observes their qualitative 'non-coincidence'. Simultaneously, the condition of causal correlatedness that unifies the organic-unity species' ${ }^{17}$ is oriented to the future in order to respect evolutionary phenomena. We can therefore synthesise the above conclusions as follows:

A Species is a whole-unity of organisms with an internal r-relation that joins them into a global organic unity. The r-relation is the element that ensures the species coherence throughout time. Its identity is not exhaustible either in r-relation, or in its sub-elements. Its identity is analogous to the 'individual nation' view of Hume.

Since a species unifies itself through time by its r-relation, the weight attributed to particular or unique requirements of a species can vary. Science is the continuous change of paradigmatic views (Kuhn, 1962; Salvi 1996). The species concept, such as topological entities, and species-specific gene pool, becomes insignificant if a species seems to constitute an identity on its own. This identity is not contained in its requirements, or in its features. Species-requirements are contained in an entity, which is analogous to the concept of the individual-nation of Hume. As species are organic units it follows that: 
1) A species existence is oriented to its continuity

2) A relation of causal correlatedness and constitutive similarity interconnects members of a species

3) A species' identity is not explained by $\sum n X$

4) A species identity is an organic unity

We can think of species as individual entities ${ }^{18}$ by using the Latin concept where an individual is an indivisible entity. An individual organism is a composite identity with a strong integration among its constituents. Likewise a species would have a heterogeneous integrated unity in their composite identity. The co-existence of an integrated group of individuals is reinforced by more than just biological clustering factors. Let us think of meme theory ${ }^{19}$.

Take the case of human species. We have endless reasons to say that the human species is something other than the sum of human beings living in the world. Intuitively, we think of 'humanity' as something distinctly different, something other, than a set of human beings ${ }^{20}$. How do we describe this difference? Evolutionary, biological, and cultural phenomena ${ }^{21}$ reinforce human awareness to form to global unity. Cultural, social, technological, and historical phenomena make humanity a unified identity $^{22}$.

A species has biological peculiarities inherited by descent. However, strong processes of non-biological interrelation exist where the species-members pass on the heritage of behavioural information ${ }^{23}$. In humans, this mechanism is by cultural transmission. Transmission of knowledge develops the human consciousness so that it belongs to a composite class of individuals. It increases species-awareness, a process that is very important to organisms with a secondary consciousness. This indicates that culture has a causal role in a species' persistence over time, because it unifies the species in itself. In animals this transmission is by genetic information, imprinting phenomena and instinctual behaviours. In human beings these factors are enriched by culture. When the strength and the role of this unifying process varies, so too does the degree of complexity of the global unity species. The degree of these organic units refers to their degree of cohesiveness and complexity. Since the organic unity is a unity-in-diversity, the increase of the unified properties-peculiarities of a whole-unity will reinforce the degree of cohesiveness of the organic unity in its individual- 
level (the organism) and in the global-level (the species). The organic unity theory thus in population level can be synthesised as follows:

\begin{tabular}{|c|c|c|}
\hline & Structural features & Qualitative features \\
\hline $\begin{array}{l}\text { Inner } \\
\text { Causality }\end{array}$ & $\begin{array}{l}\text { Species-specitic organisms } \\
\text { helong to the same species and } \\
\text { have interbreeding capacity. }\end{array}$ & $\begin{array}{l}\text { Species-specific organisms continue in } \\
\text { evolutionary time by receiving- } \\
\text { transmiting the biological information } \\
\text { to perceive best fitness finality. }\end{array}$ \\
\hline $\begin{array}{l}\text { Inner } \\
\text { Similarity }\end{array}$ & $\begin{array}{l}\text { Species-specitic organisins } \\
\text { have a species-specific gene } \\
\text { pool and a structural (variable) } \\
\text { similarity. }\end{array}$ & $\begin{array}{l}\text { Organisms' constituents have an infra- } \\
\text { specics biological diversity oriented to } \\
\text { fitness and evolutionary purposes }\end{array}$ \\
\hline
\end{tabular}

Homeostatic The species specific biological The species is a homeostatic structure Function structure is oriented either to and adapts its inner variability-in. the individual life of its own stability to environmental pressures. members or the species contituuity

Unitary Entity The species is a cohesive The species is composite entity, which dimension of heterogeneous links itsell to environment and constituents, shating a space maintains a continuity on its own. time system and lorming an unitary locus of structural integrated complexity

\section{NOTES}

I am not advocating cladistic classification (Cracrah 1987, Nixon and Wheller 1990). As Hull (1979) pointed out, cladistic analysis classilies characters rather than organisms. Clearly my emphasis of the heterogencity of speeies does not attempt to defend a criterion of classitication based on similarity within species specific individuals. To the contrary: it aims to defend a biokgical taxonomy based on both causality and similarity concepts. But similarity docs not have a basic value among the members of a population. It simply displays a constitutive similarity that allows the inter-reproductive capacity among members of a population. The similarity clause thus is addressed to maintain the causal interrelation anong the individuals of a species. This involves that my taxomomy per se denies the prevalence of a determinate weighting dimension attributed to some species-specific individuals leatures. On this topic see: Ilull 1979; 1976, Ghisellin 1974:536-544.

2 "One side (with Sebwartz and Ruse) says this: To know what a species really is, find the properties that constitute necessary and sufficient conditions for belonging to that species. In practice, of course, we may identify the bearers of those properties in terms of causul-relational propertics of lineages. For those - or so Darwinian theory leads us to expect - explain low things of that kind got to have their characteristic properties in common. These causalrelational properties, therefore, will be good though not infaltible predietors of the essential 
1) A species existence is oriented to its continuity

2) A relation of causal correlatedness and constitutive similarity interconnects members of a species

3) A species' identity is not explained by $\Sigma n X$

4) A species identity is an organic unity

We can think of species as individual entities ${ }^{18}$ by using the Latin concept where an individual is an indivisible entity. An individual organism is a composite identity with a strong integration among its constituents. Likewise a species would have a heterogeneous integrated unity in their composite identity. The co-existence of an integrated group of individuals is reinforced by more than just biological clustering factors. Let us think of meme theory 19 .

Take the case of human species. We have endless reasons to say that the human species is something other than the sum of human beings living in the world. Intuitively, we think of 'humanity' as something distinctly different, something other, than a set of human beings ${ }^{20}$. How do we describe this difference? Evolutionary, biological, and cultural phenomena ${ }^{21}$ reinforce human awareness to form to global unity. Cultural, social, technological, and historical phenomena make humanity a unified identity $^{22}$.

A species has biological peculiarities inherited by descent. However, strong processes of non-biological interrelation exist where the species-members pass on the heritage of behavioural information ${ }^{23}$. In humans, this mechanism is by cultural transmission. Transmission of knowledge develops the human consciousness so that it belongs to a composite class of individuals. It increases species-awareness, a process that is very important to organisms with a secondary consciousness. This indicates that culture has a causal role in a species' persistence over time, because it unifies the species in itself. In animals this transmission is by genetic information, imprinting phenomena and instinctual behaviours. In human beings these factors are enriched by culture. When the strength and the role of this unifying process varies, so too does the degree of complexity of the global unity species. The degree of these organic units refers to their degree of cohesiveness and complexity. Since the organic unity is a unity-in-diversity, the increase of the unified properties-peculiarities of a whole-unity will reinforce the degree of cohesiveness of the organic unity in its individual- 
properties definitive of any given species. But the causal-relational properties will provide merely contingent idexistence of a class essentially defined by essential properties. The other side (with Hull and Ghiselin) sends back a mirror image of the first. They argue as follows: To know what a species really is, you nced to find the lineage to which its nembers belong. That is, you need to find a group related by certain causal links to some given ancestor. In practice, of coursc, we may identify the lineages in question in terms of certain properties .... similarities and common features - which will be good though not infallible predictors of the relevant causal-relational properties. Bul those common features will provide merely contingent idexistence of what is essentially an individual, defined by its causal-relational properties." (De Sousa 1989)

"I might detend the thesis that species do not exist in nature because individuals are separate identities. This concept has been presented by the so-called nominalists. See: Gilmour 1940:461-474; Kitcher 1984:308-333; Ehrlich \& Holm 1962:1228-1232, Burma 1949:369. 370 .

"Clearly in biology other dynamics have also been used: Let us think of the phylogcnic,

phenetic, genetic, ecologic approaches to the species concept here explored.

The unit of evolution is one of the basic problems of modern evolutionary theories (Hull 1980, Ghisclin 1974, Brandon and Burian 1984). As Khalil (1997:197-199) pointed out, we can approach this problem through juxtaposed approaches: a phenomist sense (subspecies, specics, genus class), an ontological sense (individuals, troops of organisms, organisms), or a naturalistic sense. In this dissertation I will use the naturalistic approach to the species problem. According to this I will ernphasise the species problem in biology through efficient causal relations linking members in a population, and in organjsms themselves. I attempt to defend a species concept connoted in rational terms, because ontological or metaphysical views of the species problem can be criticised for their axiomatic basis, or simply refuted for being nonscientific arguments.

'Depending on the valuc attributed to organisms' features, our classification will vary (think of the difference between phenetic and phylogenic classitication). The choice of these values depends on the aims of nur classification. If we attempt to study the evolutionary genealogy of a species it will be considered its phylogeny; if we attempt to observe the species' features (for exanple behaviours) we will stress other species-specific traits.

${ }^{7}$ We can talk about a gene lineage (Dawkins 1982), species lineage ("species is a single lincage (..) which occupics an adaptive zone minimally different from that of other lineages in its range and which evolves separately from all lineages outside its range" Van Valen, 1976:233); population lineage (lineage as "spatiotemporal sequences of entities that causally produce one another" Hull, 1981:16), monophyletic lineage (lineage as "a set of entities that share a particular ancestor-descendent relationship unique to themselves" Brandom 1984, Horvat 1997:226-228), and so on. We can also consider lineage as "a sequence of reproducing entities, causally related to one another via reproduclion" (Wilson, 1995:341).

"The problem occurs when we make one of these "lineage-features" absolute. For example, an analysis of specics continuity over time based on genealogical relutions betwecn species individuals, risks to neglect the historical nature of species. It stresses the present-past relation. and neglects the present-future relation. Since organisms and species are historical entities, we should look for continuity conditions addressed to the future, similar to those that address the past. This is because organisms result from a lineage (descendant relations), but they will project their lincage through time (Wilson, 1995:342). The impossibility of separating the fulure from the past of an organism neglects the genealogical approach to the lineage notion. 
This does not mean that we do not need to analyse the paleobiological relations, it simply means that the species continuity is a logical mechanism based on reproduction and evolution. When we identify this causal process into a determinate weighting system, we make contingent a composite mechanism on one of the biological features of species continuity over time. Even if this approach is correct, we cannot think of it as an exhaustive explanation of species continuily.)

${ }^{9}$ Clearly, if genetic mutations obstruct the C-condition, the parental species' identity will be bifurcated in two (or more) different species. The new species, however, has to respect the Ccondition (be reproductively isolated from other populations.)

${ }^{10}$ If we link these considerations to the problem of DNA valuc in biological identity, it follows that since organisms reproduce via phenotype, and genotype is only a 'requirement' of organisms, that individuals belong to their species via (and because of the co-existence of genotype and phenotype. DN $\Lambda$ is not the basic reference used to classify species.

This involves that the genetic similarities joining the members of a population (speciesspecilic gene pool) are functional with respect of the reproduction clause. Even if the genetic consistency of a given population exists, this homogeneous element involves per se a historical variation (evolutionary mechanisms), and a genetic diversitication within the members of a species. The sexual reproduction mechanisms guarantee genetic diversification of the progeny relative to the parents, thus they connote reproduction in terms of genetic variability. When we think of a specics as genetic types, we consider species as abstraet (a-temporal) entities. This is a representative model only, which per se neglects the species-specific historical features.

${ }^{12}$ Species have a synchronic and diachronic dimension. The variation of its own members does not have consequences for the global unity $\operatorname{UG}(\mathrm{x})=\sum n \mathrm{X}$.

${ }^{13}$ Quine (1969) identified the word 'species' in a infra-specific degree of similarity within organisms. The debate over the natural kinds-terminology is really vast, see Schwartz 1981:301-302; Ghiselin 1981:269-282, Caplan 1981:130-140.

${ }^{14}$ Diversity is a qualitative variation within the members of a species. I utilise this word thinking of phenotypic characters. When we see physical differences between species specific individuals (colour of skin, somatic characters) we do not think that this diversity is also structural. Biological divcrsity within the members of a species respects those causal conditions that join species-specific individuals. We can thus say that a variation-limit exists in biology. Those individuals that go up to this boundary will be excluded from their parental species (since they physiologically cannot reproduce with the other member of their species) or they will dic from pathological mutations. This involves that a natural mechanism of mutants' selection exists, which ensures a progressive (not radical) change of species-specific individuals.

${ }^{15}$ Different units-of-evolution schemes have been defended by biologists through time. For example Hull (1980) called the taxonomic scheme of traits 'rcplicator', and the organised individual 'interactor'. Dawkins (1984) proposed two similar terms: 'replicator' and 'vehicle'. Despite having divergent theories about the biological organisation of individuals, both Dawkins and Hull agree that an organism's organisation acts as 'replicator of schemes (which engender lineages) as well as an interactor / vehicle' (Khalil 1997:199). This involves that the organism's organisation is self-organising and context-dependent. The interaction within an organism's constitucnts (and systems) is also oriented to its interaction with the environment.

16 Bradley Wilson analysed the problematic implicutions of the concept of species. He observed that: "(..) if species are individuals, then understanding how they can change through tine 
without losing their identity, how they can evolve, is no more problematic than understanding how other individuals, such as organisms, can do so" (Bradley Wilson, 1995:341)

${ }^{17}$ This is a basic condition. Even if we consider $U G(x)$ as $\sum n X$, we will need to admit a mininal causal relationship among $n X$ members. If $I$ am considering the canine species 'Alsatians' I will consider 'Alsatians' despite temporal or spatial borders. This involves that I need to find a causal link (functional or gualitative) within the nembers of a species.

"The species concept is not univocal. For example, botanists underlined the existence of difficrent kinds of species in vegetables: sec Cain 1954; Camp. \& Gill 1943: 323-385; Strebbins 1982:21.39.

${ }^{14}$ In 1976. Dawkins published his best sellar The Selfish Gene. This book popularised the growing view in biokogy that natural sclestion proceeds not in the interest of the species or of the group, nor even of the individual, but in the interest of the genes. The genes are the true replicators and it is their competition that drives the evolution of biological design. See Blackmore 1996, Dawkins 1976, 1996

${ }^{21}$ 1 will use the example of the human species because it is easicr to wonder about our own existence. My remarks can also apply to other animal species. The only problem is that when we talk sbout the human species we have shared knowledge that makes us aware to appertain to the global entity human race. If we think of different animal species, we will nol have access to their behavioural programs 'from inside' - for example - or we ought to bave specific ethological knowledge. Since 1 do not want to toeus ny analysis on some special animal forms, I have chosen to use the example of human species for its farniliarity. Clearly, my remarks can be extended to other animal species.

"Sociobiology allows us to clarify this step. It derives from Darwinian evolutionary theory. Witson (1975) hypothesised a strict relationship between genetic patrimony and social behaviours. He said that human and animal social behaviours could be explained by considering their genetic patrimony. This view is highly controversial.

${ }^{22} 1$ will stress the causal role or culure in the cohesiveness of individual-specific (and speciesspecifie) organic units in the following chapters of this dissertation.

3) "The gene is an instruction for buthing proteins, stored in a coll and passed on by reproduction. The meme is an insinuction for producing behaviour, stored in a brain and passed on to other trains by initation. However, there are many differences in the way the replication takes place. Cienes use the cellular machinery to copy themselves rather accurately. Memes have to be eopied by using the brain itself as the replicating machinery. This the also means that nemetic transmission is in sume sense Lamarckian. We can now see the human mind as the creation of two replicators, one using the machinery created by the other for its replication. As Dennett pointed out, people are animals infested with nemes. Our personulities, abilities and unique qualities derive from the complex incerplay of these replicalors. What then of our innermots solves - the "real me, the person who experieness 'my' life?" See Dennetl 1995. 


\section{I.4. The Organisms Environment Dialogue and the Organic Unity Theory}

In this dissertation I have defined the identity of an organism (and its species) as an organic unit, I now need to compare this view against other theories of biological evolution. The focal point of this chapter is the relationship between species-continuity and biological evolution. I will analyse the relationship between organisms and their environment at different levels of biological complexity. I will present reasons that will urge us to refute the view that biological evolution can be explained via molecular evolution. My analysis, however, on concepts of the individual in biology and my criticisms of mechanistic biology, do not aim at denying the physicochemical mechanisms in gene expression. I agree that a direct link between the physical features of living matter and the organism exist. What 'value' should be attributed to this relation?

Earlier I argued that genetic endowment does not have a basic value in biological phenomena since organisms are functionally integrated unitary systems of heterogeneous constituents, and the individual-specific gene pool's expression is a variable mechanism (gene plasticity) influenced by the genotype-phenotype relation. This mechanism is conditioned by environmental factors so that we can consider organisms as composite entities that maintain a cohesive coherence in their continuity. This is similar to the way that the biological existence of an organism adapts itself to environmental mutability in order to achieve fitness aims. I will argue this plasticity is a property of living matter at different levels of integrated complexity.

Modern evolutionary biologists (Provine 1986, Futuyama 1982, Willis \& Orr 1993) have recognised two contrasting twentieth century views on biological evolution and it's genetic basis: 1) Fisher's theory of natural selection" (Fisher 1930); 2) Wright's view of universal pleiotropy and evolution on "multiple adaptive peaks" (Wright, 1931, 1980).

Scientists are still debating over the problems implicit in both approaches. For example, quantitative genetics and statistical descriptions of the evolution of phenotypes, are still proposing different (linear or non-linear) models for use in biology (Aking 1983, Altenberg 1991). This problem is far from be solved (Pigliucci \& Schlicting, 1997:143-160). However, it is not my intention here to analyse the implications of quantitative genetics. I 
will use well known genetic concepts in order to emphasise my views, and to consider species as organic units and their evolution as a plastic process influenced by environmental factors and addressed to fitness finalities.

We know that evolutionary phenomena refer to genetic mutations and their consolidations in populations. These mutations aim at creating useful genetic variations that increase the phenetic fitness value. Natural selection favours particular sets of mutants that present the best fitness and reproductive capabilities. This mechanism seems unproblematic. If, however, we study evolutionary problems there are numerous obscure steps that need to be explained. Is evolution a chemical process of complex molecular changes, or is it based on the relationship between organisms and their environment? Is evolution explained by genetics or is it connected to phenotypes?

\subsection{Biological Paradigms in Evolutionary Microbiology}

Let us continue by analysing strong reductionistic biological paradigms. The common idea shared by these theories is that molecular evolution (population genetics) explains biological evolution ${ }^{3}$. Population genetics is concerned with the contributions of individual genes to the genetic foundation of an interbreeding population within a species, and the genetic makeup of a population over time. (Suzuky, 1988, p.95).

One example of this paradigm is found in Monod's Chance and Necessity. He thought of evolution as the result of minor independent genetic mutations that accumulated in populations and fortuitously recombined by genetic flows (Monod 1967). He stated that this phenomenon is statistically irreversible, and intrinsically oriented to the second law of thermodynamics. One must renember that, according to Monod, while the concept of species refers to stereo-chemical information shared by species-specific individuals (cti), evolutionary processes are subordinated to chance ${ }^{4}$ and necessity. The former is genetic mutation. The latter a teleonomic process that allows the continuity of species-specific genetic identity. Monod attempts to provide a clear example of mechanistic biology: natural evolution is explained by population genetics and species-identity refers to genetic similarity.

"In eflect natural selection operates upon the products of chance and can leed nowhere else; but it operates in a domain of very demanding conditions, and from this domain chance is barred" (Monod 1971:118) 
This view has influenced modern biology. In 1988, the Nobel Prize laureate Eigen wrote that evolution refers to population genetics. Eigen's central idea was that molecular changes are organised by thermodynamic mechanisms in population levels. The object of evolutionary selection was not the single organism but the spectrum of a population's mutants (the 'quasi-species' (heory).

Eigen defined the incidence of these mutants in the global population genetic equilibrium as their 'specific weight' in evolutionary mechanisms (Eigen 1975).

Eigen's view failed however to respect two concepts of 'classical' evolutionary theory ${ }^{5}$ : (1) Population-dimensions influence natural evolution and, (2) chance strongly influences natural evolution. According to Eigen, the first concept does not clarify the influence of an individual mutant toward its species. This incoherence caused him to hypothesise that, in Nature, neutral mutants exist in order to plant positive characters in their species-specific gene pool. Darwin's second hypothesis stated that evolution is addressed to favour individuals that have the greatest capacity for adaptation. Eigen discerned that experimental evidence does not confirm this idea. He proposed a species model in which evolutionary stages are preprogrammed at a population genetic level to consolidate adaptive mulations at a population level. Chance, necessarily, would remain an element influencing evolutionary dynamics. This is conditioned by two additional factors: quasi-species and genetic systems' physical tendency to have equilibrium-conditions ${ }^{6}$.

The above conclusions are based on Eigen's computational models (fractal systems). Computer simulations and the composite nature of populations impelled Nagel to use statistical tools to evaluate the consolidation mechanisms of particular characters within certain populations. This mathematical approach pushed the German biologist to emphasise that at a 'macro-state' complex systems tend to equilibrium '. This means that evolutionary genetics, Nagel says, can be analysed by stressing micromacro state relations in population levels, (such as mutations and their consolidation in a species). Evolution is then tightly linked to individual mutation distributions (microstates) within the species level (macro-states).

Another example of this use of statistics in population genetics is provided by Kimura's theory. In 1983, Kimura discovered that genotypic and phenotypic evolution operate at different speeds. The rapidity of genotypic evolution is less than the speed of species-specific phenetic evolution. He 
observed that a proteins' polyformic structures are equivalent in an evolutionary perspective (synonymous sequences). This result impelled him to claim the thesis that molecular evolution is influenced by 'blind' mutations. The affirmation of a certain protein then relies upon fortuitous elements (Kimura 1983). Molecular evolution is a process based on two processes, natural selection and fortuitous genetic drift ${ }^{8}$. All these views are similar in that evolution is considered a natural phenomenon explained by physicochemical mechanisms. Monod stressed the teleonomy-invariance relation, and Eigen emphasised stochastic mechanisms, while Gould explained evolution via a punctuated mutation mechanism (a mechanism of unexpected acceleration in the mutational phenomena ${ }^{9}$. All proposed a mechanistic approach to evolutionary biology. Can we accept this approach to explain natural evolution?

The genome has a fundamental role in evolutionary phenomena. It is both container and vector of the mutational mechanism. However, if we consider DNA as the fundamental agent of evolution, we think of the individual-specific (species-specific) identity as a genetic element. In doing this, we completely distort the biological concept of population; the population as a whole of individuals possessing an individual specific biodiversity. Why should mechanists deny the composite nature of biological individuals? In my opinion, the conceptual reasoning used to defend the mechanist's view is a general assumption that a species is a whole of numerical units, a numerical class. In this sense, an organism's individuality becomes a mathematical singularity ${ }^{10}$.

Simply, if a species is an identity because of genetic characters shared by its constituents, then the individual specific individuality becomes its difference with regard to a species-specific genome. However, not all members of a species may have all the diagnostic traits that tell them apart from similar species. Species that share all the same diagnostic traits are a rarity and are labelled 'monotic' species. Species usually share only some diagnostic traits among all members. These members are labelled 'polytypic' species. Polytypic species constitute the majority of living species in nature. This involves that the species concept is based on diversity anong individuals rather than similarity among individuals. An emphasis on this element individuates a species-specific property but does not explain the meaning of natural kinds. I object to the view that biological uniqueness is not a mathematical property. In fact, mathematical uniqueness does not explain the individual specific (or species specific) identity on its 
own. Let us think of our own existence. If Pasquale and I are both in a room, then the room contains two humans. Neither the identity of Pasquale or myself is contained in the term two humans. If we consider the people in the following figure, we have a description of their spatial presence. When we use this model to represent their identities, we translate their 'individuality' into a formal system of representation. Their identity could also be symbolised by numbers or chemical formulas. In doing this, we use another representative system to symbolise these individuals. Can we consider symbolic models as systems containing objects' features? Symbols say nothing about an object's existence, they simply represent it. Similarly, the translation of biological diversities into mathematical diversities symbolically represents the individuality of organisms. We do this to achieve a specific goal (for example, to calculate molecular mutation rates of a specitic protein) and to draw conclusions from the representative system. It would give us information concerning some peculiarity of the analysed object. This does not mean that objects' existence is explained in our classification.

Symbolisation of a whole of human beings.

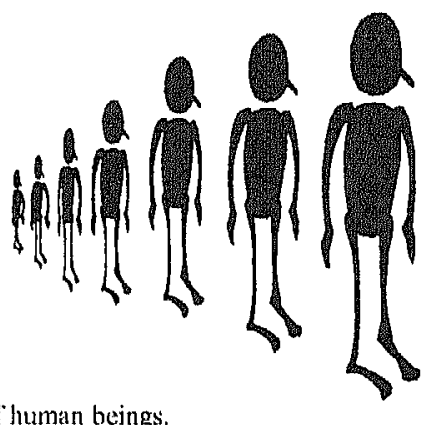

Nor does it mean that our representation contains all the peculiarities of an object. Following this, we might think of biological diversity as an insignificant element in a species, which would be erroneous as we would base the concept of species on species-specific genetic homogeneity (cti). This element would contain the species-specific essence and would be substantially stable over a period of time. The genome, thus, would be all we need to know concerning the organism's (or species') biological existence. In extensive proportions, the physical (stochastic) variations of 
this element would explain the evolutionary changes (think of Eigen and Monod's theory).

Consider the human species. Would you say that it is the sum of six billion (more or less) humans living in 1999 on Earth? Even if the human species respects the conditions to belong to a specific species, the knowledge of these properties alone does not explain the socio-historical nature of human beings. This historical nature portrays the potential consequences of any attempt to translate an individual's biological singularity into numerical systems. We might accept the relation 'single organism = one number' if we thought of individuals in abstract terms (for example, statistical data of the number of milk drinkers in Rome). This abstract classification would not explain the peculiarities of single organisms. It is incapable of analysing the meaning of an organism (or species) as itself, so we use a monodimensional representation system in order to translate the multidimensionality of natural kinds. This system is an abstract and arbitrary tool fixing a plastic-dynamic-heterogeneous object in an a-temporal and inmutable representation.

The possibility of a mono-dimensional representation system encourages us to stress constitutive similarities, not qualitative diversities, amongst symbolised entities. This is because numerical systems in themselves cannot represent the qualitative diversities among individuals. Mechanists thought of these biological variables as statistical or probabilistic manifestations of evolutionary non-linear dynamics in complex systems, such as populations and species. The constitutive property of living systems is their composite and heterogeneous nature. Likewise, the explanation of biological phenomenon cannot be explained by a theory of reduction, because the interaction of local occasions, be it physical, organic or ecological phenomena, constitutes the history of an organism. Simpson suggests that we are to explain evolutionary directions as global historical occasions (Simpson 1967). Mechanists cannot accept this view, as too many variables are involved in the natural phenomena, too many stochastic laws! How do we explain individual humans via statistical and reductionist tools? How do we explain the uniqueness of a single organism via stochastic recurrences?

\subsection{Mathematical Models in Biology}

I mentioned a few generalised criticisms concerning the use of mathematics in biology. Let us analyse some practical examples of systematic biology and the differences between physical and biological 
systems. When we talk about living beings are we talking about physical systems as formed by atoms, electrons and quanta? What difference exists between organic and inorganic systems?

Organic systems have specific features, such as their homeostatic nature, self-replicating capacity and energetic exchanges with the environment. They are "characterised by a remarkable complex organisation which endows them with the capacity to respond to external stimuli, to bind or release energy (metabolism), to grow, to differentiate, and to replicate. Biological systems have the further remarkable property that they are open systems, which maintain a steady-state balance in spit of much input and output" (Mayr, 1981:14). A major difference between organic and inorganic systems is the magnitude of complexity of organic matter. For example, DNA contains billions of deoxyribonucleic bases, which duplicate continuously. This complexity obstructs any attempt to individuate stochastic (or algorithmic) recurrences in biological phenomena. However, this does not clarify the qualitative differences between physical and biological systems. If this difference was only a difference of complexity, it could be possible that the increase in computational systems would allow the use of 'physical' science to analyse organic systems. Cohen (1969:217231) focused his researches on this problem. He pointed out that biological systems are not simply complex physical systems, but that they have further properties on their own: (1) they are coherent systems (2) they are selfreorganising and, (3) they can reproduce consistently stable functions. The first property can be explained by considering the organic unity concept. The organisms are cohesive units of heterogeneous sub-diversities. This unity-in-diversity continues through time despite its variations. The continuity-in-Variability of biological entities defines the coherence of biological phenomena. Why, via mathematical systems, can we not symbolise this coherence? We could think of the reproduction of complex functions as the capacity of a system to reduce numerous parameters to a condition of stasis. For instance, cyclic mechanisms could reduce a variable complexity to a whole of constants based on simple and repetitive mathematical dynamics (algorithms - Hacken 1978; Eigen and Schuster 1978). We could presumably do this by assuming systems are cyclically organised by mono-parametric dynamics. Then we could use mathematical tools (Hacken's principle, for example) to explain the cyclical organisation of complex systems. This system cannot ever truly describe the interaction among heterogeneous systems, and therefore, cannot describe biological phenomena. This involves that when we describe two systems interacting in 
a bipolar phenomenon, we cannot predict how their interaction will characterise the observed phenomenon (Galzaniga and Muir 1988:151-168). We also cannot predict the status of non-linear systems, because they are the result of different, and synergetic, causal elements. Since biological phenomena are the results of synergetic causes, the use of mathematics in biology is limited. (Note that 'limited' does not mean that mathematical models are useless in biology. Their use in biology has to be considered functional (and not exhaustive) to explain the organic systems' features.)

Consider now the practical example of quantitative genetics. In a recent paper Pigliucci and Schlicting summarised the assumptions of genetic models as follows:

\footnotetext{
(i) Lincarity. Genetics and environmental elfects on phenotypes are expected to be described. (...) (ii) Additivity. The assumption of additively amounts to the relegation of any interaction - among genes (epistasis) or between genes and environments (GxE)- to a minor role. (...) (iii) Normality. The description of invariate or multivariate (Lande 1979 , Lande \& Arnold 1983) evolution is easily accomplished if the genetic effects follow a (multi-) normal distribution, or if selection is extremely weak. (...) (vi) Constant of the genetic variance-covariance matrix. The matrix describing the genetic variances and covarianes between dillerent traits $(G)$ is fundamental to predictions of evolutionary trajectorics." (Pigliucci \& Schlicting 1997:146-147)
}

The central idea of quantitative genetics is the possibility of creating a functional model by which we can explain (via a statistical summarisation) the organisms' phenotypic traits, and predict their evolution via mathematical models. The problem arises: Is it really possible to explain the individual features via mathematical models? If we can answer in the positive to the first question, then another appears: What type of biological system should we numerically symbolise?

The first problem is easily solved. We can summarise the individual's features, but only in a limited sense. The variable (constitutive-qualitative) nature of an organism's constituents cannot be mathematically summarised. We can partially describe an individual's traits, but the magnitude and complexity of data, and their continuous change, will not allow us to completely explain the features of an individual by mathematical formulas (Lewontin 1999). We can focus our description on some traits of its biological existence (e. its genetic structure) but this process is limited. The problem becomes more difficult when we try to attribute a basic value to our descriptive system. Numerous authors have discussed how quantitative models do not completely explain organisms' features or biological phenomena (such as phenotypic evolution) (Lewontin 1982, Pigliucci 
\&Schlicting 1997, 1995). When we try to extend them to other biological problems, numerous implications come into play which limit the exhaustive value of mathematical models in biology. Subsequent examples have been phenotypic expression of genes, gene-environment relations, and evolution in changing landscapes (Pigliucci and Schlicting 1997).

It is questionable whether quantitative models completely address explanations of natural phenomena. Have these extensions relied on an incorrect usage of this discipline? I personally believe that this use of mathematical models does not pertain to quantitative biology. It seems that an incorrect use of science per se obtains incredible results in the exploration of organic systems' features. Maybe we should use mathematical models, in a limited sense, as a heuristic tool for analysing natural phenomena, but certainly not as the explanation of their meanings.

The second problem involved in quantitative biology refers to the model we choose to use. If we think of mathematics as a deductive system based on initial axiomatic premises, its use in biology refers to the existence of a universal model, one that could explain the relationships between investigated phenomenon and their translation into mathematical systems. Clearly the complex and synergetic nature of biological phenomena make the choice of using mathematical models in biology very difficult. The synergy of biological phenomena persuades us to link these mathematical analyses to other physical constants (Arhenius' constant, or Boltzmann's constant). As biological phenomena are the result of numerous determinants (Ageno 1986), its actuation does not necessarily respect the expected physical results. Quantitative biology needs to use mathematical models that take into account the possibility of non-linear evolution of the analysed systems.

This non-linear evolution of biological systems has convinced biologists to apply non-linear mathematical models to biology. The central mechanism of this process refers to the mathematical translation of biological or physical elements within an analysed phenomenon (for example the distribution of specific gene traits at a population level). After this symbolisation is done, deterministic equations are used for analysing the evolution of the symbolised systems. (Non-linear models are also used in other fields of science e.g. physics and ecology (Hastings 1994). Another extension from non-linear dynamics is complexity theory ${ }^{11}$.) The choice of an appropriate mathematical model in biology is controversial and difficult. Non-linear models, such as algorithms, translate the organism's traits into a symbolic system. This symbolisation mechanism is also problematic, since it 
translates a variable entity, the organism, into a deterministic system. Even if the translated entities are subsequently analysed via non-linear mathematical models, these last ones cannot be considered exhaustive. They symbolise individuals (or they symbolise traits of individuals), but this symbolisation does not envision the nature of living beings. As the translation of organic systems into numerical systems is problematic because of the living being's heterogeneity, the synergy of causal elements in biological phenomena limits their explanation through physical mechanisms. The dependency of a biological phenomenon on its context limits the use of mathematics in an extensive explanatory biological model.

Since living matter has physical features, we can use mathematics to analyse the mechanisms of these phenomena, always remembering that those features cannot be completely explained via mathematical models. We have to base our analysis also upon a set of qualitative properties of living systems such as environmental relationships, behavioural programs, mental states, and the synergetic interaction of living systems towards biological phenomena in organisms.

Then, the description of living systems via mathematics is only an indicative explanatory tool. If we were only interested in the meaning of biological phenomena, the use of mathematics would be of little help, since it is always limited by its symbolic value (it can describe, but a descriptive model is not an explanation of a phenomenon, Salvi 1996). According to this, quantitative biology can help in providing theoretical synthesis, such as in my analysis of the concept of individual, and in offering data over the (short-term) evolution of peripheral biological phenomena. These models are extremely useful in themselves, but they cannot be arbitrary expanded to investigate other areas. Their use is functional in the way they explain natural phenomena. The explanation of complex biological puzzles needs further comparative analyses.

\subsection{Dialoguing with Nature}

Francois Jacob (1982) stated that it is impossible to explain evolutionary phenomena through DNA changes. Jacob proposed that genetic mutations can be accumulated in modified proteic structures constituting of a container of possible proteic variations. These proteic alternatives allow positive new environmental adaptations. Likewise, selective pressure perturbs the interaction mechanisms among proteins. Thus, the natural evolution allows for proteic variations. In considering 
these biological mechanisms, Jacob concluded that the large number of species-specific gene pools is the result of a 'few ancestral genes' mutations. The incipit of molecular evolution might rely on simple bacteria possessing the fundamental bio-chemical features of life (e.g. selfreplicating capacities, metabolic functions, and mechanisms of energy control).

Evolution, in Jacob's view, does not begin by biochemical innovations in different organisms, but by the inverse process. This means that environmental change and selective pressure cause biological variations, and not vice versa. The meaning of the 'colloquial relationship' between organisms and environment then becomes central. Is the dialogue between organism and environment the cause of living beings' molecular constitution? We will analyse this question in the following pages.

Mayr writes that evolution is not a genetic mechanism but that it refers to the organism as a 'whole' (Mayr 1988:101). The organism's identity thus cannot be explained by its genome. It relies instead on the co-presence of different biologically integrated heterogeneous systems. According to Mayr, biological evolution has a coherent meaning only when it is analysed at the population level, since we cannot explain evolutionary mechanisms through the chemical variability of isolated genes, or genetic pools (Ayala and Dobzhansky 1974, Strebbins 1969, D'archy Wentword 1942). The phenetic evolution has a basic role in evolutionary processes because the unity of selection is simultaneously 'the individual', and 'the diversity among species-specific individuals'. A species is a morphological unit because it is a unity-in-diversity. Its unitary meaning refers to casual factors of cohesiveness, the 'c-condition', mechanisms of similarity among the members of a species, and the genetic flow. DNA is an important factor of cohesion among members of a population, yet it cannot be considered as a singular evolutionary causal mechanism.

In the preceding chapter, I have stressed the causal r-relation within members of a species (their reproduction clause). In parallel, I have stated that species continuity refers to a flow of genetic information. The genetic flow is a vector of genetic (shared) information within members of a population, and contains encoded information that will be inherited by descendants. We need to question whether the role of genome(s) in population biology allows us to explain natural evolution by genetic evolution at population levels?

Both Jacob and Mayr do not attribute to DNA this basic value in natural evolution. Jacob stresses the impossibility of explaining the processes via 
chemical changes, and considers structures of an organism as being in flux. Mayr considers species as the unity of selection. According to this view, biological evolution refers to genetic vectors (genetic flow), but a speciesspecific genome is not the subject of evolution as evolution refers to the global entity 'the individual as a whole'. This means that evolutionary mechanisms refer to genetic consolidations of specific gene recurrences in population levels, although evolution is not explained by the genetic frequency's change in populations. Genes are only a vector and container of evolutionary change. Selection is linked to phenetic selection on a species level.

According to Prigogine's theory, living matter continuously increases its own complexity. Likewise, the degree of heterogeneous and cohesive complexity increases in the organic-unity species. This integratedheterogeneity relies upon the organism's complexity. This means that the complexity, the degree of members of a species, is extended to the complexity-degree of the species on its own. The degree of unification and the number of unified sub-constituents are directly reflected in the global degree of complexity of the organic unity species. A use for the organic unity concept in systematic biology would be to defend biological taxonomies based on the structural/qualitative biological complexity of individuals ${ }^{12}$.

In parallel, the physical laws according to which simple structures evolve into complex ones explains the evolutionary growth of species-specific structural complexity, and how it refers to the dialogue between organism and environment. We can recognise different mechanisms of dialogue between environment and organisms. At an elementary level (a bacterium) the dialogue is oriented to reproduction. But a complex organism has numerous factors co-participating to this process:

Table 1.3

(a)Internal elements of Organic Unity (b) External elements of Organic Unity

- homeostatic processes fitness to environment

- metabolic processes dialogue with environment

- regulatory processes - social bchaviour

- central nervous system - linguistic capacities

- awareness and/or self-awareness $\quad$ culture

When the complexity of an organism (and a species) varies, its degree of organic unity changes. The co-presence of unifying factors increases the 
degree of unity-diversity. Since organic systems are composite and heterogeneous dimensions, the causal r-relation joining their constituents is not univocal. Depending on the species, the modality of this mechanism will vary, but its meaning remains the same:

An organism (and a species) is a heterogeneous complexity joined in a unitary dimension. This unity-in- (variable) complexity is based on linked mechanisms that ensure its continuing existence. The existence process refers to the r-relations that connect the organism (and species) constituents. The above mentioned mechanism links organic systems to their environment. The heterogeneity of life forms admits the possible existence of numerous $r$ factors keeping a functional dialogue with the environment. This mechanism belongs either to single organisms or to their species, because both are composite structures. In parallel, the clustering factors do not refer only to biological elements but also non biological mechanisms, such us cultural phenomena, instinctual behaviour, sociobiological mechanisms, etc. This involves that:

Accepting the organic unity view means moving the heart of the biological identity problem from the individual-specific biological requirements (this implies the quarrel between reductionists and nonreductionists) to the r-relation, which makes the individuals or the species a coherent and unified, heterogeneous and variable structure.

Biological identities are open systems that converse with the environment, and transform themselves while remaining homogenous entities, and organic units. The analysis of their features refers to their composite and variable nature. Then, the central element of the species' (or organisms') existence becomes the mechanism of coherence and cohesion within its life stages.

\section{NOTES}

\footnotetext{
${ }^{1}$ Fisher claims that: "phenotypes [are] determined mostly by polygenic inheritance, with many loci characterised by small and additive effects controlling cach trait, evolution would happen gradually, primarily in large populations, ... and would eventually lead to a single equilibrium representing the state of maximum fitness" (Pigliucci \& Schlicting, 1997: 143-144).

${ }^{2}$ Wright said that: "phenotypes are controlled by complex interactions between genes and ... evolution [is] happening mostly in small and peripheral populations, subject to rapid accelerations and constant turmoil, capable of moving toward multiple adaptive peaks in a complex and ever-changing litness landscape" (ibid. p. 144).
} 
${ }^{3}$ The impossibility of predicting the incidence of genetically induced epigenetic mutations in natural evolution is still being debated in biology. In a recent paper Vines criticised genctic reductionist chaims that genetic inheritance plays an exhaustive role in individuals' life (their phenotypic features as well as their cualitative expressions). Dr Vines stressed the impossibility of reducing inheritance to chemical processes. Organisms' epigenesis shows strict links between individuals and their environment. (Vines 1998:26-30; Reik and Surani 1997; Jablonka and Lamb 1995; Roemor 1997:277)

4 "We call these events [molecular changes] accidental; we say that they are random occurrences. And since they constitute the only possible source for modifications in the genetics text, itsclf the sole repository of the organism's hereditary structures, it neecssarily follows that chance alone is at the source of every innovation, of all creation in the biosphere. Pure chance absolutely free but blind, at the very root of the stupendous edifice of evolution." Monod, 1971:112

"The three essential principles of Darwin's thcory: 1) "The principle of variation: In any natural population of organisms, individuals can be expected to differ from another in a variety of ways -structurally, functionally and behaviourally. 2) The principle of heredity: Members of the same species are linked by bonds of biological inheritance, which tend to transmit many of their characteristics to their offspring. 3) The principle of selection: Annong this assortment of inherited traits, some inevitably contribute more to the survival or reproductive success of an organism than others." (Lewontin's definition (1970) quoted in Suzuky, 1987: 96)

"The orientation of this process is not verified in only one direction at the micro-state level. Equilibrium for each micro-state is not defined. We can define the "state of equilibrium" loosely as the "memory" of micro-state individual, independently to its macro-state ." Eigen, 1975:185 [mine the translation]

'The problem of thermodynamic equilibrium is really important because biological phenomena (at the molecular level) are linked to chemical and physical laws and thermodynamic dynamics cause mutational processes.

${ }^{*}$ Gould proposed another view (punctuated equilibrium theory). He said that natural evolution has accelerations (punctuations) alternated hy long phases of stasis. Given this, evolution becomes the phenolypic translation of these macro-mutations.

"Punctuated mutation entails that traits are not incidental, (c.g. arising from stochastic frequencies). The central premise of Gould and Eldredge (1972) was that evolutionary chango alternated long periods of stasis with brief periods of rapid change (punctuations). According to this, a gradual evolution does not exist, since evolutionary change proceeds by jerks. "Speciation is not always an extension of gradual, adaptive allelic substitution to greater effect, hut may represent (...) a different style of genetic change - rapid organisation of the genome, perhaps non-adaptive" (Gould, 1980:119). The punctuated equilibrium theory has been strongly criticized by other biologists, such as Dawkins (1986), Sterelny (1992). An analysis of this querelle is Dennett (1995:283-299). Biologists have identified a genetic transcription unit designated Sdic (sperm-specific dynein intermediate chain), which might influence molecular evolution. This suggests that evolution could have rapid acceleration influenced by genetic factors. Sec Nurminsky et al. 1998:572574, Begun et al. 1992:519-520

${ }^{10}$ Take two organisms $X$ and $Y$. If $X$ 's identity is $\sum$ DNA features - $P(x g)$, and $Y$ 's identity is $\sum P(y g)$, it follows that $X$ and $Y$ both pertain to the same population if they contorm to the 'species specitic genome model' [we can consider this element as follows: $\sum \mathrm{P}(\mathrm{xg}), \mathrm{P}(\mathrm{yg})$, $\mathrm{P}(\mathrm{zg}) \ldots . . \mathrm{nP}(\mathrm{g})$ ]. Following this dynamic, a species is a set of orginisns that has structural 
genetic similarities. The differences between $X$ and $Y(\Delta X-Y)$, is: $\sum[P(x g)-P(y g)]$. This means that infra-species differences become the quantitative variation between heterogeneous DNA joined by a species-specific physico-chemical similarity. Thus, the biological diversity would be explained by mathematical (or statistical) data, because individuals are considered numerical units whose sum defines their species.

11 This theory has numerous biological applications: the study of the evolution of complex genetic algorithms (Neumann 1966, Eigen 1975), phylogeny of cellular automata (Nijhou 1986), and genetic networks (Kauftman 1992). A deeper analysis of this problem, and other areas of application for quantitative biology is Pigliueci and Schlicting(1997:153-4.) Other applications of non-linear models are Game Theory and Optimisation Theory (Eigen 1975), and Chaos Theory (Lewin 1992).

${ }_{12} \mathrm{~A}$ bacterium has a minor degree of complexity compared to a mammal, because the latter possesses a higher structurally integrated complexity in its physiology. Since a species is a composite entity, the increase of complexity of an indjvidual will be extended to the species degree of complexity. This view can explain why human species have a superior degree of organic unity in natural world. Since the clustering factors unifying human species arc numerous (cultural, social, artistic, tcchnological, creative, and dynamic elements) they each add to the degree of complexity of the human species. In this sensc, we can defend a hierarchical relation amongst animal species. This taxonomy would be based on biological (and not ethical) factors. 


\subsection{Neural physiology and Self-Synthesis.}

In the first section of this dissertation, I stated that an organism is an integrated system of heterogeneous constituents. Such an integrated system of heterogeneous constituents is also found in the analysis of the brain's biological features. Using our knowledge of the structure of the organism, we can use this 'analogy' to gain entrance into the structural organisation of nervous system. (This is an incredibly complex process as the human brain cortex has on average 10 billion neurones and 1 million neural connections).

In this chapter, I will study the value of mental phenomena with regard to the existence of organism as a specific individual. I will analyse different perspectives involved in this topic. I will study the philosophical, psychological, and biological problems involved in individual's selfsynthesis. This is because an individual consists of an embodied psychology, which incorporates the relationship between the organism and its experiences.

We will see that the organic unity theory can be successfully used to explain mental phenomena such as self-synthesis, menory, self-awareness, etc. Biological explanations of Mind can help in providing a better understanding of the nature of individuals because, as I shall explain, mental phenomena are inseparable from the mechanism of life regulation and play an inherent role in the biological processes of Life. Let me begin by considering some neuro physiological results that encourage us to accept this view.

\subsection{How does the Brain Work?}

Kofka's experiments on perceptual phenomena showed the active role of subjects in their perceptions (Kofka 1935). According to his views', a subject organises its sensorial stimulus in 5 different ways: proximity, continuity, resemblance, symmetry and closure. For example, Gestalt mechanisms act as the unconscious structuring processes behind our everyday perceptions. In opposition to standard views, rapid movements of the ocular globe are central in constructing our observations (Darley 1986, Pinker 1984). A subject organises its perceptions through determined neuro physiological mechanisms, its approach to reality being conditioned by 
biological mechanisms that regulate its perceptual capacities. How does the central nervous system actually perform this work?

In 1980, Donald Hebb proposed a theory that conceived of the brain as a 'self-organising' structure. He suggested that the cerebral system is composed of interconnected sub-systems that are organised in closed correlated sub-units (cellular wholes). These 'wholes' are excited by internal stimulus that are not necessarily caused by external sensorial inputs (reentrant mechanisms). The brain structure thus is organised in cellular units forming circuits of neural transmissions. The cellular wholes are a consequence of environmental inputs (sensorial inputs) and form neural circuits of mental phenomena (visions, perceptions, ideas, emotions and so on). In this sense, the individual neural physiological existence refers to the sensorial inputs that a subject has during histher life-stage (particularly during his ther cognitive development).

Hebb asserted that because the physiology of the brain is both structurally and qualitatively conditioned by perceptual phenomena, cerebral neural physiology and neural topography are intrinsically connoted in terms of uniqueness.' This means that the brain's topography is influenced by environmental (perceptual) inputs, and that the brain's cohesiveness refers to mechanisms of interaction among cellular units ${ }^{2}$.

A number of different experiments (Baringa 1999) provide evidence of the causal role of experiential acts in the formation of neural circuits ${ }^{3}$. The spatial configuration of neural-circuits is the structure through which the perceptual inputs are introduced, transmitted, and eventually, elaborated. The experiential phenomena thus 'cause' the neural circuits, and influence the interconnection mechanisms that reside within brain units. According to this, the relationship between an organism and its environment influences the structural constitution of the brain, as well as mental phenomena. This process individualises the organism's structure. This indicates that organisms link their heterogeneity via a flexible (plastic) relationship with the environment. In the central nervous system, perceptual input allows an organism to claim structural and psychological singleness. In parallel, biological mechanisms allow the brain to continuously adapt to environmental variables, i.e. through feed back mechanisms and the interconnection between neurones and within neural cellular units.

The buman brain itself is an excellent example of such integrated complexities. The human brain can be divided into two different systems: 1) The encephalic trunk and the limbic system; 2) The thalamus and cortex 
(Shepherd 1983, Changeux 1986). The first structure does not receive direct sensorial stimulus. Its primary function is to regulate the organism's physiology (homeostatic function). The second structure is more complex. It regulates the motor behaviour, psychological phenomena (memory, primary and superior consciousness, intellectual phenomena), the reception of sensorial inputs and their reply and is characterised by neural links and quick responses. Cortical cells are organised in microcircuits (fibres) and composite cellular units (neural maps) strictly correlated by feedback mechanisms. When an experiential stimulus arrives at a neural map, it will process and transmit the stimuli to the other neural maps. At the same time, it receives signals in response from previously transmitted stimuli. This means that neurones of different maps can be simultaneously excited by, elaborating on, and transmitting, the same experienced stimulus. Feedback mechanisms play their role by increasing the correlation of neural phenomena. Differing sensorial stimuli cause different topographical links amongst groups of neurones, with different inputs determining various structural changes in the global topography of the brain.

Each organism, then, is a self-organising system (Edelman 1992) that has a hierarchically organised series of feedback processes contained in the individual's nervous system. This mechanism is enriched by another system of physiological re-organisation (the endocrine or regulatory system) by which organisms react to environmental stimuli (transmitted via the nervous system). Both these systems are mutually correlated ${ }^{4}$ (Edelman 1992, Ballain \& Dickinson 1996, van den Bos 1997). Organisms are qualitatively and structurally joined to their environment and can adapt themselves to environmental conditions. Input signals from outside the organism cause internal reaction signals that alter the individual's physiology, and evoke (cause) behaviour that functions in the survival of the organism. This involves that: 1)The brain is a heterogeneous structure which has a unitary meaning; 2) The relationship between the organism and its environment influences the brain's structure both topographically and functionally.

\subsection{Quantum Theory and Neural Interconnections}

So far I have emphasised the brain's biological features. Now I shall analyse another example of reductionistic biology; the use of physics as an explanatory tool in the field of biology. The central assumption of neural physics is that when we realise that our brain is constituted by cells, and these are the expression of a physical reality, it follows that the neural 
phenomena are subordinate to the laws of physics. The brain's basic constituents are linked to quantum phenomena at the level of neural connections (synapses). Since the dimension of synapses is $2 / 1000 \mathrm{~cm}$, we can consider a direct link between the physical factors of synapses (and thus, neurones) and quantum physics. This premise looks reasonable, but becomes problematic when we try to explain mental activity using physical explanations. The interpretation of the Copenhagen' School of quantum physics allows us to consider the use of quantum theory in studying the activity of neural structures (Penrose 1991, Lockwood 1989; Zohar 1990). The question arising is; 'Is physics the 'right' scientific tool to explain the meaning of organic phenomena?'

When we use physics as 'the' explanation of biological phenomena we must be aware that its epistemological nature does not appropriately translate the nature of organic phenomena. Physics, in fact, uses calculation and prediction to explain the general mechanisms of the natural world and their recurrence. It translates the individual objectivity of a phenomenon into a universal objectivity showing how the mechanism ex quo the phenomenon, is determined.

The conceptual framework of biological thought and investigation is completely different from that of the physical sciences, and cannot be reduced to it. Biology cannot use the law-concept of physical sciences, because biological phenomena are the result of synergetic causes. The composite nature of organisms emphasises their individual uniqueness, ${ }^{5}$ or defines the context-dependency of biological phenomena. 'Biological laws' are not the universal laws of classical physics, but simply "high-level generalisations" (Mayr, 1981, p. 19). If we try to use the tools of physics in biology to explain biological phenomena, then we have endless difficulties in fitting general laws to local regions of generalisation within biology. The predictive ends in physics can be achieved because deterministic laws regulate the actuation of phenomena (Newton's mechanics). In parallel, physical phenomena have constant parameters (mass, density, velocity, potential and kinetic energy, etc..), which ensure the possibility of perceiving accurate and useful results that can be handled in a 'predictive' fashion. But, organic systems do not act as inorganic systems.

In biology, the high number of interconnected phenomena (e.g. meiosis, gastrulation, immunity system, neural topography, and so on) make it difficult to achieve predictive results. In this sense, the pluralism of causations and solutions make prediction more probable. Both the complexity of biological events and the frequency of unexpected 
(unpredictable) phenomena (for example, genetic mutations) obstruct the possibility to individuate biological laws as recurrences. We can use them only if we continuously de-categorise them by underlining their probabilistic meaning ${ }^{6}$. Uniqueness, complexity, and unpredictability are properties of living matter which mediate against the use (or methods) of physics to explain living beings at the appropriate level.

In biology, we have to substitute the physical concept of law (deterministically driven), with another one (probabilistic driven) thinking of law as 'probability' (Mayr 1988, Ruse 1973; Hull 1974, Dawkins 1996); A biological phenomenon has constitutive peculiarities and cannot use physics to explain natural phenomena. This, however, does not mean that physics cannot be used in biology. The techniques of the hard sciences can obviously help explain living matter's structure and organisation, but this analysis requires further research (on behaviour, evolution, fitness, etc.) if it is to explore the 'meaning' of natural phenomena. (Prigogine and Stengers, 1984)

\subsection{Quantum Physics and Neuroscience}

Returning to the initial question of how quantum physics might explain the properties of the human brain, we can look at the limits of physics as they may apply to neuroscience. First, we need to understand what a neural phenomenon is. In analysing an experiential phenomenon (the taste of a apple), we can call 'mental activity' the electrical stimulus that is transmitted from taste buds to neurones. This neural connection, however, does not explain our feeling of taste. There are two properties of the above mentioned phenomenon: its physical nature and its qualitative nature. The first is the neural transmission, the second, the elaboration of neural data.

Neural physics analyses the physical mechanisms involved in experiential acts. If we consider the example of taste, we can register and measure the electrical activity of neurones involved in the procedure of actually tasting an apple (taste/apple transmission). We know that the perception of a taste is a cascade of chemo-physical events that result in an electrical impulse of a specific frequency, wave-length and intensity. This impulse is the physical translation of a taste. Can we say that the taste is explained by electric impulses?

This is an extremely problematic point. On the one hand, it is true that sensorial data are translated in physical elements (energy, electricity), but on the other hand it is also true that a taste is not limited to electric 
transmissions. In fact, even if sensorial inputs relate us to external reality, the mechanisms of neuro data-elaboration cannot be explained only via mechanic processes.

When I say that my perceptions are explained by my perceptual inputs, I deny my active agent-of-perception-role and I think of myself as a passive container of neurological data. This means that when perceptual inputs are transmitted to my brain, the mechanism of neural maps, 'feedback,' 'memory,' and 'attention,' describes the weight I attribute to them. My perception of sensorial stimulus therefore refers to myself as "experiential agent'.

The gap extending between data reception and subsequent elaboration can be investigated by neural physics. Nevertheless, when we explain our neural data 'interpreting-functions' (their 'attribution of value', the individual approach with reality), we need to link the physical explanations to biological explanations (from neural physiology, embryology, evolutionary biology, psychology and philosophy). Neural physics explains the chemicomechanical modalities of neural transmissions, but when we pass from the transmission of a sensorial input to its categorisation and elaboration in the brain it seems impossible to approach neural phenomena from the vantagepoint of physics alone. In fact, the formation of neural circuits and their interconnection are the neural physiological result of an initial neural transmission. Yet, when the received data are elaborated upon and compared with other neural maps (via feedback mechanisms), the mechanistic description of data in terms of electrical levels says nothing about their 'interpretation' in a brain. This is a constitutive limit of neural physics, since its cognitive tools are oriented to quantify a phenomenon and not to interpret its qualitative meaning.

If a portion of my cortex is stimulated by electrical stimulus having the correspondent wave-length of electrical stimulus received by a subject looking at a cat, I do not visualise the image of a cat. In fact, Hubel and Wiesel's experiments on vision mechanisms say that the localisation of neural circuits by which the visual inputs are transmitted to the brain, cannot explain the modality of their translation in a specific image (Hofstadter 1979:373-396). We can use neural physics to clarify how these inputs are transmitted, but we need to use different tools for understanding what these data 'mean' with respect to a particular subject. Physics allows us to clarify the brain's functions at its micro-level (neurones), but when we try to explain the mind's peculiarities at the global level, we need to address 
omplex neural phenomena on the level of complexity seen in the primary nd secondary consciousness phenomena.

\section{Is Neural physics Really Interested in Explaining Consciousness?}

When neuroscientists say that mental states can be explored arough quantum physics or chemistry, they are presuming a quality that is erhaps absent in physics or chemistry. For example, those who say that hysics will provide an explanation of vision processes, they distort the role $f$ subjects in experiencing reality. When 'vision' is an experiential act 3 volving an experienced object and an agent of perception who approaches sality in a 'subjective' way, the subjectivity of that experiential agent ecomes central. We superimpose the perception onto a neural data system ad think of the experiential act as an automatic elaboration taken by Ibjects in their relation with reality. Yet this is debatable. As I have already id, neuroscience has shown the active role played by individuals in the onstruction of mental phenomena, and the influence of the environment in te organism's neurological structure.

/e should not forget the active role individuals play in their experiential zts. Mental phenomena are complex synergetic events whose nature and eaning should be explained via interdisciplinary analyses including tysics, biology (embryology, neural anatomy, neurophysiology, rolutionary biology), psychology (cognitive processes, behaviours), aguistics, and philosophy (of science, of mind etc.). All these disciplines ontribute to the investigation of neural phenomena. When we think of one them as the contingent explanation, we defend an interpretation that is trinsically limited in trying to explain as synergetic phenomenon through a ono dimensional explanatory system.

Physics shows us only 'how' the vision mechanisms are comprised 1 electric impulses (ions). Since the neurosciences attempt to analyse the aain's physical functions, the mechanisms flowing from the neural data ansmission to their elaboration cannot be analysed 'only' by neural rysicists, as their 'area of investigation' is the brain's mechanistic features id not the categorisations of mental events. This means that the :rmeneutic (interpretative) approach to mental events is irrelevant for lysicists, because they investigate the modality/mechanism of neural Ienomena, as opposed to the mind on its own. We could define the rysical approach to neurology as 'analytical,' and the biological, rilosophical, and psychological approaches as 'hermeneutic'. This 
difference between the above mentioned disciplines persuaded physicists to emphasise the exhaustive role of neural physics in a complete description of the brain and its functioning.

Given this, the basic problem is that 'to know the 'wave lengths' of a vision percept does not say anything about the vision of a certain object'. The basic problem is: how can we objectively describe the mind if we do not have access to the qualitative properties of the data that refer to the subject in the first-person, that is, the data from the 'inside'? The crux of this problem is the value attributed to a physical explanation of mind. That is, if we can think of the mind in the third-person, we can objectively describe the mind regardless to its subjective content. In order to solve this problem we need to clarify if a physical explanation of a mental event is a semantic or syntactical explanation. The question reformed as : 'how can the mental first-person content be translated in a third-person description?' When we are physically describing mental phenomena, we are not thinking of their 'qualitative' content. Physical explanations of mental events do not make references to the subject's first-person mental content. They simply attempt to describe mental processes and their functioning. In this sense, we cannot take a semantic translation of a mental event in a physical language, because of the impossibility of knowing the content of a mental phenomenon except from 'the inside' (Nagel 1974). But if we think of this mechanism in terms of a syntactical translation, the physical explanation of a mental event becomes more reasonable: we explain the modalities structures of mental traits without thinking of their content.

When we try to extend a physical (third person) translation of a mental state to include mental content, we substitute a self-reference mechanism with an outside reference. The analysis of mental symbols and the mechanisms of memory show that the mind creates its semantic content by a continuous recategorisation of perceptual experiences. The semantic value of mental symbols, then, cannot be denotative, because mental symbols are qualitatively connoted. The symbolisation mechanisms of mental predicates are thus a biological process of value attribution to experienced data. These values refer, on the one hand, to the subject as a biological organism, and on the other hand to the subject's life as a filter of semantic variations of mental predicates. The semantic value of mental symbols refers to the subject who attributes prima persona their value ${ }^{7}$, but their logical structure can be given a biological description.

The semantics of a mental event refers to a subject and its socioenvironmental conditions (Donald 1993, Lyson 1986). Since the subject is a 
social entity who continuously defines its existence via its lived experience (past experiences, memories, attentions, attribution of value in perceptual acts so on), we cannot defend a view which 'disembodies' the mind (Fodor 1981). This conclusion aims at identifying the explanations of mental phenomena with their contents. And this is not the purpose of neuroscience. The distinction between 'mental' and 'physical' thus is not a juxtaposition between different kinds or aspects of reality, but different linguistic conceptual domains "each of which is associated with its own way of knowing or experiencing" (Goodman 1997: 361, Globus 1973:153-160). The problem is to think of our existence as a dialectic entity: to be an 'objective' physical being and a 'subjective' mental entity.

Let us consider the problem of the dual nature of living beings as an open question. We now need to explain why the phenomenological nature of neural phenomena cannot be explained by neurophysics.

The subjectivity of mental events is a qualitative property of mental phenomena that is linked to the biological-psychological uniqueness of our diachronic existence as subjects. Experiential acts share with biological mechanisms the property of being unique phenomena. Two separate experiential acts, whilst perhaps being similar, will be individuated by the uniqueness of the subject's mind. The subject's mind will never attain exactly the same state at two different times, and this uniqueness is shared by the altered relations between experiential acts. This uniqueness refers to a structural uniqueness (perceptions cause the formation of specific neural circuits and their interconnections) and an originality of meaning (categorisation mechanisms in organisns' perceptions-experiences).

\section{NOTES}

\footnotetext{
'Thus, mono-zygote twins are not identical. Their different approach to experiential inputs causes a difference in their neurotopographies. Suppose both twins are provided with the same sensory inputs (by use of a virtual reality system that supplies the same program to cach of them). Each subject attributes a different degree of attention to some peculiarities of the perceived images, so even in this (extreme) case, the formation of their neural circuits cannot be coincident. The mono-zygote twins' brain structures thus are completely different.

${ }^{2}$ Francis Crick (1989:240-248) and Horace Barlow (1988:571) criticised this liypothesis. The first one said that we have insufficient biological evidence to defend this hypothesis; the second one wrote that accepting this view involves perceiving the evolutionary mechanisms in a Malthusian scusc. Edelman outlines a large number of arguments that push us to accept Hebb's hypothesis. The presence of neural wholes does not imply a Maluhusian vicw because evolutionary dynamics are comected with biological differentiation processes among the member of a species to select the best fitness value. In this sense, Bartow's view that considers
} 
the evolution of the brain as the result of a progressive numeric increase of neural cells, is not justified.

${ }^{3}$ The brain's capacity to manipulate neural maps of sensory information to guide sensorimotor actions is well explained in Baringa M. (1999:189-192).

4 "The interaction between these two systems (RS [reorganising system] and BCSH [behavioural control system hierarchy] assures that the subject shows meaningful behaviours in relation to environment given the activation of physiological systems (...) The set-point of relerence values in the RS are genetically determined (..) whereas those in the BCSII are either genetically determined or individually learned." van der Bos, 1997:151

"Biological forms (explanandum) have internal features connoting their uniqueness. They are self-replicant structures, historical entities, result of natural selection, adaptive (mutable) entities, etc.

"For example, if we think of DNA duplications we see that the deterministic link between the causality of a biological event and its expression is not a potentia-acto phenomenon. If DNA were only a chemical structure regulated by physical lows, how do mutations occurred? Thermodynamics and chemistry describe a direct mechanism: nucleotide $\Rightarrow$ enzyme. So why is this phenomenon mutable? Even if the mechanism that mediates from DNA chemical structure to the production of its correspondent amino-acid is regulated by chemico-physical laws, the possibility of mutation would prevent the simple prediction of the expression of a polygenic character (another example of this structural biological variability are: Jumping gene, or synonymous sequences, crossing over, gametes' selection, affirmation in population genetics of particular genetic recurrences, etc.).

${ }^{7}$ Neurophysiology shows us that all the cognitive systems are connoted in terns of individual variability. This property is also connected to the structural topography of neurons, and the complexity of neural transmissions. These brain-mind's peculiarities show that we cannot describe mental activity by fomal explanatory systems, because we need to conternplate in our view the variability of mind. Even if we describe the mind as a system of representation of reality, we have to clarify how this linguistic system becomes a semantic one. This involves considering how we attribute meaning "internal' predicates, and how this meaning bas been selected via evolution (this last problem obliges us to link brain to biology and to face the problem of social-behavioural link of the subject with reality). 


\title{
I.6. The Mind: A Biological Perspective
}

\author{
"Consciousness is the biological phenomenon that permits \\ us to survey the contents of our minds: our feelings, \\ thoughts and knowledge. (...) [And] there is good reason to \\ believe that consciousness is not only caused by \\ neurobiological states but actually is these neurobiological \\ states." (Damasio 1998: 89-90) \\ "There are at least two sort of views of human agents. On \\ the one hand, the human agent as understanding meanings \\ and acting intentionally, and on the other, humans as \\ biological machines. Modern philosophy of mind argues \\ over whether these two views are compatible with each \\ other, and ... the first is some way reducible to the second." \\ (Perring 1997:175)
}

The Oxford dictionary defines the mind as "the ability to think, feel emotions and be aware of things; the way in which a person thinks." The questions before us are: Is the mind merely an 'ability' with a direct relationship to the brain? What relationship exists between the biological meaning of the Mind and its 'disembodied' properties? I shall analyse some theories that investigate these questions. However, since the literature covering the body/mind problem is vast, 1 will focus my attention upon some of the more significant paradigms in this field.

I will sculpt the discussion around the tightly knitted connection between the mind and the brain, and the necessity of approaching the body-mind problem via biology. This does not mean that I consider neurophysiology as the explanation of mental states. I will defend the thesis that we must think of the mind as a 'composite and synergetic phenomenon' pieced together by interrelated systems. The functions of the mind are multi directed (language, social behaviour, rationality, culture, natural selection, and so on) and are oriented to unify the heterogeneous complexity of the organism, or the Self. This is because "when we think about brain activity it is correct to think about emergent properties at higher levels that depend on lower level phenomena in the system" (Churchland, 1992). Since emergent properties refer to the relationship between higher levels and lower levels of the brain, the central aim of this chapter will be to explore the mutual relations between micro and macro mental phenomena. Why do we have to limit our analysis only to higher levels or lower levels of neural systems?

We should be looking for an explanation that approaches the mental states in their complexity? The analyses found in prior chapters of this dissertation 
are the conceptual platform of my study on brain activity. Thus, my assumptions rely on the neurophysiological features of the brain (chapter 5).

\subsection{Is the Mind a Computer?}

One mechanistic paradigm in neuroscience defines the mind as a computer. This view is based on two central assumptions: (1) the mind is a data container (experiential inputs) and, (2) the reflexive / pre-reflexives body consciousness is determined by neural data memorisation / computation mechanisms. Both processes demand that the brain has a passive role in data memorisation and stability in data management. Is it biologically accurate to say that the brain memorises experiential stimulus in a 'mechanical' way? If we say that mnemonic mechanisms are computational processes we are supposing that our memory absorbs into itself a whole of exact data (inputs). (In a computer, data are 'memorised' via binary systems). According to this, the mind ought to memorise data individually, and its functions ought to rely on the symbolic system that translates reality as it is perceived into brain impulses (Putnam 1988; Dennett \& Hofstadter 1981, Minsky 1986). Edelman (1992; 363) gives a clear analysis of why we may wish to reject this view. Edelman offers two reasons why we cannot accept the mechanistic explanation of the mind. First, the 'brain as a computer' view involves admitting the possibility of directly translating experiential stimulus into a unique and coherent symbolic language (like a binary system). Second, we may find a data claboration mechanism that is similar to those found in a computer. I will investigate both these implications separately, starting with the first problem: What differences exist between a brain and a computer?

When a brain receives sensory data, this information (ionic fluxes) is introduced into a complex system of neural transmissions (neurones, fibres, maps, and feedback mechanisms). In addition, the elaboration of the received information is followed by a mechanism of data symbolisation and phenomena categorisation. The first structure of complexity (memorisation) refers to the second one (elaboration). This involves that the neural events syntactical complexity is reinforced by a semantic complexity. These systems are hierarchically organised into levels of complexity. The brain structure's communication allows the neural data elaboration in organisms that have a secondary consciousness. Since symbolic mental units (for example, mental images) are the result of a structural and semantic neural process, it follows that mental symbols are the result of a symbolisation 
process. This means that even if a symbol is determined on its own (for example, I have my own mental image of my presence), it will still be part of a 'symbolic system'.

Hofstadter (1981), described this symbolisation mechanism as a 'representational system' (a system that can adapt itself to changes in reality). He says that the possibility of linking the variability of a 'representational system' to the variability of the world guarantees the autonomy of mental symbolisation mechanisms. Then the brain can elaborate its representation modules independently of their correspondence with objective reality. This process refers to mechanisms of categorisation and symbolisation. It also refers to an autonomous logic system in which symbols are introduced (Strawson 1997). A computer cannot create a representational system because its relationship with reality is that of passive data memorisation (spatial coordinates, colours, form, etc.). The data symbolisation of something, independently of its 'concrete' existence, is property of the mind. In fact, even in electronic simulations the computer elaborates the received data with regard to the program used. Computers cannot recognise the symbols of this 'simulation' since they receive data passively. Thus, they cannot recognise the differences between simulated data and real data. ${ }^{1}$ According to this, the capacity to have mental images (and also fantasies), defines a vital difference between the computer's management of data and the brain's (think of mental intuitive capacities, Holyoak and Thagard 1995; Rickards 1990; and Stemberg 1990). The creativity of mental symbols is functional to the brain's continuous transformation. This adaptability is oriented to improve rational capacity and adds to the organisms fitness. Computer symbolisation is functional only in regard to procedural systems, and cannot enhance its pre-defined linguistic and functional use ${ }^{2}$.

Another major difference between the brain and a computer is the brain's capacity to substitute its constituents and its general variability. The brain's structure is structurally variable in two senses: (1) neural topography, (2) neural constitution. The first varies depending on external inputs and their elaboration (feedback mechanisms, neural maps, neural connections and so on). The second refers to neuron duplication. Since the brain is composed of cells, their constant substitution alters the brain's topography. In this sense, neurophysiology shows us that we can only think of the brain as a computer in a metaphoric sense ${ }^{3}$. The abundant number of dissimilarities between computers and brains, and the biological features of 
the central nervous system, both deny views that consider the mind as an example of a biological computer.

The mechanistic paradigm often holds that if neural data transmissions and elaboration can be analysed through algorithms, then we could consider the brain as a Turing machine. In order to analyse this problem we have to look at the elaboration of neural data. In a computer there are a series of obligatory steps (commands) for computing the received information (data). The elaboration of one piece of 'information' (constituted by binary elements) is one dimensional. For example, if I program the computer with an algorithm to 'cook the pasta,' I might say to the computer: "Put $x$ amount of water into a pot, add salt, when then water temperature reaches 100 degrees Celsius...", and so forth. However, if some unexpected problem occurs, the algorithm is blocked (for example, we have only 30 grams of salt, and the algorithm requires 40 grams). This is because algorithms are formal systems that do not need direct correspondence with reality. Since, as I stated, brain activity is conditioned by reality, it is a struggle to accept a view that considers human mental processes in terms of algorithms. Neuroscience, however, obstructs us to think of the brain as a Turing machine or mental events as algorithms. According to this, we can conclude that if it is our goal to approach these problems from an abstract or theoretical position, we can use the 'mind as a computer' metaphor. However, if we aim to find an explanation of mental phenomena we cannot forget to deal with the biological features of the brain. When we approach the mind-problem from a biological perspective, the problem of mental phenomena as mechanistic properties collapses leaving us with a second mechanistic view: the mind as a biological problem ${ }^{4}$.

\subsection{Hardware or Software: What's in the Human Head?}

Another reductionistic view states that mental events are logic systems similar to computational systems. They have structural and operative similarities with computer programs. In order to analyse this paradigm, we first need to examine the logic of artificial intelligence $(\mathrm{AI})$ in two different ways: (1) Combinatory logic (Boolean logic), which uses binary systems (input-output) and, (2) Symbolic logic which emphasises the use of symbols in the brain's activities.

Let us begin with the first one. We have seen that neural transmissions rely on ionic fluxes. Neurones receive and transmit these electric messages via their connection among neural circuits. Since neural transmission is an 'all 
or nothing' phenomenon (when neurones are excited by certain electric tensions they transmit received data to axons), we can say that this neurones' property is a mechanism of selective Input-Output (I-O). In a neural transmission the physical dynamic of data-transmission is: $\mathrm{I}-\mathrm{O} \Rightarrow \mathrm{I}-\mathrm{O}$ - ramifications of synapses - $\Rightarrow$ I-OOOO $\Rightarrow$ IIII-OOOO $\Rightarrow$ IIOOOIIIOOOO.... $\Leftrightarrow$ Initial neurones. This mechanism has an incredible formal syntactical similarity to binary systems. Both systems transmit 'information' through coupled links and increase their complexity via updating mechanisms. By elaborating the received information, both use 'blocks of data' as 'units of meaning'. Is this similarity sufficient cause to consider the mind as a biological computer? As computer functions have a dual nature, we must specify if, when we defend the thesis that the mind is a biological computer, we are thinking of hardware or software. Computer software is a system of data elaboration while a hardware is the tool by which the software's data is elaborated on (or memorised). This means that hardware is the 'structure' of memorised data and the vector of data transmission for the software. (Take the case of a cash register. The hardware is the 'object' cash register, the software is the numerical data we introduce in it.) According to this, we can think of the mind as a computer analogy in two ways: mind $=$ software, or mind $=$ hardware.

The first view considers mental events (memories, passions, believes, and so on) as a set of data contained in the brain (Leiber 1980). This container describes the peculiarities of a subject, regulates the (neural) data elaboration mechanisms, and requires a constant coherence in our mental activities 5 . The second view claims that we can then think of the mind as hardware (Dennett \& Hofstadter 1981, Hofstadter 1979). The structural organisation of my neural data becomes a key to explaining the mechanisms revolving around mental states. Even if external inputs (bits) or the mental software (my approach to experiential inputs, or my memory) both change, the data transmission and elaboration structure does not vary. Given this, the brain's activities remain structurally the same, for if the mind is hardware, the memory is merely an up-dating phenomenon. The neural data's semantic elaboration is explained in the cumulative mechanism transforming a set of simple neural stimulations into global information whose meaning relies upon the initial input of information. Thus, I can explain complex mental phenomena by analysing micro-information (protoinformations).

I shall discuss both these options. If we think of our mind as software, we have to think of a brain as a biological structure not influenced 
by neural data. All brains ought to be structurally similar (like the computer in a computer farm) and an organism's individuality ought to become a requirement a posteriori. Thus, we all had an identical brain at the moment of our birth, and this structural identity was transformed into uniqueness because we have this indefinite software regulating the mental events.

The first objection to this view is biological. In the foregoing chapter I have emphasised that, even if the topography of our brain refers to our perceptual inputs (because they influence the neural circuits' structure), nevertheless the brain's topography is absolutely unique. We have numerous biological reasons for claiming the brain's structural uniqueness. And this individual property is reinforced during the organism's life stages. The mind-assoftware view thus has to explain the brain's uniqueness (if we think also of the experiential inputs' categorisation mechanism, we see that the mental syntactical processes have a grammatical and structural uniqueness also).

The second objection to this view refers to mental diseases. If we think of our mind as software, numerous brain anomalies (pathologies) are paradoxical (Williams 1979, Lurjia 1973; McCarthy 1990). For instance, if our mind is a computational program, when I have an irreversible (or temporary) amnesia, is my mind destroyed (Olson 1997)? If this program contained my individuality, when it is damaged, I ought to conclude this software damage to extend to all my mental activities (my mental identity is destroyed). According to this, it would be really difficult to accept my mental continuity, since I have lost my memorised data by substituting my initial software with another one that contains my mental traits. My mind would be interrupted and substituted by another one completely independent of my initial identity (Nooan 1989, Mills 1993). Take, for example, a patient whose hippocampus is removed to treat lethal epilepsy. Since he cannot recall his/her long-term memory (Edelmann 1992), we could interpret his dysfunction as a succession of continuous 'black holes' in his relationship with the reality. Thus, we ought to think of a psychological continuity as the cohabitation of independent and different Selves. The psychological continuity would be destroyed ${ }^{6}$ (Damasio 1998). We can radicalise this view. We can talk about our mind as a set of neurones having in themselves an indicative value in our mind (Minsky 1986). Since software is explained via its sub-requirements, the mind might also be explained as a whole of neural identity units. Do we have to conclude that the mind is a set of independent sub-units? How can this puzzle continue over time if its subconstituents change? 
My final criticism of this view rests on the nature of memory. If we think of mind as software, it follows that my memories are the result of neural data organised and mediated by my 'mental program'. Computers have this kind of memory: the memory as a 'container' of information neutrally memorised (they do not have to have undergone qualitative variations). In computers, an image is memorised in binary elements e.g. 10-111-000-10101000001-0001010111100. The global dimension 'image' contains this simple information. We can decompose the image in its subunits, and re-build it by retaining the pixel order. The brain's memory is different ${ }^{7}$. Even if an image is composed by simple neural data, the brain does not compose an image by accumulating the above mentioned data, but by symbolising and interpreting the data in the unitary dimension of an 'image'. We do not know where and how this translation is taken, and we cannot decompose the image in its simple sub-units (proto-informations). In the same way, we cannot remember the majority of the acts we have done yesterday (Parfit 1984), because our memory uses our past experiences by following a categorisation mechanism (Edelman 1992). It attributes a value to our past. The memory interacts qualitatively with our past, and this mechanism cannot be compared with software processes. This phenomenon is evident when we think of feelings. How can I explain the intentional choices I have made yesterday through a mechanistic schema"? And how can 1 explain the existence of mental images in our mind if the capacity to visualise something strictly refers to a visual mechanism? How do we explain imagination? In considering these criticisns, I believe we have insufficient reasons to support the mind-as-software view.

Let us now consider the possibility of the mind-as-hardware. This view has been called 'functionalism' (Putnam 1975; Jonson-Laird 1988; Pellionisz \& Roesenfeld 1988, Fodor 1981, 1996). The central assumption of this theory is that algorithms can explain neural events. These algorithms should be computable by a 'Turing machine'. I have criticised this view in the forgoing pages. I shall approach a similar problem: can programming languages translate mental events? Artificial intelligence uses different programming languages. The design and animation computer programs ('AutoCAD', for instance) use linguistic symbols in their strings. Composite information is symbolised in particular 'words'. In 'AutoLIST' (the programming language of AutoCAD), for example, symbols contain specific information. ' $A$ ', in my string, could be 'to calculate the geometric 'place' of points in a Cartesian system'. 'A' aims at drawing a circle. The A-predicate's semantic value thus is determined and defined on its own. 'A' 
symbolises a composite data elaboration, and it remains the same in all its uses. The A-predicate does not change, it contains data having a determined succession and structure. This involves that the use of a symbol in a computer is only a tool to compute certain composite information. Computational symbols are not variable 'units of meaning'; their existence aims as defining a syntactical system in the data elaboration. Edelman (1992) defends a really fascinating theory on the mind's functioning. $\mathrm{He}$ stated that the brain is an integrated locus of units and diversities forming a global dimension that contains the Self's peculiarities" (memory, self awareness, rational activity, and so on). He approached the mind's peculiarities via neurophysiology. We can synthesise his theory as follows: the sensory inputs cause the change in topography of cortical maps, and this variable structure evaluates the received information by continuous interconnections within the brain's sub-units. Experiential body enriches the brain structure's degree of complexity, and in doing this, it allows the formation of composite (and complex) mental processes. This involves that the brain has a hierarchical organisation in series of feedback loops (Power 1973, Cools 1985, Hebb 1980) through which functional neural paths are retained according to the principle of selection (Edelman 1992, van der Bos 1997). This mechanism produces a mental semantic capability - a conceptual categorisation - in the brain's neurocortex. The mental semantics are based upon neural data and its subsequent elaboration received via sensory inputs. 

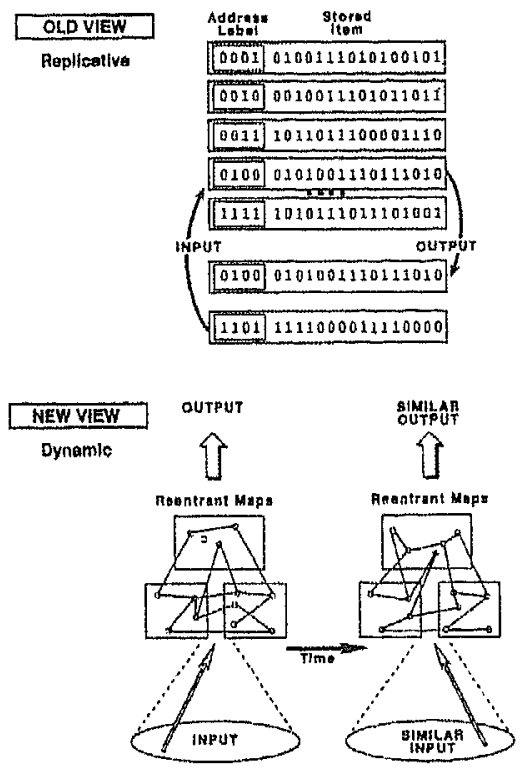

This ligure shows the old view of memory as a replicative system, and the new view that stresses the dynamic nature of memory, (Edelman 1992:103)

The debate over the symbolisation mechanisms in the brain is not concluded (Pinker 1999). We know that the individual's consciousness at certain times depends on which cortical area is active at these times (Dennett 1991, Pinker 1999). But what we do not know for sure is how neural data are packaged in meaningful information, and if a central mechanism exists in sensory data management (Dennett 1991, Kinsbourne 1995, der Bos 1997). When a brain symbolises something, it uses a plastic dynamic ${ }^{10}$. Even if the system of sensory inputs is a binary system, a really complex set of neural connections computes neural data. (In order to have an idea on the complexity of this process, note that the connection between the right and left hemispheres of a brain is composed of around 200 million fibres). Since this data elaboration is performed by interactive neural maps, and the memory categorisation process is a continuous re-categorisation of accumulated information (memories), it follows that different (qualitative, structural) neural associations change the semantics of mental symbols. The information process in the mind is not a one dimensional mechanism 
passing from an initial simplicity to a cumulative complexity, but a process of plastic fitness to external reality.

The probabilistic meaning of this data categorisation makes the linguistic use of symbols in the brain unique in each individual. This shows a basic difference between the computer's memory (based on consequential processes) and biological memory (based on semantic processes). This difference exits at two levels: the symbolic level (software) and the structural level (hardware). As I stated, neurophysiological evidences suggest that neural inputs 'cause' the structural interconnections among neurones by which neural data are transmitted and elaborated. The plasticity of this semantic mechanism relies upon continuous changes in the syntactical structure of the data elaboration processes. This involves that the brain structurally varies in its topography. This structural organisation is the transmission system via which neural data are introduced and successively elaborated. This system is structurally conditioned by neural inputs and is the syntactic structure of their semantic elaboration. Thus, the brain's topographical structures constitute the syntax of neural data elaboration, but the semantics of this process is not explained through the use of mental symbols alone, but relies upon the same topography of neural data transmission. Computers do not have this capacity since electronic circuits have a structural function regarding the data transmission and computation that cannot change in the face of variability in the inputs. Electronic microcircuits transmit information, but the structural conditions of this elaboration cannot vary. The computer software allows us to compute variable data, but the possibility to change the 'use' of a computer is based on the impossibility of changing its electronic configuration. This means that hardware, whilst being the 'vector' of data transmission computation, cannot adapt its structure relative to the received data.

A computer is an 'object' undergoing reality. Computational systems cannot modify themselves relative to their relationship with the world (if we connect a camera to a computer we cannot change its hardware). The brain possesses this plastic capacity. Neurones are changed, substituted, and organised in 'variable' ways (neural circuits) depending on external inputs. The basic difference between a brain and a computer is the inability of inorganic matter to adapt itself to external conditions and to reproduce its constituents. To the contrary, the brain reacts to environmental and social inputs so as to increase its fitness. The brain also has the capacity of being a self replicating structure. And this is a property absent in inorganic systems (also crystals ${ }^{11}$ ). The brain adapts itself to reality, where computers merely 
undergo it. In realising that the mind is not a computational system, the claim that the mind is an updating-data system becomes nonsensical. The mind is not a 'container' of experiences and experiences are not directly translated in memories. The experiences interact with the neural systems, and this mechanism transforms either the subject, as an 'experiential body', or its memory. According to this, the cerebral topography is composed by numerous structures that change continuously in response to individual experiences. The relationship linking ourselves to our memories is plastic (Baillic 1993, Parfit 1984).

\subsection{The Role of Memory in the Mind}

So far I have stressed the difference between computer memory and biological memory by emphasising the computational systems" memory. Let us look more deeply into the features of biological memory. Memory is the 'capacity to repeat an act'. It is a process of continuous redetermination of mind. In human beings this process works at two levels: the physiological and the psychology level. The physiological level is necessarily plastic because the neural interconnections change through time. The sensory inputs arrive at the cortex. There are tens of billions of neurones, each connected to thousands of other neurones via more than sixty thousand miles of wiring (Lewin 1992:163). They are organised into maps and connected via three structures: (1) The cerebellum, that receives inputs from the cortex and spinal cord and has the function of synchronising (the successions of) movements. (2) The basal ganglia, that regulates movement. (3) The hippocampus, that plays a crucial role in spatial memories and contributes to memory consolidation (the conversion of short term memory into long term memory.)

Different kinds of memory exist within biology. There is genetic memory (data encoded in chemical form), cellular memory (e.g. the immune system), neural memory (for example, the constitution of circuits), mental memory (that is, memory in the standard sense) and primary and secondary consciousness. The memories that link an organism biologically, psychologically or linguistically (semantic bootstrapping) ensures that organism's continuity. Memory is 'a whole of plastic variations of biological systems' structure / organisation. It is a process oriented towards two biological ends: (1) unifying the biological unity-diversity, and (2) regulating its continuity. 
At the same time, memory is only one element co-participating in the heterogeneity of organic phenomena. The same way in which I deny that DNA is the basic element of biological individuality, I deny that 'mental' memory explains all the peculiarities of mind's, since it is the result of numerous biological processes. According to this, it is very difficult to reach one unique property that explains our biological and psychological identity (Wiggins 1980, 1987, Di Francesco 1998). This biological analysis shows us that our existence refers to synergetic processes that co-participate in ensuring our continuity. Thus, the personal identity problem has a bipolar connotation. The identity is a synergy of biological and psychological 12 processes having an analogous purpose of unifying a heterogeneous composite dimension in a homogeneous entity whose qualitative structural features vary without changing the individual's continuity. From this perspective, biological reductionistic theories (organisms as machines and mind as computer) become limited explanatory tools to analyse the organisms' biological individuality, since living beings are entities that adapt themselves to environment and memorise these environmental influences.

This means that our actions influence our biological and psychological constitution. This process makes an individual a plastic dimension that continuously changes by adapting to reality.

Memory does not only refer to neural connections, but is mediated by other systems controlling its expression and sedimentation in a subject. Numerous biological reasons induce us to accept the interrelation between the neurophysiology and psychology (Dennett 1991). As mental states have a continuous qualitative and structural variability, memory is qualitatively and structurally variable ${ }^{13}$. This plastic relationship changes our constitution.

\subsection{Self-Awareness and the Unity of Consciousness: the Biological Perspective}

Here I shall analyse a central problem of philosophy of science the role of self-awareness in the existence of individuals. I will claim that both the environment and experiential phenomena influence the subject's Self synthesis. This analysis aims at discussing personal identity by basing personal identity problems upon rational capacities. I will analyse the biological mechanisms of Self-synthesis in order to criticise some theories from philosophy of mind. 
In 1890, William James wrote that consciousness has specific features: it is personal, changeable, continuous, it analyses external objects, has intentionality, is linked to wishes. Consciousness has two levels of complexity: (1) Primary consciousness (the capacity to have mental images of reality based on perceptions at a certain time), and (2) Secondary consciousness (the capacity to have mental images of reality and an individual self awareness).

Primary consciousness is a reply to external sensory stimulus, Organisms having a primary consciousness base their present actions on their past experiences. Their behaviours are linked to their past. Nevertheless, they cannot reinforcc this mechanism with self awareness capacities. They have the consciousness of their presence-in-the-world, but they do not have the capacity to imagine themselves as an individual. They cannot create mental symbols of their individuality (Edelman 1989; Griffin 1984, Premak 1983). According to this, in primary consciousness individuals, the memory constitutes the vector joining the organism's past experiences in a unitary self deprived of reflexive self-referential properties. This mechanism raises an individual's fitness. The subject uses its primary consciousness to adapt itself to reality through continuous recoveries of its past experiences. This process is based on specific neuroanatomical structures, since the memory constitutes a structural link within neural maps, and increases the capacity to unify the internal neural circuits by a net of neural interconnections. A clear example of this mechanism is an animal's capacity to repeat complex sensorimotor actions. Organisms possessing primary consciousness cannot self-reflexively refer to themselves, because their self awareness incapacity obstructs self-synthesis process. These individuals thus cannot create a (memorised) mental symbol of their existence ${ }^{14}$. The capacity to translate an organism's identity into a mental symbol constitutes the conditional property of passing from primary to secondary consciousness ${ }^{15}$. When we say that reflexive self awareness presupposes an individual's capacity to create the mental symbol of its own existence, we are not denying the possibility of a primitive - pre-reflexive - self awareness. The rationality active in the capacity to repeat complex sensorimotor behaviours involves a sensorimotor form of awareness. However, the growth of the synergetic complexity of mental phenomena in different animal species individualises their self-awareness (at an individual specific and species-specific level), and differentiates the complexity of their mental existence. Those organisms that do not have conceptual awareness do have a form of sensorimotor awareness (Meijsing 1997:57). The awareness problem among different 
animal species, becomes a question of differences in levels of complexity (Den Bos et all. 1997, Gallup 1982, 1997, Heyes 1993). Animals are conscious but their awareness is qualitatively and structurally different from that of human beings. Comparative analyses of inter-species awareness, and causal factors interacting in an individual's self awareness synthesis, can be applied in natural taxonomies.

Let us now analyse the second level of consciousness. We have seen that organisms with secondary consciousness are aware of their individual existence over time. They can symbolise themselves as individuals and are aware of being the subject that 'owns' their experiential acts. Individuals with primary consciousness can attain secondary consciousness only if they develop the capacity to translate their experiences in a symbolic sense. They need to be aware they are an experiential body - a subject - and a memorised Self. According to this, the link between primary and secondary consciousness is the capacity to create a symbolic tool in which primary consciousness experiences can be introduced, used, and interpreted by a self aware subject. This linguistic (or semantic) process is reinforced by the social interaction with other individuals, since social relations allow an exclusion mechanism. A subject learns to be an individual by excluding its identity to the identity of its interlocutor. How did human beings create the symbols of their existence?

The biological development of the human vocal apparatus is the result of epigenetic mechanisms. The evolution of neurophysiological structures ${ }^{16}$ and the phenotypic development of the neural pharyngeal apparatus allowed us the physiological capacity to talk. 


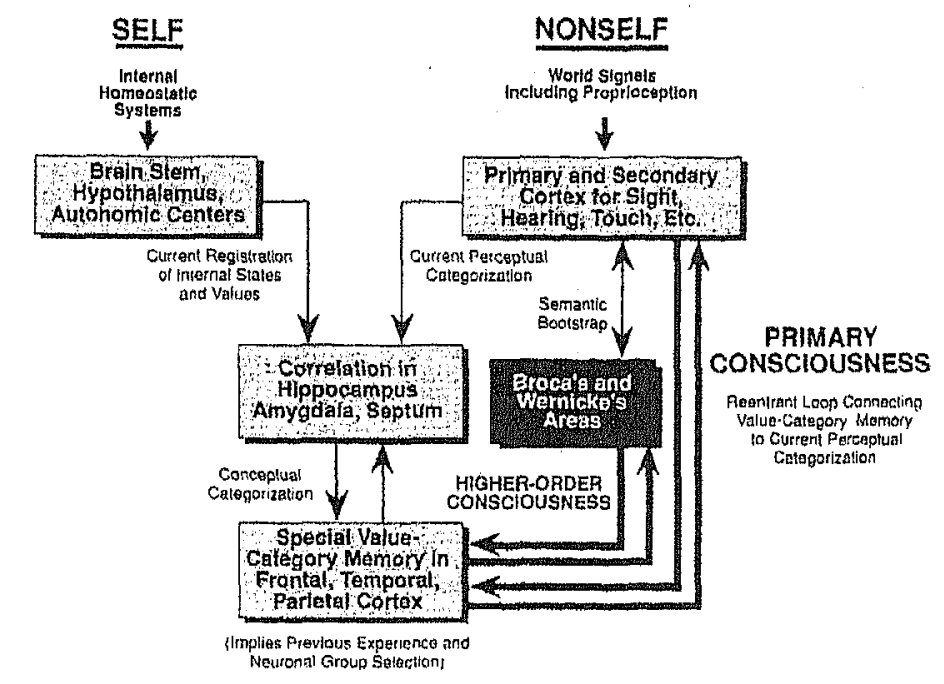

(Edelmann 1992:132)

At the same time, the link between phonological symbols and concepts determined the semantic (after the syntactic) processes of our linguistic systems. When this process was concluded, the brain connected the word's semantic meaning to their denotative meaning, and this process aimed at defining the syntactic structure in which we introduce our language. The memory absorbed this mechanism and learned how to use this process for communicating. At the same time, the recognition of linguistic meaning relied upon the capacity of memory to use mental (symbolic) translations of morphemes. Pinker and Bresnam labelled this process 'semantic bootstrapping' (Bresnam 1982, Pinker 1984).

Here it is not my intention to analyse the linguistic problems ${ }^{17}$ of semantic bootstrapping, I only aim at emphasising that the linguistic system is the first conceptual representation of an organism's identity. Let us consider the linguistic elements allowing human self awareness during infancy ${ }^{18}$.

Brunner (1978, 1981:1-13) analysed the linguistic relationship between adult and infant. He observed four consecutive steps in the subjective self determination:

1) The infant is recognised by the adult (You are Maurizio). 
2) The infant recognises the adult (you are my daddy).

3) The infant recognises itself as the 'recognised person' (I am that person that my daddy called 'Maurizio').

4) The infant recognises itself on its own (I am Maurizio).

This analysis highlights two important consequences: (1) The human reflexive self-reference is a process of exclusion to an interlocutor. (2) This process implies the presence of other individuals (it is a social process). The reflexive self-reference refers to the presence of another subject. The Selfsynthesis from its inside requires the perception of an external reality. This mechanism involves a dynamic 'inclusion exclusion' mechanism: (1) I know myself to be Maurizio since I am something different from you. (2) I know myself to be a human being (and not a cat) because I recognise myself in you. (The second step of the 'inclusion exclusion' mechanism refers to inferential deduction) Given this, individuals with secondary consciousness require social relationships to begin the processes of self awareness and linguistic capability (Boyd \& Richerdon 1985). This mechanism begins through the adult-infant relationship (Markova 1990, Rochat 1995). This involves my learning of my own individuality via my exclusion of others, and I successively memorise my existence in Self-synthesis processes ${ }^{19}$ (Wilkers 1988).

In a biological perspective, the experiential inputs that I received in seeing my father interconnected my neural maps. I symbolically learn that the other person (my father) talking to me is 'something different' than myself. When I pass from the first two steps of Brunner's schema (1-2) to the third one (self-recognition), I enrich my neural connections by feedback signals allowing the semantic mechanisms to allow for my linguistic selfreference. I learn the use of the morpheme ' $Y$ '. At a later time, the fourth step of Brunner's schema (my self awareness) will be done when the mental symbol of myself is topographically constituted. When this process is concluded and memorised, I can re-use it over time. The memorisation of the inclusion-exclusion sociolinguistic mechanisms allows me to be alware of my identity and to symbolise it. My Self-synthesis refers to my being-inthe-word.

Secondary consciousness synthesis thus can be schematised as follows: (1) Perceptual data cause the formation of neural maps. (2) These maps communicate with the other (memorised) maps - feedback mechanisms. (3) The result of this process is a mental symbol that is memorised. (4) The organism bases its Self-synthesis processed upon the mental symbol of its 
own existence. This is a synergetic linguistic, perceptual, cognitive, social process $^{20}$. We thus learn our identity via the inclusion-exclusion process, and successively enrich this mechanism through sensory categorisation mechanisms. The continuous change of weight (meaning) we attribute to our experiences influences the Self-synthesis process. Self-synthesis is a dynamic process changing through time.

\subsection{Self-Synthesis and the Unity of Consciousness: the Philosophical Perspective}

If we consider self awareness as a reflexive self referential peering into a subject, it follows that we should underline the individuality of a subject (Lakoff 1986, Johnson 1987, Searle 1992). In this view, the outside reference is merely an indicative identity description. The lack of possibility to actually know the subjective psychology obliges us to think of individuality as an unknown element that strictly belongs to a subject on its own (Nagel 1986, 1973, Wiggins 1987). We can thus only look for the maintenance of some physical requirements of an organism as minimal conditions to maintain a self-reflexive capacity (for example, the way in which the central nervous system functions). However, these parameters only control the self-reflexive mechanisms in their physiology and do not describe the psychological entity from its inside (Fodor 1996). Do we then conclude that identity is an unknowable concept? If the identity subjectivity can be analysed only from-the-inside of an individual, why are we even talking about identity (Parfit 1984)? What about individuals having some mental dysfunction affecting their psychological continuity"?

The answer to the first question is that the intemal reference is a property belonging to every organism, so we cannot know the identity of another organism from its constitution, but we can know the processes that construct its identity (Wilkers 1988, Nagel 1986). We can only clarify the role and the function of self-reflexive mechanisms within the synergetic and heterogeneous biological properties that denotes an organism as an individual living being. The problem in attempting to minutely describe identity features becomes insignificant. We cannot exhaustively describe a biological individual on the merit of its biological constituents (let us think of the analysis of organism's biological features). By the same token, we cannot describe the reference from-the-inside of individuals (Nozick 1981). Our attempt aims at individuating the mechanisms and the properties by which an organism is an individual entity in itself, and not at describing its 
own requirements. These elements cannot be exhaustively described because of their continuous structural and qualitative changes.

Since we know psychological identity is a requirement of an individual, how do we conceive of the individuality of anencephalic children, embryos, Alzheimer patients, people with a dissociated personality, ammestics, comatoses, and so on? There are two possible solutions: (1) we automatically deny their individualities, because they are not self aware and rational subjects or, (2) we look for a further element that constitutes per se the meaning of their existence. The first conclusion is strictly linked to Kantian philosophy. The absence of a cerebrum and its mental activities is projected onto the global entity, an anencephalic infant (Nooman 1997). The second conclusion isolates a disembodied element that contains the basic value of life on its own (the argument of 'Sacred Life' or Cartesian dualism). Both of these conclusions are problematic. On the one hand, the absence of rational activities does not deny the existence of a rational individual $^{21}$ but its conformity with our ideas on what distinguishes a human being from another living creature (Wiggins 1987, Donald 1991). On the other hand, we can accept non-reductionistic views only in their tautological basis (Hare 1952). Neither of these avenues talk about the subject's individuality, but they do consider the value we attribute to an organism to consider it as an individual. We cannot deny the individuality of an anencephalic infant, or of an Alzheimer patient, but we can evaluate their existence using an external point of reference. In attributing a conditional value to our outside reference, these problem cases individuate the existence of individuals who do not have a sufficient conformity with "what is really important to be an human being" for us (e.g. volitive, rational, and reflexive capabilities). Yet, this does not mean that they are without identities.

If we accept the value of internal references, we should at least leave a margin of autonomy to these identities. We should allocate them a value based on their own constituents, even if we consider this value to be a minimal one. I believe the conclusion about their identity values will be a radicalisation of the mechanism of outside reference. We ought only to remind ourselves that the outside reference (external) is a relative system (Nozick 1981, Nagel 1986) referring to cultural as well societal factors (Lycan 1996). Time and culture could change what is important to be an individual (think about the debate over animal rights). According to this, I believe that identity theories (including mine) are merely philosophical explanations. 
If we accept that a particular feeling, belief, or mental condition influences our perception of reality, we can accept that I am the vector of formation of my psychological identity, and that my mechanisms of memorisation influence my approach to reality in different ways at different times ${ }^{22}$. I have an active role in my relationship with my past or with the present of my perceptions (which will be memorised). By the same token, my psychological continuity is not linked to a certain quantity of memories, it refers to the relationship between me and my past selves as a variable process $^{23}$ (Mills 1993, Noonan 1989, Parfit 1984, Nozick 1981).

\subsection{Self Reflexivity and Self Awareness}

When I see a table I can talk about its identity as that table. The table cannot draw this same conclusion. The possibility of reference fromthe-inside is conditioned to the presence of a Self. Even if my identity "for the others" is submitted to their outside references, my mental activity allows me to think of my identity from-the-inside. In this sense we can say that reflexive self-reference refers to its agent, and on the reference processes (Nozick 1981:114). In other words, our reflexive self-reference implies that we have a reflexive self knowledge and that we are agents of self reflexivity. When we question our individuality, we refer to ourselves. We solve the problem of self-reference via the question that we previously asked. As this question is internal to the agent of reference, it follows that the possibility to interrogate ourselves on our existence involves the existence of a subject, and the existence of a reflexive self knowledge. Thus, questions about our identity imply the existence of a Self that is self interrogating. Since the Self performs and enacts this process, we can conclude that the Self exists and that the reflexive self-reference is an inferential mental property that is based on the existence of our Self ${ }^{24}$ (Wiggins 1987, Nozick 1981). To be a Self, is to have the capacity for reflexive self-reference. We cannot explain our existence in any other way. How could we wonder about our existence without using our Self? How could we approach these questions by using an entity that is external to ourselves? If we radicalise the role of the reflexive self-reference as merely an internal point of reference (from-the-inside) we arrive at contradictory conclusions and deny the continuity of people who loose consciousness of their psychological continuity (Wiggins 1980:176-178). Nevertheless, the 
self-reflexive property of a subject is a basic mechanism in identity mechanisms.

If we think of identity in terms of a mental entity we risk losing the dual nature of identity: to be both a biological organism - a concrete entity - and a mental entity. Kant attributed the strongest value in the existence of a subject to this last concept, but the acceptance of this radicalism remains problematic (Salvi, 1996:122-3, Justice 1994:1227-9). When we approach our identity pragmatically, we need to clarify the role of our reflexive selfreference within the global identity process by which we are the subject of our existences and the physical (biological) entity that lives in the world. In parallel, as our psychological identity is not simply a mechanism of selfreference, we ought to think of it as heterogeneous dimension containing rational or emotional activity and the reflexive self-reference or our beliefs (memories). The cohesive heterogeneity of a Self suggests that we think of the reflexive self-reference as a platform upon which psychological identity is based, but not completely explained. In this sense, our self awareness is important because it is an element that unifies our psychological identity. This active role interacts with the unification process of our identity. Congruently, it increases the degree of unification of the organic unity.

When a subject is deprived of its self awareness its existence is deprived of a basis of consciousness. The psychological identity of this unconscious organism does not have a unitary value because the heterogeneity of mental states does not posses an internal point of reference (Bisiac 1988, Bisiac \& Geminiani 1991). Why should the absence of rationality destroy the subject's unity of consciousness? Why does rational activity form the basis of the existence of a subject as a subject?

The problem is that 'because of' our status as 'rational agents', we suppose that our self-reflexive mechanism exemplifies the 'only' way to be a Self. Since our reference from the-inside is based on rationality (or other mechanisms such as linguistic capabilities) we consider rationality as a prerequisite to be considered as individuals. In doing this, we are radicalising the role and the value of a human process as 'the' metric to be a Self and to be an identity. (On animal self awareness you can read: Griffin 1976, Premak 1983). We individuate to an outside-reference the conditional property of being an individual. However, since the self-reflexive capability is only a component of the complex Self-synthesis process, I believe that we cannot attribute a conditional value to it. This does not mean that a person who has brain damages maintains its psychological continuity, but rather that the biological nature of an organism as an organic unity, like the 
psychological identity is a unity-in-diversity. The absence of a self-reflexive property does not deny the existence of a psychological identity, it simply influences its degree of unified complexity. We can be aware of our existence in different ways and at different levels.

If I have amnesia I lose psychological continuity and my self awareness will weaken in its historic nature. I will be conscious of my existence now and not, say 20 years ago. However, I will maintain a degree of self awareness that describes my psychological identity and its continuity. I simply have a ramification in my continuity. Since the psychological identity of an individual is not purely the result of a 'mnemonic database' (Parfit 1984), the mono-directionality of the constitution-continuity of a Self is denied.

If we agree that there is a possibility that a particular experience can influence our psychology in its double constitution (the Self, and the memory, Edelman 1992), we admit the existence of a mutual relationship between my memories and me. If I have a shock (for example, causing the death of a partner by transmitting the HIV virus) I interact with reality in a particularly different way. For example, I feel the importance of my Life's moments, and revel in the beauty of Life (Nature, love, child, art, and sex). I influence my perception of reality as I am an agent of perception (Brown 1977, Lurija 1973, Hidelgard 1977). Of course, I cannot influence my sensory experiences (if 1 touch a glass, I cannot interrupt this perceptual experience), but I can influence my qualitative approach to these phenomena. When I memorise these experiences I privilege those that were important for me at that moment.

As we influence our memory, we change our psychology, and some 'shocking' gaps of our life can concretely change our psychology and our relationship with reality. The problem is located in the $r$-relation that joins the facets of my Selves. The impossibility of thinking of r-relation as a one dimensional process involves the possibility of a ramification in my continuity of existence. In the same way, if I suffer from schizophrenia, I will be conscious of myself, but this consciousness will weaken because the r-relation linking my different multiple selves (through time) will be not coherent (Braude et all 1995, Lizza 1993). This gap could lead to confusion. For example, one time I may think of my psychological identity as Maurizio, and at another time as Julius Caesar. However, I will be an individual independent from the variability in r-relation. What really changes in this case is the degree of my unification. In cases of amnesia and mental disease, my self has a minor degree of unification because its continuity has "leaps, and wholes", and my self-reflexive property is highly 
mutable. If we emphasise that it is impossible to know the subject's content of consciousness, and we agree upon the importance of the reference fromthe-inside, then we should substitute radicalism on rational activity, with another metric which stresses the variable and composite nature of our psychological identity ${ }^{25}$ (Lyson 1986). Thus psychological identity is not the exhaustive property of identity, but it is a basic mechanism of an organic unity's unification. As the organism unifies itself by a causal internal rrelation the psychological identity unifies an organic unity as a cohesive and variable entity. The individual self-reflexive property unifies the mental entity of a subject and constitutes a mechanism of its unification.

\subsection{Philosophy and Neurology}

When we explore the neurophysiological feature of Self-synthesis we see that several philosophical theories on Mind collapse. Think of Locke's view (the memory as a container of experiences), Hume's view (the Self as a composite dimension deprived of an internal unity ${ }^{26}$ ), and Kantian radicalism. Locke wrote ${ }^{27}$ that we begin with the human mind as a tabula rasa. According to this, our knowledge capacity is based on ideas resulting from perceptual inputs. The experiences we have during our infancy are the milestone of our rational capacity. If we approach this conclusion in a biological perspective we see that Locke's position is only partially correct. If our mind were an 'empty page' we would be born without pre-defined behaviours. However, Lorenz's issues on animal imprinting (Lorenz 1973) show that the Lockean view is incorrect, since we are born with preconstituted neural connections and 'instinctual' behaviours. Since the mind is the result of the neural groups' natural selection oriented to improve the individual specific adaptive capacities, it follows that we inherit a speciesspecific brain structure and we successively enrich it by our experiential inputs. This process defines our neurophysiological identity.

The topographic structure of our mind is the result of selective pressures that selected the neural systems we inherited. This means that cerebral structures refer to the genetic code contained in the encoded information within the topography of our brain. Then we have an individual specific diversity, reinforced by our life experiences, and a similarity among the members of a species. This bipolar biological connotation also exists in our neurophysiology. We cannot think of our mind as a blank page (the Lockean view) because it receives a whole of information preceding the 
subjective 'experiential acts'. On the other hand, if we think of our mind in a biological perspective, we see that we inherit a specific neural topography that structurally supports the neural phenomena and successively changes relative to our experiences. In this sense, neurophysiology helps us to investigate the peculiarities of Selves.

In parallel, the formation mechanisms of secondary consciousness (Edelman, 1992) and the Adult-Infant linguistic mechanisms (Brunner 1981), can be used to analyse Fichte's view: 'the Self is the agent and the result of its reflexive self-reference'. In fact even if Ficthe's view is partially correct (since the Self synthesises itself from its inside), the Self-synthesis mechanism refers to the subject on its own 'and' its relationship with reality (linguistic consciousness, neurophysiological circuits and values resulting from perceptions). The inputs to secondary consciousness come from the external (experienced) reality, but their categorisation and connection are done within the subject. The value-distinction between external-internal (the Self, the experiential body) becomes nonsensical, since both contribute to the unitary process: the Self-synthesis and its continuity.

It is not possible, then, to describe the identity of individuals as a specific system. In fact the individuals' biological and psychological features co-participate in their existence-continuity. And the variability of environmental conditions so strongly influence their existence that we can say they are qualitatively, structurally, and topographically mutable. We thus have to think of identity as a process regulated by the internal $r$ relations clustering the individual biological-psychological diversity in a unitary entity that continues through time. This process defines our individuality and is the result of a historical mechanism. In the brain, we inherit a biological and neurophysiological structure that is continuously enriched through experiences and their categorisation. Thus, we organise our biological and mental identity (our 'inner being') depending on 'external' reality. This means that it is not possible to define an organism as an individual according to rigid classification criteria. It is important to be aware that an organism is a composite and variable dimension formed by a biological and psychological diversity unified in a unitary dimension: individuals are organic units. Since personal identity features (and their value toward the individual life) vary, and this is a natural process, we only can to approach the plasticity of individuals' existence in itself. According this, we have to approach the identity problems by thinking of the existence of individuals as a process. In this process the internal $r$-relation among individuals' constituents becomes central. Its role is to unify the organic 
unity 'individual' in its biological and psychological dimensions. Both the biological and mental systems are connected and both share an analogous goal: to unify the organic unity 'organism'.

\section{NOTES}

'Philosophers have explored the differences between features and capacities of humans and computers. Nagel, for example, (1973), considered the computer's incapacity to lie as such a difference. Morton underlined human subjectivity (1980). Other differences are creativity, feelings, and volitive capacity, amongst others.

${ }^{2}$ Patricia Churchland tried to link neurobiology to philosophy of mind (1986). She has analysed the differences between minds and computers (Churchland 1992). Her central thesis is that mind and computers are different since "The nervous system is a parallel-processing device, and this conveys several interesting properties. For a start, signals are processed in many different networks simultaneously. Next, neurones are thernselves very complex little analogue conputers. Last, the interactions batween neurones are non-linear and modifiable. Real neural networks are non-linear dynamic systems, and hence new properties can emerge at the network level" (Lewin 1992:165)

${ }^{3}$ Then, we are aware that the brain is not a computer 'and' we merely underline the existing similarities between neural systems and computational systems. For example, we can emphasise their data processing capacities, but this comparison is limited to functional similarity and does not defend the initial analogy (the brain as a computer).

4 " $A$ reductionist account of mind has become more popular with recent advances in neuroscience. That is to say that the way the rnind works can be described in terms of the way the brain works. The brain is constructed out of a huge number of neurones. Each neurone is connected to a large number of other neurones, forming a complex structure. Individual neurones are cither in a state of firing, or not firing. If the mind can be described in terms of these neurones, then it seems likely that the actual mechanism by which a signal travels in the brain is unimportant. The structure is what is important, not the actual physical constitution. ... parts of the brain could be replaced in cases of brain damage with a small microtransmitter which has the same function as the neurone it replaces. When a signal enters the microtransinitter, it acts just as a neurone would and passes the signal along to other neurones in the same way that an actual neurone would. In fact, this process could be continued, replacing one neurone at a time. Eventually, the entire brain could be replaced with microelectronics, and there would be no functional difference between the electronic, and the real brain." (Luxion, 1995) This idea, however, does not consider the qualitative contents of brain. As we have seen, the struetural features of a brain are intrinsically joined with its mechanisms of data-elaboration (categorisation mechanisns -Edelmann 1992). Given this, the electronic brain and the 'real' brain cannot be interconnected by a relation of identity-continuity either because a maximal similarity and causal correlatedness r-relation do not link the microelectronic constituents, or because the plasticity of mental phenomena is not a computational process. The 'new' brain, in this sense, will be really similar to the 'natural' one but it cannot be its identity-continuer. 
s If my mind is soltware, I can only experience quantitative changes in my memorised data because my relationship with the world is only a continuous update of external stimulus. Also my reactions toward external stimuli will remain unaltered, and therefore my psychological identity does vary through time - think of a robot.

"Professor Damasio in a recent interview delined this deficiency as follow: "Patients with so called blindsight can accurately point to stimuli presented in their visual field - yet they are unawarc of the presence of those stimuli. When a point of light goes on the lelt visual field, for examplc, the patient is able to point to it accurately. But he will state, quite truthfilly, that he did not see the light. Presented with the same light in the right visual field, the patient will be able to both point accurately and report having seen the light. A similar anomaly occurs in patients who cannot recognise previously familiar faces but can demonstrate through emotional responses, that they discriminate familiar faces from unfamiliar ones." Damasio 1998:88-90

"An example of this direct link between consciousness and neural inputs is the Zeki's experiments on vision mechanisms. The neurologist Zeky addressed the vision mechanisms in monkey's brain. He discovered the existence of differem brain structures specialised in elaboration of visual data (V1, V2, V3, V4, and V5 cerebral areas). His studies, however, did not completely explain the visual duta elaboration mechanisms because, as he underlined, we cannot separate the above mentioned phenomena to the consciousness problem. According to this, the unity and synergy of neural phenomena continue to be an indivisible dilemma

"Why do I have the possibility to choose between two actions (for example "to drink the water or not')? Since my mental software is mechanically one dimensional, how can I choose to drink or not? My intentional capacity ought to contradict the (coherent) software's organisation of my brain. In fact, when a computer receives the cornmand 'print', it only makes what the received command requires.

${ }^{9} 1$ have to point out that the American neurologist has adopted a theortical approach to the Mind problem rather than scientific data that can completely support his theory from an experimental point of view. However, 1 do think that Bdelman's contribution is really important because he formulated an uniffed theory of Mind which takes into account the biological leatures of neuronal systems as well as the theoretical implications they induce (topobiology, neural Darwinism). I am aware that Edelman's theory would need a much deeper analysis but unfortunately this would not allow to the to continue exploring the notion of individuality which is the main subject of this issue.

"Dennell defined this mechanism as a representational system. The Representational System is a system that can adapt itself to the variability of reahity (in Dennett and Hofstadter 1981:189198, Hotistadler 1979).

"The capacity of living matter to self-replicate is reinforced by its capacity to change the replicated structures (e.g. DNA). Crystals can only repeat a structural disposition of atoms. In the first case we have a structural variability (plasticity), in the second, only a structural repetition.

${ }_{12}^{12}$ am thinking of mental states as biological phenomenon. I am not considering Freudian psychology. When $f$ talk of psychology $I$ mean the individual's capacity to operate determinate acts (for instunce, to run).

1. The imnume system is a good example of the individuality of an organism: (1) Each individual has an individual immune system. (2) The immune system bas an internal biological 'memory'. (3) Interaction with its environment determines a plastic change in the immune system. The immunity system is an example of biological memory because immune cells have the capacily to memorise the Self (the organism) and to recognise the non-Self - antigens. The 
immune system is a clear example of an organism's plastic variability depending on environmental factors. (Lewin 1990; Singer and Breg 1991; Old, 1988).

${ }^{14}$ I am not saying that manmals do not have sclfrawareness. I am only saying that their selfawareness is different from the one of those organisms that have the capacity to create reflexive self-references, and to translate them into the indexical expression 'I.' In this sense, I believe, when we consider life forms having a primary consciousness, we have another level of selfawareness: the capacity to refer itself as a determinate time and to link this mechanism to the past.

${ }^{15}$ It is not easy to explain the role of memory in secondary consciousness. Even if we try to analyse the peculiarities of secondary consciousness via neurophysiology we will not have access to the content of mental events from inside. Wc can thus approach the modalities of neural phenomena, but not their qualitative features.

${ }^{10}$ Broca's area and Wernicke's area connect the cerebral areas that receive and analyse noise to other ones that determine conceptualisations and movements. This mechanism is bipolar -the neural impulses can run in both directions.

${ }^{17}$ A different explanation of linguistic processes is Chomsky's theory (1980). Chomsky wrote that the syntax of a language is completely independent of semantic, linguistic, and cognitive phenomena. Donaldson (1978) has criticised this position. He demonstrated that children's capacity to learn semantic mechanisms relied upon their expcricnces. This means that the first condition of linguistic systems is the individual-environment relation. I substantially agree with this vicw.

${ }^{1 *}$ The literature of this cognitive process is vast. See: Butterworth 1992, Kagan 1981, Fogel, 1993. Pinker 1999, Rochat 1995

${ }^{19}$ If we think of the immune system we have a clear example of how the interaction between Self and non-Self is a basic mechanism of an individual's cxistence. Chernyak and Tauber explained this meachanism as follow: "(...) Self-determination has two aspects. First, the immune system assumes the responsibility from providing itself with its own. Other; the system determines that is its other. Delinition of the Other is not the business of an external observer, contemplating what originated outside the system, but it is the business of the system itself. Thus, the definition of the Other is complementary to the definition of Self. Second, Self is the self-detining (in a sense, self-creating self). But contrary to the autopietic interpretation, we believe this means that the Sell does not discover itself as a ready-made presupposition, but creates itself upon the "horizon" of the Other. The Other as positively defined within Self, is the Self's chisel for forming the Self. From this point of view, integrity of an organism does not coincide with its identity. If we reserve the word "Self" tor integrity, then the "Self" is complementary of two reflections. Sell as a "finction" of the Other, and the Other as a "function" of the Sclf. Thus, self-identity (the second aspect) is only one of two complementary (self-) definition of Self." Chernyak and Tauber, 1992:148 Clearly the concept of "function" between Self and Other involves the influence of the Other in the Self-synthesis, otherwise the Self should be an immobile system, self-integrated in itself, and completely independent of external factors. However, we have to notice that the function of the other also needs a Self's function of itself. The Self is the function of the other, but this function is finalised to reinforce the Self-synthesis from its inside, and not to make the Self completely contingent on the Other. This is because the Self is dialectically self-referring to itself - autopoiesis - and self-referring to the Other. The correct view that explains the Self-synthesis, I believe, is the co-existence of both these self-synthesis mechanisms. 
21"Let us deepen now our real topic: the role of memory in the synthesis of the Self. When an organism has its pereeptual inputs, the complexity of its neural structure increases. The interconnection among neural maps cause extensive connections within brain circuits, and increases the associative property of mind. The increase of cognitive stimulus increases the brain complexity and the possibility to have complex (rational) replies to external reality. The relationship between organism and reality influences the neural structure of the individual's brain. The functionality of the central nervous system refers to the organism's mnemonic capacity. In fact, if a subject were deprived of its capacity to memorise its past experiences how could the mind have a fitness value?

${ }^{21}$ The first remark to Locke's criterion refers to its inferential nature. Butler (1975) pointed out that the criterion proposed by Locke is circular, since the concept of memory presupposes the concept of personal persistence. If we follow Locke's criterion we will hypothesise that a continuous and constant relationship exists linking my Selves over time. But if an individual is comatose or has irreversible amnesia, how can we think of their identity as a continuity? Locke's claim would induce us to conclude that his identity-persistence is interrupted. Then, 'the eriteria for persistence of persons are not the same as the criteria for the identily of tiving human bodies' (Brock Sides 1997). This example shows that thinking of an identity view in Locke's perspective means disturbing the relationship between the present and past constitution of our Self. If I attribute to my past the role of first reference in self-reference, I need to maintain a fluid continuity in my psychological identity through time. Those problem cases in which my psychological identity is internupted (brainwashing is an example) causes Lockean theory to collapse.

${ }^{22}$ On the context dependence of 'intelligence' see Richardson (1999). The environmental factors influencing people's IQ are analysed in Wilckelgren (1999: 1832-1834), an analysis on the relationship between intelligence and environmental complexity is (iodfrey-Smith and Ruse (1998).

${ }^{23}$ As we influence reality and our memory, in the same way our continuity is a mutable process that maintains a logic but that varies in its modalities and contents. I can have a psychological continuity because of a "feeling", without basing this process on memory. Nevertheless, identity will continuc. In realising this, we replace a view of the subject as a passive container of experiences, with one that considers the subject an active agent of experiences. The self influences either its relationship with reality or its relationship with a recognised past. The memory is a property of our self awareness, but is only a tool to perceive our awareness of our exjstence (Wilkers 1988, Varela Thopson and Rosch 1991). The eoherence of this act refers to the process through which the self bases its reflexive self-references. The internal coherence of our psychology will thus be connected to the relationship within our past selves, but also (and more fundamentally) to our self-reflexiye properties.

${ }^{24}$ The awareness of my presence as a determinate subject is subordinated to my reflexive selfreterence. My psychological identity thus refers to the consciousness that the person who possesses 'that' psychology is 'me'. If this step is not taken, I am not aware that I am the subject of my 'experiences'. Even if we say we have to investigate the r-relation that links my multiple selves, I need to know that these selves are me and that I am indeed a self. When I am deprived of my internal refesence, 1 become the spectator of the existence of another subject who is me though I do not know him. In this case, the reference from-the-inside ought to become an outside-reference. The self-awareness processes constitute the substratum on which the psychological identity is build. 


\begin{abstract}
${ }^{25}$ It should do so by emphasising the unifying processes of our mental entity over time. This metric will be reinforced by the relationship within our selves and unified by a self-awareness process that is not nccessarily a rational one. If a sell is constituted as a variable self-reflexive dimension (Parlit 1984, Nozick 1981), this proecss absorbs into itself either our existing self or our past selves. My self-reference is connected to my temporal connotations. When the rrelation links my memories to myself, and when this process is enriched by my self awareness, I have consciousness of my historical existence. The role of my reflexive self-reference is to unify my different selves in a unitary entity that is me, and which is part of my existence (Dennett 1991, Lyson 1986). The whole of mental entities is organised by a causal relationship within my present and past self-relerences. At the same time this unity is determined by the self-reflexive mechanisms that allow me to be conscious of my existence as a Self and as an entity that continues.

${ }^{26}$ Hume (1784) emphasised that human beings can only have the impression of the existence of their Self, because all the questions of their Self appertain to the Self from-the-inside (see: Penelum 1975; Mclntrye 1989: 547-557; Montaleone 1989, Lecaldano 1991). This means that the value of psychological identity in the global identity processes is weaker than it previously was. Or ever better, the heart of the identity problem is focused on the internal relationship that causally links our mental existence through time. When Hume said that the Self is not an abstract requirement of our existence he meant we have to think of ourselves as variable psychological structures. This variability of Self is not only a constitutive variation (my memories are different) but it is also a qualitative transformation which influences either my present Self or my relationship to my past memories. According to this, it is not important to find a fixed and determinate property explaining our psychological continuity, because all of our efforts are destined to arrive at the same conclusion; the Self is a variable dimension. Thinking of ourselves as a unitary entily is only an illusion. When we say that our unitary Selr is an illusion accomplished by our intuition of its existence, we explain our life (the milestone of my life is 'I am Maurizio, and not another person') by destroying the sureness of our presence and continuity as a determinate entity. If we consider the variability of a Self, the problem of the internal connection between the 'different faces of our Self' becomes the core of our questions on the nature and meaning of our existence as individuals.

${ }^{27}$ Locke, J. Essoy concerning Human Understanding, 1931, Cambridge University Press, Cambridgc, eds. Benjamin Rand.
\end{abstract}




\title{
I.7 The Organic Unity Theory, and the Variability of Our Existence
}

\author{
"The debate until the 1970 s assumed that identity, an all-or- \\ nothing one-to-one relation to use when thinking about \\ personal survival. For a person $A$ at time 1 , and a person $B$ \\ at time 2 , either $A$ is the same person as $B$, or $A$ is not the \\ same person as $B$. There can be no degrees of survival. But \\ thanks to the work of Derck Parfit (1971. .11, 1984) it \\ became casier to envisage degrees of survival. On Parfit's \\ view, as that of Locke, Lewis and Nozick, one's identity is \\ constituted by one's psychological profile. Parfit's radical \\ departure from their theorics is that we should dispense \\ with the concept of identity, and instead should talk in \\ terms of personal survival over timc. What matters as time \\ goes by is not identity, but the continuity and connectedness \\ ol one's psychological profile... Instead of identity we \\ should concern ourselves with the direct psychological \\ connectedness and continuity of psychological properties" \\ (Perring 1997:176-177)
}

In the previous chapters I have claimed that: (1) the organic unity view is an appropriate model to explain the individuality of an organism; (2) two parallel systems unify organisms: (a) biological cohesiveness (unification of a body), (b) reflexive self-reference (unification of a Self); (3) identity is a logical process of stability in the midst of variability. In this chapter, I will explore the problem of individuality through modern philosophy. I will not repeat what I have already said about the organic unity model. What I will add here is an analysis around the persistence of an individual as a determined identity'. My basic problem, then, is; 'why does identity of individuals persist over time? To what extent can we say that an individual persists while varying?

I will explore the above-mentioned problems by considering as my conceptual platform some statements that have reached an almost general consensus among philosophers: 1) We cannot think of identity-persistence as a maintenance of a determined quantity of bodily-psychological traits; 2) We cannot think of identity-persistence as an all-or-nothing mechanisn; 3) We cannot think of identity as located in a Cartesian entity. As the reasons which induced me to this choice have already been explained in the previous chapters of this work (chapter $1 ; 2$ ), I will not systematically analyse the notion of individuality in philosophy (see, inter alia, Parfit 1984 , 
Nozick 1981, Olson 1997, Di Francesco 1999), but rather that of personal survival over time instead.

\subsection{Persistence of Individuals}

Why does an individual persist over time? When I see a picture of me at the age of $10, I$ recognise myself now as the continuer of the boy then in the picture ${ }^{2}$. Why? My psychological traits, my biological constituents as well as my life are completely changed. Nevertheless I know I am 'that' boy 22 years later. I recognise some of his features in myself. Perhaps some morphological similarity, some psychological continuity, or simply 1 remember when the picture was taken. Can I say that the persistence of my identity refers to a bodily (Williams 1973, Nagel 1973) or a psychological continuity (Parfit 1984, Nozick 1981)? Is individuality-persistence dependent on some biological or psychological features of an individual?

When we wonder about the persistence of individuals over time, it is extremely hard to say whether a specific element, or a quantity of this factor (say $50 \%$ of my memories), can safeguard subjects' continuity. This is true for the continuity of an individual at both long (say 20 years) and short time frames (say 24 hours). As Parfit said, it is hard to remember $50 \%$ of the actions we did yesterday ${ }^{3}$ (Parfit 1984, XI: 81; Kolak and Martin 1987, 1991). Then, a conceptual difference between day-person persistence (a person through a single day) and Life-person persistence (a person through a whole Life) does not exist (Parfit 1984). Living beings continuously vary. Biological variability and psychological continuity are both consequences of the historical nature of our existence, our biography (Rachels 1996, Lecaldano 1999). As we vary within a short time frame, our variability at different times (20 years) merely reinforces the degree of dissimilarity among our selves, while not affecting our continuity. And this is true for both our bodily and psychological features. The only difference between day-person and Life-person persistence is the growth of dissimilarity rates in the succession of tokens of person-phases. The foregoing leads to, what may be called, the paradox of identity or the puzzle of identity. On the one hand the persistence of individuals is a basic element to take into account when we explore the notion of individuality in organic systems, while on the other hand when we attempt to fix a border line in personal persistence, we deny the diachronicity of living beings and we see identity as a 'all or nothing process ${ }^{4}$. Both these claims are biologically false (see previous chapters) and philosophically debatable. Philosophers tend to approach 
identity puzzles looking at individuality as a plastic and variable process (Nagel 1974, Williams 1973, Parfit 1984; Nozick 1981, Dennett 1978, 1981, Olson 1997, Wiggins 1980, 1987) in which a causal correlatedness links an individual to his/her continuer over time ${ }^{5}$. The nature of this connection is, at this stage, an open question.

As I have said, a number of philosophers have dealt with subjects' continuity. A subject was seen as a variable entity determined by the cohabitation of bodily and mental elements. Both the individual psychology (psychological traits) and his bodily identity vary. Does the subject's variability invalidate the concept of personal identity? Can we defend a view that respects the variability of individuals while respecting their diachronic unitary existence? We have two different responses to these puzzles; 1) identity is not important; 2) identity is important but it should be seen as a variable process. Derek Parfit (1984) has advocated the first one; Robert Nozick (1981), inter alia, the second one. I will analyse both of them and I will defend the thesis that the second one is adequate to explain the existence of individuals as biological and psychological entities. The box below summarises both criteria of identity/continuity/survival:

\begin{tabular}{|c|c|}
\hline Parfït's Criterion & Nozick's Cri \\
\hline $\begin{array}{l}\text { nportant because what is important is } \\
\text { the r-relation, the psychological } \\
\text { onnectedness and/or the contimuity } \\
\text { ith the right type of cause (every } \\
\text { ause can be the right cause). The } \\
\text { indamental clement for the continuity } \\
\text { I a subject }(X) \text { in some other (Y) or } \\
\text { i himself - is not the identity of the }\end{array}$ & $\begin{array}{l}\text { The identity of an individual is important. } \\
\text { "Something at } t 2 \text { is the same entity as } X \text { at } t 1 \text { if } \\
\text { it is } X \text { 's closest continuer, is a close enough } \\
\text { contimuer and is enough closer than any other } \\
\text { continuer. (...) To say that something is a } \\
\text { continuer of } X \text { is not merely to say its properties } \\
\text { are qualitatively the same as the } X \text { 's, or } \\
\text { resemble them. Rather it is to say they grow out } \\
\text { of X's properties, are calusally produced by } \\
\text { them, are to be explained by X's earlier having }\end{array}$ \\
\hline
\end{tabular}

\subsection{Psychological Continuity}

As I have previously said, Parfit addresses the problem of individuality by stressing the individual's psychological identity. This view (called "psychological criterion') refers to three conditions: "There is a psychological continuity if, and only if, there are overlapping connected chains. $X$ today is one and the same person as $Y$ at some past time if and only if: (1) $\mathrm{X}$ is psychologically continuous with $\mathrm{Y},(2)$ this continuity has the right kind of cause, and (3) there does not exist a different person who is 
also psychologically continuous with $Y "$ (Parfit 1984:207). This claim harks back to the philosophy of Locke and Hume. Both these philosophers, although they advocate different theories on individuality (Di Francesco 1999), say that what is important to be an individual, is to posses psychological traits. Parfit considers this statement as the conceptual platform of his philosophy. He claims that wondering about the subject's continuity means exploring the subject's psychological persistence. The variability of this element induces him to say that identity is not important. (Parfit 1984). According to Parfit, when we realise that we cannot think of individuals as Cartesian entities (Clark 1991; Lakoff 1987; Searle 1992; Minsky 1986; Churchland 1986, 1988, Olson 1996) and that personal identity is a mechanism of bodily and psychological variability, then the causal link between $r$-related selves at different times that constitute our diachronic existence becomes the basic element to take into account in identity puzzles. The causal relatedness between subject's selves does not refer to a specific logic of continuity but is a variable process ("every cause can be the right cause"). Then our attempts to fix a metric of continuity is external to the investigated object (outside reference) and the relationship linking two entities in terms of continuity varies according to their features and their causal relatedness. This continuity is a transitive property of an individual.

To summarise, Parfit considers an individual as a diachronic entity that varies so radically that the very concept of identity becomes implausible. According to Parfit, when we wonder about people's identity we should avoid thinking of their existence as determined individuals, but we should focus on the psychological relatedness that safeguards the subject's continuity over time.

If we apply Parfit's philosophy to my previous example (my continuity in the boy in the picture (Y)) I can say that I continue his identity when there is a link between $Y$ and I $(M)$ that makes the relation $Y-M$ causal. It does not matter if this link refers to my memories or other factors. What is important is that $Y$ and $M$ are causally related regardless of the nature of this link ${ }^{6}$. The problems arising are: To what extent can the variable nature of our being weaken individuals' identity (Dennett 1987 , 1991; Nooan 1989:229-231, More 1996)? According to Parfit, we should answer this question by saying that the diachronicity of our existence should induce us not to think of our identity as the basic question of our existence. We should focus on the logic of continuity through which we continue to exist as individuals. This claim is extremely controversial. The idea that our 
identity is not important has a number of contraintuitive factors. I know (intuitively) that $I$ am a Self. And around this awareness, I live. In the light of this, if personal continuity is a variable process, why should we deny individuality? Is the problem of people's continuity so complex to make identity unimportant?

These questions, to me, refer to a misunderstanding of Parfit's view. It should be noted that Parfit does not deny the individual's identity, he simply says that this element is not so important in the course of our Life. The first consequence of his theory is that we should avoid thinking of identity as a static dimension (one-to-one relation) but we should look at our existence as a fluid process. Personally, I have no problem in accepting this idea. My organic unity model has offered similar conclusions. In a biological perspective we have no reason even to think of being able of minutely describe our presence as a determined dimension. The historical nature of living beings does not allow us to think of identity as a static process. Both the biological and psychological features of living beings refer to a cascade of related events determining the subject's features at a determined time and through time. These constituents are causally interrelated ${ }^{7}$. It does not matter if we think of mental traits or bodily features as determinants to explain living beings' individuality. Both our biological and psychological biography determines our existence and our persistence. The only factor that may induce us to see identity as a static process is the claim that individuals are Cartesian entities. However, as I have already said, we have no reason to defend this view (see Parfit 1984).

Thus, I can easily accept the concept of individuality in terms of diachronicity (Lewis 1978, Robison 1985, Mills 1993, Noonan 1989). To me, what is debatable in Parfit's view is, on the one hand the predominant weight he attributes to the subject's psychological identity, and on the other hand the difficulty to use his theory to explain the individuality of those subjects who have a psychological discontinuity due to some pathologic dysfunctions (Alzheimer patients or subjects deprived of a psychological identity, e.g. anencephalics, comatoses).

As to the first criticism, it is clear that when Parfit stresses the need to have psychological continuity in the individual's diachronic existence, he considers the psychological features of an individual as the main elements to take into account in identity dilemmas (Robinson 1985, Mills 1993). His criticisms of the Lockian (quasi memories), Cartesian (the reincarnation problem) views as well as the case studies he analyses (brain transplant, teletransporter etc.) de-emphasise the value of bodily identity and privilege 
the psychological identity. Then his theory on individuality refers to the subject's psychology rather than his biological features. Clearly when an individual does not have psychological traits (anencephalics, for example), or his/her psychological continuity is radically changed (comatoses, amnestics, for example), then it is not easy to think of Parfit's theory as an explanatory model to use to describe the individuality of such organisms.

However, as Olson underlined (1997), practical case studies (e.g. anencephalics, pre-embryos) show that psychological continuity is not the only explanation of the existence of living beings as biological entities. Those cases in which an individual does not even have mental traits should induce us to approach the problem of individuality by considering the biological features of individuals as well (Olson 1997:16-17, Di Francesco 1998:262-273). In the light of this, which is the approach I have used in this dissertation, even if Parfit's theory is extremely relevant, when we look for a view that could explain individuality of living beings, we need a theory that on the one hand would be applicable to different life stages of the individual existence (from embryo -proto-consciousness- to adults - rational subjects), and on the other hand would be capable of explaining both their psychological and bodily continuity. In cases of amnesia, or brain damage, psychological continuity of an individual may be radically changed and may induce deep permanent changes in his/her personality (Olson 1997). Then, the causal correlatedness between individual's diachronic Selves becomes a 'not completely adequate' tool to describe the continuity of a subject.

Parfit says that, although an organism varies qualitatively and structurally, the r-relation linking a subject to his continuers should maintain a state of cohesiveness causing an uninterrupted stream of consciousness and biological cohesiveness. Nevertheless, the subject's stream of consciousness may also be interrupted or profoundly changed ${ }^{8}$ (brainwash, permanent amnesia, Alzheimer, head transplant). Also the biological cohesiveness can be radically affected by factors that do not belong to the subject (transplants ). To what extent can identity-persistence be causal? Are we able to deal with the problem of individuality taking into account both the subject's body and self'?

\subsection{Closest Continuers}

Robert Nozick (1981) also dealt with identity puzzles. He claims individuals to be heterogeneous and complex self-aware entities whose 
biologica1/psychological complexity should not be made dependent on some specific bodily/psychological elements (the brain -Nagel 1973, the body Williams 1973, the self -Unger 1993, Robinson 1985, Mills 1993, and so on) or should not be approached through rigid criteria of classification. Nozick in fact says that any attempt to make the individuality of an organism dependent on a specific factor of his/her biological existence is the result of a projection of an observer who conceptualises the individuality of others from the outside. This process refers to the weightings system used by observers and not to the individual from his/her inside. In the light of this, he (a) uses the organic unity concept to explain the notion of identity; (b) stresses the basic role of self-awareness in individuals' Self-synthesis; (c) claims a metric of continuity (the closest continuer model) in identitypersistence problems. Let us first say a few words about Nozick's identity theory and secondly about the closest continuer model. (Clearly, here I will not explore the organic unity concept because it has already been analysed in the first two chapters of this dissertation.)

Nozick says that human beings are individuals because they have a reflexive self-reference ${ }^{10}$. This subjective capacity on the one hand obstructs the use of outside references in classification processes, and on the other hand stresses the basic value of subjects' intellectual capacities towards personal identity problems. Those entities that have a reflexive selfreference, a self-awareness, have a higher degree of organic unity compared to other living beings that do not reach a sufficient level of complexity to be (autonomous self-aware) individuals. The brain complexity allowing mental capacities, then, ranks different organic systems in Nature. Human beings, as self-aware entities have a primary position in natural taxonomy. Their value as individuals is dependent on Self-synthesis.

Nozick, then emphasises the central role of Self-synthesis in identity dilemmas. The idea is that through Self-synthesis we become aware of our existence as determined and variable entities (ibid. 71-104). This mechanism pertains to the reflexive self-reference property of individuals according to which an organism synthesises itself via internal references. The awareness of a subjective existence, then, refers to the weightings of dimensions that each individual defines during his/her self-referential processes" .

In other words, according to Nozick, we know ourselves as determined individuals with particular features whose weight is attributed by us according to our scale of values ${ }^{12}$. (For example, a Playboy model might 
privilege the continuity with her/his bodily requirements. While another person (e.g. Einstein) might attribute a higher weight to the continuity of his/her intellectual capabilities.) The measure by which we synthesise ourselves cannot apply to other individuals.

The relativity of the weightings of dimensions in identity puzzles pushes Nozick to approach identity problems in relation to the maximum elasticity of subjects' transformations. He defends a schema that fixes a metric of continuity in identity dilemma: the Closest Continuer Schema (CCS).

\footnotetext{
"The closest continuer view presents a necessary condition for identity; something at $t 2$ is not the same entity as $x$ at $t 1$ if it is not $x$ 's closest continuer. The 'closest' means closer than all others; if two things at $t 2$ tie in closeness to $x$ at $t l$, then neither is the same entity as $x$. However, something may be the closest continuer of $x$ without being close enough to it to be $x$. How close something must be to $x$ to be $x$, it appears, depends on the kind of entity $x$ is, as do the dimensions along which closeness is measured (...) The Closest Continuer view holds $y$ at $t 2$ is the same person as $x$ at $t 1$ only if, $y$ 's properties at $t 2$ stem from, grow out of, are causally dependent on $x$ 's properties at $t l$ and, there is no other $z$ at $t 2$ stands in a closer (or as close) relationship to $x$ at $t 1$ than $y$ at $t 2$ does." (Nozick 1981:34-36)
}

Nozick bases his continuity criterion on arguments from perception to intuition. The essential idea of this argument is that we perceive the continuation of objects according to a certain schema; which he describes in $\mathrm{CCS}^{13}$.

He describes an experiment of a psychologist (Shimon Ullman) that intends to establish how people classify discontinuous appearances, when two appearances of the same object switches to the appearance of two different objects $^{14}$. Since the participants recognise the object continuity as depending on the appearance of its qualities' continuity, Nozick concludes that: " $(. .$.$) a later object Y$ is the same as an earlier object $X$ only if $Y$ 's properties grow out of, are causally dependent on $X$ 's properties at the earlier time; (...) there is no other $Z$ at the latter time that stands in a closer (or as close) relationship to $X$ than does $Y$ "' (Nozick, ibid. p. 36-37)

The basic idea of Nozick was the need to define a metric of continuity of identity-persistence. According to Nozick, the two main elements to take into account in identity continuity are (a) maximum similarity among constituents of objects as well as (b) causal relatedness between the above mentioned constituents ${ }^{15}$. This means that properties of objects (at different time frames) must be related. We can say that an identity of an object persists over time when maximum similarity and causal 
relatedness are fulfilled. It does not matter whether we apply these continuity conditions to some specific features and not others, what is important is that the continuity logic remains coherent. Wondering about the continuity of an entity, according to Nozick, should induce us to apply CCS to those features that are significant for the identity at the time of the classification. This means that when, say, we wonder on the individual persistence of embryos at different time-frames (10 days $-\mathrm{x}$ at $\mathrm{t} 1-$ and 2 months $-y$ at 2 ) we can explore the continuity of $x$ in $y$ by taking into account those elements which belong to the embryo's physiology at that time. It would be impossible to make the continuity between $x$ and $y$ contingent on some psychological factor because we have no information on the proto-consciousness of pre-embryos. Even if the neurosciences tell us that a proto-consciousness exists at that stage of embryogenesis (Ossicini, Mancini 1999), we cannot say that between $x$ and $y$ an uninterrupted stream of consciousness remains since proto-consciousness of a pre embryo, which is antecedent to cell differentiation, and the embryo's consciousness, which is subsequent to neural cells differentiation, cannot be equated. However, according to Nozick's view we may apply CCS to other constituents, for example embryo cells. In this case, the 'maximum similarity' condition would be applicable to genes, for example. The 'causal relatedness' condition would be applicable to meiotic processes and cell differentiation. This means that even in those cases in which individuals' identity is problematic we can look for appropriate features/properties of individuals and explore their continuity through them. Other problem cases in which a subject's stream of consciousness does not coherently continue through time (Alzheimer people, e.g.) can be approached via other causal relatedness factors. Then the main advantage of CCS is its flexibility. CCS is applicable to all features, properties and constituents of an organism. We can select those ones that are more significant for the biological nature of the investigated object at the time of our classification. We can also analyse the subject's psychological continuity via $\mathrm{CCS}^{16}$. What we must do is simply explore object's continuity through the system we have used at different times $(t 1, t 2)$. This coherent approach will guarantee an analysis that, while being done externally (we wonder about the object's persistence from the outside), we can look at identity-persistence as a coherent evolution of an entity at different time frames.

At this point, one might object that Nozick defends a contradictory view on individuality. In fact, on the one hand he says that CCS should not be applied to some specific components of an individual. On the other hand, 
he claims that the psychological criterion has a basic value in identity puzzles. Nevertheless, we must be aware of the distinction Nozick made between individuality as a logic process through which a whole became a unitary entity, an organic unity, and the concept of an individual as applicable to human beings. The first analysis refers to the individuality of all things. The second one explores 'the reasons why' human beings are different entities compared to other physical systems (individuals as selfaware entities). My use of Nozick's philosophy strictly refers to the first approach and intends to show that the second one would not completely explain a number of problematic case studies. Let me explain why the psychological criterion cannot completely explain individuals' existence.

I agree with both Nozick and Parfit that the psychological criterion is extremely important in identity puzzles ${ }^{17}$. What sets my position apart from the ones of the above mentioned philosophers is the value attributed to this factor. I do not think we can consider it as a prerequisite for individuality. As I have already said with regard Parfit's view, practical cases exist in which organisms do not have a unified Self. However, such entities exist. Then, when we deal with the notion of individuality we need first to explain the biological nature of the investigated objects and subsequently improve this study with other factors (such as subject's psychology) which are significant to describe subjects' features but which cannot be seen as pre-requisite to consider organisms as individuals. This means that we have to look for a theory of individuality that is applicable to both the micro and macro complexity of organic systems. We need to explore the notion of individuality by avoiding the use of rigid classification criteria that are external to the objects and context-dependent ${ }^{18}$. Rationality, in this sense, cannot be considered as a precondition of individuality because (a) living beings are complex (biological) entities whose features cannot be ranked externally by a rigid classification system (in this case the basic value of the psychological criterion), (b) a theory of individuality should be coherently applicable to living beings according to their individual-specific and species-specific features, (c) subjects' complexity and variability should be taken into account in identity theory. Human beings are biological entities, as such a theory on individuality cannot forget their physical matrix (O1son 1997, Inwagen 1990). Otherwise we risk advocating a theory which is unable to explain the existence of individuals through time (let us think of embryogenesis), the existence of a number of concrete and real problem cases in which people loose psychological continuity (comatoses, amnestics, multiple personality patients, and so on), 
the dual nature of living beings (to be a body and a self) or the individuality of different living beings other than (healthy) human beings.

The organic unity concept, on the one hand, and CCS, on the other, can help us in exploring the notion of individuality, its heterogeneous complexity and diachronicity. In the light of this, I do think Nozick's theory can provide us with better tools to study living beings' individuality. Even if Nozick did not deal with this problem within theoretical biology. As I have said, he considered it as a classification problem and subsequently as a problem for the individuality of human beings. In this dissertation, I have completely changed this approach. Individuality has been thought of as a problem for all Life forms.

\subsection{Conclusion: Diachronicity and Complexity of Individuals.}

Both Nozick and Parfit hold theories of individuality that completely revolutionised the way in which philosophers addressed individuality. Identity finished up by being considered as a static process and became a fluid process of stability-in-variability. The central idea they introduced was that "(...) Instead of identity we should concern ourselves with the direct psychological connectedness and continuity of psychological properties" (Perring 1997:176-177). However, in this chapter I have underlined that such an approach cannot minutely describe the existence of individuais. On the contrary we should use a theory able to explain the biological nature of living beings, as physical systems, and subsequently approach their phenomenological nature.

However, when we realise individuality to be a diachronic process, it becomes important to fix a metric of continuity for personal survival. Nozick's CCS, in this sense, is an extremely useful explanatory tool to explore identity-persistence puzzles. This is because it can be applied to those features of organisms that have a significant role 'for' their existence at different times. As such, it can respect the heterogeneous complexity of living beings, as well as the coherency of identity continuity. It can be used to explain both the biological nature of living beings (biological variability) and the psychological nature of diachronic selves. As such, it fits better into questions related to biological individuality. 


\section{NOTES}

1 This dissertation claimed that the concept of individuality should avoid metaphysical explanations, because they cannot deal with the plyssical nature of living beings. This is evident in biology and neuroscience. However, both the dialectical nature of individuals and the complexity of mental phenomena have induced some philosophers to re-defend (pseudo) dualistic claims (Eccles 1989, Popper \& Eccles 1977, Swinburne 1984, Chomsky 1980). This thesis was strongly criticised by countless philosophers and scientists (inter alia: Searle 1992, 1997, Lakoff 1987, Jonson 1967, Parfit 1984, Edelman 1992, Dennett 1995). I will not repeat what they have already said on the limits of dualism. I will use a reductionist approach to the identity problem.

${ }^{2}$ If I think of myself 19 years ago, $I$ can recognise that there is an internal relationship between the juvenile Maurizio in, lets say 1977 and Maurizio in 1996. The 'link' that joins different life-stages of my existence contains my identity-continuity. My identity persistence may be understood as reducible to certain other facts about bodily psychological connectedness and continuity. In this sense, I am not something ontologically separable from the relation with my past (and future) selves (R-relation). We can define the R-relation in a variety of ways. For example, I can think of $R$ as the continuity of my body, as the continuity of my memories, or as my relationship with other people, and so on. If "Maurizio at the time of 1977" is $\mathrm{M}$, and "Maurizio in 1996" is Mx, then the reiationship M-Mx admits only onc direction of persistence $\mathrm{R}$ links $\mathrm{M}$ to $\mathrm{Mx}$.

3"I don't remember many of the experiences that I had at a time exactly a week before this one. This fact points to a deeper problem. The relation of strong-connectedness of quasi-memory is not a transitive relation, whereas the relation "is the same person as" is supposed to be a transitive one". (Side 1997)

${ }^{4}$ "We cannot defensibly believe that our identity involves a further fact unless we also believe that we are separately existing entities, distinct from our brains and bodies. We cannot defensibly believe that our identity must be determined, unless we believe that the existence of these separate entitics must be all-or-nothing ... If personal identity does not involve a further fact, it is very hard to believe that there can be such a [border] line. This is even less plausible than the Reductionist View " (Parlii 1984:240)

$s$ "The fundamental element for the continuity of a subject $(X)$ in some other $(Y)$ - or in himself - is not the identity of the same subject, but the r-relation which links $X$ and $Y "$ "(Parfit 1984); and "To say that something is a continuer of $\mathrm{X}$ is not merely to say its properties are qualitativcly the same as the X's, or resemble them. Rather it is to say they grow out of X's properties, are causally produced by them, are to be explained by X's earlier having had its properties, and so forth"(Nozick 1981).

'An alternative solution to Parfit's view is the one of Kolak and Martin (Kolak and Martin 1991). They suppose a strange siluation: the entire universe as we know it flickers on and off every instant. Between instants, some sort of cosmic randomizing device creates an infinite number of sequences and then selects and conjoins with the others only those which correspond in a normal way to the previous instant. In that case, what exists at any one instant did not causally arise out of what existed at the previous instant. It is not likely, to say the least, that science will discover this. Would it make a difference to our concept of personal identity, or would we go on just as before? "We believe that we would go on just as before, and that the laet that we would casts doubt on the causal condition" (Kolak and Martin p.344). This example supposes existencc-persistence without a causal condition. However, since our 
existence as determined temporal selves is strongly based on our lived condition, how can we deprive ourselves of a causal continuity to our existence over time? How can we deny the interrelationship straggling from our past to future selves? Can 1 think of mysclf as an entity that is non-causally connoted? All these objections induce me to reject Kolak and Martin's view.

${ }^{7}$ Let us consider the definition of organic structures of Kalmykov: "The organic structures strucures which are formed by fixation of free envitonmental energy in their structure (c.g. organic molecules) and, as a result, they are able to do some work. Compatibility of these structutes is structurally homomorphic in character, i.e, they have a fundamental unity of specific morphological arrangement. In consequence, this eases their interaction encouraging the possibility of reciprocal transformations." (Vyacheslav L. Kalmykov 1999)

" "Split brain" people (Gazzaninga 1967, 1970) have a psychological identity (Nagel 1971, Baille 1993, Brooks 1994), as well as multiple dissociated personalities patients (Lizza 1993, Braude 1995, 1996). Similar considerations can be taken for anencephalics, embryos, amnesties and comatose patients (Noonan 1989, 1993, Olson 1997).

"Let us consider an extremely controversial case study: the transplant of the complete head. Dr. White has already done a transplant of the complete head between two monkeys (1999). Animals survived. This has demonstrated that head transplant is feasible (technical problems refer to the connection of nerves with the spinal cord). Should we say that in this case the physical continuty of the brain and the (supposcd) uninternupted stream of consciousness would cause the continuity of an individual in another body? See 'Body Polities' in New Scientist, October 1999:48-51

10 "To be an I, a self, is to have the capacity for reflexive self-reference. Something $X$ which could refer, even to $X$, but not retlcxively, is not an 1, not a self." (Nozick 1981:78)

${ }^{11}$ Any attempt to think of the identity of another individual is a projection of observers. They imagine themselves in the analysed identity problem situation. This overlap does not explain the subject's identity from within the subject and makes his/her identity dependent on the perception of its observer.

12 Another philosopher who stressed the importance of reflexive self-references in identity dilemma was Evans (1982). He considered subjects as fulcrum of self-synthesis processes. He distinguished the I-thoughts (based on the indexical reflexive ' $I$ ') from the 'public space' (the subject's experiencing capacity). The first one grounds the second one. The relationships between the subject as autonomous self-referring entity and reality have been analysed by Strawson (1966) and Nagel (1986).

${ }^{1,3}$ The Closest Continuter theory contrasts Partit's theory in its description of the condition needed for transitivity of identity. Parfil's psychological criterion says, "if a person $(X$ at $t 1)$ is R-related to both $Y$ and $Z$ at some later time ( $(2)$, then, $X$ cannol be identical to either $Y$ or $Z$ even if, at a still later time ( $t 3$ ) there is only one person R-related to $X$." Nozick's view states that person $Z$ could be identical to $X$ despite there having been a period in which both $Y$ and $Z$ were continuers of $X$.

${ }^{1+}$ The experiment aimed at studying the mechanism of identity ascription to object's discontinuous appearances. "An object was shown moving in a staight line towards a screen and then disappearing behind it. After a short time, another object canc out of the other end of the screen. The angle of exit was varied, as well the object's color, shape, and velocity. The results of the experiments fitted the closest continuer hypothesis. For example, if the object exited at a different angle, or atter a delay, people assumed that it had been stuck behind the screen and was the same object. But il two objects exited the screen, the one with the angle, 
velocity, so on, of the entering objcct was thought to be the original object." (More 1996, p. 14. 15)

is See my analysis on classification criteria and clustering processes in the first chapter of this dissertation.

16 "The self synthesizes itself not only transversely, among things existing only at that time, but also longitudinally so as to include past entities, including past selves which were synthesized. My currently synthesized self includes past self-stages in accordance with a closest continuer and a closest predecessor schema. (..) Reflexive reference from the inside corresponds to and reflects the reflexive synthesising of the self (as synthesising itself)" (Nozick, p. 91).

${ }^{17}$ I agree with Nozick's analysis on self-synthesis and reflexive self-referring mechanisms. When $I$ use the indexical reflexive ' $l$ ' in my 'first-person' predicates, I refer to myself (the synthesised entity based on reflexive self-references). I cannot misidentify myself because the entity ' $\mathrm{I}$ ' is a psychological (self-aware) organic unity. The problem is that Nozick's analysis on self-referring mechanisms does not consider the dual nature of living beings: to be a body and a self. As I have said, when we consider the psychological criterion as the basic requirements of individuals we automatically deny the identity of those organisms which do not have self-referring capacities and those organisms that have lost their self-referential propertics (patients in comas, anencephalic children, and so on -Salvi 1998, Wiggins 1980, Olson 1997). Why should we deny the dual nature of living beings (Wiggins 1980)?

${ }^{18}$ We might also object that the notion of individual is eulture-dependent (Lycan 1996). As such, it can greatly vary according to cultural paradigms or metaphysics. A clear example of this is the debate which is currently taking place among bioethicists on moral implications arising from the use of new reproductive technologies in biomedicine (see chapler II. 3 of this dissertation). 


\section{I.8. Analogous Processes in the Unification of the Organic Unity.}

In this chapter I will analyse the mechanisms unifying the Self as an organic unit comprised of biological and psychological structures. I will start with the body-mind question and argue that psychological and biological identities are based on analogous functions and modalities working together. In order to examine this thesis, I first clarify my use of the concept of analogy, and then explore the relationship between body and mind.

\subsection{What is Analogy?}

The standard sense of analogy can be explained as follows; analogy consists in any mode of reasoning that depends on the suggestion or recognition of a relation of similarity between two objects or sets of objects (Maurer 1972:60-67). It is a measure of comparison and a tool for conceptualisation. The condition of 'relation of similarity' constitutes the prerequisite of an analogous relation. However, in this chapter I will derive the concept of analogy from its philosophical complications and implications. I will use a specific concept of analogy to illustrate my ideas: analogy as a link between two processes presenting an internal similarity in their results.

The conceptual reasons motivating this use of the analogy concept rests on two classification criterions: 1) similarity between two things does not necessarily involve their analogy, and 2) analogy is not restricted to the margin of constitutive similarities between two objects, but can also be extended to their functional similarities. Let us set the stage for our next example: an analogy between a car and a bicycle.

I can say that 'cars and bicycles' have the analogous function 'to transport me', despite their constitutional mechanical dissimilarities. This use of analogy submits 'analoganda' to a reference (the transport) that analogously joins them. Therefore, I need a point of reference, an idea, that allows the comparison I make between the analoganda's functions. When I change the 'analogon's relation' amongst objects, the analogy is destroyed. Nevertheless, the objects" properties/peculiarities do not vary. Analogy is not a constitutional property of the objects to be investigated. It is a hermeneutic action, involving a reference (the analogon's property), the 
analogans (who individuates the analogy), and at least two analogata. An analogy is external to a thing's objectivity, and is referred to by observers and their weighting system ${ }^{1}$. The subject's reference defines the nature and the existence of analogous relations among things. However, this does not mean that the subject influences the existence of the things themselves ${ }^{2}$.

This means that the analogy is based strictly on measurement systems found in its observer. When I say, 'I am the creator of an analogy', I do not say that my action influences reality (Carnap, 1956). The objects' features remain identical to themselves (one rides the bicycle to class even if it is approached via a faulty analogy). Analogy is not an ontological property of a subject, but merely an interaction with perceived phenomenological reality (Putnam, 1988). The nature of the 'objectivity-subjectivity' relation is one of the most controversial topics of modern philosophy (Husserl, Heidegger and Wittgenstein ${ }^{3}$ ), and cognitive psychology (Brewer 1995:291-306, Campbell 1995:29-42; Cowley 1971:678-683; Epstein 1973:111-121). I do not want to focus this chapter on the relationship between the subjective objectification ${ }^{4}$ (Heiddeger's Dasein), and the objective 'mundanization' of things. My analysis aims instead at demonstrating that both the biological and mental features of an individual have an analogous 'role' towards his/her existence-persistence: to unify a plastic heterogeneity of biologicalpsychological factors in a homogenous entity that persists over time.

In the previous chapter we saw that superior organisms with a secondary consciousness use a symbolic memory. They have a mental image of their presence as volitive and determined Selves. This property is connected to the primary consciousness, and reinforced by semantic and linguistic capabilities. The capacity for Self-synthesis is based on the subject's capacity to use the intentional emission ' $l$ '. The semantic use of 'I' refers to an acute and complex secondary consciousness' phenomenon. When subjects talk about their own presence as a determined entity, they have memorised their diachronic existence as subject (for example, organisms with a primary consciousness cannot consider their identity in the future). Self-synthesis implies an internal coherence with our previous existence.

The consciousness of our presence is the basis of Self-synthesis despite the internal links within our memories varying both qualitatively and quantitatively. It is linguistically and symbolically translated and constitutes the substratum in which we induce our volitive ability. This capacity increases the consciousness of our diachronic existence as subject to our 
temporal coordinates (past - present - future), and allows us to project the mental image of our existence.

\subsection{Analogy and Self-synthesis}

I have previously emphasised biological mechanisms determining the formation of the secondary consciousness. Let us now underline the linguistic process by which the Self synthesises itself. I will argue that the synthesis of ourselves bears an analogans relationship to an adult-infant interaction.

In chapter 1.6 of this dissertation, I underlined the Iinguistic inclusionexclusion mechanism in the adult-infant relationship (Olivetti 1992). By this cognitive process, the subject identifies its individual specific and species specific identity. During development, infants learn both linguistic mechanisms. The analogy between an infant and its interlocutor is the base for future reflexive self-reference. The first Self-synthesis mechanism is a process of exclusion. I say 'I am X, because I am not you $-\mathrm{Y}$ '. The second mechanism is a process of consciousness of my existence (Brunner 1978): 'I am not You' $\Rightarrow$ 'I am a Self'. This inversion of tendency is taken when I recognise the analogy between your presence as an 'experienced object' (external presence) and my existence as 'agent of perception' (an internal presence). The consciousness of my capacity to perceive your external being compared to my 'inner being' defines my presence as 'objectsubject'. I am aware that a boundary exists between my 'external-internal' presence, and this boundary delimits my physical existence. Your similarity induces me to think of you as an agent-of-perception like me.

When this process is concluded, I can use the word 'We' including in my presence the presence of my interlocutor. I do so because I recognise in my interlocutor an existence that is analogous to mine. Extensive application of this analogan's process induces me to absorb in the word 'We' other analogous entities - 'human beings" (Anderson 1984:35-49, 1991, Bertenthal 1978:44-50, Butterworth 1992 2:103-111, Gopnik 1993). The linguistic reflexive self-reference is based on an analogy mechanism and respects the 'relation of similarity' conditions: I recognise in you an entity similar to me, and I imagine that my features are similar to yours. This is an inductive inference mechanism. This process aims at creating the linguistic reflexive self-reference of my existence as a specific entity. 
In the preceding chapters we saw that Self-synthesis is an intentional action, based on a synthesis agent, embodied in some 'physical production of sounds or marks'. Nozick defined this mechanism as follows:

\footnotetext{
"To reler to anything is to perform an action, with a eertain intention. The token ' $\mathrm{T}$ ' refers to the entity (capable of self-referring) which produce the token; the token 'this very reflexive self-referring act' refers to that action of producing token. Thus, within the reflexive self-referring actions are constituents of an action, intention, causal production." (Nozick 1981:88)
}

The Self synthesises itself around the reflexive indexical emission ' $\mathrm{l}$ '. The word 'I' contains the space delineated by all the self-referring processes: linguistic, intentional and biological mechanisms. However, the milestone of our self-referring properties is the cognitive-linguistic mechanisms of inclusion-exclusion. Without the mental image of our existence as a specific entity the secondary consciousness' mechanisms cannot develop. This mechanism underpins Self-synthesis. In fact, the difference between primary and secondary consciousness is this semantic capacity to create a mental symbol of our existence as individuals. This self-referring property is based on biological features of our psychology. The self-synthesis is obtained when we learn the token 'I', and we cement it in our memory. According to this, reflexive self-synthesis is a process internal to subjects (a reference from their inside), and the Self-synthesis linguistic process refers to our social nature. Even if we focus solely on the autonomy of selfreferential action (the self refers to itself in its self-reflexive action-result), our links with the other human beings creates the tools for our selfreferential processes.

The variability of Sclf-synthesis is explained by the continuous change of our weighting systems in conjunction with the causal relations of our psychological traits. This causality does not represent a unique weightings system and varies in its self referring results. However, the mechanism of coherence in our Self-synthesis is maintained because we continue the 'logic' of this process. In this sense, the heart of our psychological existence moves from the subject's description of mental traits to the subject's self referring process. This qualitatively variable mechanism continues to progressively unify the succession of our Selves in a unitary dimension. This is explained by the organic unity model where a heterogeneous psychological diversity is consolidated into a unity that varies over time. 
The Self synthesises itself and memorises the result of this process. This mechanism allows the consciousness of our persistence over time. When I know that 'Maurizio' is me, this consciousness accompanies my existence. Even if the Self-synthesis is a dynamic process which changes over time depending on my weightings parameters, the consciousness of their being inherent to the entity I am persist. This means that the variability of my weightings system does not obstruct the continuity of my Self over time ${ }^{6}$. My reflexive self-reference synthesises my Self at a certain time and compares this synthesised entity with the memorised past entities that were 'me'. Thus, we can talk about r-related selves that change by maintaining a continuity in themselves. This property relates either to the bodily identity or to the psychological identity. We biologically vary our own constituents in the same way we psychologically change.

Our bodily identity is joined to our past bodily identity because the structural-qualitative nature of the new constituents is strictly determined by the (substituted) old biological sub-elements. In the same way our psychology is based on a fluid r-relationship linking our mental events to each other and to the global entity of 'our self over time'. The Self's reflexive self-reference unifies the subject's experiences in order to allow the Self-unification as a complex organic. In parallel, the organic unity 'the Self' respects the conditions of 'causal correlatedness' and 'maximum similarity'. Since confrontation with our past Selves is a mechanism that recognises our own existence as the (closest) continuer of another entity at a certain (past) time. The Self-synthesis' modalities (our self-referring actions, their confrontation with our past, and so on) infer a causal r-relation within the Selves. The consciousness of our temporal persistence, a singular specific entity, refers to the existence of a Self that continues over time despite its variations.

\subsection{Biological Features of the Self-Synthesis}

In the first chapter of this dissertation, I have posited the organic unity model. This theory considers organisms as a cohesive dimension of heterogeneous constituents, forming a unitary locus of structurally integrated complexity sharing a space time system. The plastic variability of organisms' biological constituents induced me to stress the basic value of the r-relation clustering the heterogeneous constitutive variability. It's qualitative features constitute it as a unitary entity that coherently varies via remaining similar to itself. 
An organism's biological individuality, in this sense, has been defined as a 'process of coherence' based on an r-relation within the organism's constituents. This 'logic of unification' connotes the biological individuality as a variable organic unity. We can say that the biological existence is a mechanism of organism-synthesis in itself. This is explained by its structural-functional causal correlatedness and fitness value, that relates the living being to its environment ${ }^{7}$. Although the Self synthesises itself internally, it bases this process on its social nature (or its perceptive stimulus), in the same way the organism exists as an integrated and correlated heterogeneity. This fluidity refers to its socio-environmental conditions and has a fitness value.

The unification of an organism is not only its interconnection within related constituents, but a mechanism of continuous replies (and memorisation) to environmental and social stimulus. The biological unification is a mechanism that adapts itself to an organism's life conditions, which changes through time, and then reorganises its internal qualitative-structural organisation. This process is regulated by integrated biological systems that have an identical function, to unify. The heterogeneous and variable constituents of an individual remain as a unitary entity despite its variability. One can think of a biological individuality as a 'process of coherence' based on a 'unitary' logic connoting the individual in a diachronic existence.

These biological properties become evident when we view the biological features of the central nervous system. In fact, the synergetic nature of neural phenomena (either sensorimotor replies or neural transmissions) stresses the radical cohesiveness of an immensely complex structure of data elaboration-transmission. The formation of neural maps, feed back mechanisms, mechanisms of categorisations, all these neural mechanisms are based on a process of unification and interrelation.

This involves that the biological property of mind and body respect the organic unity model's features. Since we have stated that mental events are strictly grounded in (but not completely explained by) biology, then the biological and psychological identity of an organism is a unitary dimension and is both structurally and analogously organised to unify the global organic unity complexity.

\subsection{Analogy and Self-Unification}


I began this chapter by anticipating the thesis that organisms synthesise themselves at transversal and longitudinal times via a bipolar analogous mechanism: Self-synthesis and biological existence. We can summarise the obtained results as follow: 


\begin{tabular}{|c|c|c|c|c|}
\hline & $\begin{array}{c}\text { Organism's } \\
\text { Structural } \\
\text { features }\end{array}$ & $\begin{array}{c}\text { Organism's } \\
\text { Qualitative features }\end{array}$ & $\begin{array}{c}\text { Selfs } \\
\text { Structural } \\
\text { features }\end{array}$ & Self's Qualt tative features \\
\hline $\begin{array}{l}\text { Caus } \\
\text { ality }\end{array}$ & $\begin{array}{l}\text { The organism is } \\
\text { a dimension of } \\
\text { integrated } \\
\text { systems } \\
\text { causally } \\
\text { correlated. } \\
\text { Every level of } \\
\text { the integrated } \\
\text { orgatnic } \\
\text { complexity is } \\
\text { causally linked } \\
\text { to the others. }\end{array}$ & $\begin{array}{l}\text { In an 'organism': } \\
\text { each level of } \\
\text { complexity in } \\
\text { compounded into } \\
\text { new entitics at a } \\
\text { higher level of } \\
\text { complexity. The } \\
\text { coexistence of } \\
\text { systems and their } \\
\text { cohesive integration } \\
\text { causes the } \\
\text { emergence' of } \\
\text { characters that } \\
\text { cannot be deduced } \\
\text { by the knowledge of } \\
\text { organism's } \\
\text { constituents. }\end{array}$ & $\begin{array}{l}\text { The Self is a } \\
\text { unitary } \\
\text { dimension } \\
\text { unificd by an R- } \\
\text { relationship } \\
\text { clustering the } \\
\text { subject's r- } \\
\text { related } \\
\text { psychological } \\
\text { traits. The Self- } \\
\text { synthesis is } \\
\text { based on } \\
\text { reflexive self- } \\
\text { references and } \\
\text { their } \\
\text { confrontation } \\
\text { with the } \\
\text { memorised } \\
\text { synthesised } \\
\text { selves. } \\
\end{array}$ & $\begin{array}{l}\text { The Sclf-synthesis refers to } \\
\text { itself and to the causal } \\
\text { (qualitative) continuity with } \\
\text { the past selves, This } \\
\text { mechanism is based on } \\
\text { variable } \\
\text { dimensions and influences } \\
\text { either self-synthesis at a certain } \\
\text { time or self-continuity. The } \\
\text { coexistence of r-related } \\
\text { psychological traits and their } \\
\text { cohesive integration defines its } \\
\text { unitary nature, and the } \\
\text { impossibility to think of a Self } \\
\text { as a sum of memorised data. }\end{array}$ \\
\hline $\begin{array}{l}\text { Simil } \\
\text { arity }\end{array}$ & $\begin{array}{l}\text { As the } \\
\text { organism's } \\
\text { constituents } \\
\text { have common } \\
\text { features (i.e.: } \\
\text { same DNA) }\end{array}$ & $\begin{array}{l}\text { An organism's } \\
\text { constituents have } \\
\text { similar physiological } \\
\text { mechanisms } \\
\text { producing different } \\
\text { results. }\end{array}$ & $\begin{array}{l}\text { Then, a Selt's } \\
\text { constituents } \\
\text { have a common } \\
\text { basis: they refer } \\
\text { to a unitary } \\
\text { variable entity } \\
\text { that is contained } \\
\text { by the indexical } \\
\text { reflexive } \\
\text { emission 'l'. }\end{array}$ & $\begin{array}{l}\text { Then, a Self's constituents } \\
\text { vary in themselves depending } \\
\text { on the weightings' dimensions } \\
\text { chosen by a subject. However } \\
\text { they have a common basis: the } \\
\text { memory and the 'l". }\end{array}$ \\
\hline $\begin{array}{l}\text { Hom } \\
\text { eosta- } \\
\text { sis }\end{array}$ & $\begin{array}{l}\text { The organism's } \\
\text { life is the result } \\
\text { of a structural } \\
\text { interconnection } \\
\text { within different } \\
\text { biological } \\
\text { syslems. }\end{array}$ & $\begin{array}{l}\text { The organism's life } \\
\text { is based on its } \\
\text { homeostatic } \\
\text { structure and adapts } \\
\text { its inner-variability } \\
\text { to the stability of } \\
\text { environmental } \\
\text { pressures. }\end{array}$ & $\begin{array}{l}\text { The self varies } \\
\text { on its own. The } \\
\text { Self-synthesis is } \\
\text { the result of a } \\
\text { structural } \\
\text { interconnection } \\
\text { within different } \\
\text { r-related } \\
\text { psyclrological } \\
\text { traits. and } \\
\text { adnpts the Self } \\
\text { to its life } \\
\text { experiences. }\end{array}$ & $\begin{array}{l}\text { The Self life adapts its inner- } \\
\text { variability to the variability of } \\
\text { subject's experiences and their } \\
\text { influences in self-synthesis } \\
\text { mechanisms. This process } \\
\text { allows the Self's capacity to } \\
\text { adapt itself to the subject's } \\
\text { experiences and to improve the } \\
\text { rational } \\
\text { potentialities. (volitive) }\end{array}$ \\
\hline
\end{tabular}


reflexive self-reference and the Self's continuity. This means that the unification of a Self and its continuity is contingent on two variable dimensions: the self and reality.

Biological and psychological existence mechanisms have a functional analogy in their results ${ }^{8}$. Both refer their unifying properties 'from the inside', to the historical nature of living beings ('subjects of experience'). According to this, an organism's existence is a coherent process of unification of variability in a self-referring entity that is autonomous in itself and simultaneously context-dependent. The variability of 'context' influences the variability of the organic unity 'identity'.

The organism's identity is an organic unity, a locus of integrated biological and psychological complexity that bases its existence on two analogous mechanisms: Self-synthesis and the biological existence. Both these systems are subordinated to the conditions of 'maximum similarity and causal correlatedness' within organism's (psychological-biological) constituents. The variability of these heterogeneous constituents obliges us to think of an identity as a 'process'. The coherence of this mechanism has a tendency to unify a heterogeneous variability in a unitary dimension.

\section{NOTES}

'We could defend the opposite view that supposes the existence of an intimate analogy between things. In this sense, by using - for example - Platonic philosophy, we could say analogy is not a semantic process but a constitutive relationship between ontological entities. When I state that objects have an eidetic source, the role of analogans agent becomes the recognition of a link between the thing's ontological similarity. The analogy becomes a link between 'similar' ontological entities. However, the concept of analogy does not coincide with the similarity concept. To the contrary, it involves a level of dissimilarity that obliges the observer to search for another kind of relationship linking two objects. We can talk about analogy when a level of dissimilarity exists between things. In my view, analogy is an open space within which the observer recognises the similarities-dissimilarities among things and links them when they have an analogous function.

${ }^{2}$ Clearly, when I said that analogy relied on a referent, I rejected the ontological view that considers reality to be the expression of an eidetic source. In a reductionist approach of reality, we could consider the relationship amongst things as only external links, not internal. We have a hermeneutic approach to reality when we make contingent its interpretation. This approach is based in the heuristic tools that we use to explain objective reality. ${ }^{2}$ Another variation on the theme is that analogy is an external property, and not a constitutive property of existence.

'These philosophers defended a similar thesis: we cannot think of the subject as the boundary of the world. In fact, phenomenological, ontological and linguistic peculiarities of our dirchronic existence as subjects link our subjectivity to the reality's objectivity. We can say 


\section{I.9. The Organic Unity 'Species' and its Unification}

Individuals having primary and secondary consciousness derive these capacities from the development of their central nervous systems. Such nervous systems have been selected for in evolution and thus a link between natural selection and the emergence of the mind has to exist. Why have organisms developed / evolved mental capabilities? In this chapter, I will show that cultural phenomena and social behaviour strongly influence a species' existence. I will defend the thesis that natural selection allowed the affirmation of mental capacities (the constitution of primary and secondary consciousness) because mental capacities increase the subjects' adaptive capacities (fitness value). Why has evolution favoured the emergence of mind?

I approach this problem in a neutral way. I do not claim that rationality is positive. I use the biological rationality concept: the individual's capacity to intentionally perform an act ${ }^{2}$. In my work I use rationality as the individual's capacity to repeat complex sensorimotor acts, to memorise those acts and the presence-continuity of the subject. I will analyse the reasons that favoured the evolutionary emergence and consolidation of topographical neural structures that are increasingly complex, and mental capabilities that grow more sophisticated. I will first analyse the affirmation and consolidation of specific gene-lines favouring particular neural topographies at a population level, and then look at the consequences of this phenomenon under natural selection. This methodological approach involves explaining: (1) the emergence of the mind in different species, and (2) its role in species-specific social and behavioural programs.

\subsection{Evolution and Rationality}

Evolution is a causal mechanism influencing the life forms' continuity over time, and influencing the physical (biological) expressions of species-specific individuals. This obvious assumption denies theories that defend the autonomy of mental states relative to the brain, that is, those theories where mind is viewed as an added ontological entity or element. If the disembodied mind did not have a direct link with the physical world (Popper 1987:139-153), we ought to conclude that it is not linked to evolutionary pressures (or influences). But the mind is the expression of a 
physical biological system (the brain) resulting from natural evolution. Thus, we do not have any reason to consider the mind as an a-historical entity. Why is rationality useful in an evolutionary perspective?

Evolution is a natural process that increases and favours the species' fitness value. This mechanism is oriented to facilitate reproductive capacities. If we realise that evolution has a logic, the central problem in an analysis of the evolutionary reasons for the emergence of the mind will be the finality of this mechanism and not the reasons for the emergence of a specific neural structure.

The affirmation of specific phenetic features at a population level, refers to genetic mutations that have been 'selected' and successively consolidated in species specific gene pools (population genetics). The links amongst the members of a species favour the affirmation of characters which in media would be equally possessed by species-specific organisms (Sober 1984: chap. I). This means that, even if natural selection is linked to single organisms in their phenetic individualities, the affirmation of the speciesspecific selected genomes causes the emergence of specific phenetic features at the population level.

We can clarify the relationship between organisms and species by introducing the deme concept. The deme is the 'population'. It is the intermediary link between single organisms and their species. At the deme level, evolution causes individual selection and "leads to the maintenance of fitness of the population through stabilising selection" (Mayr 1988:458). In parallel, the affirmation of a species' selected characters is submitted to the 'adaptation' and 'diversification' condition. That is, evolutionary mechanisms rely on genetic consolidations of certain genetic recurrences at the population level. However, they are not completely explained by changes in gene frequency at the population level. The genes are the vector and the container of the biological transformations, but their selection refers to the phenotypic selection at the deme level ${ }^{3}$.

If evolution is a selection mechanism of probabilistic adaptive-reproductive functionality, why has rationality been selected? We should respond to this question through the logic found in natural evolution: the role that rationality plays in an organism's adaptive processes.

When we think of organisms as the result of natural selection we have to think of the continuity of this phenomenon. For example, if we say that the cerebral topography of hunan beings is the result of the affirmation of a particular genetic recurrence, we have to think of this phenomenon as something resulting from the past and continuing through time. We cannot 
think of organisms as abstract entities, because they are historic entities. We can discuss the dynamics and modalities of genetic evolution (a highly controversial topic in biology), but we cannot deny the dual property of living beings as the result of inherited data, which will be transformed and transmitted to descendants. I defend the thesis that:

The role of the central nervous system is to reinforce the phenetic adaptive potentiality with the individual capacity to intentionally choose behaviours oriented to favour its survival. Rational capacities can accelerate the individual responses depending on the variability of environmental conditions.

\subsection{The Role of Rationality in Evolutionary Dynamics}

To analyse the biological meaning of the mind we must first investigate the behaviour of animals and subsequently look at the rational capacities of individuals possessing secondary consciousness. The biologist Mayr (1976:23) wrote that in the natural world two kinds of behavioural programs existed: Closed Behavioural Programs and Open Behavioural Programs. The first program organises animal behaviours and contemplates certain replics to environmental conditions without alternative possibilities. The second allows a 'space of choice' in the decisions (or alternative solutions) of an organism. Natural evolution favoured the consolidation of the second program. The reasons for this assertion are numerous. Remembering negative behaviours (for example, an act resulting in pain) allows animals to choose alternative solutions in their own lives. Behaviours causing the death of an organism would be rejected, to favour the animal's survival. At the same time, the memorisation of complex sensorimotor reactions to external conditions (for example, how to jump a dangerous obstacle), increases the organism's capacity to quickly repeat complex sensorimotor actions in dangerous circumstances. At another level, the memorisation of dangerous behaviours allows an organism to predict dangerous situations and to abstain from repeating dangerous behaviour.

This capacity increases the organism's fitness value. It is consistent with natural evolutionary laws. The consolidation of open behavioural programs (and the affirmation of complex neural topographies) is favoured by natural selection because it offers organisms the possibility of adapting themselves. Their behaviours are adapting to the variability of natural world circumstances. This mechanism is based on memorisation processes 
(Edelman 1989, 1992). The fitness of behavioural memorisation refers to an organism's subjective capacity to attribute a value to its memorised experiences (mental categorisation and de-categorisation processes). This property belongs to organisms having a primary or secondary consciousness. At the same time, the capacity to substitute instinctive behaviours with intentional choices within different behavioural possibilities increases the plasticity of the organism's adaptive capacity. Those individuals that can intentionally choose will adapt their innerbehavioural-variability to the external environmental variability. The organism's volitive capacity links the organism's future (possible) behavioural choice to its past experiences and reinforces this process by predictive analyses ${ }^{4}$.

The possibility of predictive capacity can be tackled at different levels of complexity. The simplest level relates to organisms with a primary consciousness. In these cases, memories of dangerous situations in the past cause animals to avoid particularly dangerous circumstances of a similar type in the future. This kind of prediction is not really a choice within different behavioural possibilities, but a recognition and remembering of dangerous situations / behaviours. Individuals with a primary consciousness use the open behavioural programs in a limited sense. They can only measure the present choices via the memory of their previous experiences. Their choices are influenced by their past because primary-consciousness animals cannot create mental images of their own identity. They are unable to base their predictive and volatile choices on an awareness of future continuity. The very possibility of perceiving this second predictive level involves individual self-awareness and the possibility of having mental symbols.

Secondary consciousness is a complex phenomenon grounded in an individual's self-awareness capacity to be an identity that continues over time. When this consciousness is memorised, individuals with secondary consciousness can project the mental image of their existence into the past or future. The predictive action will be completely realised because these subjects can 'imagine' themselves and their actions in the future. ${ }^{5}$ They create mental symbols of their own identity. Self-awareness, it seems, is a fundamental property of rational choice. It interacts with the individual (biological psychological) cohesiveness in the heterogeneous dimension of 'the organism'. The subjective self-awareness capacity condenses the individual's mental dimension, and gives a subject consciousness of its 
existence-continuity. According to this, rationality interacts with the unification processes of the Self.

Rationality can be defined as the individual capacity to choose among various potential alternatives and to predict their consequences. ${ }^{6}$ The relationship between evolution and rationality becomes evident when we think about the mind/ brain as the result of a phylogenetic process. Since rational activity is the expression of the brain's neural topography, (which is the result of natural selection), the consolidation of neural structures and their functionality has evolutionary roots. This finality is to increase the organism's fitness value by amplifying the individual's creative adaptability to the environmental variability. That rationality is therefore a biological adaptation, which has a function (Nozick 1993:chap. IV): To improve the organism's fitness value by the consciousness of its presence-in-the-world, and its capacity to adapt its inner variability (the organism is a homeostatic structure) to the environmental variability. This capacity has an evolutionary purpose because it increases the subjective possibility of adapting itself to a variety of environmental changes ${ }^{7}$. This mechanism influences the degree of organic unity, or the 'organism and its species'. This means those nonmerely biological factors, such as culture, language, and social relationships, (Fogel 1993) become unifying factors that make the Selfsynthesis process potent (Kenneth 1968).

The Self is a homeostatic structure because it unifies itself by continuous adaptation to external life-conditions. It maintains a plastic variability in itself that is influenced by the external environmental variability and constitutes its reply.

\subsection{Culture as a Fitness Vector}

I have said that rationality is a biological phenomenon. According to this view, we have to clarify (1) why the concept of rationality is similar to that of homeostasis, and (2) how cultural and social phenomena interact with an individual's existence. Evolution favours an organism's capacity to maintain its structural stability in regard to environmental variability. We can labell this plastic or homeostatic capacity. In the first chapter of this dissertation I used the homeostasis-concept to stress the plasticity of biological structures. The organic systems' homeostatic capacity assures the individual's (biological) stability in the face of environmental changes. Clearly, this biological property has been favoured by evolution, because it 
enhances adaptive capacities at the individual-specific and the speciesspecific level. We can consider this mechanism a basic process of natural evolution (Nozick 1993). If we consider a species to be a composite unity, we could think of its continuity as a homeostatic process. This process would be one in which the species-specific constituents adapt their biological structures to the environmental conditions of their habitat.

Lelas" survival thesis stated that "the better correspondence between cognitive structure and the environment, the better chance" (Lelas 1989). Numerous evolutionary epistemologists (Callebaut 1995) have criticised this idea. However, when we realise that organisms are "cognitive systems" in dialogue with the environment that result from natural selection, the fitness value of rationality becomes clearer. Why has evolution favoured the emergence of rationality if rational capacities did not favour individuals' fitness-capacities? It is a fact that organisms that have the highest rational capacities (human beings) have a predominant position in nature. Their presence is strong enough to subjugate ecosystems to their needs and expectations. Surely this predominance refers to cultural transmission? ${ }^{8}$

This mechanism of structural variability connects with another mechanism of behavioural variability. The rationale has the function of linking the homeostatic structure of the 'organism' to the variability of environmental conditions.

The possibility of choosing different behavioural programs reinforces a subject's adaptive plasticity toward changes in the environment. It is a mechanism that preserves an individual's continuity through time (Cosmides 1989:187-276). This does not mean that all behaviours result from rational choices. The subjective predictive-intentional capacity is influenced by emotional pressures (feelings, beliefs, memories) which personalise an individual's choice. The predictive and self-awareness capacities allow a subject to be a free agent of rationality. This position allows subjects to evaluate the risks and consequences of its choices or simply pursue an action despite its consequences (e.g.: suicide).

Individual freedom (free will) exemplifies the plastic 'organismenvironment' relationship in superior mammals. The rational activity helps the organism's adaptability in a continuously mutable reality. Rationality is a functional tool that can adapt the organism's homeostatic structure to the variability of its life-circumstances. Therefore, reflexive self-reference has a biological function. Self-awareness reinforces fitness-capacities, and increases the organic unity's degree of unification. Given this, an organism's existence refers to the coexistence of two dimensions: biological 
and psychological identity. Both of these elements are intrinsically related and have an analogous function: to unify the individual on its own and to ensure its adaptability to environmental factors.

Natural selection favoured emergence of homeostatic-structures (organic systems), and in the same way favoured the emergence of mind (remember, the mind is in line with evolutionary logic). In short, evolution led to a process that favours the emergence of complex, variable homeostatic systems.

The functionality of this dual system remains the same: to preserve the homeostatic structure of the 'organism' and to favour its diffusion through time. This last mechanism is linked to reproduction, biological variability, and the formation of global units (species). Rationality therefore has a species-specific function, fitness value.

\subsection{Rationality as a Species Unifying Property}

If the homeostatic role of rationality in evolutionary processes is clear, then the role of cultural phenomena in a species' existencemechanism is not. In the third chapter of this dissertation, I explored the struggle to explain the species concept via the sum of its members (I used Hume's individual-nation concept). Using the example of the Homo sapiens species showed how non biological species-specific features concur in existence. These elements reinforce the individual consciousness that applies to a specific species, and aims at compacting the species-identity into a unitary dimension.

This mechanism has evolutionary aims, and identifies a difference in intraspecies' complexity.

Some biological theories have defended the active role of culture and rationality in species' diachronic existence. In 1976, Dawkins proposed the existence of units of cultural transmission: memes (Dawkins 1976:206). These units are subordinate to evolutionary laws, and evolve throughout time (via meme pool) by 'leaping from brain to brain'. Memes are capable of instructing behaviours. Ideas, books, and inventions are all memes (Campbell 1979), whose existence depends on the continued existence of physical vehicles such as brains and objects.

"Examples of memes are tunes, ideas, catch-phrases, clothes fashions, ways of making pots or of building arches. Just as genes propagate themselves in the gene pool leaping from body to body via sperm or egg, so memes propagate themselves in the meme pool by leaping from brain to brain, via a process which, in the broad sense, can be called 
imitation. If a scientist hears, or reads about, a good idea, s/he passes it on to his colleagues and student. ... If the meme catches on, it can be said to propagate itself, spreading from brain to brain." (Dawkins 1976:206)

Memes are thus units of cultural exchange. Independent replicators are similar to viruses in that they infect an individual brain and continue over time by evolving and replicating. Memes are instructions for producing behaviour, stored in a brain and passed on to other brains by imitation' (Blackmore 1996). However, memes, like genes, replicate and evolve by affirming their own 'positive' traits. The memes' evolution will not necessarily coincide with an organisms' fitness. ${ }^{10}$ A contrary possibility is the promotion of specific memetic traits that could result in disadvantages for adaptation.

Even if Dawkins' view stressed the active role of culture within natural evolution $^{11}$, I do not agree with the meme-centric perspective. This is because meme-centric theory attributes such a high value to memes' activities that it disembodies them. The active role of cultural evolution within natural evolution would be considered absolute by the creation of cultural units that have no links with natural selection. For example, Dawkins emphasises the autonomy of memes from the organic systems' cvolution. Memes can also evolve in a way that promotes advantageous traits (to the memes) that are disadvantageous for the organisms' survival (e.g. suicide). Dawkins emphasises that in particular circumstances (like suicide) in which the meme's vehicles are damaged by the promoted meme's trait, tend to result in that meme disappearing from the meme pool (Dawkins 1982:110-111). If disadvantageous traits for meme's vehicles are not promoted, why 'meme-evolution' should diverge from 'organismevolution'? Since evolutionary processes have adaptive finalities, why don't these principles have this feature? When we say memes tend to evolve in themselves and for themselves, we hypothesise their non-adaptive value. According to this, memes are favoured in natural evolution because they amplify the organism's mental capacities. However, since the survival of organisms is reinforced by their volitive-predictive and intentional capacities, and these capacities reinforce the individuals' fitness-capacity, why ought we conclude memes and genotype-phenotype to be 'notnecessarily' oriented to fitness? If memes instrumentally allow mental phenomena, and these phenomena help organisms' fitness-capacities, where is the tension between 'the biological imperative of our genes, ... and the cultural imperative of our memes'? If cultural units have this active role in 
natural evolution, why do we have to consider them autonomous units? Why not conceive of memes as active factors that co-participate in evolutionary mechanisms? Why cannot we think of memes as functionalagents within the fitness (evolutionary) processes? All these objections impel me to think of cultural traits as individual specific and speciesspecific active. They are functional clustering factors, oriented to improve the individuals' fitness capacity through the consciousness of their individual existence as autonomous entities that adapt to environments via their mental capacities. In this sense, cultural traits enrich individualspecific and species-specific capacities for synthesising their Self. It becomes a creative and plastic dimension that can intentionally choose 'good' behavioural choices resulting in functional fitness. Culture is a mental tool with a fitness value that organisms have to creatively adapt to their environment.

A similar conclusion is defended by biologists who advocate Evolutionary Naturalistic Realism (Campbell 1995, Lelas 1989, Callebaut 1995). They defend the thesis that organisms, as cognitive systems, have "accumulated more and more information about their environment and construct better and better representational structures to correspond with the structure of the environment in which they live" (Lelas 1989, quoted in Callebaut 1995:30). Campbell's view saw a cumulative mechanism of inductive achievements or vicarious selectors (Campbell 1995). These are selected to positively relate organisms to their environments. Campbell synthesised the vicarious selectors' theory as follow:

1. A blind-variation-and-sclective-retention process is fundamental to all inductive achievements, to all genuine increase in knowledge, to all increase in fit of system environment. 2. The many processes which shortcut a more full-blind-variation-andselective-retention process are in themselves inductive achicvements, containing wisdom about the environment achieved originally by blind and selective-retention. 3. In addition, such shortcut processes contain in their own operation a blind-variation-and-selectiveretention process at some level, substituting for overt locomotor exploration or the lifeand-dcath winnowing of organic evolution. (Campbell 1987:91)

Campbell pointed out the existence of numerous vicarious selectors coparticipating in natural evolution, such as vicarious locomotor devices, habit, vision, instinct, thought, language, and cultural cumulation (Campbell 1987:54-73). These selectors increase the adaptive interaction organismenvironment through blind and selective-retention processes. According to these, evolutionary mechanisms involve a synergetic and composite process 
by which evolutionary adaptation covers biological (genotypic and phenotypic) features of organisms and 'cultural' vicarious knowledge processes, including vision, thought, imitation, linguistic instruction and science (ibid. p.89). Both biological and cultural evolution are equally involved in evolutionary processes and are finalised towards adaptation. This idea connects all the ENR advocates as has been clearly explained by Sir Karl Popper. The English philosopher sketched the reasons for the emergence of mind as follows:

1. The beginnings of mind and consciousness should be favoured by natural selection simply because they mean the substitution of imagined or symbolic or vicarious behaviour for real trials that if erroneous, may have fatal consequences ... 2. The open program allows for possibilities to be played through tentatively ... in order that selection can be made from among possibilities. 3 . In connection with open choices, a fecling may evolve of preference for one possibility rather that another. 4. The evolution of language ... allows us to dissociate ourselves from own hypotheses, and to look upon them critically. (Popper $1987: 152)$

This points to a clear link between natural evolution and cultural-mental processes. The evolution of living beings and their mental capacities is a synergetic, not antagonistic process. (Dawkins: 1976) Natural evolution has favoured the emergence of mind in order to increase organisms' creative and autonomous approach (prima persona) to environmental changes. This mechanism allows the supremacy of those individuals that developed the best rationale (volitive, predictive) capacities. This process results from natural selection and has a coherent evolutionary logic: to favour the best 'adapted' individuals. This conclusion, however, does not involve the "mind versus body" relation (Dawkins 1976, Dennett 1995). It simply differentiates the coherence mechanism in natural evolution. Living beings that have a greater degree of complexity in their organic unity have higher adaptive capacities. Individuals that have a higher degree of complexity in their neural traits have a greater fitness capacity, because they can easily adapt themselves to mutable environmental conditions. These organisms have a predominant place in the natural hierarchy.

Thinking of the human species from a 'non-biological' perspective, we have the instinctive consciousness that is akin to a global dimension, "the human race'. The nature of this instinctive consciousness cannot be explained via the surn of the 6 billion beings that live in the world. Why do we have this intuition? If species-awareness did not exist, we would be oblivious of our species-specific identity. Nevertheless, we know that we belong to a composite dimension, our species. 
We have seen (chapter I.6) that formation of self-awareness refers to social relationships with other members of a species. Issues from evolutionary psychology, in fact, suggest that natural selection has favoured "an ability to think about social situations and relations" (Kitcher, 1993:300-310, quoted in Callebaut 1995:32). The Self-synthetic processes confirm this socialdependency of individuals in their reflexive self-referential process. The individual specific self-awareness implies a species-specific self-awareness: the individual learns to be part of a composite entity (society, population, species), which is dialectically autonomous in itself and subject-dependent. Linguistic mechanisms (We-l) show the complexity of this process (Gopnik 1993). This is a conditional mechanism, since without social relationships, the self-synthesis process does not even begin. This is because secondaryconsciousness processes are deprived of the subjective capacity to be aware of individual existence (Edelman 1992). This suggests that subjective selfawareness induce the subjective awareness to identify with a composite identity - 'humanness'. Cultural phenomena reinforce this process (Dennett 1991, 1995, 1996, Donald 1993). We learn that a species has a history, then, that this history belongs to us. We have the consciousness to belong to a global entity that is separated from our individual presence, and yet linked to it.

Let us continue thinking about our own society ${ }^{12}$. We think of society in the third person singular, but we also know that it is composed of many individuals. We are conscious of our belonging to this composite dimension, and at the same time, we think of society as something separate from our presence. This mechanism refers to the individual consciousness that the society is an identity's macro-level, composed by us, but not exhausted in us. We inherit historical bio-data (our genetic identity), and we also inherit mental-cultural requirements. This assures the consciousness of our presence in reality as a member of a species. This second mechanism is orientated to reinforce our mental self-awareness, and exemplify the evolutionary role of rationality and culture in species (individual) existence mechanisms. Since natural evolution is a mechanism aimed at improving the fitness value in animal species, culture and social behaviour, an evolutionary perspective tends to reinforce the individual self-consciousness with a species-specific consciousness. ${ }^{13}$ This acknowledges that: The process of evolution increases the fitness value in organisms via rationality because the rational capacity of species-specific members is reinforced by a 'container' of information that amplifies the rational potentialities in 
subjects (we learn by culture), and is extended to species identity. Rationality has a fitness value

Subjects synthesise themselves (their Selves) through tightly knit social relationships to maintain the society/individual union, and through culture. Culture is a container of information aimed at adaptive processes, where subjects amplify the consciousness of their rational potentialities. This system is conditioned by social behaviours, because society interacts with the cultural and behavioural selection of its members (Nozick 1993). Thus, rationality connotes species, and species influence the rational-behavioural features of species-specific members. A mutual causal relationship between the species' micro-and macro level exists. Species specific individuals have rational capacities for being reactive and autonomous toward their habitat (adaptive capacity). Species favoured the emergence of rational capacity to increase their evolutionary affirmation, and to reinforce their adaptive biological capacity with an evolving mental one.

\subsection{Culture and the Emergence of a Species-Specific Self- Consciousness}

Let us approach another example of species-specific selfconsciousness formation: the historical meaning of a species. When we explore our species-specific history, we enrich our self-consciousness. It becomes an identity that dialectically continues on its own through time, and belongs to the macro-entity (human society) resulting from a past and continuing into the future. For instance, historical knowledge of our nation shows us that we have inherited cultural features and their psychological expressions, and that such influences can connote our individual-specific behaviour. The knowledge of these cultural roots reinforces the consciousness of my belonging to a global entity - 'the Italian people' - and of my own 'individuality'. In saying this, I am not defending a simplistic generalisation of behavioural features, but rather suggesting that we receive a set of cultural inputs (conscious or unconscious social behavioural programs) which are selected by society and used in social maintenance. This does not mean that our individuality is absorbed by society, it simply means that we have a cultural background, a 'mentality' (or value system). This 'received' information can be critically analysed by us and consequently influences our behaviours. According to this, the consciousness of our belonging to a species-specific entity that has a historical and behavioural feature reinforces our existence as individual 
self-conscious' subjects. Natural evolution uses culture to transmit selected behavioural programs, contained in culture, and expressed in individuals' unconscious approaches to reality. ${ }^{14}$ The cultural influence aims at compacting the individuals of a 'population', and linking the members of a population via maximum similarity and causal correlatedness measures. Rationality has the role to synchronically favour the individual specific identity (the Self-synthesis). It unifies species-specific identity through the individuals' consciousness to be part of an entity that has specific features on its own over an extended period of time (Donald 1993, Dennett 1996).

In this sense, cultural and social phenomena aim at unifying the global organic 'species' (and society) on its own. This mechanism refers to rationality because rational activity allows for species-specific awareness, and has adaptive aims (Popper 1987, Nozick 1992). The organic unity (species) exists in itself, and has 'non-biological' clustering factors that ensure its continuity over time and link it internally to its constituents (single individuals).

In this perspective, the role of rationality at the species-specific macro-level is to assure the individual of the possibility if using rational tools for inventing new adaptive solutions. Culture guarantees the transmission of different behavioural programs (having a fitness value) which are selected by evolutionary (cultural) mechanisms. We can use these tools in our individual approaches to reality. This mechanism is highly functional in finding new behaviours that produce adaptive ends. Culture increases this rational potentiality and interacts with the species-specific unification process. Different species have different mechanisms of behavioural program's transmissions.

Another important link between rationality and evolution refers to the speed of biological change. The qualitative-structural biological transformations are slow. Even if phenetic evolution is faster than a genotypic one, when we compare cultural and biological evolution we observe an incredible discrepancy in their rate of change. This phenomenon can be explained by underlining the active role of the mind in evolution. Since the external variability of environmental conditions (climatic, economic, and nutritive resources) changes quickly, natural evolution uses rationality to maintain a balance between environmental changes and the survival of a species. A species with a greater capacity for rationality can adapt quickly to environmental variables. We could hypothesise that rationality allowed the predominance of the human race in the biosphere because we have the greatest capacity for rationality in our ecosystem. This 
predominance of human rational capacity rests on the use of linguistic systems for the transmission of past experiences (behaviours), and the use of mental symbols in their predictions. Human beings use their past to create habitats in which survival is safeguarded. This capacity extends to the creation of abstract (formal) explanatory tools and technological instruments able to explore the natural world despite the limitations of human senses. The predictive capacity is oriented towards safeguarding the organisms' survival. Rationality is a powerful tool in environmental factors. The fact that we are not only adapting ourselves to our environment, but also the environment to ourselves, is a 'privilege' of rationality. The emergence of the mind has evolutionary ends, and is substantially oriented towards increasing the individual-specific/species-specific fitness value (Di Francesco 1998).

To summarise, I have defended a view that emphasises the role of rationality in evolutionary dynamics. Rationality increases the fitness value of organisms towards the variability of the natural world (Popper 1987, Nozick 1992). Cultural and mental mechanisms cause the individualspecific self-awareness to be-in-the-world. This property has evolutionary purposes because it improves fitness mechanisms. In this process, culture has an active role because it transmits the species-specific information and amplifies the individual specific (and the species specific) fitness value. In this view, the emergence of rationality allowed the affirmation of organisms with secondary consciousness because they have a superior fitness potentiality. This mechanism aims at accelerating the organisms' adaptive processes towards environmental changes.

\section{NOTES}

\footnotetext{
'Questioning the rationality problem in an evolutionary perspective does not mean that I aim to defend human supremacy towards other animal species nor that I consider rationality as uniquely human. It is obvious that we can base an inter-species comparative analysis on other species-specific features than rationality. For example, adaptive capacity could be taken as the measure, by which a mosquito would win over a human being. In Nature, however, the prevalence of rational living beings is evident so we have to consider, at least, the reasons for emergence of this phenomenon in an evolutionary perspective. Evolution is a natural pressure increasing the functionality of biological forms in their adaptive and reproductive capacities. At the same time, this process has a probabilistic nature, since natural selection favoured organisms having higher reproductive potential.
} 
${ }^{2} \mathrm{My}$ rationality concept is biological: rationality as the subject's capacity to perform or repeat an action. In this sense rationality is connected to neural topography. It is an individual capacity that increases its predictive and intentional choices. Clearly, the increase of organisms' neural complexity will be extended to the complexity of their mental events. Individuals possessing secondary consciousness have a higher rational capacity than those possessing only primary consciousness. They can link their choices to their past experiences, predictions, believes, fantasies, conjectures, refutations, and so on. $A$ schematic analysis of the rationality concept is found in Bartley 1987: 205-214.

${ }^{3}$ In the chapter 1.3 of this dissertation we have seen that a species is a composite and hierarchical entity: individuals $\Rightarrow$ populations $\Rightarrow$ species. We also have seen that species' identity exists in itself even if species is a biodiversity. The deme-level transmits to individuals a whole of selected features (resulting from the natural selection) by which they can improve their fitness-value.

${ }^{4}$ The fitness value of mind is completely different from that of other biological systems. For example, if we think of the immune system, we see that organism can biologically react to environmental factors (reply to infectious diseases). In this case the organism cannot intentionally increase this inherited system even if its immune system learns how to react to a disease (let us think of the antigen-antibody relationship). To the contrary, the cognitive capacities of individuals possessing secondary consciousness rely on their capacity to learn by past experiences and to increase their own reply- factors.

I am aware that predictions of future consequences rely on induction and analogy mechanisms. Nevertheless, in this dissertation I am limiting my analysis to the evolutionary reasons of the emergence of rationality in naturc. Other problems in the philosophy of mind such as 'rational choices', 'decision theory ', 'rational inductivism' etc., cannot be analysed in this dissertation because they are not in accordance with the economy of this work. However on this topic see: Jeffry 1965, Levi 1986; Lewis 1981:5-30; Campbell \& Sowden 1985 ${ }^{6}$ Philosophical theories used the rationality concept in different ways. $A$ rational choice is taken by a subject when among different possibilities $x, y, z$, to perceive a determinate goal $N$, he chooses the best solution that can guarantec the optimisation of $N$ by the chosen possibility (e.g.) $x$. This means the rational choice needs mechanisms of prediction, cohcrence, free will, utility-maximisation, and so on. It is clear that the emphasis on a particular parameter participating in the complex mechanism "rationality" interacts with the concept-detinition of rationality. If we link it to socicty, for example, we have a direct connection between $\mathrm{N}$ and the social welfare, or a contractualistic (or utilitarianism) ethical connotation. The value of $\mathrm{N}$ in this view becomes a point of reference that influences the meaning of rationality. Wc can use logical tools (decision theory) or social tools (Simon 1983; Schulz 1971), however we pass from the problem of rationality in itself (as a biological plenomenon) to its expression in selfaware subjects living in a society. Since I attempt to clarify the meaning of rationality despite the social-ethical implications of rational choices, in my work I will consider rationality as the capacity to repeat complex sensorimolor acts, to memorise them and to memorise the presencecontinuity of a subject. In this vision, other mental features increasing the complexity of rational activity (memorisation, use of mental symbois, language, self-awareness, ctc.) will be important in defending a specific thesis: rationality is a mental process that has different levels of complexity. The presence of multiple complexity factors increases the complexity-rate of mind. This means we can use rationality as a comparative parameter to define a difference of complexity among different lifeforms. In this view, I will be interested in the subject as an 
agent of rationality. The social-environmental-ethical connotations of $\mathrm{N}$ will not be important since I am investigating the meaning of SI and not the meanings of organisms' actions.

7 "In the process of biological adaptation by variation and selection, living beings, as cognitive systems par excellence, accumulate more and more information about their environment and construct better and better representational structures to correspond with the structure of the environment in which they live" (Lelas 1989:141)

8 "Human beings owe their biological supremacy to the possession of a form of inheritance, quite unlike that of other animals: exogenetic and exomatic heredity. In this form of heredity information is transmitted from one generation to the next througb nongenetic channels ...; in general by the entire apparatus of culture" Medawar 1977:14, quoted in Dennett 1995:342

9 "Examples of memes are tunes, ideas, catch-phrases, clothes fashions, ways of making pots or of building arches. Just as genes propagate themselves in the gene pool by leading from body to body via sperm or eggs, so memes propagate themselves in the meme pool by leaping from brain to brain via a process which, in the broad sense, can be called imitation." Dawkins 1976:206

${ }^{10}$ Biologists and evolutionary epidemiologists have reacted differently to Dawkins" hypothesis. The idea that genes are not the only replicators in biology has been criticised by those scientists that have proposed a direct link between cultural-evolution and genetic evolution (sociobiology). One of the best advocates of meme view is Dennett. In his book (1995) he defended the thesis that the meme is a separate replicator and described its replicating power in terms of the fundamental algorithm of natural selection.

11 The meme-centred perspective and the gene-centred perspective are two parallel principles through which natural evolution occurs. "There is a persisting tension between the biological imperative of our genes, on the one hand and the cultural imperative of our memes on the other" (Dennett 1995:365). This tension does not mechanistically connote organisms' behaviours or mental phenomena. It simply accompanies an individual's biological needs, encoded in its genes, to memes' needs contained in its brain. It is not necessary to explain natural evolution as a mechanistic process oriented to preserve gene needs. On the contrary, it is influenced by cultural phenomena as expressed in socio-behavioural programs, and biological processes such as adaptation and reproduction. Mernes influence natural evolution in order to safeguard their own needs.

${ }_{12}$ The socio-psychological mechanisms involved in the synthesis of the Sell have been decply analysed by sociologists and psychologists. I do not attempt to approach this topic in its complexity. However, you can find significant implications in Babad, Birnbaum, and Kenneth 1983.

${ }^{13}$ The fitness value of rationality is extended to spccics because we have cultural backgrounds allowing us to increase our mental (cultural) and rational capacities.

14 Nevertheless, since natural evolutionary attempts to increase individual-specific's fitness capacities, this cultural heritage reinforces the individual freedom in those subjects who rationally want to use new behavioural programs in their own life. 


\section{Conclusions: Biological Problems.}

In the first section of this dissertation I addressed the identity problem through an interdisciplinary approach. I focused my research on the biological and philosophical problems involved in the concept of individuality. This methodological approach may be criticised by emphasising the limits, or superficiality, of my explanations in regard to the complexity of the involved phenomena. I am aware that most of these criticisms are justified. Because I am not a scientist and my analysis of the concept of identity in natural sciences is not the result of experimental results but one based on mediated data. This dissertation is, however, a reflection on the meaning and the nature of the living beings' individuality as a unitary phenomenon. I thus use different explanatory tools (biology, genetics, psychology, philosophy) to defend a coherent theory that refers to the above mentioned scientific disciplines ${ }^{\prime}$. In order to perceive this goal I have based my philosophical explanation on the biological features of organisms ${ }^{2}$. In this sense, my theoretical approach to identity problems is a consequence of the biological properties of living beings.

Being a philosopher, I have linked these scientific explanations to the philosophical implications they engender. In doing this 1 have not 'forced' the scientific data into a theoretically pre-constituted philosophical view. I have linked the biological features of living beings to philosophical explanations on their existence as specific individuals. In this dissertation, I thus present a methodology of research that refutes the dichotomy 'science versus philosophy', and 'theoresis versus pragma'.

In the first part of this dissertation I have defended a specific thesis: organisms are organic units, they are the locus of integrated biologicalpsychological complexity unified in a heterogeneous and cohesive unitary dimension which varies over time by adapting to environmental variables (where organisms are homeostatic structures). The claim of the organic unity model is:

The organism's identity is an organic unity. A locus of integrated biological psychological complexity that bases its existence on two analogous mechanisms: Self-synthesis and biological existence. The variability of organism's (psychological-biological) heterogeneous 
constituents obliges us to think of an identity as a 'process'. The coherence of this mechanism is its tendency to unify a heterogeneous variability in a unitary dimension. According to this, the central element of identity puzzles becomes the r-relations clustering an organism's components. Also, since an organism is an organic unity, we can think of its identity as a complex process that has variable levels of complexity.

This suggests that the existence of an 'individual' at a specific time, and throughout time, is a coherent process in which separate processes join to ensure the existence-continuity of an individuality on its own. The fundamental property of the organic unity concept is that an organic unity cannot be explained via the sum of its constituents. This condition is respected in biology because natural phenomena portray that the coexistence of biological systems, and their cohesive integration, causes the 'emergence' of characters. This cannot be deduced from knowledge of a specific organism's constituents. The identity becomes a locus of integrated units or diversities: a unitary dimension. The unitary meaning of biological systems allows us to understand their emergent properties.

If we accept this, we cannot say that DNA is the primary determinant of an individual. Nor can we say that 'the mind' plays this role either. Both coparticipate in the life of individuals as physical and phenomenological entities. In this sense, the organic unity concept attests that we cannot approach the individual problem in biology or neurology through mechanistic explanations, because the existence of an individual is a composite process that cannot be explained via a unique causal principle. We thus should think of biological identity as a synergy of biological and psychological processes that have analogous functions. That is to unify the biological-psychological constituents of an individual in a homogenous entity that continues over time.

The organism is an organic unity because it has internal processes of autocohesiveness. The organism can be conceived of as homeostatic system. The homeostatic nature of an organism ensures its adaptive capacity. According to this, those systems that allow the biological integration of living beings have a central role in the existence of individuals. Since these factors are numerous, (from metabolism to the immune system, from neural topography to mental states), it follows that $r$ is a set of factors possessing a communal aim - an aim that joins a qualitative structural biological variability into a homogeneous entity - 'the individual'. The impossibility of 
exhausting the identity on a descriptive level, motived the thinking of identity mechanisms as processes regulated and explained by unifying properties. The identity thus is a unified and variable organic unity unified by an r-relation between and within the organism's constituents.

When we apply the organic unity theory to organisms that have a developed central nervous system, the consciousness problem becomes central. The biological analysis of mental phenomena clearly shows that it is virtually impossible to think of individuals as dualistic entities (Cartesian philosophy). In fact, modern neuroscience proves that there is a strict causal relationship between a subject's neural structures and its neural phenomena. This indicates that mental states result from a physical structure, and that their features are tenaciously connected with the biological characteristics of the subject's brain. In this perspective, the dichotomy body versus mind becomes nonsensical since an individual's psychological identity is the expression of a biological neural complexity.

An organism is a dual entity: it is 'object' (biological) and 'subject' (psychological). When we stress individuals' phenomenological nature we emphasise their reflexive self-reference. The central mechanism of a subject's existence as a specific Self is its capacity to reflexively self-refer. The role of this capacity is to unify the mental states' diversity in a unitary dimension - the Self. The Self thus is an organic unity because it is a variable unity-in-diversity that cannot be exhausted in the sum of a subject's mental events and connotes itself on its own. Based on this premise, we can think of both the Self and the Body as two joined components of an organic unity.

A comparative analysis of Self-synthesis and biological existence mechanisms shows a functional analogy between these systems. This means that Self-synthesis and biological existence are two mechanisms of an organism's unification in its dual nature (mind and body). According to this, the relationship body versus mind loses its juxtaposed nature, while the organic unity concept absorbs into itself both the mental and the bodily properties-peculiarities of a subject. The unitary nature of organic systems, being cohesive heterogeneous dimensions of integrated complexities, respects the heterogeneity of living beings' existence, and denies any form of 'biological-psychological' reductionism. The subject's continuity is based on a specific logic: unifying a variable heterogeneity into a cohesive unitary entity. This process is taken by two analogous mechanisms of unification: the psychological mechanisms (which unify the Self) and the 
biological mechanisms (which unify the body). Both these principles interact and explain the dual nature of organic units.

Two modern philosophers address this bipolar possibility in detail, Nozick and Parfit. In my work, I used Nozick's theory because he aims at defining a subject's conditional property, as well as individuating the metric of a subject's persistence through time. Nozick's closest continuer scheme allows us to solve the individuals' identity persistence dilemma while respecting their dual nature and the variability of individuals' existence.

In the following pages of this dissertation I will apply my theory to a number of case studies in which the individuality of organisms is deeply modified (gene manipulations and cloning). I will explore issues related to subject's continuity via both the organic unity model and the closest continuer schema. The main goal of this effort is to show that the notion of biological individuality can help us in having a better understanding of consequences arising from bioengineering Life.

\section{NOTES}

'On the other hand, the heterogeneity of our topic can be refuted on the general explanatory level of identity by those persons who think the identity problem is based and justified on a specific problematic - or scientific - area. In this sense, a biological identity explanation will be refuted by (for example) a non-reductionistic philosopher and vice versa. However, the synergetic nature of identity problems allows the use of different prospective angles in the identity investigation.

${ }^{2}$ If the scientific super-specialised approach on Identity allowed a positive deep study on identity problems, it looses the unity of the investigated topics: the meaning of the biological and psychological individuality. In fact, if we continuously break the problem into subproblems to investigate, we risk losing the unitary meaning of our analysis. This is clear when we emphasise the organisms' integrated complexity. We can think of organic systems by emphasising their micro-features (from molecules to quanta, or from neural fibres or structures to neurons), but we also have to analyse other levels of complexity (organs, tissues, systems), their mutual interconnection, and the global entity 'the organism'. If we limit our study to a determinate magnitude of complexity, we cannot deny the relationship between the micromacro properties of an organism. Aa theory on biological individuality needs to be coherently applicable to the organism on its own differing levels of complexity. In this sense, I have based my study on problem cases that refer to different rates of biological complexity. Also if 'Maurizio' is formed by billions of cells and organs and neural structures, the is also a determinate Self in itself. With this entity I have measured my dissertation. 


\section{II.1. Conceptual Premises.}

The heuristic growth of natural sciences is impressive. Human society is now in a position to clone mammals, to genetically modify germ cells of human beings and so on. We could have massive benefits arising from the use of these technologies. New territories could be explored in human medicine, new tools could be created for producing safe and nutritious foods, caring for the ageing population, helping sterile couples. Nevertheless, every growth of the human knowledge needs to be implemented by critical studies on the right way to use the available discoveries.

History teaches us that a reflection on the potentialities of new technologies is a pre-requisite for their use. This will help avoiding unexpected consequences (such as changes in the natural evolution of living beings). Or simply to prevent a cascade of events transforming potential benefits in unexpected negative consequences.

A well-known example of this problem is the use of atomic energy for producing terrific destruction-tools instead of helping modern societies with their energy needs. At present similar questions refer to biotechnology (such as the production of GMOs or transgenic crops). They bring scientists, as well as decision-makers, to address questions related to the responsibility of science.

Therefore, I will address in this part of my dissertation a number of case studies where the biological identity of an organism is (a) deeply affected by human factors [genetic modification of human (II.2, Il.3) and non-human (II.4) germ cells] or (b) extremely problematic [sexual reproduction Vs animal (II.5) and human (II.6) reproductive cloning]. Both kinds of case studies are relevant because they concern issues which are related to the persistence of future-born individuals (bio-engineering of germ cells) as well as to the use of bioengineering to manufacture new types of individuals who have an unclear genealogical link with their ancestors (cloning). The above-mentioned case studies have implications not only for the identity of present generations, but also for future generations, reason why we will start our analysis with an exploration of the issue of responsibility of science in the field of biotechnology. 


\section{2. The Responsibility of Science in the Field of Biotechnology}

The responsibility of science was analysed during the 1970's (Edsall 1981:11-14, Stent 1974:41-51; Loren, 1981). The basic difference between the discussion then and current debates is that in the last two decades, science has provoked huge changes in the natural environment (think of the use of pesticides, pollution, atomic energy, and so on). Now, in the recombinant DNA era, the human society can transform the biological matrix of future organisms, their genome. Biologists can create genetically manipulated organisms (GMOs) whose existence is morally problematic because for the first time in the history organisms are manufactured and introduced into the environment by human beings (transgenic animals, GM crops). The 'responsibility of science' then has become a central problem of modern ethics.

One of the main problems arising from the use of biotechnology to genetically manipulated organisms is the safety of such a technology: the Costs-Benefits model $(C / B)$. Decision makers often think that when a technology is safe and offers potential benefits, then it should be considered as ethically legitimate. The responsibility of science, then, is equated with the optimisation of $\mathrm{C} / \mathrm{B}$. In the case of biotechnology, which is the one I am exploring here, we have two possible solutions to the $\mathrm{C} / \mathrm{B}$ problem:

1. Science does not have an ethical responsibility and we should address implications of biotechnology via the Costs/Benefits model (C/B);

2. This responsibility exists and it is not explained by $\mathrm{C} / \mathrm{B}$.

Point (1) denies the notion of scientific responsibility. In this perspective, the positive side of an experiment in biotechnology refers to the optimisation of $\mathrm{C} / \mathrm{B}$. When a technology is effective and safe (the greatest benefits with minimum cost) it is considered 'ethically' legitimate. However, can we consider R/B as the right tool to be used to explore problem implications of biotechnology?

First, it should be noted that $\mathrm{C} / \mathrm{B}$ is an appropriate tool to explore the safety of a technology rather than its ethical legitimacy. Ethical factors do not coincide with the ones of safety (Schroten 1997). These last ones can be relevant in an analysis aimed at quantifying the consequences of a technology (risk assessment study), but they are silent in regard to all those factors that can hardly be quantified, such as the moral principles and values involved (like freedom, dignity or solidarity) In parallel, even if we use C/B 
to explore the complexity of a specific technology, we should be aware that some case studies cannot benefit from such an approach since it is not easy to identify which consequences should be taken into account in a risk assessment study. For example, when we deal with GMOs, should we consider the long-term or the short-term consequences of producing these organisms? The first ones can be foreseen (mortality rates, induced pathologies etc.) but what about the second ones ${ }^{1}$ ?

Usually the problem implications of biotechnology are addressed through two juxtaposed approaches: The impossibility of foreseeing the long-term consequences of modifying germ cells of living beings should induce us (a) not to produce GMOs or (b) to do it regardless of potential risks ${ }^{2}$. Both of these approaches use consequentialist arguments. The first one attributes the unknown consequences with a value strong enough to annul possible benefits, which might result from engineering Life. The second one strictly refers to the expected 'benefits' without taking into consideration the longterm consequences. In the fields of biotechnology, consequential arguments, thus, can be conducive to juxtaposed conclusions: prudential attitudes (Jonas 1979, 1987) as well as liberal attitudes (Harris 1992). Therefore, (a) conclusions based on consequentialist approaches $(\mathrm{C} / \mathrm{B})$ are relative to socio-cultural factors (Salvi 1997), (b) conclusions based on C/B may help us to predict the impact of a technology but cannot be considered as ethical analyses, (c) bioethical analyses should add to $\mathrm{C} / \mathrm{B}$ an inquiry into the ethical factors which are involved.

This last claim refers to point (2) of my previous scheme. If we consider this as a more fitting key for opening the door on discussions of how bio-ethical problems should be addressed, then we should explore the moral factors involved in our problem (values, theories, rights, etc.) and successively link $\mathrm{C} / \mathrm{B}$ model to these moral parameters. In this perspective, ethics would become a central element to take into account in approaching the implications and potential problems of biotechnology.

\subsection{Ethics of Science, Science of Ethics}

It is necessary also to say some words on the role of ethics in science, and the way ethics is used in bioethics. The first end of an ethical analysis is to explain why an action is morally wrong or right. Knowing the type of questions we ask ourselves when we search for what is good or what needs to be done in a certain situation is a preliminary, logical, and 
conceptual point of view from which to express our judgements (Lecaldano 1997, 1999). But it is not possible to use the word 'ethics' as an empty adjective to use when we address problem situations. For example the use of biotechnology to genetically modify organisms becomes an ethical issue when it concerns ethical factors. Bio-diversity, future generations' rights, environmental impact, all these factors are inherent to GMO production. But they can be considered as moral factors only when their value is justified in a moral context. To say that the use of a new technology is "controversial' does not mean that it is ethically problematic (Salvi 1995, 1999). We have social or political controversies based on new technologies -let us think of functional food production-, which could be problematic in a context, which is not the ethical one (for example the argument saying this use of biotechnology could create new job-possibilities). When we overlap different contexts in the word 'ethics' we risk claiming confusing analyses, leading to contradictory results ${ }^{3}$.

The word bioethics intrinsically entails two dimensions that are apparently disjointed: biological, or medical, phenomena and ethical analyses. However, this does not mean that bioethics is a theoretical discipline. In fact, bioethics needs to answer to real and concrete phenomena through a number of mediums. This means that bioethics is intrinsically interdisciplinary, it is a polyphonic science (Salvi 1996), a chorus of different disciplines which when juxtaposed, evidence an undifferentiated whole. One of the voices of this chorus is philosophy.

Indeed, this interdisciplinary approach is not always fulfilled. Scientists talk about the need to define some ethical border, which should be fulfilled in genetic research, but at the same time they do not explain what ethical principles they refer to. In doing so they often defend highly equivocal statements that are hardly acceptable for philosophers. On the other hand, philosophers often address issues related to the use of biotechnology in bio-medical sciences without taking into account the biological implications of the case study they are analysing. Their approach is so conceptual to result unaccepted by scientists. Other experts (lawyers, sociologists etc.) do the same. Then it becomes very difficult to present a view that simultaneously reports these seemingly opposed methodological approaches. However, when we realise that bioethics is a pragmatic science, then we do need to adopt an interdisciplinary approach to the problems to be investigates.

In the fields of biotechnology, this involves that bioethicists should explore: 
- biological features of the object to be investigated;

- analysis of moral implications arising from the use of a specific technology;

- analysis of consequences related to this action;

- analysis of different theories that may be used to solve problems to be explored.

\subsection{The Organic Unity Theory as a model to use in bioethics.}

Thus far, I have emphasised the relationship between theoretical and practical approaches in cases surrounding problematic questions of the concept of individuality. In this last section of my dissertation, I will use the organic theory as a reference in practical cases of identity transformation. In particular, I will emphasise bioethical dilemmas that imply direct consequences for organisms, which are burdened by them. This involves an analysis of both human and animal genetics (germline transformations) and cloning. Other bioethical puzzles, such as the allocation of sanitary resources, euthanasia, patient rights, and so on, will be not addressed in this dissertation. This is primarily because a number of issues on these topics already exist in bioethics and secondly because I do think that bioethics should deal with 'new' puzzles, those linked to modern technology. This idea demands an emphasis on biotechnology, since the huge growth of this arena urges human beings to ask themselves; what are the moral implications of a policy based on engineering Life? Now, that it is clear where I stand, let us sketch some more pictures and try to visualise a few of the problems plaguing these new discussions.

1. Biotechnology is an example of the human interaction with Nature.

2. Biotechnology allows for the production of 'new organisms' (for example transgenic animals, or chimeras).

3. Biotechnology is a science that frightens society (cloning is rarely portrayed as positive).

4. Modern ethics does not offer critical or sufficient answers to the problematic implications of biotechnology.

In order to explore these statements 1 will begin by focusing on biological consequences of bioengineering Life. To support this discussion, 1 will follow it up by linking these results to philosophical explanations of genetic 
transformations. In this format, I will present a theory that conforms to both conceptual and methodological approaches.

Analytic philosophy will be the explanatory tool with which I carve and strengthen my positions. I am aware that such a methodological choice can appear odd, because the moral status of biotechnology has been previously depicted and discussed through other mediums. However, I hope that this will, at the very least, display how biotechnological problems can be allocated different value with different logical grammars. The analysis of problems related to the organism's identity produced by experts of analytical philosophy, give us significant means with which to study the consequences for the identity continuation of a subject undergoing a germline gene manipulation. My choice of methodology encourages me to work with the analytic method, breaking up genetic transformations in subproblems ready for dissection. I believe that this analytical approach, which slices bioethical problems into slivers, is positive because it allows careful examination of the sum of the problems contained in bioethics, and likewise reaches a critical theory that appears to be unified. This method aims to offer a number of possible responses to problems related to bio-engineering Life and to link philosophical explanations to the above mentioned problem (biotechnology).

l will use the organic unity model as a moral reference to discuss the ethics of engineering Life. Clearly to do so we first have to answer this question: 'To what extent should the organic unity model be used to discuss the ethics of human and animal genetics?'

The organic unity theory can be used as a reference in ethics if we agree that living beings have a minimal value in themselves as existing biological entities ${ }^{4}$. This does not mean that here $I$ am proposing a new version of biocentric ethics, à la Naess. I simply propose that we may attribute a minimal value to organic systems and then apply this concept to the organic unity theory ${ }^{5}$. This would involve that the moral value of a given organism varies during the course of its existence depending on its degree of complexity as an organic unity. In the previous section of this dissertation we have seen that the growing complexity of the biological-psychological features of an organism increases during the course of its Life. Such an increase reflects the complexity of the organic unity of an organism. As the complexity-degree of an organic unity varies trough time (the degree of a human pre-embryo cannot be equated with the one of a foetus), such a degree differentiates the moral value of different living entities (speciesspecific individuals). The organic unity theory then, gives us a moral 
taxonomy.

As we will see in Chapter II.4 (II.4.5), in this dissertation I will advocate a moral difference between human beings and other animal species. According to the organic unity model, human beings have a predominant value within a moral taxonomy because they have a higher degree of organic unity complexity (biological and psychological complexity). This predominance is also reinforced by socio-cultural capacities that make de facto human beings the 'moral community makers'. This assumption will differentiate my conclusions about the ethical legitimacy of genetically modifying human beings (human inheritable gene modifications -chapter II.3) or producing genetically modified animals (transgenic animals chapter II.4), as well as producing human clones (chapter I1.6) or animal clones (chapter II.5).

A corollary argument of the above mentioned claim is that if we attribute a minimal value to living organisms as existing biological entities, then the interaction 'from the outside' with their biological existence becomes problematic from both a biological and ethical point of view. This line of reasoning makes the organic unity model relevant for both ethics and theoretical biology. In fact, if organisms are organic units whose existencepersistence refers to mechanisms that unify the biological-psychological diversity into a unitary diachronic entity, the organic unity model can provide a strong conceptual platform to approach the ethical issues of bioengineering. We will see that those applications of bio-engineering that do involve a bifurcation in the continuative existence of a (GM) organism do have a negative ethical connotation according not only to both the organic unity theory the closest continuer schema as well. According to my organic unity theory, those application of biotechnology that do not involve a profound interaction with the organic mechanisms of identity, such as cloning, do not posit substantial ethical problems.

\section{NOTES}

I In previous section of this dissertation we have seen that we still have a superficial knowledge of countless biological phenomena, such as; mechanism of DNA's phenolypic expression, population genetics, evolutionary mechanisms, biotic balances, and so on (Rothman 1999). These heuristic limits refer to numberless factors, such as: the limits of non-linear mathematical models in population genetics, the synergetic nature of genotype expression in phenotype, the absence of a unified theory on natural evolution, the inllueuce of environmental factors in gene 
expressions, and so on. All these limits do not allow us to predict lang-term consequences of engineering Life.

2 "It would be irrationally self-defeating if we decline to permit work which is no way immoral and which can benefit us all, merely because we fear that at some future time we will not have the courage to object to work that is immoral." Harris J, 1985, p. 127

${ }^{3}$ The interdisciplinary nature of bioethics often causes a paradoxical phenomenon in which bioethics research, instead of being enriched by the use of different disciplines, utilises superficially and a-critically some of them. Economic factors (the cost-benefits model), societal factors (from the job opportunity to the public perception), or biological factors (from tunctional food, to therapeutic use of GM food products) or ecological factors (from environmental impacts, to interspecies balances), have been stressed as 'ethical implications' arising from the use of biotechnology. Why? Why should we consider such arguments as relevant from an ethical point of view? If the link between 'problem implications' to 'ethical implications' is not explained, people have the right to refute these arguments. Or better, they can simply consider them as 'problems' which do not pertain to ethics.

${ }^{4}$ This assumption refers to intuitive factors. We all should agree that a dog has a different 'value' than a stone.

${ }^{5}$ One may argument that the ethical relevance of identity is an inferential argument to justify my use of the organic unity theory in bioethics. I do not agree wit this claim. Let us consider an example. In the chapter on fluman reproductive cloning we will see that a number of experintents to create human hybrid are taking place around the world. If we create a perfectly healthy organism (say a human-cat hybrid), why should we consider this use of biotechnology unethical? Let us suppose that such a manipulation is safe and effective and human-cat hybrids have better adaptive capacity than Homo sapiens do. If we use the $\mathrm{C} / \mathrm{B}$ model to say whether this use of biotechnology is ethical, we should say that that is an example of how humans can take the control of the Natural evolution. But I do suppose that most of people would not think of the human-cat hybrid as a positive goal achieved by biotechnology. Why? To me this is because we do attribute a value to human beings as well as other non-human living beings as existing living entities. In this sense, both the individual specific and the species-specific identity are considered ethically relevant. 


\section{III.2. Genetics' Dreams.}

\begin{abstract}
"(...) the possibility of controlling our children's" genetic destiny strikes me as only good. It is grossly unfair that some families' lives are dominated by the horrors of genetic discase" (Watson 1998:86)
\end{abstract}

In 1998, the pioneer of gene therapy, F. Anderson, has submitted to the US National Institutes of Health (NIH) Recombinant DNA Advisory Committee (RAC) an application to discuss protocols for in utero gene therapy to treat two pathologies: homozygous $\alpha$-thalassaemia and severe combined immunodeficency caused by lack of the enzyme adenosine deaminase (ADA). This technique would involve a change of the futureborn individual in situ, and has launched discussions about the ethical implications surrounding the gene manipulation of human germ cells in both Europe and US. The above mentioned technology is available and may be (potentially) a treatment for patients suffering of lethal diseases (Zanjani \& Anderson 1999:2084-2087). This is an example that problematic implications arising from bioengineering need to be seriously analysed in their own complexity (Wadman 1998:420; Couzin 1998:27, Gordon 1999:2023-2024). And 'serious analyses' means that we have to explore both the medical potentialities/limits of such a technique and its ethical implications (Cohen 2000). Recent discoveries would facilitate the use of biotechnology to modify human germ cells (Dasgupta 1999:16-17; Vogel 1999:1432-1434). For example, the culturing of human embryonic stem (ES) cells ${ }^{1}$ will offer to scientists the possibility to better study embryogenetic processes, or to treat (probably) some specific diseases. For example, ES cells might be used to treat Parkinson's disease, juvenile-onset diabetes. (Thomson 1998:1145-1147).

Having this in mind let us explore both the state-of-the-art of gene therapy and consequences of genetic determinism that have dominated the scientific community during the last 20 years. But first, I wish to say that in this dissertation I will use the term Human Inheritable Germ Line Gene Modification (HIGM) rather than germ line gene therapy (GLT) because, as Frankel and Chapman have correctly stressed (AAAS Report, September 2000), the term 'therapy' for gene modifications of germ 
cells is sometime reductive and misleading ${ }^{2}$. The inheritable gene modifications may be performed for both therapeutic and nontherapeutic (enhancement) purposes. Or some applications of somatic gene therapy may cause inheritable gene modifications (Cohen et al. (1997) pp. 186-87 and Rubenstein et al. (1995) pp.316-39). Thus, if we limit our analysis to therapeutic applications of HIGM we risk forgetting all those uses of human genetics performed in hope of increasing the biological functionality of human beings.

\subsection{Is Gene Therapy the Future of Biotechnology?}

Recent data say that $3 \%$ of live-bom babies have significant birth congenital anomalies. $10 \%$ of these anomalies have a genetic cause (Billings 1999). Genetic pathology is the cause of $30-60 \%$ paediatric hospital admission in developed countries (Baird 1988; de Capua, 1992). Only $15 \%$ of people with mono-factorial diseases can increase the length of their life through non-genetic therapy.

In order to 'correct' gene anomalies, geneticists have improved techniques and are now able to replace defective genes with a non-defective corresponding nucleic acid sequence. Such gene transformations can be used to 'transform' a genome in a pre-ordinate way (bioreactors, transgenic organisms, chimeras, and so on), or to treat a pathology (this means to use genetics as a therapy). Gene therapy is the insertion of normal or genetically altered gene into the cell nucleus. Potential goals of gene therapy are: 1) add a missing gene; 2) replace a defective gene (defective genes are substituted with a non-defective corresponding nucleic acid sequence); 3) block translation of defective gene.

There are two current kinds of gene therapy: somatic gene therapy, and germline gene therapy. The first treatment is applied to somatic cells, the second to germ cells. The basic difference between these two applications is that somatic gene therapy transforms a pathological nucleus sequence into non-defective sequence. This genetic manipulation is not transmitted to a patient's progeny. Somatic therapy (GT) is a treatment 'for' the patient, where germline therapy (GLT) is a treatment, which transforms the individual's gene identity (Walters 1999:16-17): the gene manipulation will be expressed in all individual's tissues. Descendants will inherit this manipulation. Current limits of gene therapy are: 1) Difficulty of controlling vectors of the bioengineered gene (vectors used are: adenovirous, adeno- 
associated virus or liposomes); 2) Difficulty to predict the gene expression of the bioengineered gene; 3) Impossibility to treat polygenic diseases to date.

At the present, the best method to introduce the genetically modified exogenous DNA in an organism is the use of viral methods (retrovirus, adenovirous, adeno-associated virus, vaccina virus, poliovirus, and so on -Mulligan 1993). The fundamental difficulty that gene therapy needs to resolve is not the transformation of nucleus sequences, but planting the correct gene in the right locus (Smaglick 1999:9). For example, retroviruses are vectors with which the corrected gene is planted, but this is done with little possibility of controlling the position that this sequence will have in the nuclear structure ${ }^{3}$.

Somatic gene therapy has been an approved clinical trial for cancer since 1990 (TILs gene, causing necrosis factor -Friedmann 1999). On September 14, 1990 researchers at the U.S. National Institutes of Health performed the first approved gene therapy procedure on four-year old Ashanti DeSilva. Born with a rare genetic disease called severe combined immune deficiency (SCID), Mrs DeSilva lacked a healthy immune system, and was vulnerable to every passing germ. The idea was that biotechnology could restore a biological mechanism that had some dysfunctions: 'direct' (let us think of monogenic dominant diseases such as Huntingdon's) or 'potential' (let us think of AIDS, oncogene) genetic defectiveness. These dysfunctions were seen as 'directly' or 'indirectly' determined by defective genes (Friedmann 1999).

In 1998, Nobel Prize laureate Watson, said that "the possibility of controlling our children's' genetic destiny strikes me as only good. It is grossly unfair that some families' lives are dominated by the horrors of genetic disease" (Watson 1998:86). This position does not reach a global consensus among biologists. As a matter of fact, gene therapy, while being a promising technique, has not achieved the expected scientific goals yet (Dutton 2000:1-11). The multiple relationship among genes, disease proteins and the immune system (only to quote some factors) have shown the impossibility to reduce genetic diseases to simple causative factors to be simply 'substituted' by non-defective corresponding nucleic acid sequence. And the death of a 18-year-old patient on September 17, 1999, has lunched serious discussions among biologists ${ }^{4}$ about both the safety of gene therapy and its effectiveness ${ }^{5}$ (Bardour 2000:384). 
Regardless of the few positive results achieved by gene therapy in the last 10 years and the current crisis of genetic reductionism (see chapter 1.2), the claim that through gene therapy scientists may enhance humankind, in terms of health or physiological potentialities, has consolidated among scientists. Glenn McGee (1997) said; "it is easy to imagine a world 100 years from now in which parents have much more control over the inheritance of children. Gene therapy and pre-implantation diagnosis for 6-8 cell embryos will be thoroughly assimilated into obstetric technologies." But, is it true that human society has, in its grasp, the possibility of controlling future children's' biological features? Let us explore the heuristic limits of human genetics, in particular the claim that it is possible to manipulate human beings' germ cells in a pre-ordinate way (Gordon 1999).

Human germline gene manipulation has not yet been intentionally fulfilled at the present, but it may use the same techniques as somatic gene therapy or animal experimentation (transgenic animals, chimeras) or new technologies that may be developed in the future (in womb gene therapy, insertion of artificial chromosomes). Potentially, it might be used to treat pathologies currently causing the death of millions of people. For example, in 1983,242 millions of people suffered from $\beta$ thalassemy. Is it reasonable to claim that gene manipulation may treat these people?

First, we need to distinguish between homozygous and heterozygous people. A homozygous person with, for example, sickle cell anaemia has a lethal disease, but heterozygous people with the same anaemia are more resistant to malaria. In different countries, the presence of anaemia alleles is a tool for developing biological resistance to various pathologies (Macer 1990, Rottler \& Diamond 1987). If we compare the percentages of people with a genetic anomaly we see that, for example, in Central Africa 2,5-8\% people have anaemia, but that in North America this percentage is only 0,13-1,3\% (other differences can be looked at in Haemophilia influence B, Thay Sach's Syndrome, Diabetes, and so on). There are phenomena of population genetic compensations that increase the individuals' resistance to particular pathologies. Knowing this, how can we decide which gene abnormalities must be corrected to fit with a human being's genome? Is it actually possible to eliminate these pathologies on a population scale?

According to biology, the answer to this last question is a resounding no! Mutational phenomena and gene polymorphism tell us that pathologies are highly diversified. (This being the case, it becomes very difficult to define an ideal model of a 'bad' gene.) In parallel, environmental and behavioural factors continuously influence the pathological expression of a potential 
genetic disease ${ }^{6}$. When we think of the continuous transformations of pathogenic agents, we see that it is impossible to totally eliminate diseases that are caused by alleles. Thus when we think of gene manipulation as a treatment for most of the lethal diseases affecting human society, we must be aware of three factors:

- Pathologies causing millions of deaths in today's society do not entirely refer to genctic diseases (such as: hypercholesterol, hepatitis, AIDS, and so on);

- We cannot treat polygenic diseases through genetic manipulation since polygenic diseases do not refer to a single gene's dysfunction (Peltonen 2001:1226-1227). When we interact with the equilibrium among genes, we cannot predict whether this action will detcrmine positive or negative outcomes. Both the phenotype expression and subsequent mutations of the bio-engineered gene may determine physiological dysfunctions;

- The incidence of genetically enhanced gene lineages towards gene population mechanisms (gene frequencies) would not be changed via gene manipulations of man since both changes in allelic frequencies resulting from selective pressure and sexual reproduction mechanisms would obstruct the human attempt to biologically enhance human race (Gordon 1999).

\subsection{Dreams of Genetics.}

Some experts (Harris 1994, 1992; Glover 1984) claimed that a possible scientific goal of gene manipulations would be an enhancement of the genetically modified organisms. However, when we consider possible artificial manipulations of a genome performed in the hope of increasing the biological functionality of an organism, we have to remind ourselves that human knowledge on both genomics and phenotype expression of polygenic characters remains superficial and to a certain extent, naïve (Rothman 1999).

Let's consider a practical example: onco-genes. I have already explained that tumoral pathologies are not necessarily linked to a specific gene deficiency (chapter I.2), but rather they refer to a dynamic and synergetic process. Peculiarities of onco-genes and suppressor genes are still unknown. We thus do not know, or cannot predict, whether germline gene manipulations would be a useful treatment for cancer ${ }^{7}$. By the same token, we cannot know whether gene manipulations intended to increase immunoresponses to oncogenes will be an appropriate instrument for contrasting 
tumoral phenomena. Thus, the claim that gene manipulation may enhance immuno factors of individuals weakens due to the state-of-the-art of human genetics.

Also other potential applications of bioengineering (e.g. LeshNyhan syndrome, Cystic Fibrosis, Duchenne's muscular dystrophy, Haemophilia A) should be deeply analysed by taking into account their effectiveness. If one has, for example, a double couple of a gene that is causing a deficiency in his-her haemoglobin production, and we suppose that biotechnology would be able to plant the 'spliced' gene in the right locus of his-her genome, we strive only to correct a gene defectiveness. However, if a person has only a single couple of this 'bad' gene, we can then treat this person only if we suppose that this gene's transformation is relevant in efforts to decrease the transmission of this gene in future generations. But 'that' person is not sick! And his/her genetic 'abnormality' may result in a positive manner for other biological immunologies (Mediterranean anaemia offers a sharp example). For example, if we consider the heterozygote form of adrenal hyperplasia in a recessive gene (Macer 1990:303) we see that Yupic Eskimos are protected from haemophilus influencae B. Other 'diseases' have been employed as biological tools for preserving people against other pathologies. So it is difficult to consider the role of homozygote people with diseases that are only in a potential form.

All these example refer to a common problem: when we think of gene manipulation as a treatment we suppose that: 1) we have the possibility of controlling the phenotypic expression of the required characters, 2) it is possible to draw a line by which a gene transformation can be accepted or refuted. Both these claims are debatable and need to be explored in detail (Smaglick \& Rayal 1999, Verlier 1999:31-35). We have seen that we cannot actually foresee the potential long-term consequences of a large-scale genetic repair (the consolidation of the artificial character in a given population). But, even if we assume that we can take this risk, and that in a future we will be able to solve the technical problems of controlling vectors, how can we draw a line between pathological and non-pathological genetic determinants?

\subsection{Treating or Enhancing?}

When we consider legitimate the use of a gene manipulation performed to correct a genetic abnormality ${ }^{8}$, we have to be aware that such 
a claim, if coherently applied in human genetics, may cause problem situations. Let us consider two consequences of this view: the growth hormone, and cosmetic applications.

Children suffering from Growth hormone-deficiency (GOD) can be treated by somatotropine. Somatotropine was produced in 1979 by Genetek, and is now used to treat people with a growth hormone-deficiency. Those who consider ipofisario nanism as a pathology could claim the necessity to use human genetics to 'treat' those individuals whom taller is down the taller average in a given country. For example each year in the US, $3 \%$ of new born babies -90.000 in 1995-are down the taller average. We might say that we should use human genetics to treat ipofisario nanism because our action has a 'potentially therapeutic' value, since it can be hypothesised that there is an association between shortness and psychological morbidity (Daniels 1992:46-48, White 1993:401-409, Tauer 1995:18-20). Therefore, on the one hand, we cannot say that a 'small' person is sick, on the other hand if we claim that gene manipulations should not refer only to pathologies we also should accept all of the 'wishes' of those involved. Then we should support the legitimacy of public health policies based on a complete liberal approach to human genetics. According to this, patients would have the 'right' to choose whether their genetic makeup should be modified to satisfy their expectancies, regardless of the content of these wishes. Cosmetic applications of gene manipulations should be allowed as well. Would it be reasonable to allow cosmetic applications of gene manipulations? Do these applications have a so strong legitimacy to justify the human interaction with the Natural evolution? Is it really possible to draw a line between therapy and enhancement?

Take the case of melanoma. Melanoma is an epithelial cancer caused by ultra-violet radiations of solar light. Since the quantity of ozone in the atmosphere is decreasing, the quantity of ultra-violet radiation is continuously intensifying. In parallel, the number of melanoma pathologies is increasing and physicians predict the incidence of this disease will grow over time. Since this pathology refers to the quantity of melamine in the skin (melamine protects epithelial tissue from these radiations), we could suppose that if we artificially increase the quantity of melamine in a person, his/her possibility of having this pathology will decrease. According to this, a radical solution would be to 'make' all humans 'black.' In this case, we would achieve two positive results: (a) the end of racial prejudice and (b) a reduction of the incidence of that pathology in a population (Salvi 1996). Can we accept this argument to promote such a health policy? 
We can apply this claim to discussions surrounding the hypercholesterol level in human beings. Since the metabolic control of fats can determine cardio-vascular diseases if we transformed those genes that are involved in the metabolism of fats we could achieve two goals: 1) we would decrease the incidence of cardio-vascular diseases at a population level; 2) we would increase the quality of life of those people who undergo these transformations (in a cosmetic, sexual, and social sense).

The examples above are deliberately extreme. They show that we may justify a number of really debatable gene manipulations by claiming their therapeutic goals. Scientists may justify many changes of people's germ cells by stressing the (pseudo) therapeutic ends of such gene manipulations. Nevertheless, regardless of the effectiveness of these manipulations, it is a fact that these scientific goals are relative. They refer to biological and socio-cultural factors. As such, why should gene manipulations be performed to achieve these goals? Why should we think of enhancing the human species when we have evidences that this attempt is without scientific foundation'? When an 'artificial' gene trait is transmitted to progeny there is no way to end the transmission of this factor through time. The manipulation is irreversible. Future generations will inherit this element, even if it is not effective and it does not respond to the needs of future born people. (For example in a future some functional food will be produced with a low rate of fats, or it will be possible to filter ultra-violet radiations.)

Similar considerations can be done in thinking of OCA albinism, tyrosine albinism, and XYY syndrome. All these diseases could be considered "individuals' abnormalities" (Caplan 1992). Clearly the disease-asabnormality concept refers to social factors. Can we say that in those cases in which abnormal features of an individual do not cause significant physiological dysfunctions 'for' him/her, we should modify his/her genotype? How can we deny that our normality-concept depends on social and cultural factors?

\subsection{Bioengineering and Public Health Policy.}

As I have already said, some experts have claimed that human inheritable germ line gene modifications (HIGM) may eliminate diseases, which are currently causing millions of deaths in the third world. For example, $\beta$ thalassaemia, the most common lethal genetic disease, could be altered at the population level. In doing this, we would decrease the allelic 
frequencies of this pathogenic gene. (We can apply this idea to pathologies that have not been defeated by vaccination policies). Why do we tend to obstruct similarly positive results?

I have already said that HIGM may influence the dynamics of population genetics. Consequently, we could think of biotechnology as a weapon with which to fight mortal diseases that are killing millions of people. Germline gene manipulations, thus, could be seen as ideal tools for eugenic policies. Would such an use of human genetics be unethical? [It is not my intention to analyse the history of eugenics ${ }^{9}$. I only aim at underlining that eugenics is a problematic topic (let us think about nazi eugenics or Soviet-Russian and American programmes of sterilisation for treating pathologies (Lewis 1986; Lewontin 1982, Moreno 1999, Seife 1999).]

Eugenic policies have been adopted in many 'civilised' countries (Moreno 1999). For example, the government of Singapore instituted numerous eugenic policies during the 1980 s including a policy of providing financial incentives to 'smart' people to have more babies. The California-based Repository for Germinal Choice, known more colloquially as the Nobel Prize spermbank, has assigned itself the mission of seeking out and storing gametes from men selected for their scientific, athletic or entrepreneurial acumen. Their sperm is made available for use by women of high intelligence for the express purpose of creating genetically superior children who can improve the long-term happiness and stability of human society (Caplan 1992). Selective abortion could be seen as one example of eugenic application (McGee 1998:245-249). So, if we seriously want to investigate the implications of such gene manipulations, we need to face eugenics without prejudice. Even if the 'idea' of eugenics is connoted in a common sense as 'wrong', organisms resulting from eugenic programmes are accepted daily by people (think of your dog or the meat you cooked yesterday).

As I have said, germline gene manipulation may be a tool for eugenics. We could apply this technique to treat pathologies or potential pathologies or we could utilise it to improve the consolidation of a particular (arbitrary) character within a population. But, is there a proof that such hypothetical use of bioengineering is possible?

The conceptual platform of this hypothesis refers to the heredity mechanism. If a genetic disease is transmitted to progeny, then "pathogenic (pools of) genes' will have a higher incidence in population genetics. balances. If we modify these pathogenic genes, we might decrease the frequency of these genes in a given population. This claim has been the 
milestone of eugenic policies. Nevertheless, mutational phenomena and polygenic diseases weaken this argument. Genetic drift mechanisms, crossing over phenomena, mutations, or simple biological variables make it extremely difficult to achieve the goal of interacting with genetic mechanisms at population leyels. When we pass from an individual specific level to a species-specific level, we see that eugenic applications of germline gene manipulations performed to consolidate a particular allelic frequency in a population, are utopian goals (Gordon 1999). We do not know if the 'desired' character will change through time by subsequent mutations, or if it will consolidate in a population. How can we decide to consolidate a specific pool of genes at a population level if changes in the allelic frequencies determined by sexual reproduction and environmental factors will obstruct this goal? How can we define 'bad' and 'good' genes? These questions rely on the meaning of pathology and the relationship between pathology and genome.

\subsection{Consequences of Genetic Determinism in Human Genetics.}

One of the crucial elements to be explored in human genetics is the concept of genetic disease. This is because, when we hypothesise that germline gene manipulations are 'treatments,' it becomes obvious that the notion of genetic disease reaches a consensus among biologists. Surprisingly, a standard-sense of this concept does not exist. We have two basic approaches to the genetic disease concept: the reductionist and nonreductionistic approaches. According to the first approach, a genetic disease is:

1) A single locus defect, with $100 \%$ heritability (Huntingdon's disease, Phenylketonuria -PKU- Sickle-cell anaemia);

2) A genetic predisposition -even if less than $100 \%$ heritable- which cocause the disease (heart disease, some forms of cancer, diabetes, etc. are considered 'genetic disease');

3) A genetic predisposition to complex behaviours causing a disease (alcoholism, for example).

On the contrary, the non-reductionistic approach to the concept of genetic disease underlines the active role of environmental, social, behavioural and psychological factors (Magnus 1997). If we consider genes to be the determinant factors of living beings' existence, we may deduct that all 
pathologies result from a genetic abnormality. In this view, when we study a particular pathology we hope to find the causal relation between genes and their (pathologic) phenotypic expressions. We do not think of pathology as a 'process,' but rather, we consider it a mechanic 'expression' of a gene's defectiveness (chapter I.2). Can pathology be considered a mechanic process?

Let us first face the problem of a pathological gene model. Human beings have a pool of 3 billion nucleotides. If we seriously compare different peoples' genomes we see that, on average, 3-5 nucleotide differences exist between two couples of the same gene. Even if we suppose that it is possible to define an ideal model of a 'healthy' gene, and another of an 'unhealthy' gene, how can we know what differences are responsible for the expression of a given pathology? Also, if we consider different people with the same pathology, the single nucleotide polymorphisms (SNPs) and the individual phenotypic variation decrease the possibility of defining an ideal model of pathology. If we seriously think of applying gene manipulations to treat diseases in populations, we would need a model of a 'good' gene to restore the non-pathological structure of a nucleus sequences. This model, however, exists only for a limited number of genetic diseases (monogenic dominant diseases) its validity is strongly affected by both SNP and the metabolic pathway causing a disease. And it does not refer to both polygenic and multifactorial diseases.

The problem is that genetic reductionism thinks of disease as a stable phenomenon of Life. When we think of pathologies we consider their common symptoms as an exhaustive element that explains their nature to us. But this is misleading. If taxonomic criteria classify diseases through their internal similarities, the pathological expressions of those diseases will refer to individual factors and peculiarities. Diseases are also contextdependent ${ }^{10}$. We cannot simplify the concept of disease by saying that (for example) melanoma is 'that' disease because its genetic determinant has a margin of structural similarity high enough for doctors to identify it with the melanoma's gene model. The individual specific melanoma's expression will indeed be similar, but not identical to the ideal model. We can apply this concept to causal elements, which determine pathology. Take a set of causes, such as; behaviour, life style, diet, stress, and so on. All of these elements have an active role in the expression of a disease. If we think that pathology can be reduced to a specific determinant, we have to highlight that this is only one component of pathogenic factors. It is a piece of the puzzle, not the whole picture. This is also true for monogenic diseases, such 
as cystic fibrosis. In fact, recent discoveries show that the clinical phenotype expression of the disease is actually resulting from multiple genetic determinants (see Dipple K. and McCabe R. (2000) Molecular Genetic Metabolism 71, 43).

When we follow a reductionistic view, we need a 'genetic' cause, which has seemingly sparked the growth of the pathology. Clearly, when we realise that a disease results from a number of heterogeneous and multiple causes, this mechanistic view collapses.

To the contrary if we think of Life through gene reductionism we would claim opposite conclusions. According to gene reductionism, we find all human features in genes. Homosexuality in this instance would be seen as a genetic abnormality. Intelligence, likewise, would be considered as determined by specific gene structures. (This hypothesis has been refuted by the study of many biologists (Hammer, Magnus, Patatucci, 1983:321-327.) Luck would in this case also be thought of as a genetic predisposition (Sid 1998), and so on. If this were the case, we would look for a direct causal correspondence between genes and human features. But this claim is simply biologically false and refers to a cultural paradigm that has dominated the scientific thought for the last two decades (chapter I.2). According to results emerging from the sequence of the human genome published in Science on February 16 2001, we have to avoid using the gene reductionism. This is because the primary DNA sequence of a gene is not in itself sufficient to predict its functions in the whole organism. Contrary to genetic mechanistics, the complexity of the biological nature of human beings has to be explained through the interactive nature of genes rather than the structural complexity of the human genomes. The difference of complexity among organisms belonging to different species refers to the complex gene regulatory networks that govern cellular physiology, and differentiation and development of the organism. Therefore to understand the molecular basis of diseases we need information about gene expression, when and where the corresponding protein performs its functions (proteomics) and how the functions of a protein and its interactions with other molecules can be predicted from its three dimensional structure (structural genomics). Functional-genomics approaches (DNA-arrays, proteomics, structural genomics, comparative genomics and bioinformatics) are being developed to increase fundamental knowledge on the functions of these genes and the potential links to disease. This means that the combinatory nature of genetic code is now strongly supported by the human genome mapping. The implications of such a discovery towards bio-medical research is that we 
have to focus our attention on the relationship the environment and the individual and within an individual's constituents (interactive system) rather than thinking of diseases as merely caused by genetic failures (a genetic defectiveness for each human disease). According to Dr Peltonen and Dr McKusick we may summarise the consequences of the human genome sequencing towards bio-medical sciences as follow (Science 2001 (291):1226).

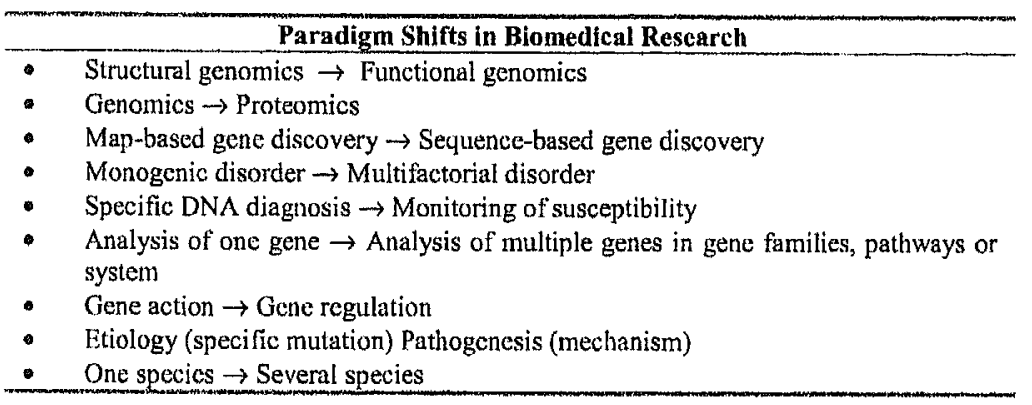

Eugenic policy, thus, refers to a 'wrong' disease-concept based on genetic reductionism. If we refute this approach, we ought to refute eugenic policies since its therapeutic goals cannot be achieved. We can defend a similar conclusion for 'potential' pathologies as well. When we suppose that it is possible to enhance the organisms' biological response (immunity systems) to pathological factors (HIV, cancer, etc.), we forget the contextual dependence and synergetic nature of these pathologies. The impossibility of defining an ideal model of 'healthy' nucleus sequence, to interact with polygenic diseases (or intra-genes signals), to control the phenotypic expression of a genetically modified (exogenous) gene, and the interactive nature of genes make it, both conceptually and pragmatically, difficult to think of germline gene manipulation as a promising technology to be adopted in public health policies.

In parallel, when germline gene manipulation is constructed with respect to therapeutic ends, we have to distinguish between 'direct' and 'indirect' gene dysfunctions and between 'polygenic' and 'monogenic' diseases. When we think of gene manipulation as a way to substitute a defective nucleus sequence, we aim to restore the structure of a defective gene. In polygenic diseases we do not have a 'broken' nucleus sequence but an alteration of the equilibrium among genes. This anomaly causes pathogenic results. In this 
case, a gene manipulation cannot treat a patient, because he/she does not have a structural anomaly in his/her genome, but rather an anomaly of the global equilibrium among genetic signals producing proteins. The synergetic nature of diseases limits the possibility to predict consequences of an external transformation of this equilibrium, or the gene expression of this transformation ${ }^{11}$. When we emphasise the differences within the expression of a given pathology, the lack of possibilities to reduce the human genome to an ideal model, the genetic polymorphism, and the individual phenotypic variation, then the arguments to support gene therapy for therapeutic and not-therapeutic purposes collapse.

\subsection{Conclusion.}

In 1991 Dr Eric Juengst summarised the arguments in favour of human germline gene therapy (GLT) as follows: "1) GLT offers a true cure, and not simply palliative or symptomatic treatment; 2) GLT may be the only effective way of addressing some genetic diseases; 3) by preventing the transmission of disease genes, the expense and risk of somatic cell therapy for multiple generations is avoided; 4) medicine should respond to the reproductive health needs of prospective parents at risk for transmitting serious genetic diseases; and 5) the scientific community has a right to free inquiry, within the bounds of acceptable human research." (Juengst, 1991, p. $588-89$, quoted in the Scope Note 24 of the US NRCBL). As we have seen, these really promising goals can hardly achieve the expected results. We need to be aware that the germline transformation has a number of practical limits:

- its application to enhance humans is biologically nonsensical (both at an individual and species level);

- if we cannot predict the phenotypic expression of bioengineered genes, then we cannot say whether such a gene manipulation may treat a patient;

- its applications to promote eugenics policy (positive or negative eugenics) are obstructed by biological population genetics mechanisms, such as: genetic drift, mutational phenomena, environmental pressures, gene polymorphism, and so on.

When we underline the practical limits of gene manipulations, the first impression that hits is: 'how can we think of this technique as a controllable 
tool to improve the human race?' 'Enhancing Life', is a myth of human culture. The possibility to interact with the biological matrix of humans or animals revolutionises the role of human beings in Nature: from the human being as a 'passive object' of natural processes, to the human being as an active actor of the Natural evolution (Salvi 1995). However, this revolutionary role of human beings has to be related to the concrete possibility to achieve the expected scientific goals. At the present, we cannot control and interact (in a pre-ordinate way) with mechanisms of population genetics. We do not know how the expression of polygenic characters works, what is the role of non-coding sequences in genomics, and the way how scientists look at DNA in biology is changing, etc. And the mapping of human genome is addressing bio-medical research to explore proteomics and chemical interactions among genes. How can we suppose enhance or treat human beings through bioengineering if we do not understand so far the nature of human genetic makeup?

\section{NOTES}

1 "Stem cells are capable of continually reproducing themselves and serve to renew tissue throughout an individual's life. (...) the most fundamental and extraordinary of the stem cells are found in the early stage embryo. It is the potentially unique versatility of the ES [embryonic stem] and EG [embryonic germ] cells derived, respectively, from the early stage embryo and cadaveric fetal tissue that presents such unusual scientific and therapeutic promise. Indeed, scientists have long recognized the possibility of using such cells to generate more specialized cells or tissue, which could allow the generation of new cells to be used to treat injuries or diseases, such as Alzheimer's disease, Parkinson's disease, heart disease, and kidney failure. Likewise, scientists regard these cells as an important perhaps essential - means for understanding the earliest stages of human development and as an important tool in the development of life-saving drugs and cell-replacement therapies to treat disorders caused by early cell death or impairment." National Advisory Bioethics Commission (US) Ethical Issues in human stem cells research, September 1999

2 "One form of [inheritable genetic modification (IGM)] would be to treat the germ or reproductive cells that develop into the egg or sperm of a developing organism and transmit its heritable characteristics. Another form of germ line therapy would be to modify the gametes (sperm and egg cells) or the cells from which they are de-rived. Still other technologies under development, such as the insertion of artificial chromosomes, would also introduce inheritable genctic changes. Greater knowledge of genetics is also making it possible to con-template genctic interventions not only to treat or eliminate diseases but also to "enhance" normal human characteristics beyond what is necessary to sustain or restore good health. Examples would be efforts to improve height or intelligence or to intervene to change certain characteristics, such as the color of one's cyes or hair. Such interventions could be attempted through either somatic moditication or IGM." A.AAS, September 2000, p. 10 


\footnotetext{
${ }^{3}$ If $a$ virus is planted in the wrong locus of a patient's genome functional deficits or neoplastic processes may result. Then, at the present we cannot say that this technique is safe (Marshall, 1999:2244-2245). But we do know that the bioengineered gene may be transmitted to descendants, which would lead to variations in the genetic makeup of future generations (Smaglick \& Rayal 1999). According to this, on the one hand we will be not able to predict whether the gene manipulation will produce a possible expression of the desired character or will cause a cascade of events determining a gene dysfunction (for example a denaturation of a specific enzymatic production) or subsequent mutations (Gordon 1999:2023-2024). On the other hand, we know that this gene alteration will be phenotipically expressed, will influence the organism's biological individuality and may provoke discrepancies in generational gene changes.

${ }^{4}$ In the scope paper prepared by the National reference Centre for Bioethics Literature the death of J. Getsinger is described as follow: "In October 1999, the death of Jesse Gelsinger, the first fatality in a gene therapy experiment, was reported in Nature (Lehrman 1999). Subsequen investigations revealed that the deaths of six gene therapy patients had not received the usual public disclosure that has characterized gene therapy research (Nelson and Weiss 1999) Gelsinger's death also raised questions about researcher entrepreneurial activities and conflictof-interest, and about government oversight procedures". (Nelson and Weiss 1999). On this topic let us see http://www.georgetown.edu/research/nrcbl/scopenotes/sn24.htm

severtheless, gene therapy is still considered as a potential candidate to treat a number of genetic diseases: severe combined imnunodeficency caused by lack of the enzyme adenosine deaminase (ADA), and 'severe common immune deficiency' (SCID), or, in a fiture, syndrome of Lesh-Nyhan, Cystic Fibrosis; Duchenne's muscular dystrophy, Haemophilia A, homozygous a-thalassaemia, HIV and cancer

${ }^{6}$ The consequences of the human genome sequencing with regard to both genomics and medicine is analysed in Peltone, L. and McKusick V. A. 'Dissecting human discase in the postgenomic era', Science (291), 16 February 2001, pp. 1224-1229

Cancer is a 'complex network of mutually dependent causative factors $(.$.$) it is becoming$ increasiling clear that the genetic basis of human cancer is combinatorial' (Szallai 1998:1292 1293)

${ }^{8}$ A problematic case of "potential disease" is the set of pathologies which is manifested in up in years, Let us think of the Huntigton Corea disease. This pathology refers to an anomaly of the chromosome 4. This neuro-logical pathology is manifested only after the 30 th. years, and causes senile dementia. Huntigton Corea disease is a dominant character, also heterozygous people will be sick. We can hypothesis to apply a human Inheritable Germ Line Gene Modification (HIGM) to treat this disease. This treatment is clearly preventive.

${ }^{9}$ First example oi eugenic law is the Swedish law -1757 - forbidding marriages betwcen people having endogen cpilepsy. The effort to increase the genetic incidence of some gene character at a population level is common in a number of countries. In 1996 the lion Kong government pushed woman to have children with western managers, thinking that future born babies would have inherited the business gene.

11 "No gene operate in a vacuum; rather, each gene busily interacts either directly or through its protein product with many other gene products. This results in marked variations in the symptoms of patients with the same discase. (...) Most common human diseases represent the culmination of lifelong interactions between our genome and the environment. (...) Many human diseases, such as hypcrtension, coronary artery disease and even some psychitric
} 
disorders, represent quantitative traits that are caused by interactions among genes and between genes and the environment." Peltonen L. and McKusick V.A. (2001):1226

"One may object that safety issues could also be claimed to oppose to monogenic dominant diseases. It is true that to date technical limits (control of gene vectors) obstruct gene manipulations perfomed in hope to treat both monogenic and polygenic diseases. These technical limits may be solved in a future. The problem is that polygenic diseases have a dysfunction of an interactive mechanism among a number of genes. Monogenic diseases have a structural anomaly of a specific gene's sequence. This defectiveness is easier to be identified. In polygenic diseases it is the 'mechanism' of intra-genes signals to be disturbed. As such, it is extremely difficult to say what determinant has caused this dysfunction. 


\title{
II.3. Shaping Individuality: Human Inheritable Germ Line Gene Modification
}

\author{
"The issues of germline therapy and enhancement \\ engineering need to be debated widely in society, \\ but arguments that genctic engineering might \\ someday be misused do not justify the needless \\ perpetuation of human suffering that would result \\ from an unnecessary delay in the clinical \\ application of this potentially powerful therapeutic \\ procedure" (Anderson 1984) \\ "Germline engineering would change every cell in \\ the body. Pcople would no longer have to make do \\ with haphazard combination of their parent's \\ genome. (...) Humanity would, in effect, take \\ control of its own evolution. (...) Evolution is \\ being superseded by technology, and the time scale \\ will be far more rapid. Humans are becoming the \\ objects of conscious design". (Taylor 1998:25-29)
}

At the present, no human germ line gene therapy has been reported, but Inheritable Germ Line Gene Modifications (HIGM) are already established and used on animals (Biotechnology News, 2.12.1999:2-3). The general attitude of Ethical Committees, institutional agencies, and social organisations expressed by several European countries is to recommend the prohibition of HIGM for ethical and scientific reasons ${ }^{1}$. We have abundant examples of this attitude. The Danish Council of Ethics underlined the eugenic potentialities of HIGM and the negative attitude of the Danish public opinion towards HIGM (Danish Council of Ethics Report 1987, 1988, 1989). This attitude has been confirmed in Germany, where the Parliament Commission of Inquiry has condemned HIGM because of its eugenic potentialities. In 1990, the Joint Ethical-Medical Committee of the Catholic Union expressed a similar conclusion because of the fear that HIGM would deny future generations' autonomy. The French and the Italian National Bioethics Committees both underlined the extreme problematic of HIGM (French Comité Consultatif National d'Ethique, 1986, 1990; Italian Comitato Nazionale per la Bioetica, 1991). However, biologists are considering the possibility of using HIGM in human genetics. 
A clear example of this attitude is the Declaration of Inuyama, which followed the Conference of the Council of International Organisation of Medical Sciences (CIOMS) in 1990. This document said that the modification of human germline for therapeutic or preventive purposes might be the only means of treating certain conditions. The debate is still open and new documents addressing issues related to HIGM are published on a regular basis. For example, in September 2000, the American Association for the Advancement of Science published a report on HIGM (Frankel \& Chapman 2000). The report stresses the need of having further discussions about HIGM and does not exclude a priori the ethical legitimacy of such a technology. In parallel, new scientific discoveries such us the production of artificial chromosomes and research involving embryonic stem cells ${ }^{2}$ (ES) open passionate discussions among biologists and ethicists about the ethical legitimacy of such an use of biotechnology ${ }^{3}$ (Cohen, 2000).

Here I will not explore issues related to potential scientific goals that may arise from the gene manipulation of human germ cells (see chapter II.2). I will focus on pro-cons arguments posited by bioethicists concerning the ethical legitimacy of HIGM.

\subsection{Bioethics and Human Inheritable Germ Line Gene Modification (HIGM)).}

Bioethicists have stressed a number of ethical implications of HIGM, namely; 1) the interests of future generations; 2) the human genetic integrity; 3) the safety issues; 4) the socio-economical implications; 5) the eugenic issues; 6) the human dignity. 1 have already dealt with some of them in the previous chapters of this dissertation (issues related to eugenics (II.2), therapeutic ends (II.2), gene determinism (I.1, I.2. I.3), personhood (I.7), etc.), here I will consider the above 6 statements under a conceptual point of view.

\section{The interests of unborn children}

As I have already said the main difference between somatic gene therapy and human inheritable germ line gene modification (HIGM) is that when we manipulate germ cells (a) the gene manipulation will be expressed in all the tissues of an organism; (b) descendants will inherit this manipulation. Does HIGM affect the interests of unborn children (Parfit 1984; Zoar 1991; Persson 1995)? We can argument that when we perform a 
germline gene manipulation for therapeutic purposes, we respect the interest of the unborn not to be sick as well as the interest of future generations not to inherit pathogenic gene traits. As such, HIGM would be ethically legitimate as a treatment. In opposition to this argument, we may also say that HIGM is not ethically legitimate because it does not respect the right of future-born individuals to inherit a non-manipulated genetic makeup and because there is a lack of consensus of the umborn. What is the right response to this dilemma?

Both the unborn patient undergoing an inheritable germ line gene modification and his/her future descendants cannot express a consensus and they will inherit a manipulated genome. However, in those cases in which the expected scientific goals of HIGM are clearly therapeutic (for example a treatment of a lethal dominant monogenetic disease), we have realistic reasons to assume that the unborn people have an interest into such a treatment (Harris 1994). As such, HIGM would be legitimate since we me may suppose that it would be in line with the unborn people's consent to be treated. The problem is that future generations may pay unforeseeable consequences arising from such a gene manipulation. However, also other routine therapies (such as chemotherapy) may cause detrimental consequences for those individuals that will be generated in a future by patients (deleterious genetic mutations transmitted to progeny). Thus, to me it is difficult to accept the intergenerational responsibility argument as a reason to oppose HIGM a priori and not to use this line of reasoning for other therapeutic medical practices. We can imagine a number of applications of the intergenerational responsibility argument that would lead to paradoxical conclusions (for example abortion ${ }^{4}$ ). Or we can use this argument to oppose other applications of human genetics (for example micromanipulation techniques used to compensate mitochondria genetic diseases) that have already been used to treat patients in the past (Donald et al. 1995 (4):316-319, Cohen (1997), Rubenstein (1995) or new technologies (introduction of artificial chromosomes to engineer human embryos) that may be used in the future without causing any gene transfer intervention (AAAS 2000, p.12). As such, I do not consider the intergenerational responsibility as an argument to oppose HIGM, but I do agree that we should take into account such a responsibility in decisions associated to HIGM.

The defence of human genetic integrity ( $H G l)$. 


\begin{abstract}
This argument posits the need to respect the human genetic lineage ${ }^{5}$. According to HGI, an inheritable germ line gene modification of an individual would transform the genetic lineage of man and, as such, it would be an unjustified intrusion of human beings in Nature. Can we claim that HIGM should be banned because HGl? First, it has been noted that "the human species does not have a "germ line" in the genealogical sense" (Frankel \& Chapman 2000). Then, the assertion that we should respect the human genetic lineage is controversial since the human genome is a heuristic abstraction (metaphor) to symbolise the intergenerational transmission of sets of specific genes. As we have seen in chapter 1.4 of this dissertation, classification criteria used in biology (biological taxonomies) do not cluster living beings through species-specific gene models since such models do not exist in Nature. Then, it would be conceptually debatable and biologically nonsensical to assert that we should respect the human genetic lineage if such a lineage is a human metaphor.

However, even if we assert that we do no have any right to modify the genetic lineage of a species, then we should avoid manipulating the genetic lineage of other living beings as well, from transgenic animals to transgenic crops. If we do not explain why 'human' genetic integrity should be protected and the genetic integrity of other animal species should not we defend an anthropocentric view that needs further justifications. We may say that HGI has a fundamental value in itself because man has an ontological status in itself, an intrinsic value so to speak (Sgreccia 1986).

This claim is extremely controversial and does not reach a consensus among ethicists at all (Mori 1998, Salvi 1998, 2000, Singer 1993, Parfit 1984). In my opinion, it is hard to claim that human beings have an intrinsic value 'because of' a disembodied element (further element) and subsequently link this factor to the human genome. If we do so, we implicitly claim that the human genome contains that further element. We then should assert that the ontological source of man is a specific gene or the human genome as a whole. Should we look for the gene containing the ontological value of human beings? The impossibility to identify that 'ontological gene' should induce us to oppose human genetics by claiming the intangibility of the human gene pool. But we do not have any reason to accept this view. Both the biological and the argumentative inconsistency of this claim induce me to consider the human genetic integrity argument as line of reasoning that cannot provide any valid argument to oppose HIGM.
\end{abstract}

Gene manipulation and Human Dignity (HD). 
Another possible argument against HIGM could be the claim that 'human beings have the right to receive a not-manipulated genome' because the gene pool represents the 'common heritage' of our species. A corollary argument says that HIGM is "a practice that could be contrary to human dignity" (Article 23 of the United Nations Universal Declaration on the Human Genome and Human Rights (1997). The Bioethics Convention of the Council of Europe or HUGO-ELSI statements on Cloning claim similar conclusions (see Fluss 1998). According to this line of reasoning, the main reason why HIGM would affect human rights is its potential interference with human dignity.

I have already stressed the inconsistency of this position elsewhere (Salvi 1998). The concept of human dignity is extremely controversial and hardly applicable to human genetics. In a sense that if we do not explain (a) what the human dignity is and (b) why, for example, a gene modification of a lethal monogenic disease would affect $H D$ we would be unable to say whether HIGM should be prohibited 'because of' HD. What is meant by the term human dignity? We may interpret HD in three different ways: (a) HD as an indicator of the socio-economic welfare of a given society; (b) HD as an ontological source of man; (3) HD as a biological feature of man. The first argument does not fit into a discussion about ethics and HIGM since it refers to socio-economic conditions of people living in given societies (it is particularly relevant to discuss issues related to justice or resources allocations or issues related to the third world countries). The second and third arguments can be rejected through the critiques that I have already posited to the human genetic integrity argument (an extensive discussion of this issue is in Salvi 1998).

If HIGM were not carried out to create 'monsters', or it would not induce suffering in the genetically modified individuals, why human dignity would be affected by HIGM? I do not see any reason to accept the human dignity concept to discuss the ethics of human genetics, and, to my knowledge, no arguments (empirical data, biological analyses and philosophical arguments) have been provided to explain the link between HD and the gene manipulation of human germ cells. Human dignity is a speculative concept, a corollary argument of the Declaration of Human Rights ${ }^{7}$. As such it can hardly fit into a discussion about ethics and the gene modification of future born individuals.

The impossibility to foresee the consequence of HIGM 
As we have seen in the previous chapter, bio-medical sciences tell us that to date we currently cannot foresee consequences associated to HIGM. However, the unforeseeable risks argument can lead to a prudential approach towards HIGM (safety, effectiveness, risks assessment) and it does not necessarily imply a moral argument against HIGM. A precautionary approach to HIGM is justified because of the uncertainties of this technology. The safety issues of HIGM are controversial due to both the state-of-the-art of human genetics (control of retroviruses) and the nature of such a gene modification (intergenerational transmission of genetically modified genes). In the previous chapter we have seen that subsequent mutations may occur causing the risk of consolidating genetic traits that do not have a positive outcome towards future generations (pathological mutations, epidemics, risks of iatromic arms, for example). Nevertheless, the risk of deleterious mutations that may occur in a future is inherent to sexual reproduction mechanisms as well. I do agree that HIGM involves unforeseeable risks, but this argument was never used to oppose other genetic modification of organisms' germ cells (such as somatic gene therapy). As such, I do not think that the HIGM ethical legitimacy can refer (only) to safety issues. Although these issues have a main importance in decisions associated to HIGM indeed.

The possibility to reinforce social and economical inequalities

We know that HIGM might promote a biotechnocratic power of civilised western countries to determine the biological nature of third-world people who do not have technological and economic possibilities to do so (Anderson 1984:401-409; Wood-Haper 1994:121 - 144). This power of economically wealthy societies is clearly an unfair scenario, because it would promote inequality between individuals, or developed and undeveloped countries (Wood-Haper, 1994:132). Nevertheless, HIGM per se does not involve its use in third-world countries as well as the production of socially cast off or exploited people (slaves). HIGM might be performed in both developing and Western countries. The use of HIGM to reinforce social and economical inequalities is a subsequent problem to the one of its ethical legitimacy. Protocols on good clinical practice may guarantee a fair use of such a technique around the world.

The problem of eugenics

As we have seen in the previous chapter, the claim that HIGM may be used as an intervention to fight a number of diseases that are currently 
causing the death of million people is controversial. According to biology, we do not know whether a potential disease will protect people from other pathologies (such as Malaria). We do not know whether a gene's defectiveness can be a transient quality or not (Suzuki \& Knudston 1988). But, we do know that the majority of inherited human traits tend to be polygenic and gene frequencies of allelic transmissible diseases cannot be decreased trough HIGM. According to this, the effectiveness of eugenic policies based on HIGM is scientifically problematic since both sexual reproduction mechanisms and selective pressures clearly obstruct this goal (see chapter II.2).

The argument claiming that HIGM may be used to enhance human characteristics beyond what is necessary to restore good health does not pertain only to HIGM but also to some applications of somatic gene therapy (see Juengst 1998:29-47). The eugenic problem is profoundly linked to HIGM. As I have already said, the human inheritable germ line gene modification could be seen as a tool to enhance human beings for pseudotherapeutic purposes (therapy versus enhancement) or to perform a largescale public health policy for lethal diseases that currently cause the death of million people in the world. Both these claims are biologically unrealistic.

As I have said, the debate over HIGM is still open. One major issue concerns the moral status of both the human embryo and the human genome. We have a strong tendency to attribute an intrinsic value to the human genome on its own ${ }^{8}$. Yet simultaneously, we have a strong tendency to emphasise the (potential) therapeutic value of HIGM (Salvi 1997). If we claim that embryos have a moral status in themselves, then HIGM would be ethically wrong because we have the duty to protect the (ontological) integrity of an individual during embryogenesis. On the contrary, if we deny the ontological status of embryos the heart of a discussion about the ethical legitimacy of HIGM would move from an ontological to a consequential approach. As I have already said we have reasons to use a consequential approach in the fields of human genetics since a number of philosophers have proofed that we have no reason to claim the intrinsic value of human embryos (Mori 1998, Singer 1984, Parfit 1984). In this dissertation I have continuously claimed that we cannot approach the problem of individuality through both ontological and dualistic explanations, since these approaches do not have any scientific validity and are philosophically debatable (see section one of this dissertation). As such I will explore HIGM through a consequential approach. 


\section{2. Pre-embryo's identity.}

When we stress the need to study the consequences of HIGM for the individual persistence over time ${ }^{9}$, we first have to respond to a specific question: 'Is a pre-embryo a biological individuality?' Prof. Alex Mauron, emphasised the ambiguity of the individuality-concept in biology:

\footnotetext{
"The zygote-as-individual definition, is probably the most convenient one for purposes internal to parts of zoology and to human biology (...). It is a genetic-based definition that takes genetic distinctiveness as a necessary (but not sufficient) constituent of individuality. (Mauron 1996, p.70)
}

I completely agree with this claim. As I have often said in this dissertation, we have no reason to accept the gene-eye perspective (see chapter 1-2). But this does not involve that genetic identity is not 'important'. If we think of the individual-concept in biology the role of genes towards the biological existence of living beings becomes central. The open question is: 'When we talk about human genetic identity, are we talking about the individual's chromosomes, or genes, or genetic structure?'

The claim that gene identity is the individual's chromosomal structure is controversial. The definition of human life as the existence of at organism possessing 46 chromosomes does not explain the nature of triploid zygotes (1-3\% of human birth), or a chromosomal anomaly (Klinefer syndrome, Down syndrome, Turner syndrome). Do we have to consider, for example, Down's syndrome patients as 'non-human' living beings? The second definition (human genetic identity as a specific set of genes) is denied by gene polyformism. The third one (human genetic identity as a genetic structure) does not explain how we can identity the individual's genetic identity since we do not have a complete human genome map. In addition to this, the gene structure of an individual is a plastic and variable dimension. The possibility that during embryogenesis the zygote duplicates in two monozygotic twins makes the genetic-identity argument really complex. Other biological phenomena that make problematic the equation " 1 oocyte + 1 spermatozoon $=1$ embryo $\rightarrow 1$ individual' are:

1) $80 \%$ of the fecundated oocytes dies because of natural pre-clinical abortions; 2 ) Zygotes cun evolve in carcinoma or tumour; 3) After the implantation the 25-30\% of pre-embryo's cells will form the placenta, embryonic membrane, and umbilical cord These cells and tissues will be successively destroyed; 4) The somatic cells' $X$ 
chromosome of female human beings will be inactivated only after the 14-16 day of Embryogenesis (Fiamminghi 1997:22).

These biological features of an organism weaken the genetic identity argument. In the light of this, Mauron's emphasis of the convenience of this notion in biology and bioethics is correct. However, it is my opinion that we can defend a narrow version of the genetic identity argument based on the 'role' carried out by DNA during embryogenesis. I will claim that genetic identity is important because: 1) it has a primary role towards the biological development of individuals; 2 ) it is not the main feature through which an individual's existence can be explained, it is one of the basic constituents of his/her biological individuality; 3 ) we have to think of individual's existence as a plastic process.

Let us come back to our initial question: is the pre-embryo an identity? In a biological point of view we know that: 1) pre-embryo is a class of similar elements (blastocysts are clones of the fertilised egg cell);2) the relationship tying pre-embryo's x-constituents is causal (chapter I.2);3) the DNA links causally embryo cells during embryogenesis. Embryonic cells are mutually interrelated, they are clones of the fertilised oocyte, and they will be causally related with future cells generated by their continuous duplication. This factor assures the causal relatedness and maximumsimilarity condition among constituents of an organism (Salvi 1997:53-77). Embryonic cells are identical (totipotent) and they have a teleonomic function: to form a new organism. Depending on this programme, regulated by the nucleus genetic information, an embryo develops. Thus, pre-embryo constituents are not simply cells tied by chemical relations, or a space relation, but they form an evolving unique entity. The genome unifies the pre-embryo's blastocysts in a unitary 'organism' which is persisting over time. Both the unitary meaning of pre-embryos and their capacity to behave as a whole make pre-embryos biological individualities ${ }^{10}$ (chapter I.1-I.2).

\subsection{Germline Therapy and the Individual's Identity.}

As 1 have said, the moral status of an embryo and the consequences of its transformations constitute a central point of the debate over HIGM (Madden 1998). Yet philosophers are discussing how to define the moral status of a human embryo. A consensus on this topic does not yet exist. For example, utilitarians consider the transformation of human germ cells ethically irrelevant because it is performed during a phase of embryogenesis 
where the central nervous system is not yet formed (totipotent cells are not differentiated). According to utilitarians, we have no reasons to assert that such a gene transformation would change something 'for' an embryo since it develops in any case. The main claim of this position is that people are 'individuals' because they are self-aware entities. According to utilitarianism, a gene manipulation of embryonic cells does not influence the persistence of a subject since at the moment of the gene manipulation he/she does not have the minimal condition to be considered an individual. Clearly, this idea refers to a personhood conception that attributes a moral significance to self-aware entities ${ }^{11}$ (Singer 1990).

However, even if an embryo does not 'feel' the transformation, it still undergoes one (Salvi 1997:53-77). The phenotype expression of the 'transformed DNA', makes the subject something other than what he/she 'should have been'. This element should be taken into account in a global moral analysis of HIGM, in a sense that if we want to transform the gene identity of a subject we have to consider this transformation as a factor to take into account in decisions associated to HIGM. As I have already said, a number of bioethicists have emphasised the embryo's capacity to 'feel' as a pre-requisite to be an individual. Nevertheless, this clain seems to me ambiguous. On the one hand, we have no reason to posit that an embryo becomes an 'individual' when he can experience. (It has been noted that human brain is completely functioning only after two years of life -Kurilo 1996:8.) On the other hand, embryogenesis is a process in flux. Thus, it is hard to fix an embryo's (biologic) feature having a value so strong to give him/her the status of individual. The context-dependence of this point of references is evident when we realise that our ideas on 'what is important to be humans' derive from cultural as well as societal factors. Since identity is a synergetic and unitary process, in which a number of determinants coparticipate in the existence-persistence of an individual (chapter I.1, 1.2, I.7), we have no reason to restrict what it means to be a human to self-awareness (see chapter 1.6 of this dissertation). Then we have no reason to attribute a basic value to any specific biological features of a subject, from his/her genome to his/her brain, from consciousness to behaviour. On the contrary, we ought to emphasise the complexity of organisms' existence. The absence of the central nervous system is a biological feature of pre-embryos. At this stage of embryogenesis, organisms develop because of information encoded in their genetic makeup, then the transformation of this element is highly problematic, even if the primitive streak of an embryo is not formed and nervous system elements do not appear yet (McLaren 1989:6249). 
In the previous chapters of this dissertation we have seen that, according to the organic unity theory, an organism is a unitary entity composed by heterogeneous constituents correlated by each others to (a) adapt to the environment (homeostatic functions), (b) act as a unity, (c) persist over time as a diachronic individuality. We also have seen that this mechanism refers to a number of biological-psychological processes (clustering processes) based on two dynamic processes: (a) the causal correlations among an organism's constituents (at different levels of complexity), (b) the maximum similarity condition. Both mechanisms regulate the biological existence of an organism at a determinate time and through time. This means that the organic unity theory does not claim any ontological notion of individuality but posits a model of the development of life based on biology. In those stages of an organism's Life where some biological factor has a much more important value compared to others. For example, during the blastocyts stage of embryogenesis, the role of DNA is basic. The modification of such an element does involve profound implications towards both the existence and the persistence of the developing organism. This is not an ontological or metaphysical statement, but a statement based on biological observations. When we modify an embryo's DNA at the totipotent stages we do affect its biological continuity.

\subsection{The Persistence of genetically modified embryos and the Closest Continuer Scheme}

Let us consider an alternative view that might help the ongoing debate on HIGM. If we approach an embryo's persistence over time, we see that HIGM causes a bifurcation in the continuity of a subject. Let us apply the Closest Continuity schema (Nozick 1981) to pre-embryo continuity. According to Nozick:

$X$ at $\mathrm{T} 2$ is the Closest Continuer ${ }^{12}$ of $\mathrm{P}$ at $\mathrm{t} 1$-without DNA transformation-, and $Z$ is the Closest Continuer of $P$ at $t 2$-after the HIGM-. If $Z=X$ then HIGM does not affect the continuity of $X$ 's identity.

If we follow Nozick's theory, $X$ (at $t 2$ ) continues $P$ (at $t 1$ ) because it is its successive development phase. Constituents of $X$ (the cells) continue in $X$, and in the same time the unity $P$ continues in $X$, because it satisfies the 
conditions of a causal relationship to P's properties. If P's properties are modified, the relation of identity continuity falls. This is because genetic transformation reflects to both Z's constituents and Z's identity. The expression of a "new" genome than starts (the ectopic translation of the genetically modified character), which is not tied by a causal relation with $\mathrm{P}$ 's requirements. $\mathrm{Z}$ is not $\mathrm{X}$ because it bares different properties than those typical of X. P has only one closest continuer: $X$ at $t 2$. The phenotypic expression of an organism's genetic identity is deeply affected by HIGM and this causes the 'end' of X. HIGM breaks the continuity of embryogenesis. P at T2 is not the same entity as $Z$ at $t 2$. Z's identity will continue with its closest continuers, but it is not the evolution of $P$. This means that modifying the genetic structure of a subject infers damaging the identity persistence of an organism during embryogenesis. Human inheritable germ line gene modification breaks the natural progression of embryo development because it interacts with both the biological features and the logic of individuality of the subject that undergoes it. HIGM does not change only the 'organism' (genetically modified germ cells differentiate in tissues), but also one of his/her main factors of unification: the phenotype translation of a genotype.

The identity of X is also modified if X's genome is not considered the most important element of X's identity (gene-centric perspective), but one of his fundamental requirements. This is because $X$ results 'also' from information encoded in $X$ 's genome. A gene manipulation of embryonic cells will influence the complete mechanism of gene expression. The bio-engineered organism $(Z)$ will be an individual that is different from $X$, and he/she will transmit his/her manipulated genotype over time (progeny).

\subsection{Conclusions.}

We have seen that HIGM is problematic because it involves a number of ethical factors (intergenerational responsibility, human dignity, and so on) that should be taken into account in research involving human subjects. However, we have also seen that none of the arguments above mentioned can provide strong reasons to oppose such a technology. Because of this paradox, I have focused this chapter on identity problems arising from HIGM. According to both the organic unity theory and the closest continuer scheme, HIGM interacts with the persistence of an individual as a determined entity. In fact, HIGM does not only change X's embryogenesis, but also it alters the causal relatedness and similarity-condition among X's 
constituents. (Since human genetics cannot control the planting of the bioengineered nucleus sequences, the causal role of the individual-specific genetic identity is substituted by a 'fortuitous' mechanism of gene modification.)

If, as I assumed, individuality is at the least 'important', then HIGM is a technique that has a negative connotation in itself. Because it does not respect the persistence of an individual $X$ over time and it creates another individual $\mathrm{Z}$ on the body of $\mathrm{X}$ who 'does not exist any more'. However, this initial negative connotation should be related to the positive consequence achievable via HIGM (for example a successful treatment). Since HOGM is highly problematic from an ethical point of view we do need concrete positive outcomes (for example a successful treatment) to claim the ethical legitimacy of HIGM. According to results achieved by somatic gene therapy and the sequence of the human genome (Science (291) 2001) we are far to use HIGM as a treatment. This is not only for technical reasons (such as difficulties associated to control vectors of exogene genes) but for heuristic reasons as well. In the post-genomics era, human genetics will focus on gene expression, proteomics and structural genomics. This means that the claim that HIGM would be a therapeutic practice is at the least premature. Then we should not carry out HIGM.

\section{NOTES}

I The claim that human inheritable germ line gene modification (HIGM) should be banned appears in several legal documents. See: Pluss, 1998.

${ }_{2}^{2}$ Stem cells and in utero gene therapy may be an indirect form to genetically modify human germ line (Annas et all. 1999, Bloom 2000, Caplan \& Wilson 2000). The debate about the cthical lcgitimacy of such applications of biotechnology is still open (Vogel 1999, Barbur 2000). The open questions are; "to what extent should the modificalion of human germ cells be permissible?' 'Why bioengineering human germ cells is a ethical problem?'

${ }^{3}$ In this chapter I will not address issues related to culturing ES and EG cells. Both these applications of human biotechnology have renewed discussions about the ethics of research involving human embryos and cadaveric fetal material. I will not explore issues related ES and EG research (see NABC report September 1999) because they do not concern issues related to the continuity of an individual's Life but other issues (such as the moral status of embryos at an early stage of embryogenesis) that do not differ from the ones of human germ line germ gene modification.

${ }^{4}$ The problem is that when wo talk of 'unborn' to discuss HIGM we refer to future generations rather than the patient treated via HIGM. Is the interest of future generations a factor so strong to annul the interest of the patient who undergoes a therapy? We may say that (1) the number of people who will undergo the consequences of HIGM growths cxponentially (via sexual 
reproduction) and then (b) the extensive long term consequences of HIGM should be considered as a key factor to take into account in decisions associated with HIGM (Salvi 1998). According to this argument, we could say that because of the high number of future born pcople growths exponentially we should avoid authorising HIGM. However, this claim may also be used for other issues related to reproduction, for example abortion. The number of potentially unborn people (non-generated by the non-born individual) may also be considered as a key factor to discuss the ethical legitimacy of premature termination of pregnancies. But this is not the case. Why should this factor be relevant for HIGM and not for other problem issues?

${ }^{5}$ The idea that human genome is a value dimension to respect and safeguard has been claimed by Werner (see Singer et al. 1990). The central argument of Werner was that pre-embryo's genome is the distinctive feature through which individuals are classifiable as Homo sapiens. This idea has induced Werner to use the genetic identity argument as a moral principle. In the light of the analogy principle, Werener said, when we think of zygotes as human beings, it follows that it is morally wrong to manipulate (gene manipulations, IVF), destroy (abortion), or use them (frozen embryos, spare embryos), since they are moral subjects. Indeed, in a biological perspective, this idea is controversial (Singer et al. 1990).

6 "The human gene pool is a heuristic abstraction, not a natural object, and lacks a material referent in naturc. Individuals inherit a specific set of genes derived from their parents. Thus from a biomedical perspective, there is no intergeneration "human germ line" that could serve as an asset to the future. While it is important to ensure that future generations have fair access to the benefits of human genetic research, it is conceptually mistaken to interpret the human gene pool as an "endowment" accumulated by the wise investments of natural selection over which we now have stewardship. The evolutionary process that controls the allelic content of the human gene pool is an unmanaged and unmanageable onc. The human gene pool is not a stable given, but has been in flux over the course of human history." (AAAS Report, September 2000)

${ }^{2}$ In the Presentation of the Preliminary Draft of a Universal Declaration on the Human Genome and Human Rights weread (p.6):"The principles of the protection of persons with respect to the consequences of rescarch on the human genome arc based on a set of rights which flow directly from the principle of dignity: The right to equal treatment (...) the right to individual freedom (...) the prisciple of solidarity among people and countries". These principles are contained in the Universal Declaration of Human Rights (10 December 1948) (DHR). This means human dignity is based on a legal system, which constitutes the moral basis of its acceptance. When IBC identified DHR as the constitutive source of human dignity, it accepted a body of "rightsvalues" as the fundamental co-ordinates of human society. According to this, human dignity is not the milestone of science, but is DHR to have this value. The acceptance of human dignity refers to the acceptance of DHR. Human dignity is only an extension of the "right to individual freedom". The fundamental steps, which conduce to human dignity as peculiarity of human beings, are contained in the first two articles of IBC's Declaration. In the first one we read: "The human genome is a fundamental component of the common heredity of humnnity", in the second one "The genome of each individual represents his or her specific genetic identity". Both these principles are based on legal declarations (Article 1: Convention on the Law of the Sca of 10 December 1982; Convention for the Protection of the World Cultural and Natural Heritage of 16 November 1972, The Declaration of the principles of International Cultural Cooperation of 4 November 1966, Declaration on Race and Racial Prejudice of 22 November 1978; Article 2: Universal Declaration of Human Rights of 10 December 1948). When we pass 
form "article 1 " to "article 2 " we see that human genome is considered as a "requirement" of human individuality. In this sense IBC defines two dimensions of value in human genome. The first one refers to the view considering genome as a "shared peculiarity" of humans; the second one considers genome an "individual "property". Transforming the individuals' genetic identity means interacting with something that is "possessed" by the single person as well as individuals constituting (over time) the "human race". (Salvi 1998)

'I talk about 'intrinsic value' because even if at a molecular level the species-boundary between human and non-human beings is quite null $-99 \%$ of the chemical information stored in human DNA is indistinguishable from that of an ape (Suzuky 1988:332), however the human gene pool is considered a 'dimension to sateguard'. This ethical difference between human and non-human DNA is defensible only in an anthropocentric and ontological perspective.

${ }^{9}$ We should be aware that, whatever notion of individual we use in approaching the moral legitimacy of HIGM, it is a fact that HIGM modifies the biological matrix of a genetically manipulated individual. The first consequence 'for' the modified organism is its genctic transformation. According to this, when we address issues related to HIGM we should, at the least, be able to explain why this technology should be rejected or accepted.

${ }^{10}$ I consider the genetic endowment as a causal element of organism's existence since DNA has a central role in embryogenesis, namely it safeguards the condition of maximum similarity and causal relatedness among embryo cells. Clearly, this role weakens in successive phases of organisms' Life, since other biological mechanisms will be more significant for the organism's individuality after embryogenesis. However, in absence of these mechanisms (I mean the homeostatic systems, neuro-systems, physiological; systems and so on), the genetic identity is important because of its role in the development of organisms. If this is not clear, my criticisms over biological reductionism would be nonsensical.

11 "Self-consciousness is not simple awareness, rather it is awareness of awareness. To value its own life, a being, would have to be aware of itsclf as an independent centre of consciousness, cxisting over time with a future that it was capable of envisaging and wishing to experience ...The capacity to value existence in this sense ...it does not require rationality in any very sophisticated sense of that term, merely the ability to want to experience the future, or to want not to experience it and the awareness of those wants" (Harris 1985:18)

12:The Closest Continuer view holds $y$ at $t$ " is the same person as $x$ at $t$ ' only if, $y$ 's propertics at $t^{\prime \prime}$ stem from, grow out of, are causally dependent on $x^{\prime} s$ properties at $t^{\prime}$ and, there is no other $z$ at $t$ "stands in a closer (or as close) relationship to $x$ at $t$ than $y$ at $t$ " does". Nozick, 1981 . p.36 


\section{I1.4. Transforming Animal Species: the Case of 'Oncomouse'}

During the 1970's, the first chimeric mice were produced. The rapid development of techniques used for the creation of transgenic animals permitted successful applications in mammals. In 1975, during the Asilomar Conference restrictive rules were formulated to forbid experiments in some fields of biotechnology, such as: human genome, transformations of pathogenic organisms or carcinogenic organisms, transmission of drugresistant genes, and introduction into the environment of artificial organisms ${ }^{1}$ (Brenner 1978).

These guidelines have been followed in human genetics, but seem to have been ignored in animal biotechnology. Animal experiments are frequently used as research tools in biotechnological industries or laboratories. This implies that, even if philosophers and biologists have not yet clarified the moral status of animals, the transformation of animal and yegetal life forms is a routine technique used in animal breeding or research.

For example, multinational industries producing milk or meat often use biotechnology to increase their production (Bovine Somatotropine -BST) hormone increases by $20-40 \%$ the milk production for each animal - Rollin 1989; Fox 1992; Kaiser \& Wellin 1995). Biotechnology has been used as a tool to maximise animal breeding. Examples of such a use of animal biotechnology are, inter alia: beltseville pigs (King 1996, Rollin 1997), broiler chicken (Broom 1998, Raw et al. 1998), double-muscled cattle (Broom 1998, Sandoe 1999).

This technology has been used in pharmacological industry as well: pharmaceutical production of proteins, hormones, bioreactors (Bosom, 1989:263), and so on. Bio-engineered organisms, are 'produced' every day all across the globe, even if the moral implications of this action are not yet understood.

\subsection{Transgenic Animals}

The term 'transgenic' describes organisms containing integrated copies of genes or gene constructs, derived from other species, or not 
normally found in animals in their chromosomes. These transgenes may or may not be expressed. Transgenic animals can be generated by introducing the cloned DNA of foreign genes into fertilised oocytes or by microinjection into the pronucleus (or other techniques). These oocytes are subsequently transferred into the uterus of a pseudo-pregnant recipient animal where they develop. At this moment, transgenic animals are frequently mice, but major research efforts in transgenic animal modification are now focusing on calve, pigs, rabbits, sheep, goats ${ }^{2}$, and fish $^{3}$. Current approaches to generate transgenic animals fall into two broad categories: direct manipulation of the zygote or early embryo; and manipulation of pluripotent cells in vitro. Even though these are the dominant techniques, new methods are currently being sought 4 .

Techniques utilised to achieve this aim are numberless. The first one is the introduction of foreign DNA into a fertilised oocyte. The second one is the introduction of transformed cells of foreign DNA in embryos. The third approach is the transformation of somatic cells. Other techniques used for the creation of transgenic animals are the embryonic stem cell-mediated gene transfer, ${ }^{5}$, retroviruses, the splitting of pre-embryos that can be developed in several clones ${ }^{6}$, and the nuclear transfer ${ }^{7}$.

Biologists produce transgenic animals to different purposes, one of which is to study human diseases. In fact, cell cultures cannot give information about all cell activities. Biologists can study these activities via bioreactors (transgenic animals) and analyse factors causing gene activity (or inactivity) that can be expressed only in embryos or in staminal cells. According to this, biologists observe the expression of endogen genes in transgenic animals. In fact, oncogenes or other pathogenic agents ${ }^{8}$ are introduced in an animal's genome (let us think about Lesch-Nyhan Syndrome, or hypertiglyceridemia -Ito, 1990: 790-793, Kuhen 1987 -apolyprotein CIII-). Recently, biologists began using transgenic-mice to study the expression of Aids in the human immune deficiency system. Other animal applications for medical purposes have been: 'severe common immune deficiency (SCID)' tetracarciroma, and Alzheimer (King 1996, Thompson 1997).

The basic aim of the above-mentioned applications is to 'construct' a living model (bioreactor) where it is possible to observe cellular mechanisms and gene expressions of pathological factors. In this view, animals are considered as 'experimental tools,' which are more interesting than cell cultures (let us think of experiments done to study -fetoprotein or immunoglobulines). Other animal applications of genetic transformations 
have been done in order to achieve other goals. For example, the Human Growth Hormone has been planted in different animal species: fish (Gill, 1985:643-646), rabbits and pigs, cows (MacKenzie, 1990). These manipulations allowed for different applications of biotechnology in animal breeding ${ }^{9}$.

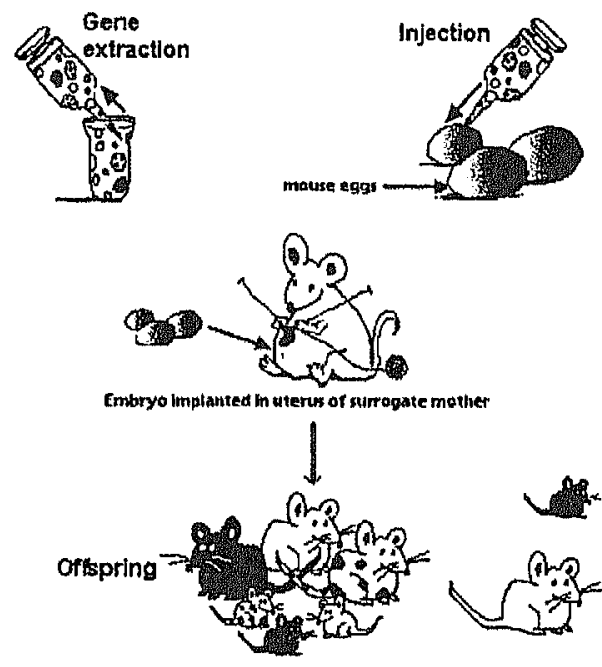

Tramegenic Mico

We can schematise the purposes for which transgenic animals have been used in biotechnology as follows: (1) medical research (to study specific factors in complex homeostatic systems via expression of modified genes); (2) toxicology (detection of toxicants); (3) mammalian developmental genetics; (4) molecular biology (study of gene expressions); (5) pharmaceutical industry (target production, drug production and pharmaceutical proteins); (6) biotechnology (production of specific proteins, genetically engineered hormones or animal breeding applications, xenografting) 


\subsection{Problematic implications of transgenic animals production.}

The first risk of transgenic animals production is the possibility of influencing ecosystem balances by introducing them into the environment. Transgenic animals may compete with local animal species, eliminate their natural enemies (Boer et al. 1995, Rollin 1996), or simply modify the nutritional flux of a biosystem (alimentary chain -Terragni 1987:99-106). Depending on the geographical conditions of ecosystems, the consequences of this introduction may vary. Isolated and fragile ecosystems (islands for example) would pay higher consequences. Continental ecosystems may also be influenced by cohabitation of bio-engineered and natural species. We cannot predict the consequences of GMOs into the environment. The microorganic ecosystem is a complex and rich natural world. As we still have only a superficial knowledge of microbiotic balances in Nature, it is difficult to predict what consequence introduction of GMOs into the environment may provoke. Similar remarks can be claimed for the introduction of transgenic animal in Natural landscape (Sandoe \& Holtug 1993).

In parallel, we cannot foresee the consequences of this diffusion towards evolutionary mechanisms (genetic or phenotypic evolution). We therefore have no idea what the ecological and biological consequences of introducing transgenic animals into the biosphere may be (Hahn 1996, Rollin 1997). A list of possible consequences includes:

- Changes of genetic information may lead the transfer of GM gene from one species to others;

- Consequences towards microbiotic balances;

- Possibility of having subsequent mutations causing new pathologies (epidemics).

The debate over the ecological risks of animal biotechnology continues. Some biologists underlined that until we have a better understanding of genetic exchanges between different species in Nature, we ought to respect 'inter-species boundaries' (Suzuki \& Knudston 1988), but other scientists say that continuous horizontal gene inter-species communication by eucharyotes exists in Nature (or the 'conjugation' process in prokaryotes), and that plants have continuous changes of genetic information among species that do not cause relevant consequences for ecosystems.

We may say that since we do not (and cannot) have data concerning the ecological (or evolutionary) consequences arising from the delivery of TAs 
into the environment (Sandoe 1999:44), then we should avoid using this argument to oppose animal biotechnology. The problem is that we cannot have this data! Do we have to take this risk? Let us consider this problem an open question. Here I will deal with a specific problem: what ethical implications does the production of transgenic animals arise? I will investigate this problem by analysing a specific problem case: the 'oncomouse ${ }^{10}$. Motivating factors encouraging this analysis are: 1) It represents a well-known case for scientists; 2) It is a biological patent legally recognised; 3 ) Its creation responds to a practical need of science: the study of oncogene; 4) Its transformation determines the pathology in the mouse (the creation has the aim to show this pathology).

\subsection{Why choose the example of 'oncomouse'?}

The 'oncomouse' was the first animal patent produced by biologists ${ }^{11}$ (Hubbard \& Krimsky, 1988:6-7) of Harvard University (patent n. 4.763.866, for Non-human Transgenic Mammals, 1984). The technique used to transform the mouse's DNA was to infect embryos with retroviruses. The aim of this manipulation was to investigate the expression of a human tumour causing limb deformity in transgenic mice co-segregated with MMTV-myc at the integration site. ${ }^{12}$

Biologists planted a genetically engineered exogenous DNA into mice. The inserted mutation caused a severe defect in the pattern of limb formation in the developing mouse. The novel recessive mutation was phenotipically identical and non-complementary to two previously encountered limb deformities caused by a dominant mutation (limb dysphormism). The inserted element thus provided a molecular genetic link with the control of pattern formation in the mammalian embryo. In order to ensure the transmission of the DNA transformation, and to enhance the chance of survival, the affected animals had to be removed from the competition of their wild-type littermates during nursing. Several oncogene constructs were introduced into mice to elicit tumorigenic or other pathological changes. The c-myc genes were fused to LTR of MMTV. Transgenic females, in one line, characteristically developed mammary carcinomas during their second or third pregnancy (Stewart \& Pattagrale 1984). In another line, mice developed testicular and lymphocyte tumours. The MMTV-myc integration in the DNA caused limb defects. The abnormal regions were: a) Synostis of 
the radius and ulna; b) Synostis of tibia and fibula; c) Fusion of some tarsal and metatarsals and of the digits of fore paw, as well as fusion of some carpal and metacarpals (Palmiter 1986:465-499).

The examination of genetic maps detailing mice chromosome 2 revealed the presence of mutations that affected the development of the fore and limbs. The most important mutation was the limb deformity (ID). The produced lines of mice transmitted the integrated nucleus sequence in the next generations. The manipulation of mice's germ cells allowed the expressions of neoplasms in the entire animal. A new race of mice having human neoplasms was 'created': micro-laboratories to study human cancers.

\subsection{The Moral Implications of the oncomouse's Production.}

If we aim to explore the moral implications of this bio-technique we need to consider: 1) the consequence of this transformation for the GM animal; 2) the expected benefit of this action; 3 ) the moral status of mice; 4) the possibility of alternatives; 5) the tension between (1) - (3). When we apply this scheme to oncomouse, we see that we have clear information related to the first two elements of my scheme: we know that the oncomouse gets a human neoplasm (1) and we perform this gene manipulation because we aim to study the cell expression of oncogene in the phenotype (2). We may say that both these elements allow us to say whether the technique is scientifically effective. Nevertheless, if we do not consider the consequences 'for' the animal as significant elements to take into account regardless of the achieved scientific goals, we automatically deny the moral status of animals by following an anthropocentric approach ${ }^{13}$ (from Aristotle to Kant, from Spinoza to Descartes). The anthropocentric claim (speciesism -Singer 1990:6) refers to Kantian philosophy thinking of rationality as a pre-requisite to be 'moral agents'. Currently this position has weakened because the claim that animals do not have a consciousness does not reach a consensus among biologists at all. Experiments on the capacity of animals to grasp and utilise languages (Griffin 1976; Gallup 1982:237248 ), or animal behaviours (den Boss 1999), or animal 'sentience', show that we have numerous evidences to consider animals as conscious individuals (Rachels 1990, Rollin 1989). Even if the human consciousness expresses by rationality and language, this does not mean that the absence of linguistic and rational capacities (based on human standards) in other animals implies that they are incapable of having a consciousness ${ }^{14}$ (see the 
distinction between primary and secondary consciousness in I.9). Then we cannot claim that those entities that have different standards of consciousness (compared to the humans') should be declared 'unconscious' or 'pre-conscious' individuals ${ }^{15}$. Again, as I have already said, studies of animal behaviour show that 'consciousness' pertains to a number of animal species, and has species-specific features (Griffin 1976, 1984; Walker 1983, Rollin 1989). Animal consciousness is different from humans' (van den Bos et al. 1994).

The question arising is the following: 'If animals are sentient entities, why do we place a moral difference between human and nonhuman beings?' This is a controversial topic. Philosophers defended the moral status of animals through a number of theories: An animal is a moral subject because it is: (a) able to feel pleasure and pain (Singer 1990, 1993), (b) subject-of-a-life (Regan 1982,1983), an element of biodiversity (Norton 1987, Sandoe \& Holtug 1993).

The first theory (a) refers to the philosophy of Bentham and Mill and has been advocated by Peter Singer. This Australian philosopher considered an animal capability to suffer as a sufficient condition to be included in the moral community. According to Singer, actions causing pain in sentient animals are morally unacceptable. The conceptual basis of this claim referred to utilitarianism. According to this philosophical theory, in those cases in which we cause pain to a sentient living being, we carry out an action that is ethically wrong. This means that animals are moral actors and we cannot use them for human purposes. This claim has been criticised by a number of philosophers (Rollin 1989, Fox 1992, Fincham \& Ravez 1991, Kaiser \& Wellin 1995). Here I will stress the limits of this view if used to explore problem implications arising from the production of transgenic animals.

If we think of the oncomouse through utilitarianism lenses, we should condemn this technique. The animal gets a human pathology resulting from a gene manipulation and causing the animal to suffer. However, suppose that we transform oncomouse's genotype by inserting a gene that, when expressed, will obstruct its mechanisms of neurotransmission (the transgenic mouse would not have cerebral areas of pain). In this case, we would not cause an increased suffering in the animal. Utilitarians should accept this use of animal biotechnology. The oncomouse would not 'feel' the human cancer, and this action would be morally legitimate since oncomouse is thereby not a moral entity anymore, having it lost its sentience-capacity. Should we conclude that those genetic manipulations 
that cause so serious physiological dysfunctions to interrupt the GMO's sentiency capacity are morally right? According to the animal-suffering criterion we should accept this conclusion but this would be contradictory to the defence of animal rights that is based on Singer's philosophy ${ }^{16}$.

In parallel, if we accept utilitarianism, we may overlap human interests to animal interests. Conflict situations, in which the interests of two moral entities are involved, should induce us to privilege the interests of that moral entity which has a higher moral value in itself. When we have to choose between 'killing a human or an animal' for survival, we decide to kill an animal even if it is a moral entity. For example, in 1999 soldiers in southern Malaysia killed more than 300.000 pigs to stop an epidemic of encephalitis epidemic. This means that we can always define a principle of utility that justifies the use of animals for human purposes. Again, this claim is unacceptable for advocates of utilitarianism.

According to the criticisms expressed above, I do not consider utilitarianism as a theory that can help us in approaching issues related to animal biotechnology. Utilitarianism does not offer the possibility to defend animal rights in cases of conflict with human interests.

If utilitarianism cannot help us in this research we have to look for other theories on animal rights. Regan (1983) defended an alternative theory. He posited that animals have a moral value, because they are 'subjects-of-a-life'. This means that animals possess an intrinsic value because they are self-aware subjects. A number of philosophers have asserted that animals have an intrinsic value (Dol et al. 1999). They claimed that when we interfere with animal germ cells we transform the ontological source of a species, its genetic integrity (Verhoog 1999, Brom 1999). The defence of this principle refers to the principle of analogy according to which, stating that as we attribute a moral value to human beings, we ought to extend this ethical basis to animal species. However, this approach needs further explanations. Depending on the intrinsic value theory, shall we say that the moral status of humans and the one of animals coincide? When we equate the moral value of human beings with the one of non-humans, countless paradoxes reveal themselves. For example, we might attribute a moral value to pathological bacteria. Then, medicine would become ethically wrong because, for example, antibiotics causes the death of 'moral entities' (bacteria). This conclusion is clearly unreasonable. This means that the intrinsic value theory should define a moral difference among different animal species. Advocates of the intrinsic value theory as a value that pertains to all organic systems (Naess 1984) would contest the existence of 
a moral taxonomy in Nature. But this claim refers to ontological (axiomatic) bases and does not have any rational explanations. Natural sciences use biological taxonomies based on the biological features of organisms (se chapter I.3). How can we deny these taxonomies in approaching issues related to interspecies justice?

My second objection to Regan's philosophy is the following; How does a biotechnological transformation of a mouse interact with this further (disembodied) element? In those cases in which the inserted exogenous gene will be pathologically expressed, we can say that this action is wrong because we end the life of a subject-of-a-life. Nevertheless, if we only transform the animal's genetic make up, how can we define the moral implications of this action? In the case of the oncomouse, we can say that it is ethically wrong to induce this pathology to mice because we cause their suffering when the tumour expresses in the phenotype. However, if this transformation does not cause such a suffering (for example the 'supermouse' or the 'hairmouse'), how do we interact with their ontological nature?

We may also object to Regan that when we produce transgenic animals we cause the birth of living beings, thus creating subjects-of-a-life. In this perspective, the artificial 'production of animals is ethically positive. But, again, Regan's philosophy does not allow us to say whether such a use of biotechnology is ethically wrong.

All of these reasons encourage me to refute the ontological argument since it does not help us to understand what a non-pathological gene modification would mean according to the ontological status of animals, and it involves bjocentric ethics.

\subsection{Ethical implications arising from the production of the Oncomouse.}

Let us check whether we can explore the problem implications of TA production via a consequential criterion. The oncomouse's pre-embryo is a whole of cells spatio-temporally bounded. Data for its embryonic development are encoded in its genes. In this sense, when we modify mouse's DNA, we interact with its entire embryogenesis. Then our manipulation is problematic. If we consider a living being's genetic identity as something that should be taken into account (as we do in the case of human beings) then we should condemn animal biotechnology because it interacts with the oncomouse's existence. This initial conclusion needs to be 
referred to the expected goals of such a gene modification. This is because, as I have already said, the moral implications of an action are intrinsically linked to the consequences arising from it, while a theory referring only on this consequential approach does not solve all dilemmas surrounding the use of biotechnology (see my criticisms on utilitarianism). (When I kill a lion to ensure my own survival, it is a different act than the murder of an animal for a perverse pleasure.)

In the case of the oncomouse, the moral tension between consequences/finalities refers to a moral taxonomy, which attributes to human beings a moral predominance with regard to other animal species because human beings have a higher degree of biological complexity and they are moral community makers. According to my organic unity theory (see chapter 1.3 of this dissertation) and the assumption that living organisms have a minimal value in themselves as existing (chapter II.1), we know that a mouse has a moral value that strictly refers to its degree of unification, as an organic unity. Being the degree of organic complexily of a mouse less than the human, then the oncomouse has a moral status on its own but this initial moral parameter does not allow us to equate the moral status of mice with the one of humans. We have seen that the claim that animals have a moral value reaches a quite general consensus among bioethicists even if an extensive theory on this issue does not exist yet, we this can assume that animals have a minimal moral value in themselves. According to the scheme described in the previous pages of this chapter, the moral status of the genetically manipulated animal has to be related to the expected goals of such a gene manipulation. In the case of oncomousc, the finality of its 'creation' is to have a living cellular culture. It aims to increase the human knowledge of oncogene -in order to treat human cancer. This finality has a positive value, because it is oriented to an increase of medical knowledge. And the possibility of treating people is ethically positive according to different ethical views (because we may increase people's quality of Life). Is this outcome a sufficient ethical paraneter to compensate consequences paid by oncomouse; to express a human pathology? All the ethical criteria described before (animal welfare, animal sentience, animal rights to survive) may be used as arguments to define the ethical legitimacy of producing oncomice. But all of them would agree that the suffering of the genetically moditied animal is really hard to be 'compensated' by the positively of the outcomes (Anderson 1999, Boyce 2000:12-13). 
To me, the paradox of this case study, as well as of animal ethics, is that human beings have to decide whether actions they do for different purposes would affect the moral status of those entities that undergo such actions. If we are aware of this problem, we see that it is hard to defend an approach to animal biotechnology that would be both fair and reasonable. We cannot deny that human beings are the ones who define the moral implication of actions they do (moral systems makers). As such, it is hard to have an approach to animal biotechnology, which would be completely neutral and not a rational justification of the legitimacy of actions aimed to satisfy human needs.

In parallel, it is also hard to propose solutions that would defend a fair approach to animal issues (interspecies justice) because it is a matter of fact that human beings have continuously used Nature instrumentally. The destruction of natural landscape is a clear example of such an intrusion. The 'human' intrusion in Nature does not respect the animals' right 'to live in a natural landscape'. All conventional use of animals (for example animal breeding) may be seen as a violation of animal rights (Sandoe 1999). In this sense, I believe, those views equating the moral status of human beings with the animals' are unrealistic. This is also because we cannot think of the moral sphere of animals as based and built on "human' ethics. Human ethics is constructed on rationality and social rules, while animals have a different 'rationality' and social organisation ${ }^{17}$. How can we deny that, even if we open the moral community to animals, this claim results from a human decision (Blackburn 1984)'? How can we deny that humans would fix the pre-requisite for animals to be moral entities?

\subsection{Conclusions.}

In the case of the oncomouse if the germline transformation of the animal is the 'only' possible technique to increase the research on oncogene, I think, we cannot say that it is morally wrong. Even if the animal will express pathogenic factors resulting from its genetic manipulation. The finality of this action constitutes a relevant moral reference. This conclusion requires that no alternative methods exist to study neoplasms. If we can use alternative methods (e.g. use cell cultures, or computer simulations) to achieve the expected scientific goals, then we should avoid manufacturing transgenic animals. 
When we attribute a positive value to the heuristic increase of Science regardless of the implications arising from an use of new technologies, we think of biotechnology as something that is per se unproblematic. This would be true both for human and non-human biotechnology. Science, then, should respect both human and non-human beings because both are moral entities. Nevertheless, according to the moral prevalence of humans in a moral taxonomy, $l$ do not see any solution for TA than the possibility to justify (exceptionally) the creation of animals expressing (induced) human pathologies. I am aware that this conclusion is unfair, and can be accepted in exceptional circumstances where well-justified and clear benefits would arise from the production of a transgenic animal. Other solutions aimed at equating the moral value of animals with the one of humans, to me, claim unrealistic scenarios in which human beings should abstain to have an instrumental use of Nature or declare the intangibility of animals. None of the analysed theories advocating animal rights seems to produce a satisfactory argument to end animal biotechnology. Even the alternative solutions that have recently been claimed, such as the animal integrity criterion (Verhoog 1999) seem to help the current debate annong decisionmakers and experts of animal welfare. In this respect, the organic unity model allows us to introduce a moral taxonomy among animal species. Such taxonomy does not refer to metaphysics (or ontology) but to biology. As such it differs from other ethical theories on animal rights (from Regan to Verhoog) discussed in this chapter. In parallel, it provides an altemative view of inter-species ethics, as well as the possibility of evaluating the consequences arising from the manipulation of an organism's germ cells in regard with its continuity and identity. Then, even if I am not satisfied with this solution I do not see any other possibility than to allow the production of transgenic animals.

\section{NOTES}

\footnotetext{
1 The implications of recombinant DNA experinents analysed in the Asilomar Conference were; "Inserting genes for toxins into bacteria, Changing the host range of bacteria, Using animal viruses as vectors, Inserting genes coding for drug-rehated substances inlo bacteria, Cloning DNA from animal viruses, Randomly inserting DNA from higher organisms into tower organisms, Dispersing antibiotic-resistant plasmids in bacterial populations" (Suzuky \& Knudston 1987:229)
} 
2 Proceedings. of the National Academy of Sciences 91, 1994:6840-4, Bioleclmology, 12, 1994:699-702

"Windsor and Hutchinson (1990); Marine laboratory Aberdeen (1999).

${ }^{4}$ Tsukui 1996: 982-5, EIAIB News 1998, Nature Biotechnology, 14, 1996:942-3, Straughan $(1999), \mathrm{p}: 19$

"This method involves prior insertion of the desired DNA sequences by homologous recombination into an in vivo culture of embryonic stem cells. (CCAC 1997). This technique is particularly important for studying the genetic control of developmental processes and is particularly used in mice.

"Pre-Embryo cells are ctoned for producing normal cellular complements and for re-forming bio-engineered embryos (Soltor 1987:23-27). New approaches to animal transgenesis are discussed in Nature Biotechmology /4 (1996), 942-3; Tsukui, T. et al. "Transgenesis by adenovirus-mediated genc transfer into mouse zona-fiee eggs", Nature Biotechnology 14 $(1996), 982-5$.

${ }^{7}$ NTT technique is described in Nature (380) 1996:64-66

"Iluman disease models in transgenic mice jnclude: $\beta$ thalassatmia, renal pathology (Cell (83) 1995:473-82), motor neuron defects (Cell (83) 1995:313-22). carcinomas (Nature Medicine (1) 1995:792-7), heart and lung disease (Nature Medicine (1) 1995: 749-51) AIOS research models (TIBTECH (13) 1995:142-50), tuberculosis for vaccine developtnent (Trends in Microbiology (3) 1995 : 418-24).

${ }^{9}$ In 1986, for example, the discovery of the fertility gene increased industrial applications of transgenic animals in animal breeding (Sandoc 2000). The ovulation rate of merino shcep has been increased without conseguence towards the production of wool (Macer, 1990:123). Fish have been genetically manipulated for increasing their dimension and oocyle productions, (BBSRC: 1949:19).

${ }^{\text {in }}$ In this chapter I will not repent what I have already said about problem implications arising from such a manipulation of mice' germ cells towards identity-persistence problems. What 1 have said for human applications of bioengineering orgatisns' germ cells concerns also the continuity-problem of non-luumans.

"Since the "Oncomouse" is the lirst genetically modified organism that has been patented in the United States (patent n. 4.763.866, for Non-human Transgente Mammals -1987-), and successively patented by the European Patent Office (European Patent Specification, n. $85304490.7, \mathrm{C} 12 \mathrm{~N} 15 / 85$ (GOIN 33/574 A01K 67/027-1992-), the analysis of those conceptual (and legal) reasons that have induced the Luropean Patent Office and the United States Public 'Trademark's Office to consider the Oncomonse patentable, become central elements to analyse in the fields of biotechnology. In this sense, the analysis of the case of Oncomouse is an extraordinary example of how biologists, philosophers, and politicians bave reacted in possibilities of genctically modifying onganisms.

${ }_{12}$ In the European Patent Specification, n. 85304490.7, CI2N 15/85 GOIN 33/574 A0IK 67/(27 (European Paten Office), this patent is described as follow -p. 55-: "The presen invention now provides a melhod for producing a transgenic non-luman mammalian animal having an increased probability of devcloping neoplasms, saicl method comprising chronosally incorporating an activated oncogene sequences into the genome of an aneestor of said animal".

${ }_{13}$ According to anthropocentrism; a) Nanure exists only in ralation to human well-being, (b) in Nature nothing is worth while but for satisfying a human need interest or preference, (c) it is correct to treat human beings and animals in different ways (Battaghia 1998). 


\footnotetext{
${ }^{14}$ On the primates' capacity to create a proto-culture and consciousness see Vogel G. (1999), Science (284):2070-2073.

${ }^{15}$ It is my opinion that this idea refers to a flawed approach to the problems of animal consciousness: the tendency to read self-consciousness as a mental phenomenon, which must be similar to human beings'. If we are exploring animal consciousness by looking for its similarity with human consciousness, we are not studying aninal consciousness in itself, but are looking for human rationality in organisms that have structural neuro-plysiology and mental properties absolutely unique in themselves.

${ }^{16}$ Another utilitarian argument against oncomouse production would be that mice do not have naturally the induced human patholngy. Thus their genetic transformation would be wrong because it would affect both their welfare and survival. Nevertheless, I believe that this claim, while being extremely important, does not help the dehate on animal biotechnology. We may also use this argunent to assert the moral legitimacy of animal biotechnology. Think of GM animals whose genotype is transformed to enbance their biological potentials. Why should we define this transformation as ethically wrong? Clearly, we do not know whether the transformed animal 'prefers' to be transformed. However, the enhancement of its biological potentials might be interpreted as a possible preference. We thus cannol say whether the preferences of GMOs have been fulfilled, or not.

${ }^{17}$ Both Blackburn (1984) and Mackie (1977) emphasized the dependency of ethics (moral rules) on human culture. Blackburn emphasized the dependency of ethics on social phenomena. and thuman culture. Mackie, on the contrary, emphasized the artificiality of ethics, considered as a set of unjustified "reality's features" that do not exist in thernselves, but which result from a human creation.
} 


\section{II.5. Animal cloning.}

In 1996 the birth of 'Dolly', the first mammal cloned, has opened discussions among biologists and the public about the desirability of such a technology (Terragni 1999, Dijck 1998). The public has plaid a decisive role in decisions associated to cloning both in the US and Europe (Simon 1999). This is surprising when we think that cloning (CL) was not a new technology. The first experiments of nuclear transfer with amphibians (Runa pipiens and Xenoplus laevis) were performed in the United States and Britain during 1950s (Gordon \& Colman 1999:743-746) to study the irreversibility of the modification of genefic material of differentiated cells from adult animals. Nuclear transfer experiments were performed in amphibians in the 1960s, in mice in the $1970 \mathrm{~s}$, in sheep in the $1980 \mathrm{~s}$, and in monkeys in the 1990s. They have provided evidence that fully differentiated somatic cells retain all the genetic material of the early embryo, and that differentiation is almost entirely achieved by reversible changes in gene expression (Wilmut et al. 1997).

In this chapter I will deal with implications arising from animal cloning. The next chapter will address issues related to human reproductive cloning. It is not my intention to minutely report the history of cloning (see Gordon \& Colman 1999:743-746 and Kolata 1998) nor to analyse legal implications that such a technology may involve (on patenting and cloning see Vogel 2000:287). Here I will explore is both the biological and ethical implications of animal cloning ${ }^{1}$.

\subsection{Dolly Superstar.}

'Dolly' is a well-known name among scientists. She is the first mammal to develop from a cell derived from adult tissue ${ }^{2}$. Dolly derived from cells that had been taken from the udder of a 6-year old Finn Dorset ewe and cultured for several weeks in a laboratory. These cells were then fused with unfertilised eggs from which the genetic material had been removed. Biologists cultured 276 of these reconstructed eggs' for 6 days in temporary recipients. Twenty-nine of the eggs that appeared to have developed normally up until the blastocyst stage were implanted into surrogate Scottish Blackface ewes. Dolly's birth drafted a revolutionary way for producing 
engineering animals. In fact, the normal sexual reproduction (the fertilisation of an egg by a sperm and the inter-parental chromosomes fusion) was substituted with a reproductive system by which "DNA is removed from an unfertilised egg and the egg fused with a diploid cell containing a full set of paired chromosomes" (Wilmut, April 1997). Successively, the obtained eggs had been implanted in a surrogate mother, in whose womb they developed into lambs ${ }^{3}$.

The successful experiment performed by members of the Roslin Institute offered a new technique for producing genetically modified animals. Instead of pronuclear injection, which leads to the production of $2-3 \%$ of transgenic animals, the nuclear transfer technique leads to an easier and more successful production of GM animals ${ }^{4}$. To summarise, Dolly's birth has achieved the following scientific goals:

(1) the complete genetic material from an adult mammalian cell has been used in the development of a new individual for the first time; and (2) donor cells, induced to exit the growth phase and become quiescent before being used for nuclear transfer, are more suscoptible to reprogramming by the recipient egg cell and result in the normal developnent and birth of eloned offspring (Campbell et al. 1996, Wilmut at al. 1997).

The following figure illustrates the nuclear transfer technique:

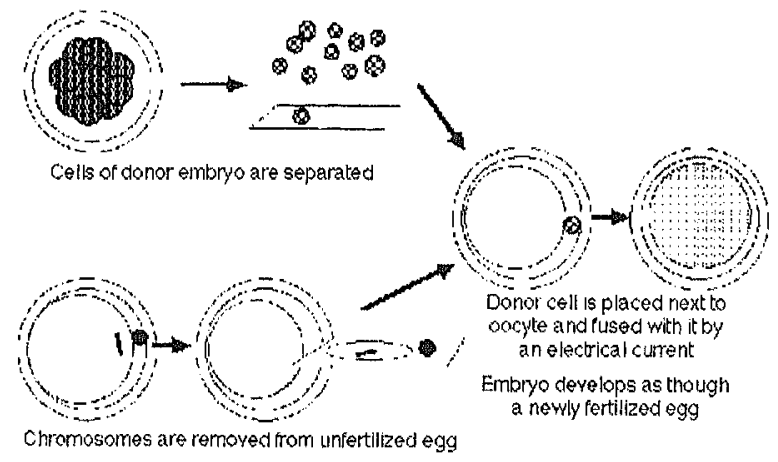

After the successful experiment with Dolly, several laboratories have begun to work on different applications of animal cloning. In 1997, Dr. Pennisi announced the cloning of a transgenic lamb (Polly) cloned from cells engineered with a marker gene and an undisclosed human gene. Dr. Pennisi's experiment showed that "foreign DNA can be introduced to a genome without disrupting the genetic interactions that guide a lamb's 
development" (Pennisi 1997:631). Successively biologists have also begun to clone goats, cattle ${ }^{5}$ and monkey (see Revel, 2000:43-59). Currently a number of experiments are taking place to check whether all animal species are clonable (Cohen 2000:4). Unsuccessful experiments on pigs seem to suggest that not all animal species are clonable (Prather 2000:18861887).

\subsection{Why cloning animals?}

According to the Roslin Institute in Edinburgh animal cloning can achieve a number of scientific goals: 1) Human therapeutic proteins; 2) Organs and tissues for transplants; 3) Nutriceuticals; 4) 'Humanized' cows milk Animal models of disease; 5) Cell therapy agents. In April 1997, Dr Wilmut has summarised the applications of animal cloning as follows:

\section{Applications in Medicine}

\begin{tabular}{|c|c|}
\hline $\begin{array}{l}\text { Human } \\
\text { Therapeutic } \\
\text { proteins }\end{array}$ & $\begin{array}{l}\text { Transgenic sheep, goats and cattle can be used as bioreactor to produce } \\
\text { human protein in milk (for example: alpha- } 1 \text {-antitrypsin). Cloning } \\
\text { could reduce the number of animals needed for creating a transgenic } \\
\text { line }\end{array}$ \\
\hline $\begin{array}{l}\text { Xenotranspl } \\
\text { antation }\end{array}$ & $\begin{array}{l}\text { NTT could lead the production of pigs in which pigs' proteins inducing } \\
\text { rejection are removed and replaced by their human counterparts. }\end{array}$ \\
\hline $\begin{array}{l}\text { Nutriceutical } \\
\text { s }\end{array}$ & NTT ouglat to produce transgenic cows producing human proteins \\
\hline $\begin{array}{l}\text { Animal } \\
\text { models of } \\
\text { disease }\end{array}$ & $\begin{array}{l}\text { Transgenic animals can be produced by using different animal species } \\
\text { than the used ones. 'This extension might allow the creation of better } \\
\text { models for testing treatments for human pathologies }\end{array}$ \\
\hline Cell therapy & $\begin{array}{l}\text { NTT could solve immune rejection problems. We could use cells } \\
\text { removed from a patient and successively reintroduce the patient's cells } \\
\text { (bioengineered) in the organism }\end{array}$ \\
\hline
\end{tabular}

\section{Applications in Biological Research}

\begin{tabular}{l|l}
$\begin{array}{l}\text { Ageing \& } \\
\text { Cancer }\end{array}$ & $\begin{array}{l}\text { NTT avoids DNA replication's mistakes -somatic mutations-and } \\
\text { subsequent ageing and cancer processes }\end{array}$ \\
\hline Alternatives & $\begin{array}{l}\text { Embryo stem cells have only been recovered from two specific strains } \\
\text { of mice (and not from any livestock species). Nucicar transfer would }\end{array}$ \\
to Embryo & $\begin{array}{l}\text { allow gene targeting in other strains of mice and in other laboratory } \\
\text { species such as rabbits and rats. }\end{array}$
\end{tabular}


The ability to produce large numbers of genetically identical animals would have important benefits in experimental design. The advantages of genetic uniformity have been amply demonstrated in studies with inbred lines of mice. Nuclear transfer from culured cells could provide alternative approach in species where repeated inbreeding is impractical or prohibitively expensive.

\section{Gene Targeting in Farm Animals}

\begin{tabular}{l|l}
$\begin{array}{l}\text { Improving } \\
\text { Transgenic }\end{array}$ & $\begin{array}{l}\text { NTT can substitute a procedure called 'pronuclear injection'. The limits } \\
\text { of this technique were: } 2-3 \% \text { of GMOs are transgenic, only a portion of } \\
\text { Animals } \\
\text { transgenic animals completely express the modified exogenous DNA. }\end{array}$ \\
\hline $\begin{array}{l}\text { Opening new } \\
\text { Possibilities }\end{array}$ & $\begin{array}{l}\text { NTT opens the possibility of specific genetic manipulations that are } \\
\text { currently impossible, such as the substitution of a single letter in DNA } \\
\text { (typical of many human genetic diseases) }\end{array}$
\end{tabular}

These potential applications of the nuclear transfer technique show that both animal breeding and pharmacological industry have an interest in animal cloning. The extremely high similarity between clones' genotypes, in fact, would allow manufacturing organisms having improved strains of livestock. The major benefit of cloning, then, is the possibility of creating populations of genetically determined organisms responding to the necds of animal breeding industry or the research community. In parallel, animai cloning may bring to fruition the idea of 'pharming' (using domesticated animals to produce drugs and other medically important substances). Geneticallymodified sheep, goats and cattle are already being used experimentally as 'bioreactors' to produce human proteins in their milk, but cloning should simplify the process of introducing foreign genes into large numbers of animals.

\subsection{Biological implications of cloning.}

Contrary to the common perception, a clone is not a carbon copy of its ancestor. Gene plasticity, environmental factors, and neural topography structures differentiate clones from their parents. Even if clones have a high genetic similarity compared to their parents, the claim that a clone is a carbon copy of another individual is biologically false and it refers to genetic determinism. 


\begin{abstract}
"Yet it seems clear that some ol" these concerns, at least, are based on false beliefs about genetic influence and the nature of the indjviduals that would be produced through cloning. Consider, for instance, the fear that a clone would not be an "individual" but merely a "carbon copy" of someone else - an automaton of the sort familiar from science fiction. As many scientists have pointed out, a clone would not in fact be an identical copy, but more like a delayed identical twin. And just as identical twins are two separate people -biologically, psychologically, morally and legally, though not genetically $-\mathrm{so}$, too, a clone woukd be a separate person from her non-contemporaneous twin. To think otherwise is to embrace a belief in genetic determinism -- the view that genes determine everything about us, and that environmental factors or the random events in human development are insignificant." (Wachbroit R., Genetic Encores: The Ethics of Fuman Cloning, Report for the Institute of Philosophy and Public Policy, 1997)
\end{abstract}

From a biological point of view, a number of biological factors deny the claim that clones are carbon copy of their ancestors (Revel 2000, Shicls et al. 1991): 1) Dolly's mitochondrial DNA comes from the egg donor (usually mitochondrial DNA comes from the mother); 2) Dolly's immune system genes are not developed at the embryo stage (Dolly has a different immune system from her mother). According to biology, environmental factors influence the phenotypic expression of a genotype. "Phenotypic jdentity requires identity between genotypes, which cloning can ensure, and identity between environmental interactions, which it cannot ensure." (Eisemberg, 1999). A cloned DNA, thus, will express differently in the phenotype of different clones. Nevertheless, the similarity between two clones is so high as to show two identical individuals. This coincidence is only apparent, since two clones have a range of constitutive qualitative dissimilarities (Kolata 1998). In 1997, Prof. Hubbard has explained in an editorial published in The Nation (1997) the non-coincidence of cloned individuals. He underlined that "Dolly has the same DNA (or genes) in the nucleus of her genes. But, although embryologists have a way of forgetting it, an egg is not an empty bag containing nothing but a nucleus, transplanted or not. Eggs also contain structural and metabolic equipment, including a complement of extranuclear DNA specific to that individual. The second ewe did not contribute her nucleus, but she did contribute the rest of contents of her egg. The reconstituted egg was than gestation in the uterus of yet another ewe. Dolly is, indeed, a nuclear DNA clone, but there is more to life than DN $A$ for sheep."

Biologically speaking, animal cloning involves a number of implications". We may distinguish two levels of implications; implications for the cloned organism (individual specitic consequences) and implications 
that such a technology may cause at a species-specific level. Let us start with the first kind of implications.

Scientists observed that the mortality of clones is high. Currently, $50 \%$ of clones perish during pregnancy (perinatal mortality). This abnormal perinatal mortality rate may suggest that cloned individuals have physiological weakness ${ }^{7}$ (Cohen 1998:4). Another implication of cloning refers to sexual reproduction mechanisms. A clone develops from a cell derived from an adult tissue. It is not resulting from a chromosomal fusion (maternal / paternal chromosomes) but it derives from a cell of only one organism. As such, clones are anomalous biological individualities since their genotype is a copy of the nucleus of a donor.

In parallel, clones do not have a definite age and we cannot say what parental relation exists with their genitor. As Wilmut noted "One of the more interesting questions about Dolly concerns her age. As far as her DNA goes, is she one year old or seven? And will she age prematurely? The premature death of a number of clones seems to suggest that biologically speaking clones' biological clock runs faster than other animals (Cohen 1999).

At the species-specific level cloning leads to a number of biological implications (ecological and evolutionary implications). The first consequence of cloning would be the impossibility to foresee the long-term consequences that may arise from the use of such a technology. The affirmation of bioengineered gene pools, in a repetitive number, could interact with mechanisms of population genetics by causing the affirmation of specific allelic frequencies that may lead to epidemics or deleterious outcomes towards the Natural evolution (adaptation). We have no ideas about consequences that such a pressure on the Natural evolution may cause. As Prof. Eisemberg has said, "genetic homogeneity is compatible with adaptation to a very narrow ecologic niche; when that niche is perturbed (...) extinction may follow" (Eisemberg 1999:472). Thus, cloning may cause an increase of pathogenic gene frequencies at a population level or the loss of adaptive capacities.

In parallel, a massive use of cloning may seriously damage biodiversity. The continuous mix of genetic data via sexual reproduction is a basic mechanism of the Natural evolution. The possibility of continuously recombining genetic data allows adaptive processes (Gordon 1999 and chapter $I .3$ and $I .4$ of this dissertation). Biologically speaking, the advantage of biodiversity is without controversy. The primary source of genetic variations in living beings is genetic mutation 'and' cell division. The first 
one creates new genetic information that will be naturally selected over time, the second one reshuffles the random genetic changes created by mutations. Clearly a large-scale use of cloning may affect biodiversity and favour the consolidation of specific allelic frequencies at a population level having a negative impact towards the Natural balances. Seen in this light, cloning is intrinsically hazardous.

To summarise, cloning causes a number of biological problems:

1) Clones are not the carbon copy of their parents, but they have an unnatural similarity rate with them so high as to distort the population concept.

Members of a population are reproductively isolated from the others. This clause refers to species-specific similarities (genetic and phenetic similarities) making the reproductive apparatus of species-specific individuals compatible. However, the reproduction-condition among members of a species does not involve their genetic coincidence. Cloning is biologically problematic since it may distort the species-concept.

2) Clones can reproduce and start a cascade of events by which a modified gene lineage could be artificially consolidated at a population level.

Evolutionary mechanisms could be completely affected by cloning. Man could cause the affirmation of gene pools that do not respond to adaptive mechanisms. Clearly, we have no ideas about the evolutionary response to these pressures. Subsequent mutational factors could consolidate gene frequencies (at a population level) that are different from the desired ones.

The above mentioned factors show that cloning is biologically problematic. Nevertheless, we should be aware that these implications concern also other applications of animal genetics (for example, transgenic animals or chimeras). Consequently, I claim that cloning does not posit different ethical implications than the ones of transgenic animals and chimeras. To what extent are we allowed to interact with the Natural evolution to create neworganisms responding to human needs? Are we under the obligation to consider unforeseeable long-term consequences of bioengineering Life as a 'reasonable risk to pay' in the name of the human progress (or economic benefits)? 


\subsection{Animal Cloning.}

To date, a number of research institutes perform animal cloning experiments every day all across the globe. This means that this application of animal biotechnology has been considered morally legitimate. This is because, as I have already said, the main benefit of animal cloning is the more rapid dissemination of genetic progress from elite herds to the commercial farmer. Thus, animal cloning constitutes an ideal tool to optimise both the cost/benefit model and the effectiveness of animal breeding programmes. Let us start to analyse the ethical legitimacy animal cloning by considering the benefits that such a technology may bring forward.

According to $\mathrm{Dr}$ Wooliams "The basic benefit for the biotechnology industry of cloning will (be) ... that it will lead to a high optimisation of engineering organisms' production. (...) Cloning could substitute artificial insemination and embryo transfer in breeding programmes. Farmers could receive cloned embryos of the most productive cows of elite herds. Biotechnology industries could create catalogues that describe the genetic merit of cloned organisms, their fertility, health and longevity ${ }^{8}$ (Woolliams $1997)^{\prime \prime}$.

The first objection to the ethical legitimacy of Woolliams' hypothesis refers to the effectiveness of cloning. The mortality rate of cloning is high. (1 out 277 tries to clone Dolly.) At the present, both the percentage of implanted embryos that had a full-term development $(2.5 \%$, due to failure of genomic reprogramming or imprinting -Revel, 2000:45) and the incidence of perinatal mortality, make this technology really inefficient. However, although this ineffectiveness seems to impede the use such a technology in human genetics (human reproductive cloning), it does not represent a significant obstacle to obstruct animal cloning. We can assume that, in a future, animal cloning will be more afficient (Gordon \& Colman 1999:743746). Or we can argument that other techniques used in animal biotechnology (pronuclear injection, for example) do involve a high mortality rate as well (in transgenic animals production $95-98 \%$ of genetically modified embryos are usually damaged and only $2-3 \%$ of the animals born are transgenic). According to this, as we accept the high mortality rate of producing transgenic animals, we should not think of this side-effect of cloning as a relevant problem. Otherwise we should be in opposition to both animal cloning and transgenic animals production for safety reasons. 
From a philosophical point of view, the capacity to better derive live animals from cultured cells may be considered as a pro cloning argument. In fact, as we think ethically legitimate the production of transgenic animals in both animal industry and research, we should claim the usability of animals in the fields of animal cloning as well. We have seen that animal cloning can increase the effectiveness of transgenic animals production ${ }^{9}$. As such it might be accepted by animal rights advocates as a way to decrease both the mortality rate of animals used in research and animal suffering (clearly in the respect of the animal welfare clause). Ontological approaches to the animal rights issues (Regan's theory, for example), should lead to similar conclusions as well. If animals have an intrinsic value (or a moral status as subjects-of-a-life) the production of newborn organisms (clones) should be considered legitimate in itself. Particularly in those cases where clones inherit a healthy genotype that has not been modified to cause animal suffering (phenotypic expression of the cloned genotype). What arguments can we provide to discuss animal cloning, which differ from the ones posited to discuss the ethical implications of producing transgenic animals? Can we say that two transgenes have a different moral status than two clones? Why? What difference would diversify the moral implications arising from the production of a transgene via pronuclear injection compared to the ones arising from animal cloning?

Cloning is only a technique! The ethical legitimacy of such a technology refers to the conceptual reasons by which biologists and regulatory bodies have justified the moral legitimacy of producing transgenic animals. This does not mean that animal cloning is ethically unproblematic. It simply means that the moral implications of animal cloning can be equated with the ones of manufacturing transgenic animals. Those philosophical theories that do not accept the instrumental use of animals for human purposes will automatically oppose animal cloning. On the contrary, those views that admitted such a use of animals will consider animal cloning as ethically legitimate.

In my opinion, the main problem of animal cloning refers to the ethical legitimacy of using animals for human purposes. And also in this case, my response to this puzzle does not differ from the one I have claimed in the previous chapter of this dissertation: animals are moral entities, but we can instrumentally use them in a restrictive way (in those cases in which the expected goals are so good to justify the use of animals, no alternatives are available and both the number and the suffering of animals is minimised). Then I do not oppose animal cloning, but I wonder whether we really need 
to clone animals. Industrial applications of cloning do not justify animal cloning, since already existing technique used in animal breeding programmes could be used to have improved strains of livestock. On the contrary, research applications of animal cloning having a clear and welldocumented scientific goal (for example to explore the chemical process inducing specific cell differentiation of ES cells), should be allowed. I think that animal cloning is acceptable because of the moral prevalence of human beings in the moral taxonomy (see previous chapter of this dissertation). Clearly, this conclusion strictly refers to the expected outcome of cloning animals. I do not see any moral reason to oppose to animal cloning for research purposes but I also do not see any reason to accept cloning as a technique to use in animal breeding programmes.

\section{NOTES}

'I will explore issues related to clones' identity in the next chapter of this dissertation. Clearly, conclusions cmerging from human clones' identity-puzzles are also applicable to non-humans. The only reason why I will not focus on identity issues here is to avoid duplications.

${ }^{2}$ The invention is covered by two patent applications filed by Roslin Institute (Edinburgh) with a priority date of 31st August 1995: PCT/GB96/02099, entitled Quiescent cell populations for nuclear transfer and $\mathrm{PCT} / \mathrm{GB} 96 / 02098$ entitled Inactivated oocytes as cytoplast recipients for nuclear transfer. Roslin Institute (Edinburgh) does not expect any granted patents to issue for at least another 2-3 years.

${ }^{3}$ As I have already said, this technique was not new. The innovation of the nuclear transfer technique (NTT) was the use of unfertilised eggs and their fusion with a cell that contained the genetic endowment of only one organism.

"In cloning procedures generally, nuclei are extracted from cultured cells that might have come originally from an embryo, a fetus or an adult organism. The nuclei are inserted into egg cells which have had their original nucleus removed, a process called nuclear transfer. In the initial work at the Roslin Institute, the egg cells along with their transplanted nuclei were then implanted directly into a foster mother, where they developed and, in the case of Dolly, resulted in a viable offspring."

(http://www.sciam.com/explorations/090297clone/beardsley.html).

"The NTT technique is actually used in Denmark and Australia to clone catle (Viborg Laboratories of Denmark's National Institute of Animal Science, and Monash University). The Danish team is using genetic material from dead cows. They emptied oocytes of their DNA, take adult cells fron cows' ovaries, they fuse the empty oocyte with the empty cells and tinally implant the oblained blastocyst in a surrogate cow. On this topic see Coghlan A., New Scientist 17, January 1998

"A biological implication of $\mathrm{CL}$ is that it has revolutionised the concept of totipotency. At present, we cannot think of germ cells as the 'only' totipotent cells anymore because NTT 
allows somatic cells to originate a new organism. (ES cells have this capacity as well.) In the light of this, we can think of totipolency as a capacity to differentiate into nearly any cell type.

'This mortality rate may decrease in a future when biologists will get a better understanding of what determinants cause the death of clones during pregnancy.

${ }^{*}$ They could choose the sex of the embryo (male for beef and female for milk) and would be guaranteed a genotype of proven performance in either low or high input systems. The cloned embryo would be delivered to the farm in much the same way as semen straws are today, perhaps from breeding companies based overseas. (John Woolliams, Cloning in farm onimal production, 1997, RIO)

"The claim the cloning does involve a high mortality rate would offer a strong argument against cloning. However, new discoveries of biological sciences (the possibility to use embryonic stem cells rather than eggs -New Scientist, 29 Juanary 2000) can offer technical solutions for this problem. 


\section{II.6 Human Reproductive Clonìng}

In 1998, Dr. Seed, a Chicago physicist declared he would open a clinic for cloning human beings. His declaration referred to results achieved by Dr. Don Jons who announced the cloning of two Rhesus monkeys resulting from 166 attempts using a cell taken from embryos as donor (New Scientist 17, January 1998). Since the monkey's DNA differs from humans' by only $1 \%$ of acid nucleic information (Wakayama 1998, Cohen 1998), Dr Seed declared that the cloning of a human being was practicable.

In December 16, 1998, a team of scientists from South Korea cloned a human embryo. The Korean scientists removed the nucleus of an egg cell donated by a 30 -year-old woman and replaced it with a nucleus taken from her somatic cells. This experiment was stopped when embryo was dividing into 4 cells because of moral concerns. It has proofed that human reproductive cloning is feasible (Eisemberg 1999:471). Finally, an Italian scientist (Dr Antinori) in February 2001 announced that he is ready to clone a human being. The experiment should take place in Israel next year. All these examples show that discussions about human reproductive cloning are needed and urgent.

The following figure shows the technique to use to clone human beings:

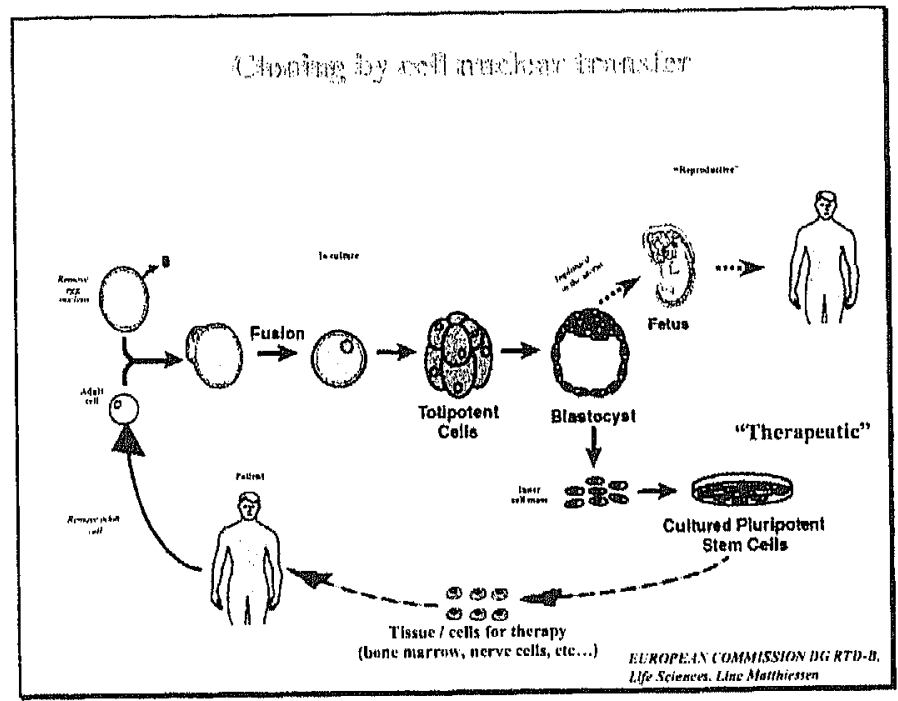


In a report, recently published three general areas of research have been so far identified that would benefit of the use of nuclear transfer cloning, using either embryonic or adult human donor nuclei: (1) basic developmental biology conducted in vitro on embryos up to day 14; (2) in vitro cell differentiation to generate specific human cell types for potential cell-based therapies; and (3) the generation of cloned offspring for the treatment of infertility or related reproductive reasons (Don, 2000, http://www.rand.org.cti). All these data show that human cloning is an extremely promising technology for both bio-medical and pharmacological industry'. As such, if we do not provide valid arguments to reject such a technology we have no reason to oppose to it a priori ${ }^{2}$.

In this chapter I will approach the problem implications of human reproductive cloning (HRC). I will explore the pro-cons arguments that have been posited by biocthicists to discuss HRC. Clearly I will not report the biological implications of HRC and the limits of nuclear transfer technique. Both these topics have been analysed in the chapter II.5 of this dissertation.

In this chapter, however, I will not deal with the so-called therapeutic cloning (cloning of human embryos for research purposes ${ }^{3}$ ). I am aware that the decision of the British Government (if confirmed at a parliamentary level) to allow such a research practice is extremely controversial vis ci vis other European Guidelines (Directive on Biotech patents (98/44/EC), European Parliament resolution on cloning (7.09.2000), the opinions $\mathrm{N}^{\circ}$. 910 of the European Group on Ethics, the additional protocol on Cloning of the Council of Europe, and so on). And I am also aware that this research area is extremely promising for Stem cells research. However, I intentionally will not address issues related to therapeutic cloning because (a) the cloned embryo is not implanted into the uterus of the recipient mother and (b) the embryogenetic process is stopped at an early developmental stage of embryogenesis (4-5 days). At this stage of embryogenesis cells do not differentiate yet and the cloned pre-embryo does not develop. Then, the so-called 'therapeutic cloning' is not a cloning of an organism (such other kinds of cloning for example CPR), and is not a therapy (nobody will be treated). It is an application of stem cell research. Because of therapeutic cloning does not involve the production of an organism, it is out of the scope and the economy of this dissertation. 


\subsection{Human Reproductive Cloning.}

As I previously said, the possibility to clone human beings is concrete. No heuristic limits prevent the use of HRC in human genetics (Colmann 1999; Eisember 1999). Moreover, political reactions to this hypothesis have been so alarmist as to ban cloning. The idea to use cloning to manufacture human beings has been considered as a terrific prospect. In the United States, President Clinton banned human reproductive cloning from both federal financing and privately funded research. The Director-General of the World Health Organization (WHO) defined human cloning as "ethically unacceptable as it would violate some of the basic principles which govern medically assisted reproduction. These include respect for the dignity of the human being and the protection of the security of human genetic material" (WHO 1997). Around the world human cloning was called a violation of human rights and human dignity. In Europe, the Council of Europe had condemned human cloning in 1986. The negative reaction to the use of cloning for human reproduction is exemplified in the opinion produced by the European Group of Experts on ethics (EGE) of the European Commission:

"... Any attempt to produce a genetically identical human individual by nuclear
substitution from a human adult or child cell ('reproductive cloning') should be
prohibited... The use of cloning on humans resurrects the controversy about embryo
research. It brings into play the ethical principles of respect of human dignity and non-
instnumentalization of the human body. It also creates concerns regarding its potential
use for cugenics objectives." (The GAEIB's Opinion No 9 of 28 May 1997)

Although human reproductive cloning is banned in most of the countries of the world ${ }^{4}$, it is possible that in some laboratory scientists are currently cloning a human being. To what extent would such an action be ethically wrong? Let us try to respond to this question through the main arguments posited by bioethicists to oppose human reproductive cloning:

1) Human reproductive cloning would violate human dignity and uniqueness.

Some ethicists claimed that HRC would affect the dignity of human beings (Macklin 1994). They said that we should oppose HRC. because it would diminish the respect of human life (human beings as 
'replaceable'). This is for two reasons: if we clone a specific individual, 1) his/her identity will be duplicated, 2) human beings will be seen as manufacturable. Let us consider the two claims separately.

The first claim (duplication of the cloned organism) is problematic since, as we have seen in the previous chapter, we have no reason to consider clones as carbon copies of their ancestor. The interaction between an individual and his/her environment and other biological phenomena (see chapter II.5) make clones autonomous individualities. Clones are organisms having a high similarity rate (from a genetic point of view) with their progenitor. As such, they can be seen as copies of the cloned individual only through genetic determinism. A theory which is confuted by modern genomics, developmental genetics and other branches of biological sciences (for example neuroscience).

The second claim (instrumentality of human beings) is indubitably correct but it also applies to other reproductive technologies (IVF). According to Macklin, this argument would be relevant for HRC because it may involve a different way to value the moral status of clones. In a sense, that people may consider a clone as less valuable (ethically) than other individuals because (a) his/her genome derives from another organism, (b) his/her existence does not refer to sexual reproduction mechanisms.

In my opinion, this claim is hardly defensible. We may apply this argument to say that people being generated by reproductive technologies could be seen as less valuable as well (from a moral point of view), but this is not he case. In all modern societies, people who had born from reproductive technologies and individuals naturally procreated have the same moral status. This is a fact. Why this ethical discrimination should apply to clones and not to the above mentioned individuals? Let us consider the clain that HRC would affect human dignity.

As I have already said, most of the documents prepared by International and National ethics committees to discuss HRC claim that HRC does not respect human dignity. This remark seems to me nonsensical. I have already said (II.3) that the human dignity concept should not be used to discuss the ethics of human genetics since it is unable to explain the physical existence of human beings. If human dignity is inherent to people's Life (an ontological factor), as existing entities, it should apply to clones as well. This is because clones exist as individuals. If the human dignity principle refers to the human right not 'to have a manipulated genome', cloning would be ethically legitimate since $\mathrm{HRC}$ duplicates and not manipulates a given genotype. We cannot claim that clones are copies of their mother (as genetic determinism 
or essentialism said) because as we have already said in the previous chapters of this dissertation, we have reasons to say that clones are (biologically) autonomous individuals. Both the human dignity and the uniqueness criterion do not provide valid reasons to oppose human reproductive cloning.

2) Human reproductive cloning would diminish genetic diversity, leaving us more vulnerable to disease epidemics, etc.

To me this is a valid argument. Some bioethicists claimed that HRC would not cause any impact on both genetic diversity and adaptive phenomena (or epidemics) because the number of manufactured clones would never 'make a difference' at a population level (population genetics). 1 personally do not agree with this claim. When we say that HCR is ethically legitimate we admit (in principle) the use of this technology at a population level. Conceptually we cannot say that when we manufacture two clones our action is ethically legitimate but when we use this technique to manufacture (say) five clones our action is ethically wrong. As such, I do agree with advocates of the biodiversity criterion (Eisemberg 1976), but I do not see any reason to restrict this argument to human applications of NTT. If we attribute a normative value to biodiversity, both human and animal cloning should be equally prohibited ${ }^{5}$. However, 1 am aware we could solve this problem by fixing a maximum number of clones to be produced via HRC.

3) Human reproductive cloning could lead to the creation of human monsters or freaks.

In my opinion this argument does not apply to HRC. Human reproductive cloning is not a genetic manipulation. It is a technique to duplicate an organism with a given genotype (see II.5). The possibility to create monsters does not belong to human reproductive cloning but to other application of biotechnology (think of the creation of human hybrid ${ }^{6}$ ). As a matter of fact, a number of experiments to create human hybrids are taking place around the globe. For example in October 2000, scientists have submitted an application to the European Patent Office to patent an embryonic pig-human hybrid. The researchers, from two private companies (Stem Cell Sciences in Australia and Biotransplant in America), took a cell from a human foetus, extracted the nucleus and then inserted it into a pig's egg cell. Two embryos were grown to the 32-cell stage of embryogenesis (Sunday Times, 8.10.2000). About 97\% of DNA of the human-pig chimera 
was human, the $3 \%$ of DNA from the pig. The ethical legitimacy of producing such organisms is controversial indeed. The fact that we may use HRC to duplicate the pig-human hybrid does not add anything, from a moral point of view, to ongoing discussions about the ethics of producing human hybrids.

4) Human reproductive cloning would increase social inequalities and use resources to allocate to more important medical and social needs.

The claim that HRC would divert economic resources from other areas of biomedical sciences (Callahan 1993) does not concern HRC but issues related to resources allocation in public health systems. If we claim that public funds should not be used for HRC, then private laboratories could finance HRC clinical trials. This would involve that HRC should be simply excluded from public funds and not banned as it is. To the contrary, one may object that since the technology used to clone individuals (NTT) is quite known and not expansive HRC could be seen as a cheap alternative to infertility treatments (for example). Then, the argument stressing issues related to resources allocation do not really concern HRC.

On the other hand, the claim that HRC would increase social inequalities does not provide any valid reason to oppose/authorise HRC. As a matter of fact, rich classes have access to medical infrastructures that are not accessible to poor people. I do not see any reason to claim that human reproductive cloning would contribute to social inequalities without applying this argument to other infertility treatments. If 'social inequalities' is meant as an unfair access to bio-medical technologies, then it is not HRC to increase such a phenomenon that is inherent to modern capitalistic societies. What could contribute to social inequalities would be the use of HRC for instrumental purposes (such as 'producing' slaves). However, we could use other reproductive techniques, such as in vitro fertilisation, to achieve these goals ${ }^{\text {? }}$.

5) Human reproductive cloning could lead to a new type of family.

This is, I think, a strong argument against HRC. We have no ideas concerning the psychological reaction of the cloned individual in considering his/her biological origin. However, this problem concerns also in-vitro fertilisation and the use of donor sperm. It is thus a problem that strictly characterises new reproductive technologies. According to this cloning would be just another way of providing a couple with a biological child they might otherwise be unable to have (cloning as an infertility 
treatment ${ }^{8}$ ). If this claim is acceptable, cloning should be acceptable as well.' (Wachbroit 1997) The central novelty of HRC is the fact that a clone has only one biological parent. This problem might make family lineage unclear. Nevertheless some assisted reproductive technologies, such as anonymous sperm donor, or surrogate maternity, raise similar implications. It is a fact that new reproductive technologies are completely changing the family structure (Charlesworth 1993:65). Why do we have to accept these technologies and refute $\mathrm{HRC}$ ?

6) Human reproductive cloning would lead to unacceptable consequences and risks for the clones.

In the previous chapter we have seen that (a) the perinatal mortality of clones is high, (b) clones have biological defectiveness, (c) cloning is an ineffective technique indeed (276 failures to manufacture Dolly). If we claim that HRC should be prohibited because of safety issues towards both the two actors involved in HRC (the recipient mother and the clone), we should explain why these factors are so relevant to induce us to consider $\mathrm{HRC}$ unethical. On the one hand the recipient mother in whom uterus clones are implanted to develop does not pay different risks that the ones involved in other reproductive technologies (such as IVF). On the other hand, if we have the duty to protect the Life of future-born individuals, we should oppose HRC because of the perinatal mortality rate, but we should extend this argument to IVF as well. As such, both HRC and IVF should be equally prohibited for safety reasons towards the future born individuals.

If we claim that future born clones may have some biological dysfunctions (genetic predisposition to cancer -Weiss 1997, shorter length of Life Cohen 1998:4), we may say that unavoidable risks are inherent to other medical practices involving unborn individuals (in utero interventions to treat spina bifida for example). As such it would be difficult to say whether this argument would really apply to HRC when animal experiments could optimise this technology and explain factors causing the physiological weakness of clones.

\section{7) Human reproductive cloning would violate human rights.}

Bioethicists claimed that HRC would violate a number of human 'rights': the right to a unique genetic identity (Chadwick 1982), the right to ignorance (Jonas 1974), the right to an open future (Feinberg 1980) etc. Why? Let us deal with all these rights singularly, we will see that they all involve genetic determinism. 
Although the genetic identity uniqueness is a fundamental feature of living organisms (see section one of this dissertation) the claim that it should be protected as a human right implies that it is considered as a key biological feature of individuals. Otherwise we would unable to say why we have to respect the genetic identity and not, for example, the metabolic process of a given subject. Also metabolism has a primary role in the biological existence of living beings, but we do use drugs to modify this biological mechanism also for aesthetic purposes (diets, body building etc.). Why should we protect genetic uniqueness and not metabolic uniqueness? We may argue that the genetic makeup of an individual is so important as to be protected as a human right. However, we are required then to proof that this factor is the most important element of living being's individualities. But this is not the case. We have seen that the gene-eye perspective does not have any scientific and logic validity. We do know that although genes play a key role in an organism's biology, they cannot be considered as fundamental factors of Life. In my organic unity theory, I claim that genes are important because of their role during embryogenesis: to unify the organic unity at a certain time (in this case embryogenesis) and trough time. This means that the genetic identity should not necessarily be protected as a human right since DNA per se is not the basic factor of Life. As concerns the other two rights mentioned above (right to ignorance, right to an open future) in my opinion they hardly apply to HRC. Jonas (1974) posited the right to ignorance. According to Jonas homozygous twins have different biographies from the beginning of their existence. Clones would know too much, from a genetic point of view about their future since they may be in contact with the cloned individual. This may provide them with information related to their biological future. To me this remark is nonsensical. As we know, clones will have a different phenotypic expression than the cloned organism. Their biography is not linked to the one of the cloned organism both biologically and psychologically. As such I do not understand why clones may be negatively affected by having contacts with their ancestor.

Also the right to an open future can hardly apply to HRC. Clones have an open future as well. Biologically and biographically they will live autonomous existences, like all human beings do. These lives will be influenced by socio-cultural and environmental factors. Both the right to ignorance and the right to an open future could be relevant if we think of people's existence through genetic determinism. In this case the high similarity among clones would affect their lives. But this is biologically nonsensical. 
The above-mentioned remarks show the extreme problematic nature of human reproductive cloning. Criticisms to HRC do not really provide valid reasons to oppose HRC and accept other reproductive technologies (basically those ones that involve a genetic manipulation of newborn children). The open question is: 'Why should we clone human beings?' Let us consider some potential pro arguments claimed by advocates of HRC:

1) We might clone exceptional individuals (such as Nobel Prize laureates)

The claim that cloning would allow the duplication of exceptional individuals is biologically false. Cognitive sciences and neurology have proved the context-dependency of individuals' intellectual capacities. A clone of Einstein can be an individual with an absolutely normal intelligence. However, the Nobel Prize spermbank is an example that the idea that specific gene pools can be depository of the features of their donors is still consolidated among biologists (see chapter II).

2) We might help those families in which a husband or a wife has a lethal recessive gene ${ }^{9}$

The claim that cloning would obstruct the transmission of specific pathogenic allelic frequency is justified but it could be criticised by saying that those families that are in the condition described above could adopt children rather than use HRC. In parallel, since we have alternative techniques (such as sperm donor and in vitro fertilisation) to solve this problem, why should we use cloning ${ }^{10}$ ?

3) We might substitute existing reproductive technologies maybe with less risk reproductive technique (cloning as an infertility treatment)

The claim that HRC could be conceived as an alternative method to optimise already-existing new-reproductive technologies is extremely problematic. As Dr Brock underlined, the reproductive interest in question (with HRC) is not only reproduction itself but also "a more specific interest in choosing what kind of children to have" (Brock 2000, p.5). As a matter of fact parents have a sort of control of what kind of children to generate (think of prenatal genetic testing) and what kind of person to educate (think of both educational and environmental influences -Harris 1992, 1998). But we cannot deny that HRC has specific features that diversify such a reproductive technology from sexual reproduction. When we perform HRC we do manufacture an individual that has a specific genetic lineage. As 
such, it is hard to me to consider HRC as an alternative to infertility treatments. Even if I do see that a number of infertility treatments and HRC rise similar moral implications ${ }^{11}$.

4) We might use HRC for obtaining life-saving organs.

Dr Kahan (1989) proposed to think of HRC as a way to produce brain-death organisms (brain cells of cloned embryos were supposed to be removed during embryogenesis). It is clear that such a proposal is unethical since it would involve the creation of organisms not only created for instrumental purposes but also being biologically manufactured to have physiological deficits. These deficits would be so deep to unable them to have a psychological identity. I do not see any argument to support this hypothesis. The intentional production of anencephalic newborn is unjustifiable since it would involve the creation of an individual whose existence is intentionally designed for the benefit of another. I am aware that other examples of instrumeantalised selection of embryos exist. For example on March 2000, a child was conceived as a donor. Doctors selected one among six embryos during in vitro fertilisation using early-stage genetic testing. The embryo selection operated on the basis of two criteria: (a) to ensure that a rare genetic disease called Fanconi anaemia would not be inherited and (b) to guarantee a good transplant match for the embryo's to be 6-year-old sister, who does have the disease. But the problem of using HRC to manufacture anencephalics is ethically unjustifiable since the new born will be not only conceived as a donor (as the previous case in which the donor was healthy and the donation itself would not cause any consequence for him), but as an unconscious individual. Such an individual would be intentionally created to have a lack of consciousness capacities. He/she would be intentionally manufactured to have serious handicaps.

All the remarks above mentioned show that the potential benefits of HRC do not provide decisive arguments to support the ethical legitimacy of such a medical practice. To me the main puzzle remains the following: why ought we to clone human beings (such as other living beings)? The use of this technique in reproduction is really problematic since other techniques exist that can be used to help sterile couples. I thus do not see valid reasons to clone human beings. But this does not mean that cloning is wrong, simply that its use is not necessary. 


\subsection{Cloning and Personal Identity problems.}

Here I attempt to emphasise problem implications of cloning towards identity-problems. I will claim that from a philosophical point of view we have no reason to consider clones as copies of the cloned organism. If this has been explained from a biological point of view in the previous pages of this dissertation (see also my analysis on monozygotic twins ${ }^{12}$ ), philosophical arguments to support such a conclusion are still unclear. Let us thus approach this problem via an analytical approach.

If an organism $X$ is cloned (at $t 1$ ), we have some $X$ 's copies ( $\mathrm{Cx}$, at $\mathrm{t} 2$ ) that are not the closest continuer of $X$ (at 12 ), and their number can be changed at our will. But if $\mathrm{CX}$ is not $\mathrm{X}$, who are they? Which identity-continuity links clones to the cloned individual? Suppose two problem cases:

Case a. 'Pasquale $(X)$ is dying, but a moment before he dies, his brain patterns are transferred to three clones $\mathrm{Cx}: \mathrm{P}, \mathrm{Z}, \mathrm{W}$. Upon the completion of the transfer, $\mathrm{X}$ saves himself'

Case b. 'Like (a), but $\mathrm{X}$ dies'.

In Case a we have four entities ( $\mathrm{X}, \mathrm{P}, \mathrm{Z}, \mathrm{W}$ ) with psychological and corporal similarity $(\mathrm{P}, \mathrm{Z}, \mathrm{W}$, have psychological and biological features derived from $X$ ). If $X$ had died (like in the Case $b$ ) we could puzzle which individual of $\mathrm{Cx}$ class continues $X$ so strictly to be his closest continuer. X's closest continuer (at $t 1$ ) will be an individual $Y$ (at $t 2$ ) who is not a member of Cx (at t2), and who is correlated with $X$ (at $t 1$ ) more strictly than Cx (at 12 ), because he causally continues $X$ at $t 2$ (being his bodily continuer). $C x$ features are causally related with $X$ (causal continuation with $X$-features) since clones are copies of $X$. Nevertheless, CX members are not X's closest continuers, because they lose the competition of proximity with $Y$. No $C x$ continues X so closely as to respect X's self-continuation.

At this moment two problems arise: 1) if $Z, W, P$, are not $X$, who are they'? 2) If $X$ dies, can we say that $C x$ (or one of his members) continues $X$ (Case b)?

$\mathrm{Z}, \mathrm{P}, \mathrm{W}(\mathrm{Cx})$ have a psychological identity that is derived from $\mathrm{X}$ at $\mathrm{t} 2$ but that continues autonomously to $X$ after 2 . In parallel they have their own bodily identity even if they share with the cloned individual $X$ a highly similar genetic makeup. As such, they are autonomous identities. $\mathrm{Cx}$ individuals will continue through time with their closest continuers. $Z, P, W$ 
possess a shared genotype but their continuers will not be equals because of this reason. Living different lives they will enrich their psychological traits (memories, experiences, feelings) in a different way. Also physically their features will change (suppose that $Z$ will become an alcoholic, $P$ will lose a leg, $Y$ will maintain his body in perfect conditions).

$X$ 's closest continuer (at $t 1$ ) is that individual $Y$ (at $t 2$ ) which has with $X$ the maximum degree of similarity and causal dependence with $X$-features. If $X$ (at $t 2$ ) would continue on $\mathrm{Cx}$ individuals, we would have the collapse of the transitive property of Self-identity, and we would need to conclude that $\mathrm{Cx}$ individuals are identical and they will live the same life (like a multiple identity). But this is impossible (we can think about the subjectivity of perception). Therefore, $\mathrm{X}$ (at $\mathrm{t} 1$ ) does not have in $\mathrm{Cx}$ any closest continuers, and ends. Clones are identities derived by $X$, but they are autonomous identities. This means that clones have rights and a moral value as other people. (This conclusion prevents the use of cloning to multiply embryos previously manipulated for inducing pathologies such as brain death or anencephalics.) A discriminative approach to cloned individuals is thus unjustified. According to the organic unity theory (a) HRC does not affect the causal mechanisms of an organism's existence (like gene manipulations of germ cells) and (b) clones are autonomous individualities. And therefore we have no reasons to say that HRC is ethically wrong (if the cloned organisms do not have induced physiological dysfunctions). Cloning does not involve any outside interaction with the causal unification processes of the organic unity that it cloned. It does not also involve interacting with the identity persistence mechanisms of clones resulting from HRC. As such according to the organic unity theory $\mathrm{HRC}$ is ethically legitimated in itself.

\subsection{Conclusions.}

Let us come back to the question I have previously posited: Is it right or wrong to clone human beings? My answer to this problem is nether neither univocal nor sure. According to reasons above addressed, clones are autonomous individuals, as such they are moral entities. The problem implication of their existence is possession of a common genetic makeup. We may say that this biological anomaly would make a difference for them only if we would use genetic reductionism arguments. But we have seen that we have no reason to do it. Most of the criticisms to HRC could be used to oppose reproduction technologies (such as IVF) which are generally 
accepted worldwide. Thus, I do not see valid arguments to oppose to HRC, even if the public at large has clearly said that HRC should be banned for ethical reasons.

When we realise that clones are autonomous individuals, it becomes clear that they are moral subjects. We cannot deny their value as moral entities in the name of their unnatural birth, because otherwise we should do the same for people whose birth resulted from other reproductive technologies. HRC would be ethically unacceptable if used to produce living organ banks. However, also in this case, it would not be HRC to be ethically wrong but the genetic manipulation of newborn organisms having a genome modified to express physiological weakness or handicaps. This involves that cloning per se is not problematic from an ethical point of view. What is problematic, in my opinion, is the expected goals of $\mathrm{HRC}^{13}$. Which benefit could we achieve via HRC?

At the present, I do not see any concrete benefit that may arise from HRC. I only see a number of implications (such as the impact of such a technology towards human diversity) that per se do not provide arguments to oppose HRC. These problem implications suggest that it would be better to wait until we have a clearer picture of the reason why such a reproductive technology should be allowed. Cloning is a technique, a tool. Its applications will determine its value.

\section{NOTES}

\footnotetext{
1 The application areas of human eloning research so far identified are the following: 1. Nuclear transfer cloning using adult human donor nuclei for basic developmental biological research on early embryos up to 14 days post fertilizalion, but not for ultimate implantation, gestation, and birth. 2. Nuclear transfer cloning using embryonic human donor nuclei for basic developmental biological research using early embryos up to 14 days post fertilization, but not for ultimate implantation, gestation, and birth. 3. Nuclear transfer cloning using adult human donor nuclei for research purposes on in vitro cell-differentiation to generate specilic human cell types for potential cell-based therapies. 4. Nuclear transfer cloning using embryonic human donor nuclei for research purposes on in vitro cell-differentiation to generate specific hurnan cell types for potential cell-based therapies. 5. Nuclear transfer cloning using embryonic human nuclei for research toward generating cloned offspring in the treatment of infertility or rejated reproductive reasons. 6. Nuclear transfer cloning using adult human nuclei for research toward generating cloned offspring in the treatment of infertility or related reproductive reasons.

'Let us not forget that when new reproductive technologies, such as IVF, were introduced as an infertility treatments, some people said that we came into the Frankenstein era. Now, the
} 
above mentioned technologies are used on a daily basis worldwide and they have lead to the birth of healthy bahies.

${ }^{3}$ In December 1998, both the British Human Fertilisation and Embryology Authority (HFEA) and the Human Genetics Advisory Commission (HGAC) approved the cloning of human embryos tor research into treatments. In the reports published by HFEA and $\mathrm{HGAC}$ the culturing of human stem cells created by nuclear transfer technique (NTT) is considered ethically legitimate, since it 'would not involve the destruction of an embryo' (Ridley M. 'Why we must let cloning go ahead' Daily Telegraph 09/12/1998). According to the above mentioned reports, no ethical concerns arise from the so called therapeutic cloning. I personally completely agree with this conclusion.

"Will human cloning be done? Undoubtedly. In large parts of the world, there will be no particular scruples about this method, and in some countries it could produce forcign exchange, especially il banned in the United States and Europe. The technique used in sheep cloning does not require a highly sophisticated laboratory. Since the United States government does not support research on the human embryo, and the United Kingdom, France and Germany have banned cloning (as Canada also may), the research making cloning possible may take place in Asia, Eastern Europe, or the Near East. Much of it may take place in secret." (Werty D., Cloning Humans: Is it Ethical?, Gene Letter 1, lssue 5, March 1997)

${ }^{5}$ As such, cloning should not be allowed as a reproductive tool in animal breading programmes (or in a strict limited number to be equally used for human and animal beings). Which is exactly the main reason why NTT has been developed.

"People might think that questions related human/non-Human hybrid cells are 'science fiction', but this is not truc. On November 1998 the US National Bioethies Advisory Commission (NBAC) had discussed the bioethical implications of the creation of hovine/human hybrid cells. (Scientists have fused the nucleus of a human cell with an $\mathrm{egg}$ cell taken from a cow.) The NBAC has concluded that since this experiment was not aimed to create hybrid embryos, it did not rise ethical concerns. I personally think that this conclusion is simplistic. The eentral problem, in my view, is: to what extent should interspecies hybridisation be permissible? If the creation of hybrid organisms is legitimate, then hybrids should develop. Why producing hybrid cells should be cthically legitimate and letting hybrid embryos develop should not?

${ }^{7}$ The need of making couple as direct actor in decision processes related to genetics and human reproduction has been stressed by McLaren A. (1998:427-431). Accirding to Dr. McLaren, hurnan genetics has social responsibilities. Then systems of statutory regulations become pre-requisite for a correct use of available techrologies in human reproduction.

"A discussion about HRC as an infertility treatment is in Brock, 2000:4-7

"Rather than risk the one in four chance of conceiving a child who will suffer a short and painful existenee, the couple considers the alternatives: to forgo rearing clildren; to adopt; to use prenatal diagnosis and selective abortion; to use donor gametes free of the recessive trait; or to use the cells of one of the adults and attempt to clone a child. To avoid donor gametes and selective abortion, while maintaining a genetic tic to their child, they opt for cloning. Wachbroit R., 1997. On this topic sec: Cloning Human Beings. Report of the U.S. National Bivethics Advisory Commission (NBAC), 1997; and John A. Robertson, (March 1994), Danicl Callahan 1997

${ }^{10}$ The French report over the cloning humans' problem implications (Reponse ou President de la Republique au sujet du clonage reproductif. Juillct 1997) analysed the problem implications of cloning. In a recent article, Kithara-Frisch said: "The French Report calls our 
attention on three kinds of harms that may affect children produced by cloning. First, genetic identity, by causing the same body and face appearance, would deprive the child of an important physical support for her personal identity. (...) Second, the personal autonomy of these children would also be threatened since their biological characteristics would be due not to chance, as in sexual reproduction, but to the choice of the person responsible for the cloning. (...) Morcover, it also calls our attention on how similar ethical problcms may alteady arise from the spreading practice of prenatal and preimplantation genetic screening. For here too, biological characteristics of the offspring are being chosen by the parents, disregarding thereby the autonomy of the children born as a result of this screening." (Kitahara-Frisch 1., "The Children Born from Human Cloning", Eubios Journal of Asian and International Bioethics 8, 1998:83-84)

"Biologically speaking, it would be hard to defend the claim that cloning would be a good alternative to other infertility treatments because of its ineffectiveness. But, again, this is a technical problem and not a moral argument to oppose to HRC.

12 "Monozygotic twins should not be described as 'identical' because there may be mayor discordance within a pair not only in birth weight and personality but in congenital anomalies and even genetic disease." Bryan E. (1998) p.51. See also Wright L. (1997).

13 This conclusion however could be changed if we attribute to biological uniqueness a fundamental value. But when we apply $\mathrm{CL}$ on bacteria cultures, no eritic is made. In these cases we consider the utility of such biotechnological applications. Why are we afraid of the human applications of CL?? Maybe because they bring to mind positive eugenic, or because $\mathrm{CL}$ interests a class of human elements who have a primal position in a hierarchy of values concerning the natural world? 


\section{Conclusions: Part Two.}

Bioethics is a branch of human knowledge, which should help us to have a better understanding of issues related to sciences and medicine and research. These analyses are not speculative because bioethical dilemmas are real. They refer to hard questions of our day-life, such as to decide whether people in irreversible vegetative state should be kept alive, whether mothers should be allowed to have an abortion, whether genetics should be considered as a treatment, etc. All these examples show that people should wonder on bioethical puzzles, and prejudicial, as well as superficial, analyses should be strongly avoided in bioethics. But, as 1 have said, these questions are complex and concrete solutions are hard to be found.

We have seen that, at the present, biotechnology can modify the biological source of living beings. Cloning, germline gene transformations, production of chimeras, are feasible. Technical problems are being solved. The use of these technologies is enormously profitable, and terribly temptuous. Then, it is the moment 'now', to explore the moral implications of these technologies, not when they have been already used.

The lack of critical analyses on the identity problems has induced me to approach this topic. And recent scientific discoveries, allowing the use of the above-mentioned biotechnological applications, have persuaded me to apply the organic unity model to human and animal biotechnology.

The above mentioned cases have a commune factor: they change the biologic features of future-born organisms. They do not change only the natural reproductive mechanisms, such as other new reproductive techniques do (such as in vitro fertilisation, assisted reproduction, etc.); they change the genetic weapon of individuals at a certain time and trough time. They can influence the future individuality of future-born individuals, as well as the future generations'.

'Germline engineering would change every cell in the body. People would no longer have to make do witl haphazard combination of their parent's genome. (...) Humanity would, in effect, take control of its own evolution'. (Tnylor R. 'Superhuman' New Scientist, 3 October, 1998:25)

We have seen that, according to the organic unity model, human germline gene manipulations cause a bifurcation in the continuative existence of living beings ${ }^{1}$. As such, it would be possible to claim the ethical legitimacy of such a use of human genetics only if we were sure that future born 
individuals would get a basic benefit from such manipulations. But these goals are far to be achieved and are biologically debatable.

We have also seen that the organic unity model can be used to rank the different species in the natural world. According to this, the production of transgenic animals, while changing the organisms' identity, can be accepted in a restrictive way. When no alternatives are available, the benefits of using this technique are clear, and the number of manipulated organisms is minimised, we may justify the production of transgenic animals. We have seen that other parameters (such as the animal right concept or the animal welfare) do not address questions related to this technology in its specificity. We also have seen that reproductive cloning does not raise moral concerns as well, because cloned individuals are autonomous entities. But, we have also seen that reasons justifying the use of this technology are hard to find. All these examples have shown that the organic unity model is usable to explore consequences arising from the use of modern biotechnology in human and animal genetics. But it cannot be considered as a model able to completely analyse the moral problem associated with these implications. This is because of their synergetic complexity. As I said in the introduction of this work, normative aims are avoided in this dissertation. I only wanted to show that the organic unity model 'was' a useful tool to use in bioethics. Here, I have not applied the organic unity model to other issues of major significance in bioethics such as, inter alia:

- Alzheimer patients and people having neurological dysfunction in they psychological continuity

- Persistent Vegetative State patients

- Xenotransplantation

- GM organisms

- Brian death patients

I have not addressed these issues because of the economy of this book. And also because they refer to the organism's individuality and not to the future generations. I hope this dissertation has induced readers to puzzle about the problem of individuality at a certain time and trough time. Perhaps, the organic unity model is not the best tool to respond to these dilemmas, but it is a tool to discuss solutions that are not usually discussed among bioethicists. These solutions are debatable and relative. But they attempt to have a different approach on ethics of engineering Life. 


\section{NOTES}

'This conclusion refers to the idea that organisms' existence is a process in which numberless causal factors co-participate into the continuative existence of individuals (by unifying the heterogeneous complexily of organic systems in a variable homogenous entity). 
Epilogue.

The relevance of the organic unity view in theoretical biology.

In 1995, Daniel Dennett began his book on Darwinism saying "this book is largely about science but is not itself a work of science. Science is not done by quoting authorities, however eloquent and eminent, and then evaluating their arguments. (...) There is no such thing as a sound Argument from Authority, but authorities can be persuasive, some time rightly sometime wrongly. I try to sort this all out, and I myself do not understand all the science that is relevant to the theories 1 discuss, but, then, neither do the scientists (...). Interdisciplinary work has its risks." These words apply to this dissertation as well. I am convinced that if we want to address the notion of individuality on its own complexity we do have to face a number of issues that usually belong to different scientific expertises. Genomics, neuroscience, physics, developmental biology, etc. are integral components of a theory of biological individuality. We thus have to use (transversally) all these mediums to formulate a theory on individuality. Clearly it is not easy to use such a horizontal approach (interdisciplinary). On the one hand it is difficult to have an overview of those scientific discoveries that are relevant with regard to the notion of individuality. On the other hand divergent paradigms in theoretical biology complicate the formulation of a theory that may reach a consensus among experts. This is true within a specific scientific discipline and between scientific disciplines. Would a theory on individuality be an arrogant illusion?

I am aware a number of people would agree with this conclusion, but I personally do not. A scientific theory is an interpretative model to provide a rational explanation of the topic it refers to. As such, it has to correlate different mediums into a coherent and rational explanation. We do know that the notion of individuality is a multidimensional problem. We thus have to take into consideration such a complexity to formulate a theory on individuality. If we do not do so we claim simplistic explanations that are destined to collapse when applied to different features of living beings! Such a theory has to a flexible interpretative model where the biologicalphilosophical phenomena can be introduced and discussed. This mechanism is inherent to the diachronic evolution of scientific thought, and it is an intrinsic feature of the epistemology of biological sciences ${ }^{2}$. 
Clearly the complexity of the individuality-problem complicates the formulation of a scientific-philosophic theory on this topic. But I am convinced that we cannot claim a theory on individuality if it is not responsive to what different branches of biological sciences tell us about this topic, from neuroscience to genomics, from embryology to psychology. I am also aware that my interdisciplinary approach risks to be rejected for having used different scientific mediums that hardly interact among each others. However, I do think the main contribution of this dissertation to the ongoing debate about the notion of individuality, in both theoretical biology and philosophy, strictly refers to the interdisciplinary character of this work. Scientists and philosophers are familiar with most of the problems addressed in this dissertation as they had been described and discussed within the specific scientific discipline their expertise refers to. They are often unfamiliar with the implications arising from the above mentioned theories if applied to other scientific branches. This dissertation can provide inputs for considerations based on comparative, trans-disciplinary issues on living beings' existence as individualities.

In philosophy we then have theories claiming the psychological or bodily identity of individuals. In modern biological sciences we have theories claiming the molecular, genetic, metabolic, identity of living beings. We also have theories claiming the neural or topobiological identity of organisms, and so on. The proliferation of these theories follows the growth of the technical capacity to explore the complexity of biological phenomena. But such a growth, while contributing to a better understanding of the micro-complexity of biological phenomena (vertical approach), is not followed by a horizontal approach among different scientific disciplines. In this dissertation I have linked these views in an integrated theory: the organic unity view. The organic unity view is not a new version of the organismic biology theory. It strictly refers to both the physical and phenomenological nature of living beings. As such, it is methodologically and conceptually innovative. The organic unity model that I have claimed here refers to an interdisciplinary approach in which biological as well as philosophical factors have equally been taken into account to explain the dual nature of living beings. It allows a better understanding of the problem of individuality.

Let us consider the relevance of the organic unity model toward the determination of the human genome sequence. As I said, the organic unity theory stresses both the complexity and the variability of living beings. It does not look for a mechanistic mono-dimensional explanation of 
organisms' existence as individuals but thinks of Life as a variable phenomenon. This means that according to the organic unity theory (a) the interactive diachronic nature of organic systems becomes the basic feature of living beings (contrary to biologic mechanistics) and (b) this variable complexity needs to be explained as a unitary (diachronic) process. This approach is perfectly coherent with results emerging from genomics.

As a matter of fact, the sequence of the human genome announced in February 2001 has deeply affected mechanistic explanation of Natural phenomena. According to this discovery we now know that the number of human genes is lower than expected (around 35.000 genes). And this number is two or three time larger than genes of a plant or a worm. (A fly and a human being share $10 \%$ of their genetic makeup.) The complexity of organic systems, thus, is structural rather than quantitative. It strictly refers to both the (complexity of) networks between correlated constituents of an organism and the relationship between an organism and its environment. The organic unity theory explains the complexity of living beings and proposes a 'logic' to explain both the dual nature of living beings and the identity continuity of living beings over time.

\section{The relevance of the organic unity view in bioethics.}

I have already said in the introduction of the section two of this dissertation that I do think that bioethics should focus on biotechnology because this scientific area is extremely powerful and may radically change the entire structure of future society. For example in January 2001, scientists of the Oregon Regional Primate Research Centre created the first genetically modified monkey (ANDi). Dr Schatten and his colleagues inserted a jellyfish gene for fluorescence into 224 unfertilised monkey eggs (see Science (291) p. 39). The birth of the first Rhesus monkey has shown that it might be possible to use such technology to genetically modify humans. Clearly the technique used is far from being safe or effective. But such technical limits can (will) be quickly solved and do not provide 'real' arguments to oppose the production of $\mathrm{GM}$ human beings for ethical reasons. Similar consideration can be done for human-animal hybrids, clones and so on.

As a matter of fact we are entering in the biotech century, where food, health, environment, economy will be directly or indirectly affected by biotechnology. The technocratic power of biotechnological industry towards 
our destiny is uncontroversial and can be denied only by those who do not want to face this problem for different purposes. Bioethics should thus address biotechnology in its specificity. The problem is that most of the conceptual tools that had been used in medical ethics (autonomy, beneficence, and justice) hardly apply to biotechnology. We thus need new interpretative tools to use to discuss the ethics of biotechnology. Such a new approach should be able to investigate both the biological and ethical factors involved in biotechnology ${ }^{3}$. Clearly this dual attitude is not easily achievable in bioethics. On the one hand empirical facts (which constitute the puzzles to be investigated) cannot be equated with empirical methodologies (which constitute a specific approach to the problems). On the other hand, the ethics of biotechnology should equally refer to (and be responsive of) both the empirical-driven and the conceptual-driven approaches. If not we risk claiming bioethical theories that do not have any link with reality or do not say anything about the desirability or social acceptability of the problem they refer to ${ }^{4}$.

Let us consider few examples. We have seen that metaphysical approaches to the moral status of embryos are so conceptual to be hardly applicable in bio-medical sciences (from human reproductive cloning to IVF). We have also seen that similar considerations can be done to discuss consequential approaches (such as utilitarianism) used to explore ethical implications of using gene therapy to enhance or treat human beings (or reasons to abstain using animals in research). If, as Singer claimed (1994), we need a 'new' ethics to discuss the new discoveries of medical sciences (let us think patients in the intensive care units), this new ethics has also to be discovered to discuss biotechnology. But such a new ethics cannot ignore the peculiarities of the topics to analyse. We cannot approach the ethics of biotechnology without being aware of the biological implications of our case studies. This is the reason why in this dissertation I have first explored the scientific implications arising from a number of case studies (from transgenic animals to human reproductive cloning) and subsequently discussed both the argumentative and scientific consistency of the arguments claimed by ethicists to discuss the above mentioned cases. We have seen that some bioethics theories collapse when referred to the biological features of the problem investigated. Thus, the first contribution of this dissertation towards the current bioethics debate is methodological. Through a comparative study based on 'science' and 'ethics' it has been possible to identify those claims that are applicable to discuss the case studies analysed in this dissertation. 
The second contribution of this dissertation refers to the organic unity theory. I have tested the validity of the organic unity model in practical case studies where the identity of organisms was (a) transformed, (b) problematic (from a biological point of view), for two main reasons.

The first one was to see whether the organic unity model was able to critically analyse the moral consequences arising from the use of new technologies which have implications for the biological existencepersistence of GM individuals and clones. I believe that it is meaningless to propose a view on identity that cannot be applied to practical case sudies. When I say 'to be applied' I mean that such a theory should be usable to explain the existence of organisms as individuals during the entire course of their Life, as well as to clarify those problem cases in which the identity of an individual becomes problematic. In other words, it should be able to say us (e.g.) why an amoeba and a human being are two individualities while being different entities, as well as (e.g.) Alzheiner patients or comatoses persist as individuals while being their psychological continuity completely transformed. We have seen that the validity and effectiveness of the organic unity model does not refer only to its conceptual bases but also to its use to discuss the above mentioned problem cases ${ }^{5}$.

The second reason that has encouraged me to use the organic unity theory to discuss the ethical issues of biotechnology is conceptual. We know that biotechnology is now able to (a) produce new organisms and to (b) interact with the biology of newborn individuals. We thus have to consider identity issues as elements to take into account in discussions associated to both the desirability and social acceptance of new technologies. Decision-makers and bioethicists often have described the acceptability of biotechnology through risk assessment or public perception inquires. However, I think that such tools, while being useful to discuss the socio-economic implications of new technologies, do not help the debate about the ethics of biotechnology. In the light of this, I have used the organic unity theory as a moral reference to discuss the ethics of engineering Life. What I have tried to do was to link the implications of human and animal genetics to the 'logic' of individuality through which organic systems exist as biological individualities at certain times and through time. This approach based on biology (and not metaphysics or ontology) has allowed me to approach biotechnology in a way that is usually not used by bioethicists. I have discussed the relevance of arguments provided by ethicists in respect with the ethical legitimacy of new biotechnologies. We have seen that the organic unity theory can provide a framework to analyse the ethical value of some applications of 
biotechnology by explaining the way in which the organisms' persistence is affected by outside factors (gene modification of human and animal germ cells) or the coming into existence of new kinds of biological individualities (human and animal clones). We also have seen that the organic unity theory can explain the value of living beings at different stages of their Life (which is important for discussions about new reproductive technologies and genetics) which gives us a moral taxonomy to evaluate the creation of new organisms. There are a number of applications of the organic unity model that have been not considered in this dissertation. For example a potential application of stem cell research aims at culturing neural-cells (in vitro) to be implanted to Alzheimer patients in order to restore their intellectual capacities. The continuative existence of such people is extremely problematic since the transplant would affect both their psychological and bodily continuity.

Another case study would have referred to the use of cadaveric organs for transplant. The first transplant of the complete hand of a patient from cadaveric organs has been performed in 1999. The patient had psychological difficulties to accept the 'new' hand as part of his own body and asked to cut of the new hand (February 2001). An analysis of the people's bodily persistence towards the use of both cadaver and animal organs (xenotransplants) would help to have a better understanding of issues surrounding the ethics of transplants. Other applications of the organic unity view in bioethics would include the definition of death (brain death), ethics of neurosciences (the persistence of subjects having multiple personalities, e.g.) and so on. I have not addressed these issues because of the economy of this dissertation. But they can be considered as areas to be explored in a future.

The aim of this dissertation was to contribute to the debate about the notion of individuality in theoretical biology, philosophy and bioethics. To my knowledge, no one has ever advocated a theory of individuality through all the biological sciences involved (from genetics to neurobiology, from developmental embryology to evolutionary epistemology). No one has used the closest continuer scheme to explain the biological continuity of individuals. In parallel, no one has ever used the organic unity theory as a framework for the analysis of the ethical issues of biotechnology. This theoretical approach has enabled me to explore ethical issues that go beyond the mere analysis of safety, benefits and risks, which is the usual procedure for ethical technology assessment. I believe that such a theoretical approach is necessary in order to come to a really fundamental assessment of the 
ethical issues that are at stake in the ever expanding and revolutionising world of biotechnology.

\section{NOTES}

'We thus have identity theories based on psychological (and neuro-topological) criteria that do not apply to the first stages of an organism's existence as well as to some specific problematic case-studies (anencephalics, amnestics, Alzheimer, etc.). And we also have theories focusing on specitic constituents of living beings (genes, neurones) that hardly apply to the qualitative features of individuals.

The lack of theories about the notion of individuality in biology and philosophy could be interpreted in two different ways; we may say that technique rather than science dominates 'our' time (following Prolessor' Latour), or we may simply claim that the intelligibility of some Natural phenomena. What is the right answer? As a matter of fact, we can manufacture newbom organisms responding to specific needs (think of bioreactors) but we hardly can explain what organisms are. We have a technical knowledge about the mechanisms of Life but a surprising lack of knowledge about how biological systems work. Two factors explain why theorics on organisms' individuality are rare: 1) The super-specialisation of scientific rescarch obstructs interdisciplinary analyses, since these last ones cannot respect the criteria of scientificness (data, documents, experimental results, etc.). 2) Scientists do not appreciate analyses dealing with the macro-complexity of biological phenomena because such studies do not address issues related to the micro-level of organic systems in their specificity. Both these concerns seem to forget that biological theories should not solve all scientific dilemmas. A theory of individuality is an interpretative model based on the available scientific discoveries, while being something more than a list of scientific data. I think that (1) a scientilic theory should also explore the macro-complexity of scientific puzzles, (2) it is intrinsically limited (science evolves), (3) it is an interpretative model that deals with mediate data. This model looks for a coherent 'explanation' of the available scientific information, but it cannot solve all the pending (somctime technical) questions of scientific research. These factors show the weakness/strength of such theories: interpretative models lead a better understanding of natural phenomena. But the growth of natural science (new discoverics) may cause their collapse. Scientific theories are intrinsically relative and destined to be confuted. Nevertheless, this is a phenomenon which is inherent to the history of science (let us think of the Copernican system).

3 The 'biology' of the problems to examine (technical limits, consequences towards environment, hiosphere, evolution, etc.), considered irrelevant by ethicists in a past, has become a key element to take into account to discuss the ethical implications arising from biotechnology. If this is not done we risk claiming positions that, while being conceptually relevant will not have any impact in decisions associated to a fair use of biotechnology.

${ }^{4}$ For example, sociological analyses (such as EUROBAROMETER) have been often considered as bioethical inquires. However, these studies are silent about the ethical factors involved in specific case studies. For example, the claim that public perception reacts to some 
new technology (e.g. GM Seeds) in a deterninate way does not explain what ethical factors these case studies may involve. These last factors need to be explored in their specificity.

Organisms exist, then we cannot think of their existence as merely a theoretical problem but we should be able to formulate reasonable explanations on their physical and phenomenological nature. 


\section{Bibliography}

1. AAAS Human Inheritable Genetic Modifications Assessing Scientific, Ethical, Religious, and Policy Issues, Frankel M. \& Chapman A. http://www aaas.org/spp/dspp/sfrl/germline/main.htm

2. Agar W. (1951) Contribution to the Theory of Living Organisms $2 \mathrm{~d}$ ed. Melbourne

3. Ageno M. (1986) Le radici della biologia Feltrinelli Milano

4. Akin E. (1983) 'Bifurcation in the two locus genetic model' Memories of the American Mathematical Society (284) pp. 1-190

5. Alpern K. (1992) The Ethics of Reproductive Technology N.Y.: Oxford University Press

6. Altenberg L. (1991) 'Chaos from linear frequency-dependent selection' The American Naturalist (138) pp. 51-68

7. Anderson 1. (1999) 'The pigs must die!' New Scientist, 3 April, 1999:4

8. Anderson J. (1991) The adaptive character of thought Erlabum Hillsdale N.Y.

9. Anderson J. R. (1984) 'The development of self-recognition: A review.' Developmental Psychobiology (17) pp. 35-49.

10. Anderson P. 'Can broken symmetry occur in driven system?' Nicolis G. \&Turner P. (eds.) Order and fuctuation in equilibrium and non-equilibrium statistical mechanics pp. 289-297

11. Anderson W. F. (1984) 'Prospects for human gene therapy' Science (226) pp. 401-409.

12. Anderson W. F. (1989) 'Human Gene Therapy. Why Draw a Line?' Journal of Medicine and Philosophy 14(6) pp. 681-693

13. Anderson W. F. (1994) 'Genetic engineering and our humanness' Human Gene Therapy. 5(6) pp. 755-759

14. Anderson W.F. (1990) 'Genetics and Human Malleability' Hastings. Center Report (Jan/Feb.) pp. 21-24.

15. Anderson, W. F. \& Friedmann T. (1995) 'Gene Therapy: I. Strategies for Gene Therapy' in Reich W. Encyclopedia of Bioethics. Revised Edition, New York pp. Simon \& Schuster Macmillan, pp. 907-914

16. Angier N. (1993) 'Gene therapy begins for fatal lung discase' The N.Y. Times pp. 8

17. Annas G. and Elias S. (1992) eds. Gene Mapping: Using Law and Ethics as Guides Oxford University Press New York

18. Annas G. et all. (1999) 'Stem cell politics, ethics and medical progress' Nature Medicine (5):1339-1341

19. Areen J. (1992) 'The Greatest Rewards and the Heaviest Penalties' Human Gene Therapy 3(3) pp. 277-78 
20. Armentano D, et al. (1987) Virol. (61) pp. $1647-1650$

21. Arnheim R. (1974) Art and Visual Perception University of California Press Berkeley

22. Ayala F. (1987) 'Biological reductionism: the problems and some answers' in Yates F. (eds.) The emergence of order Plenum Press NY pp. 315-324

23. Ayala J. (1974) 'The concept of Biological Progress' Ayala J. \& Dobzhansky T. Studies in Philosophy of Biology University of California Press Berkeley

24. Babad E., Birnbaum M. \& Benne K. D. (1983) The social self: group influences on personal identity. Beverly Hills: Sage Publications.

25. Bagnoli F. \& Lio' P. (1995) 'Selection mutations and codon usage in bacterial modcl' J.Theor.Biol. pp. $271-281$

26. Baird P. (1988) 'Genetic disorder in children and young adults: a population study' American Journal of Human Genetics (42) pp. 677-693 1988

27. Baltimore D. (2001) 'Our genome unveiled' Nature (409): 815-816

28. Barbour V. (2000) 'The balance of risk and benefit in genc-therapy' The Lancet (353) January 29:384

29. Baringa M. (1999) 'The mapmaking mind' in Science (285):189-192

30. Barlow H.B. (1988) 'Ncuroscience: a New Era?' Nature (331) p. 571

31. Barlow J., Cosmides L. \& Tooby J. eds. (1994) The Adapted Mind Oxford University Press N.Y.

32. Bartley W. (1987) 'Theories of Rationality' in Radnitzky (j. eds. Evolutionary Epistemology Rationality and the Sociology of Knowledge Open Court La Salle Illinois pp. 205-214

33. Bassechels M. (1984) Dialectical Thinking and Adult Development Norwood N.Y. Ablex;

34. Battaglia L. (1996) Ehica e Diritti degli Animali Roma-Bari Laterza

35. Bauchamp T.L. \& Childess J.F. (1989) Principles of medical ethics 3rd. ed. N.Y.: Oxford Un. Press

36. Bayertz K. (1994) GenEthics, technological intervention in human reproduction as a philosophical problem Cambridge University Press Cambridge

37. Beckner M. (1959) The Biological Way of Thought N.Y.

38. Beetalanffy L. von (1933) Theories of Development London

39. Begun D. et al. (1992) 'Levels of naturally occurring DNA polyformism correlate with recombination rates' Nature (356) pp. 519-520

40. Benedetti S. et all. (2000) 'Genc Therapy Of Experimental Brain Tumors Using Neural Progenitor Cells' Nature Medicine 6: 447-450..20

41. Berger E. M. \& Gert B. M. (1991) 'Genetic disorders and the ethical status of germ-line gene therapy' Journal of Medicine and Philosophy (16) pp. 667-683

42. Bermúdez J. \& Eilan N. (eds). (1995) The Body and the Self. Cambridge MA: MIT Press.Abstract.

43. Bertenthal B. J. \& Fisher K. W. (1978) 'Development of self recognition in the infant.' Developmental Psychology (14) pp. 44-50. 
44. Beveridge M.C.M., Ross, L.G. \& Stewart J. A. (1997), "The development of mariculture and its implications for biodiversity" in: Morine Biodiversity: Patterns and Processes. (R F. G. Ormond \& J. Gage eds) Cambridge University Press.

45. Billings P. (1999) 'Crossing the Germline' GeneWatch (11) pp.1-4

46. Biro J.I. (1981) 'Persons as corporate entities and corporations as persons' Nature and System 3 pp. 173-80.

47. Blackburn S. (1984) Spreading the world: groundings in the philosophy of language Oxford Clarendon Press

48. Block N. (1995) 'On a confusion about a function of consciousness' Behavioural and Brain Sciences (18) pp. 227-287

49. Bloom F. (2000) 'Breakthroughs 1999' Science (286):2267

50. Boer et all. (1995) 'An ethical evolution of animal biotechnology' Animal science (61):453-463

51. Bohm D. (1957) Causality and Chance in Modern Physics Princeton N.Y.

52. Bond E. (1983) Reason and value London Routledge \& Kegan

53. Bosma (1989) 'Mouse: Characterisation and Potential Uses' Microbiology and Immunology 152

54. Boyd R \& Richerdson P. (1985) Culture and the Evolutionary Process University of Chicago Press

55. Boyrd R. \& Silk J. (1996) How humans evolved NY Norton

56. Brandom R. \& Burian R, eds. (1984) Genes, Organisms, Populations: Controversies over the Units of Selection MIT Press, Cambridge MA

57. Brandom R. (1990) Adaption and the Environment Princeton University Press Princeton NY

58. Braude S. F. Graham G. \& Lynn S. G. (1995) 'First Person Plural: Multiple Personality and the Philosophy of Mind' Ethics: an international journal of social political and legal philosophy $1053 \mathrm{pp} .655$

59. Brenner S. (1978) 'Six month in category four' Nature (276) pp. 2

60. Bresnam J. (1982) The Mental Representation of Grammatical Relations MIT Press Cambridge Mass

61. Brewer B. (1995) 'Bodily Awareness and the Self' in The Bocty and the Selfed. José Bermúdez Anthony Marcel \& Naomi Eilan. Cambridge MA: MIT Press pp. 291-306.

62. Brock D. (2000) Cloning Human Beings, An Assessment of the Ethical Issues Pro and Con Commissioned Paper Brown University

63. Brom F. W. (1999) 'The use of intrinsic value in the Netherlands' in Dol et al. Recognizing the intrinsic value of animals Van Gourcum: 15-29

64. Broom D. (1998) 'The effects of biotechnology on animal welfare' in Animal biotechnology and ethics eds. Holland A. é Jhonson A., Chapman \& Hall, London

65. Brown J.R. (1977) Perception theory and commitment. The new philosophy of science Precedent publishing 
66. Brunner J.S. (1978) 'Learning how to do Things with Words' in Brunner J.S. \& Garton A. Human Growth and Development Ox ford

67. Brunner J.S. (1981)' The Organisation of Action and the Nature of Adult-Infant Transaction' in d'Ydewalle G. \& Lenz W. Cognition in Human Motivation and Learning Leuven pp.1-13

68. Bryan E. (1998) 'Monozygosis and identity' in Societal, medical and ethical implication of cloning, Luxemburg Eur $O P, \mathrm{p} .51$.

69. Buiatti M. (1995)'Il concetto di informazionc alla luce dei nuovi studi di biologia molecolare' in Atti del convegno su ll concetto di informazione nelle scienze della vita SILFS Universita' di Roma 'La Sapienza' Roma 24-25 Novembre

70. Bulfied (1987) 'Manjpolazione genetica degli animali da fattoria' Biotec, Speciale Animali e Piante pp. 8-9

71. Bunge M. (1959) Causality Cambridge University Press Cambridge Mass.

72. Burke K. Zimmerman, (1991) "Human Germ Line Therapy: The Case for Its Development and Use," The Journal of Medicine and Philosophy 16:597.

73. Burley S. (2000) 'An overvicw of structural genomics' 2000) Nature America pp. $932-934$

74. Burma B. (1949) 'The specie concept: a semantic revicw' Evolution (3) pp. 369370.

75. Bumet F. MacFurtlene (1959) The Clonal Selection Theory of Acquired Immunity Canderbilt University Press Nashville Tenn.

76. Buss L. (1987) The Evolution of Individuality Princeton Princeton University Press

77. Butterworth G. (1992) 'Origins of Self in Human Infancy' Psychological Inquiry (2) pp. 103-111

78. Cain A. (1954) Animal Species and Their Evolution London Hutchinson's University Library

79. Callahan D. (1993) 'Perspective on cloning: $A$ threat to individual uniqueness' Los Angeles Times, November 12, 1993, B7.

80. Callahan D. (1997) 'Cloning: The Work not Done' Hastings Center Report September

81. Camp W. \& Gill C. (1943) 'The Structure and Origin of species' Brilannia (4) pp. $323-385$

82. Campbell (1974) 'Downward causation' in Ayala \& Dobzhansky pp. 179-186

83. Campbell J. (1995) 'The Body Image and Self-Consciousness' in The Body and the Self ed. Bermúdez J. et al. Cambridge MA: MIT Press pp, 29-42

84. Campbell N. (1991) 'Some Anutomy and Phisiology' in Sanders K. Moore B. cds. Anencephalics Infants and Brain Death Treatment Options and the Issues of Organ Donation Law Riform Commission of Viuctoria Melbourn;

85. Campbell, S. (1998). Interpreting the Personal: Expression and the Formation of Feeling. Ithaca: Comell University.

86. Cannon W. (1968) The Way of an Investigator N.Y. Hafner Publ.Comp. 
87. Capechi M. (1989) 'Altering the genome by homologous recombination' Nature (344) pp. 105

88. Caplan A, \& Wilson J. (2000) 'The ethical challenge of in utero gene therapy' Nature genetics (24):107

89. Caplan A. (1981) 'Back to class: a note on the ontology of species' Philorophy of Science (48) pp. 130-140

90. Caplan A. (1992) If I were a Rich Man Could I buy a Pancreas? And Other Essays on the Ethics of Health Care Bloomington: Indiana University Press.

91. Capy P. (1998) 'A plastic genome' Nature (396) pp. 522-523

92. Carnap (1956) Meaning and Necessity 2eds. Chicago Phenix Books

93. Castaneda, H. N. (1988). Self-consciousness, demonstrative reference, and selfiscription. In J. E. Tomberlin (Eds.), Philosophical Perspectives, pp. 405-454. Atascadero: Ridgevicw.

94. Cavalieri P. \& Singer P eds. (1993) The Great Ape Project. Equaliny berond Humanity Fourth Estate s.l.

95. Cavalli-Sforza L. \& Fedman M. (1981) Cultural Tramsmission and Evolution Princeton University Press

96. Chadwick R. (1987) 'Cloning' Philowophy (57):201-204.

97. Chadwick R. F. (1987) 'The perfect baby: introduction' in Chadwick Ethics Reproduction and Genetic Comtrol London: Croom Helm pp. 93-135

98. Changeux I.P. (1986) Neuronal Man: The Biology of Mind Oxford University Press N.Y.

99. Changuex J. \& Chavaillon I. (eds.) (1995) Origins of the human brain Oxford University Press NY

100. Chargaff E. (1987) 'Engineered a molecular nightmare' Notur' (327) pp. 199200

101. Charlton H. (1987) 'Putting Genes into hypoganda mice' Trenck in the Neurosciences pp. 229-230:

102. Chomsky N. (1975) Reflections on Language Random Housc N.Y

103. Chomsky N. (1980) Rules and Representation. Columbia University Press N.Y.

104. Christopher R, R. Bjomson, Rodney L. Rietze, Brent A. Reynolds, M. Cristina Magli, and Angelo L. Vescovi (1999) 'Turning Brain into Blond: A Hematopoietic Fate Adopted by Adult Neural Stem Cells in Vivo' Science January 22; $283: 534-5.37$

105. Churchland P. (1986) Neurophilosophy MIT Press, Cambridge MA

106. Clark S. (1991) 'How much selves make me'?" in Cockbum D. eds. Humon beings Cambridge University Press Cambridge

107. Coghlan A. (2000) 'A wild gene chase' New. Scientist, 9 December 2000): 16-17

108. Coglan A. (2000) 'Cloning without embryos' New Scien/ist, 29 January

109. Cohen et all. (1997) 'Birth of Infant after Transfer of Anucleate Donor Oocyte Cyto-plasm into Recipient Eggs," Lancel pp. 350: 186-87

110. Cohen H. (1969) 'Mathematics and Biomedical Sciences' The Mathematical 
Sciences MIT Press. Cambridge pp. 217-231

111. Cohen 0. (2000) EUROGENETHY, regulation of gene therap) in Europe http://93.48.40.240//www/eurogenethy/ eurogenethy.html

112. Cohen P. (1998) 'Cloning by numbers' New Scientist 19/26 December $1998 \mathrm{pp}$. 28-29

113. Cohen P. (1998) 'Death comes too soon' New Scientist 19-26 December pp. 4

114. Cohen P. (1998) 'Like a virgin' New Scientist, 19/26 December 1998-2 January $1999 \mathrm{pp} .36-39$

115. Cohen P. (2000) 'Double trouble' New Scientis/, 5 February 2000:4

116. Collins F. et al. (1998) 'New goals for the US human genome project: 19982003 Science $(282)$ pp. 686

117. Collins S. (1982) Selfess Persons Cambridge University Press, Cambridge

118. Commander H. (2000) 'Biotechnology industry responds to gene therapy death' Nature medicine (6): 118

119. Connor S. (1985) Essemial Medical Cenetics Oxford Blakcwell

120. Connor S. (1998) 'Professor plans cloned babies for childess men' The Independen/ 26.10.1998

121. Continenza B. \& Gagliasso E. (1906) Specic adattamento estruttura Editori Riuniti Roma

122. Cosmides L. (1989) 'Has Natural Selection Shaped How Human Reason?' Cognition (31) pp. $187-276$

123. Cotman M. (1981) 'Synapse replacement in the nervous system of adult vertebrates' Phisiology Revien' (61) pp. 684-784

124. Council for International Organizations of Medical Sciences CIOMS (1991) The Dectaration of Imuama and Reports of the Working Groups, in Humam (jene Therapy 2(2) pp. 123-129, Summer 1991.

125. Council of Europe. Recommendation 1046 (1986) on the Usc of Humam Embryos and Fetuses for Diagnostic: Therapeutic: Scientific: Industrial and Commercial Purposes, 1986.

126. Couzin J. (1998) 'RAC confronts in utero gene therapy proposals' Science vol. 282,2 October 1998:27

127. Cowley F. (1971) "The Identity of a Person and his Body' Joumal of Philosophy (68) pp. 678-683:

128. Cracraft J. (1987) 'Species concept and the Ontology of Evolution' Biology and Philosophy (2) pp.329-346

129, Crich F. (1989) 'Neuronal Edelmasnism' Trends in Neurosciences /2 (7) pp. 240)-248

130. Crosby J. F. (1993) "The Personhood of the Human Embryo' The dounnal of medicine and philosoply (18) $4 \mathrm{pp} .399-418$

131. Darchy Wentword Thomson (1942) On growth and form Cambridge University Press

132. Danasio A. (1994) Descares error: emotion, reason, and the humam brain Putnam NY 
133. Daniels N. (1992) 'Growth Hormone Therapy for Short Stature: Can We Support the Treatment/Enhancement Distinction?' Growth: Genetics and Hormones 8 (Supplement 1) pp. 46-48

134. Darley J. et al. (1984) Psychology' Englewood Cliff Prentice-Hall

135. Dasgupta S. (1999) 'The Stem Cell Debate' GeneW Wtch, February 1999, vol. 12 (2), pp. 16-17;

136. Davies P. (1981) Other Worlds Sinon \& Schuster N.Y.

137. Davis B. 'Germ-line Gene Therapy: Evolutionary and Moral Considerations" Human Gene Therapy (1992) 3:361-365

138. Dawkins R. (1976) The Selfish Gene Oxford University Press Oxford

139. Dawkins R. (1982) The exrended phenotype San Francisco Freeman Press.

140. Dawkins R. (1996) Climbing Moumt Improbable London Viking.

141. de Capua A. \& Frontali N. (1992) 'Epidemiologia delle malattie genetiche' Frontali N. La cicogna tecnologica Ed. Associate Roma pp. $168-169$

142. de Sousat R. (1997) Biological Individuality in htip: w ww chass.utoronto.ca/-sousa/BIOINDIV.html

143. de Sousa, R. (1989) 'Kinds of kinds: Individuality and biological species' International Studies in the Philosoply of Science 3(2):119-35

144. Defaye 1. et al. (1990) Risks Monagement in Biotechnology Technique et Documentation Paris

145. Dennctt \& Hofstadter (1981) The Mind's / Foundation and Reflections on Self and Soul Basic Book N,Y.

146. Dernett \& Kinsboume (1992) "Time and the obsever: the where and when of consciousncss in the brain' Behavioral and Brain Sciences (15) pp. 183-20.

147. Dennett D. (1978) Brainstorm Bradford Books Bradford

148. Dennett D. (1991) Consciousness Explained Boston: Little Brown \& Company: Falagan

149. Dennett D. (1995) Dawin's Dangerous ldea London Penguin

150. Dennett D. (1996) Kinds of Minds Weidnfeld and Nicolson London

151. DeSalle R Linddey D. (1997) The Science of Jurassich Parck and The lost World Basic Book N.Y.

152. Di Francesco M. (1098) L'To e I suoi Sé, identicà personale e scienád della mente. Raffaello Cortina Editore Milano

153. Dickinson \& Balleine (1993) 'Actions and responses: the dual psychology of bchaviour' in Eilanet et al. eds, pp. 277-293

154. Dickinson A. (1980) Contemporary Animal Leaming Theories Cambridge Cambridge Univesity Press

155. Dickman S. (1990) 'Human Gene Therapy: First European Experiment' Nanure' $348(6300)$ pp. 378

150. Dijck J. (1995) Mamufteturing Babies and Public Consent. Debating the New Reprodintive Techmologies, Flondsmills - London. Macmillan Press

157. Dijck J. (1998) Imagenation of Genetics, Huondsmills London. Macmillan Press 
158. Diriac P. (1947) Principles of Quantum Mechanics 3d ed. Ox ford and N.Y.

159. Dol et al. (1999) Recognizing the intrinsic value of animals Van Gorcum \& Comp. Assen

160. Donald M. (1991) Origins of Modern Mind Cambridge University Press, Cambridge

161. Donald S. Rubenstein et all. (1995) 'Germ-Line Therapy to Cure Mitochondrial Disease and Ethics of In Vitro Ovum Nuclear Transplantation Cambridge Quarierly of Healtheare Ethics 4: 316-39.

162. Donaldson M. (1978) Childien's Minds Norton N.Y.

163. Dretske, F. (1995). Naturalising the Mind. Cambridge, MA: MIT Press.

164. Dulbecco R. (1995) / geni e il nostro finturo Milano

165. Dunstan G. (1984) "The moral status of the human embryo: a tradition recalled '.J of Medical Eihies (10) pp. 38-44

166. Dupre' J. (1993) The Dirorder of Things: Metaphysical Foundations of the Disumity of Science Harvard Universily Press Cambridge MA

167. Durante M. \& Geri C. (1986) 'A comparison of genome modifications leading to genetic and epigonetic tumorous transformation in Nicotiniana spp. tissue cullure ber: Genetics (7) pp. $51-64$

168. Dutton (i. (2000) 'Debating gene therapy's future' Genetic Enginecring Vews (1):1-11

169. Dworkin R. (1992) Life's dominion: an argument about abortion and cuthunasia Knopf NY

170. Dworking R. (1987) Taking Rights Serious/y London

171. Eccles J. (1989) Evolution of the Brain. Creation of the Self Routledge London and $N Y$

172. Edelman (i.M. (1988) Topobiology: An Intodation to Molecular Embryology. Basic Books N.Y.

173. Edelman G.M. (1989) The Rememhered Present: A Biological Theory of Consciousness Basic Books N.Y.

174. Edelman (I.M. (1992) Bright Air Brilliant Fire. On the Matter of the Hind Basic Books N.Y.

175. Fdsall J.T. (1981) 'Two aspects of scientific responsibility' Science (212) p. 11-14;

176. Edwards R. G. (1989) Life Before Birth. Reflections on the Embryo Detule London Hutchinson

177. Egidi R. (1987) Emergence reduction and evolutionary epistemology: a commentary' in Radnitzky (i. eds, Evolutionary Epistemology Rationality and the Sociology of Kmow fedge Open Court La Salle llinois pp. 157.161

178. Ehrlich P.R. \& Holm W. (1962) 'Pattems and Populations' Scichtee (137) pp. $1228-1232$

179. Eigen M. \& Schuster P. (1978) The Hypercicle Springer Verlag Berlin

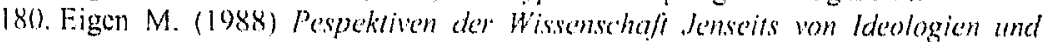
Wunwchdenke'n Deutsche Verlag-Anstal CimbH 
181. Eilan N. (1995) 'Consciousness and the Self' in Bermudez \& Eilan. The Body and the Self Cambridge MA: MIT Press pp. 337-57.

182. Eisemberg L. (1999) 'Would cloned humans really be like sheep?' New England Wumnal of Medicine (340) 6:471.474

183. Eisenberg L. (1076) 'The outcome as cause: Pretestination and human cloning' J Hed Philos, 1:318-331.

184. Elliot R. (1992) Review; "What is a Person?" edited by Michael F. Coodman. Morat Personhood: an Essay in the Philosophy of Moral Psychology by G.E. Scott' Biocthics 6(1) 1992 pp. 41-60

185. Ellot R. (1995) 'Personal Identity Potentiality and Abontion.' Philasophical papers $24(2)$ pp. 141-144;

186. Engehard H.T. (1986) The Foundations of Biouhiex Oxford University Press

187. Engelhardt H. T. (1996) Germ-Line Genetic Engineering and Moral Diversity: Moral Controversies in a Post-Christian Wond Social Philosophy and Policy $13(2)$ pp. $47-62$

188. Epstein F. (1973) 'Metaphysics of Mind and Body Identity' Americen Philosophy Quarterty (10) pp. 111-121.

189. Epsten S. (1991) Regulatory Concerns in Human Gene Therapy' Human Gene Therapt 2(3) pp. $243-249$

100. Ereshefsky $M$ (1991) 'Species Higer Taxa and the Units of Evolution' Philowoln of Scromec (58) pp. 84-10\}

191. Everett H. (1073) The Man-Worlds Interpretations of Quantum Medhanics Princeton University Press Princeton N.Y.

142. Farah M. (1990) Vhatal agnosia MIT Press Canbridge Mass.

193. Feigl \& Meehl (1974) 'The determinism-freedom and body-mind problems' in Schilpp The philosophly of Karl Popper La Salle:Open Court pp. $360-380$

194. Feigl (3. (1980) The Menal and tho Physical Minneapolis University of Minnesota press;

195. Feinberg $\mathrm{J}$. (1980) 'The child's right to an open future' in Whose' Child? Children's Rights. Parenal Auhority; and State Power, W. Aiken, H. LaFollotic (eds.), Totowa, NJ: Rowman and Littlefield

96. Feycrabend P. (1962) 'Explanation Reduction and Empiricism' Minnesola Studies in the Philowophy of Scienc (III) Fleig H. \& Maxwell G. eds. Minneapolis

7. Fiamminghi (1997) Le Scisnce numero speciale 'Bioetica'

4. Finchan J.R. \& Rayer J.J. (1991) Geneticolly Engineered Orgunism.: Risks and Benefits University of Toronto Press. Toronto-Buffalo

- Fisher R. (1930) The Genetic Theory of Natwal Selection Oxford Clarendon Press.

Fletcher 1. (1983) "Moral problems atd ethical issues in prospective human gene therapy' Virginia Lan Revicu (69) pp. $515-540$

Fletcher J. (1988) The Ethices of Genetic Control: Ending Reproducting Roulethe Buffalo NY: Prometheus Books 
202. Fletcher J. (1990) 'Evolution of Ethical Debate about Human Gene Therapy' Human Gene Therapy 1(1) pp. 55-68

203. Fletcher J. C., and Anderson W. F. (1992) 'Germ-Line Gene Therapy: A New Stage of Debate' Law; Medicine and Health Care $20(1-2)$ pp. $26-39$

204. Fluss S. (1998) International guidelines on biocthics, EFGCP News Kessel-Lo.

205. Fodor A. (1974) The Psychology of Langwage McGraw-Hill N.Y.

206. Fodor J. (1981) Representalions: Philosophical Essays on the Foundations of Cognitive Sciences MIT Press Cambridge Mass.

207. Fogel A. (1993) Developing through Relationships: Origins of Communication Self cund Culrure. Hemel Hempstead: Harvester Press;

208. Fowler et al. (1989) The laws of life. Another development and the new biotechnologies' Development Dialogue pp. 13-43

209. Fowter $G$. et al. (1989) 'Germ-Line Gene Therapy and the Clinical Ethos of Medical Genetics" Theorefical Medicine $10 \mathrm{pp}$. 151-165.

210. Fox I. (1999) 'Stem cell hearing stirs biocthics debate' Nature Biolec/nology' 17 January 1999:11

211. Fox. M. (1002) Superpigs and Wondercorm Lyons \& Bufford N.Y.

212. Frege (i. Sense' and Denolation

213. Frey R.G. (1988) "Moral Standing, the Value of Lives, and Speciesism" Berween the Species, Vol. 4. No. 3 (Summer, 1988), pp. 191.201

214. Friedmann T. (1992) 'A Brief History of Gene Therapy' Nature Genetics 2(2) pp. 93-98,

215. Friednann T. (1999) The development of Human Gene Therapy Cold Spring Harbor Press Cold Spring Harbor NY

216. Futreal P. A. et all. (2001) Cancer and (ienome' Nature (409): 850-852

217. Futuyama D. (1982) Science on trial: the case for erolution Pentheam books N.Y.

218. Gallup G. (1982) 'Self awareness and the emergence of mind in prinutes' American . fournal of Primatologe' (2) pp. 237-248.

219. Gallup G. (1907) 'Self-recognition in primates' Americam Psychology (32) pp. $329-338$

220. Calzaniga M. \& Muir A. (1988) "Matematica e Scienza del Vivente" Biologica I La Vita le Forme a i Numeri Transeuropa eds. Bologna pp. 151-168.

221. Gardner W. (1995) 'Can Human Genetic Enhancement Be Prohibited?' Journal of Meticine and Philosophy.20(1) pp. $65-84$

222. Gazzaniga M. (1995) The cognitive nearescicnecs MIT Press Cambridge Mass.

223. Crazzinga M. (1967) 'The Split Brain in Man' Scientific American CCXVll pp. $24-29$

224. Gellatley J. and Wardle T. (1996), The Silem Ark: A Chilling Expose of Meat The Clobal Killer. Thorsons: London.

225. Gene Watch (1909) 12,3. Special lssue 'Eugenics'

226. Ghiselin M. (1966) 'Species concepts individuality and objectivity' Biology and Philosophl' (2) pp. $207-215$ 
227. Ghiselin M. (1974) 'A radical solution to the species problem: Systematic Biolog) (23) pp. 536-44

228. Ghiselin M. (1981) 'Categories life and thinking' Behovioural and Brain Sciences (4) pp. 269-282

229. Ghiseltin M. (1969) The triumph of darwinian methed University of California Press Berkeley

230. Gibbard A. (1990) Wise choices apt Feelings. A Theory of Nomative Jutgement Clarenont Press Oxford

231. Gilbert W. (1992) The vision of Grail Harvard University Press Cambridge

232. Gill et al. (1985) 'Recombinant chicken and bovine growth hormones acceleratc growth in aquacultured juvenile Pacific Salmon Oncarhynchus Kisutch' Biotechnology' 3 pp. 643-646

233. Cilmour J.S. (1940) 'Taxonomy and Philosoply' Huxley eds. The New' Sistematics London: Claredom Press

234. Globus (x. (1973) Consciousness and Brain' Archive General of Psychiatry 29 pp. $153-160$

235. Glover J. (1984). What Sort of People Should There' Be? Genetic: Engineering Brain Control and Their Impact on Our Future World. N.Y.: Penguin Books.

236. Glover J. et al. (1989) Elhics of Nen Reproductive Technologies pp. The Glover Repen to the European Commission. DeKath Northern lllinois Liniversity Press

237. Godfrey-Smith P. and Ruse M. (1998) Complexity and the finction of mind in nature Cambridge University Press Cambridge

238. Gombrich E. (1979) The Sense of Order Cornell University Press thaca

239. Goodman A. (1997) Organic unity theory: an integrative mind-body theory for psychiatry' Theoretical Medicine $184 \mathrm{pp} .357 .378$

240. Goodman N. (1978) Was of Worldmaking Indianapolis

241. Goodwin B. et all. (1983) Development and evolution Cambridge University Press Cambridge

242. Copnik \& Meltroff. (1993) Minds Bodies and Persons: Young Children's Understanding of the Self and Others as Reflected in Imitation and 'Theory of Mind Research' Self-Awareness in Animals and Humans: Developmental Perspectives. Canbridge: Cambridge University Press.

243. Gordon \& Kenneth (1968) The self in social interaction. N.Y.

244. Gordon \& Ruddle (1981) 'Integration and stable germline transmission of genes injected into mouse pronuclei Science $214 \mathrm{pp}$. 1244-1246

245. Gordon J. \& Colman A. (200(0) 'The future of cloning' Nature (402):743-743

246. Gordon J. (1999) 'Genetic enhancement in humans' Science (283):2023-2024

247. Gould S. J. \& Elderedge N. (1972) 'Punctuated equilibria: The tempo and mode of evolution reconsidered Paleobiology (3) pp. 115-151

248. Gould S. J. (1980) Wonderful Life The Burgess Shale and the Nuture of History N.Y.

249. Gridley T. Soriano P. Jaenisch (1987) 'Insertional mutagenesis in mice' T/G 3 
275. Heyes C. (1993) Anectodes, timing trapping and traingulation, do animals attribute mental states? Animal Behaviour (46) pp. 177-188

276. Hilgard E. R. (1977) 'The problem of divided consciousness: A neodissociation interpretation. Amals of the N.Y. Academy of Siciences $296 \mathrm{pp} .48-59$.

277. Hoffman N. (2001) 'Phenotypic profiles determine gene function' Genetic' engineering news (21): $12-13$

278. Hofstadter D.H. (1979) Gocdel Excher and Bach an Extrnal Golden Braid Basic Book N.Y.

279. Holland A. (1991) 'A Fortnight of My Life Is Missing: A Discussion of the Status of the Human 'Pre-Embryo" Appliced Philosophy Almond R. \& Hill D. eds. London: Roulledge pp. 299-311.

280. Holland J. (1998) Emergence from chaos to order Helix Books N.Y.

281. Hollon T. (2001) 'Gene therapy: taking it to the lesion' The Seientist 30 April $2001: 16-17$

282. Holm S. (1994) 'Genetic enginecring and the North-South divide' in Harris I. \& Dyson A. (eds.) ibid. pp. 44-63

283. Holtug N. (1993). 'Human gene therapy: down the slippery slope'?' Bioethic's 7 $402-419$.

284. Holtuman N. A. (1989) Proceed With Caution; Predicting Genetic Risk in the Recombinam DNA Era Baltimore:John Hopkins University Press

285. Holyoak K.J. \& Thagard P. (1995) Mental leaps: Anology in Creative Thromght MIT Press Cambridge Mass.

286. Hope T. (1994) 'Personal Identity and Psychatric Illness' Philosopln: The Jownal of the British Invitute of Philosophical Studies ... p. 1.31

287. Hopper et al. (1987) 'HRPT deficient Mouse Enbryo derived from germline colonisation by cultured cells' Nature 326 pp. 292-295:

288. Hottois G. (1988) Hams Jonas. Nature et responsabilite Amnales de l'Institut de Philosophie de l'Universite' de Bruxelles Paris.

289. Houston \& McNamara (1991) "Genetic covariance of fitness correlates" Evolution (45) pp. 243-253

290. Howell J. (1991) "The History of Eugenics and the Future of Genc Therapy" Journal of Clinical Ethics 2(4) pp. 274-278

291. Hubbard \& Krimsky (1988) 'The Patented Mouse' WATCH (1):6-7

292. Hubbard R. (1986) 'Eugenies and prenatal testing' Intermational J. of the Health Service (16) pp. 227-42

293. Hubbard R. and Wald E. (1993) Exploding the Gene Myth Beacon Press Boston

294. Hull D. (1974) Philosophy of Biological Sciences Englewood Cliffs: PrenticeHall

295. Hull D. (1976) 'A.re species individuals?" Systematic Biolog. (25) pp. 174-191

296. Hull D. (1980) "Individuality and selection" Annual reviow of Ecology and Sisternatics (11) pp. $311-332$

247. Hull D. (1981) 'Units of Evolution' in Brandom \& Burian eds. ('ienes Organisms Populations: Controversies over the Units of Silection MIT Press 
Cambridge MA pp. $142-160$

298. Hull D. (1987) 'Genealogical Actors in Ecological Roles' Biology and Philosophy (2) pp. 168-184

299. Hull R. (1990) Ethical lssues in the New Reprodactive Technologies Belmont: Adsworth

300. Humphrey N. (1992) A history of the mind: erotution and the birth of consciousness Simon \& Shuster NY

301. Hunt \& Krumlauf (1992) 'Hox Code and positional specification in vertebrate embrionic axes" Ann. Rev Cell Biol. (8) pp. 227-256

302. Ito $Y$. (1990) Hypertriglyceridemia as result if human apo Clll gene expression in transgenic mice' Science (249) pp. 790.793

303. Jablonka E. Lamb M. (1995) Epigenetic Inheritance and Evolution. The Lamarkicm Dimension Oxford University Press Oxford

304. Jackson I. (1988) 'No Mickey mouse genetics' Trends in Genetics (4) pp. 273274

305. Jacob F. (1982) The possible and the actual Washington University Press Seattle

306. Jacob L. and Willadsen S. (1997) Birth of Infant after Transfer of Anucleate Donor Oocyte Cyto-plasm into Recipient Eggs' Lancet 350: $186-87$

307. Jaenisch R. and Wilmut I. (2001) 'Don't clone humans' Science (291):2552

308. Jammer M. (1966) The Conceptual Development of Quemum Phusics McGrawHill N.Y.

309. Jansen A. (1969) "How much can we boost 1.Q. and Scholastic achievement?" Harvard Educational Review (4) pp. 1-123

310. Jeftry R. (1965) The Logic of Decisions McGraw-Hill N.Y.

311. Johnson M. (1987) The Bod in the Mind University of Chicago Press Chicago

312. Johnson-Laird, P'. N. (1988). The Comput'r and the Mind an Inroduction to Cognitive Science. Cambridge, Massachusets: Harvard University Press.

313. Jonas H. (1974) Philosophical Essas: From Ancient Cred to Techological Mem, Englewood Cliffs, NJ: Prentice-Hall.

314. Jonas H. (1979) Das Prinaip Verammortung Insel Werlag

315. Jonas H. (1987) 'Crearione dell'uomo' il Mulino (XXXVI) Bologna pp. 615626

$316 . J 013$ son S. (1967) 'Hieratchical clustering schemes' Psuhometrica (32)

317. Jonson V. (1989) 'Contratto e Status Morale' in Eficu e' Animoli p. 85

318 . Jonson-Laird P.T. (1988) The Computer and the Mind Harvard Cambridge University Press

319. Juengst E. (1991) 'Human Germ-Line Engineering' Joumal of Medicine and Philosophy $16(6) \mathrm{pp} .587-694$

320. Juengst E. (1998) 'Should We Treat the Huntan Cierm-Line as a Global Human Resource'? in Enmanuel Aguis and Salvino Busuttil. eds.. (iarm-Linc Intervention and our Responsibilities to Future Generations Great Britain: Kluwer Acadenic Publishers 
321. Juengst E. and LeRoy W. (1995) "Gene Therapy: II. Ethical and Social Issues" in Encyclopedia of Bioethics. Revised Edition, ibid. pp. $914-922$

322. Juengst E et al. (1991) 'Human Germ-Line Engineering' Journal of Medicine and Philosophy $16(6) \mathrm{pp}$. 587-694

323. Juengst, E. (1990) "The NIH "Points to Consider" and the Limits of Human Gene Therapy'. Human Gene Therapy 1(4) pp. 425-433. Winter 1990.

324. Justice J. (1994) Personhood And Death--The Proper Treatment of Anencephalic Organ Donors Under The Law' University of Cincinnati law review" $623 \mathrm{pp}$. $1227-$

325. Kahn J. P. (1991) Commentary on Zohar's 'Prospects for 'genctic therapy, can a person benefit from being altered'? Bioethics. $5(4)$ pp. 312-317.

326. Kaiser M. Wellin S. eds. (1995) Ethical Aspects of Modern Biofectmology Centre for Rescarch Ethics Goeteborg

327. Kanizga G. (1979) Organisation in Vision: Escaty on Gestalt Perception Praeger $N . Y$.

328. Kauffman S. (1992) The origins of orker: self organisation and selection in colution Oxford University Press Oxford

329. Keenan J. (199()) 'What is Morally New in Genctic Manipulation'? Human Gene Therapy $1(3)$ pp. $289-298$

330. Kenneth W. et all. (1999) "Correction of Chromosomal Point Mu-tations in Human Cells with Bifunctional Oligonucleotides," Nature Biotechnology 17: $989-993$

331. Kevles D. (1985) In the Name of Eugenics Knopf N.Y.

332. Khalil E. (1997) Economics, biology and naturalism: three problems coneming the question of individuality' Bology and Philosophy (12) pp. 1852012

333. Khun T. (1977) The essential ension Chicago University of Chicago Press.

334. Kimura M. (1983) The Neutral Theory of Molcular Evolution Cambridge University Press

335. King D; (1996) 'Animal biotechnology' New farmer and pou'er (49):18-19

336. Kitahara-Frisch J. (1998) 'The Children Bom from Human Cloning' Eubios Jounal of Asian and International Biocthics (8) pp. 8,3-84.

337. Kitcher P. (1984) 'Species" Philosophy of Science (52) pp. $308-333$

338. Kitts D. (1984) 'The names of Species' Systcmotic Biology (33) pp. 112-11.5

339. Klotsko A. (1998) 'Immortal Longings' New Scicntist December 5 pp. 49

340. Knowles, Morriw \& Fox (1978) Animal Rights and Human Ethies Washington D.C.: Acropolis Books

341. Koethe 1. (1982) 'The stability of reference over time' Nous pp. 245-52

342. Kotka A. (19.35) The principles of Gestall psychology Harcourt N.Y.

343. Kolak D. \& Martin R. (1987) Personal Identity and Catsality: Becoming Unglued Amorican Philosophical Qharlerly 24 (4)

344. Kolak D. \& Mattin R. (1991) Self and dentity: Contemporary Philosophical Issues Macmillan N.Y. 
345. Kolata G. (1998) Clone: The Road to Dolly \& the Path Ahead Morrow Avon ISBN 0-68815-6924

346. Kolehmainen S. (1999) 'CGR brings ethics into NIH prenatal gene theraphy debate' Genewanch. February 1994, vol. 12 (1). pp. 15-16; Biolechnology News, February 11, 1999:2

347. Krimsky S. (1982) The social history of recombinant DNA controvens, Cambridge, MIT Press

348. Krimsky S. (190) 'Human Gene Therapy: Must We Know Where to Stop Before We Start?' Humam Gene Therapy' 1(2) pp. 171-173

349. Kripke A. (1980) Noming and Necessity Harward Univrsity Press Cambridge MA

350. Kuhen et al. (1987) A potential animal moded for Lesh-Nyan Syndrome through introduction of HTPR mutations in mice' Nature (236) pp. $295-298$

351. Kulm T. (1962) The Siructure of Scientifie Revolutions $2 \mathrm{~d}$ ed. Chicago University Press

352. Kurilo L. (1996) "Nature and Status of embryo, the scientific aspects", Medically assisted precteation and the protection of human embry Council of Europe pp. 8

353. LaCentra D. (1995) Authentic: Self: Toward a Philosophy of Personality Mac millan N.Y.

354. Lakoff G. (1987) Womam. fire and dangerous thing.s University of Chicago Press Chicago

355. Lantos 1. \& Siegler 1. (1989) 'Ethical issues in growth homone therapy'. ,JAMA. 261(7) pp. 1020-1024

356. Lappe M. (1991) 'Ethical issues in manipulating the human germ line' Journal of 'Medicinc and Philosophly (16) pp. 621-639

357. Lebacq\% K. et all. 'Research with Human Embryonic Stem Cells: Ethical Implications The Hastings Center Report 29: $31-36$

358. Lecaldano E. (1970) Le antisi dol lingungyio morde eds. Ateneo Roma

359. Lecaldano E. (1991) Hame e' la nas'ita dell 'alica comemporanea Roma-Bari Laterza.

360). Lecaldano E. (1997) Bioctica c filosofia: il caso della sperimentazione sugli cmbrioni in Berlinguer ( $\mathrm{r}$. (cds.) Lesioni di bioetica, Ediesse edizioni, Romal pp. $57-82$

361. Lecaldano E. (1999) Bioetica Roma-Bari Laterza.

362. Ledley F. (1993) 'Are Contemporary Methods for Somatic Gene Therapy Suitable for Clinical Applications?' Clinical and lmestigative Medicine l6(1) pp. $78-88$

36.3. Ledley R. (1987) 'Somatic gene therapy for human discase: a problem of eugenics?" Trends in Cienerics (3) pp. 112-116

364. LeDoux J. (1996) The cmotional brain Simon \& Shuster NY

365. Lee P. (1990) Personhood the Moral Standing of the Unborn and Abortion Lincure Quenthith $57(2) \mathrm{pp} .8()-89$ 
366. Leenen, H.J.J. (1988) 'Genetic Manipulation with Human Beings' Medicine and Lar $7(1) \mathrm{pp} .71-79$

367. Lemos $\mathrm{N}$. (1994) Intrinsic value Cambridge Cambridge University Press

368. Lengater C. et al. (1998) 'Genetic instabilities in human cancers" Natme (396) pp. 64.3-649

369. LeRoy W. \& Palmer J. (1997) The Ethics of Human Gene Therapy New York and Oxford: Oxford University Press

370. LeRoy W. (1991) 'Ethical lssucs in Human Gene Therapy' Journal of Clinical Ethics 2(4) pp. 267-274

371. LeRoy W. and Palmer J. (1997) The Ethes of Human Gene Therapy Oxford University Press New York

372. Levi 1. (1986) Hard Choices Cambridge University Press Cambridge

373. Levin R. \& Lewontin R. (1985) The Dialectical Biology Harward Univrsity Press Cambridge MA

374. Levine C. ed. (1995) Taking Sides: Clashing View on Controversial Bioethical Issues Guilford, CN: Dushkin

375. Levitrano M. (1986) 'Sperm vectors for introducing foreign DNA into eggs: genetic transformation of mice' $C^{c}$ ll (57) pp. $717-723$

376. Lewin B. (1990) Genes IV Oxford University Press Oxford

377. Lewin R. (1992) Complexity Mac Millan N.Y.

378. Lewis C. (1986) The Abolition of Man London MacMillan

379. Lewis D. (1981) 'Causal Decision Theory' Australium Joumal of Philosophy (59) pp. 5-30

380. Lewontin R. (1974) The genetic basis of evolutionary change Columbia University Press N,Y.

381. Lewontin R. (1998) Gene. Organismo e Ambiente Editori Latersa Roma:

382. Lewontin R.C. (1982) Human Diversity Redding Conn. Scientific American Freeman

383. Liggett S. (2001) 'Pharmacogenetic applications of the human genome project' Nature Medicine (7): $281-282$

384. Lillic R. (1950) Goncral Biology and Philosopty of Organism Chicago

385. Lindemann N. (1995) 'Dethroning Choice: Analogy Personhood and the New Reproductive Technologies' Law' medicine \& health care 23 (2) pp. 129.

386. Lippman A. (1991) 'Prenatal genctic testing and screening: Constructing needs and reinforcing inequities' Americun Journal of Law \& Medicine 17 (1-2) pp. $15-50$

387. Lizza J. P. (1993) 'Multiplc Personality and Personal Identity Revisited.' The British fournal for the Philosophr of Science 442 pp. 263-

388. Lochwood M. (1989) Mind Brain and the Quantum Theory Basil Blakwell Oxford

380. Lock M. \& Lindenbaum eds. (1993) Know/edge Power and Practice: The Anthropology of Medicine and Everyday Life. Berkeley; University of California Press. 
390. Locke J. (1690) Essay concerning Human Understanding

391. Long M. (1993) in Science (260) 1993:91.95

392. Loren R. (1981) Between science and value Columbia University Press

393. Lorenz K (1973) Die ruckseite des spiegels R. Piper \& Co. Verlag-Munchen

394. Lurjia A. (1973) The Working Brain Basic Book N.Y.

395. Lycann W. (1996) Consciousness and experience MIT Press

396. Lyon J. and Gorner P. (1995) Altered Fates: Gene Therapy and the Retooling of Human Life New York Norton

397. Lysons W. (1986) The disappearance of experiences MIT Press

398. Macdonald C. (1989) Mind Body Identity Theories London Routledge \& Kegan;

399. Macer D. (1990) Shaping Genes: Ethics, Law and Science of Using Genetic Technology in Medicine and Agriculture EUBIOS Press. New Zealand

400. Macer D. (1991) 'Whose Genome Project?' Bioethics 5 (3)

401. Mackay D. (1980) 'The indipendence of mind and brain' Neurosciences $5 \mathrm{pp}$. 1389-1391;

402. Mackenzic D. (1990) 'Texas try for customiscd cow' New Scientist 5

403. Mackie J. (1997) Ethics Harmondsworth Penguin

404. Macklin R. (1994) 'Splitting embryos on the slippery slope: Ethics and public policy' Kennedy Institute of Ethics Journal, 4:209-226

405. Madden D. (1998) 'A comparative legal analysis of IVF and the status of human embryos' Biosciences and the Law 1998 (1):387-403

406. Maffettone S. (1992) Le ragioni degli altri Milano il Saggiatore

407. Mancini E. \& Ossicini O. (1999) Bivetica Roma

408. Mancini E. (1996) 'Aspetti sociologici e psicologici delle tecniche di fecondazione assistita' in Bioethics and Humanities (2), Università di Roma La Sapienza

409. Marcel T. and Grausz D. (1996) 'The TMC Worldwide Gene Therapy Enrollment Report' Human Gene Therapy 7(16) pp. 2025-2046

410. Marine Laboratory Abcrdeen (MLA) (1999) Scottish Fish Farms Annual Production Survey, 1998. Fisheries Rescarch Services, Scottish Executive Rural Affairs Department

411. Mark G. (1994) 'Whose Will Is It Anyway? A Discussion of Advance Directives Personal Identity and Consensus in Medical Ethics' Bincthics 8 (1) pp. $27-48$

412. Markova 1. (1990) "The development of self-consciousness: Baldwin Mead and Vygotsky.' J. Faulconcr \& R. Williams (eds) Reconsidering Psychology. Pittsburgh: Duquesne University Press

413. Marshall E. (1998) 'A versatile cell line raises scientific hopes, legal questions' Science (282) 1998:1014-1015

414. Marshall E. (1999) 'Gene therapy death prompts review of adenovirus vector' Science (286):2244-2245 
415. Marshall E. Less H. (1995) 'More Biology Needed for Gene Therapy' Science 270(5243) pp. 1751

416. Marutana \& Varuela (1973) Autopoiesis and Cognition: the Realisation of the Living Boston Boston Studies in Philosophy of Science vol. 42.

417. Marx J. (1989) 'Many gene found in cancer' Science (246) pp. 13861388

418. Matthew G. (1978) 'Animal and the Unity of Psychology' Philosophy LIII

419. Maurer A. (1972) 'Analogy in Early Greek thought' The Encyclopaedia of Philosophy vol. I London Macmillan Publishing Co. \& Free Press pp. 60-67.

420. Mauron A. (1996) 'The human embryo and the relativity of biological individuality' in Evans eds Conceiving the embryo Martinus Nijoff publisher The Hague/London/Boston pp.55-74

421. May R. (1976) 'Simple mathematcal models with very complicatcd dynamics' Nature (261) pp. 459-467

422. Mayr E. (1969) Principles of Systematic Zoology McGraw-Hill N.Y.

423. Mayr E. (1976) 'Is the Species a Class or an Individual?' Systematic Zoology' (25) pp. 192

424. Mays E. (1976) Evolution and the Diversity of Life Cambridge Mass. The Belknap Press Harvard University Press

425. Mayr E. (1982) The Growth of Biological Thought Cambridge Mass. Harvard University Press

426. Mayr E. (1988) Toward a New Philosophy of Biology Harvard University Press Cambridge Mass.

427. Mayr E. Biology and Its History Harvard University Press Cambridge Mass.

428. McCarthy R.A. (1990) Cognitive Neuropshychology: A Clinical Introduction Academic Press N.Y.

429. McCornick R. (1991) 'Who or what is the pre-embryo', Kenncdy Institute of Ethics Journal (1) 1991:1-14;

430. McGee G. (1997) The Perfect Baby: A Pragmatic Approach to Genetics Roswman \& Littlefeld

431. McGee G. (1997) "Therapeutic Clinical Ethics?" HEC Forum, July 1997

432. McGee G. (1997) 'The Not-So-Dcadly Sins of Genetic Enhancement.' in McGee eds. The Perfect Baby: A Pragmatic Approach to Genetics Rowman \& Littlefield London pp. 111-133

433. McGee G. (1998) The Human Cloning Debate Berkeley: Berkeley Hills Press

434. McGinn C. (1983) The subjective view Oxford Clarendon

435. McGinnins \& Krumlauf (1992) 'Homebox genes and axial petterning' Cell (68) pp. 283-302.

436. McGleenan T. (1995) 'Human Gene Therapy and Slippery Slope Arguments' Journal of Medical Ethics $21(6)$ pp. 350-355.

437. Mclntrye (1989) Personal Identity and Passions Journal of the History of Philosophy XXVII pp. 547-557;

438. McKee M. (2000) 'The changing face of bionedical rescarch' Nature Medicine (6):113 
439. McKenny G. (1997) To Relieve the Human Condition: Bioethics Technology and the Body. Albany: SUNY Press.

440. McKusick V. (1988) Mendelian Inheritance in Man Jhon Hopkins University Press

441. McLaren A. (1989) 'IVF: regulation or prohibition?' Nature (342) 1989:6249

442. McLaren A. (1998) 'Genctics in Human Reproduction' Genetics \& Society 14 (10) pp. 427-431

443. Meijsing M. (1997) Awareness self-awareness and perception: an essay on animal consciousness in Van den Bos : 48-61

444. Meins F. (1983) 'Heritable variation in plant cell culture' Ann. Rev. Plant Physiol. (34) pp. $327-346$

445. Mellor D. (1988) 'Natural Kinds' British Journal for the Philosophy of Science (28) pp. 299-312

446. Meltzoff, A. \& Gopnik, A. (1993). The role of imitation in understanding persons and developing a theory of mind. In S. Baron-Cohen, $\mathrm{H}$,

447. Merril G.R. (1980) 'The model-theoretic argument against tealism Philosophy of Science (47) pp. 69-81

448. Mickelson C. et all. (2001) 'The evolution of public review and oversight mechanisms in human gene transfer research: joint roles of the FDA and $\mathrm{NIH}^{\prime}$ Current Opinion in Biotechnology (12): 304-307

449. Mieth D. (1996) 'Gene Therapy - Ethical Implications' 25-27 Symposium on Interdisaciplinary Approaches to Gene Therapy Luneburg

450. Miller H. and Williams W. (1983) Ethics and Animals Clifton New Jersey: Humana Press

451. Mills E. (1993) 'Dividing without reducing: bodily fission and personal identity' Mind (101) pp. 37-51

452. Minsky M. (1986) The society of Mind Simon and Shuster N.Y.

453. Monod J. (1967) Chance and Necessity N.Y. Vintage Books

454. Moore E.F. (1964) 'Mathematics in Biological Sciences' Scient. Amer. (211) pp. 149-167

455. Moore G. E. (1988) Principia Etica Prometeo Books Buffalo N.Y.

456. Moraczewski A. (1991) 'The Human Genome Project and the Catholic Church' International Journal of Bioethics 2(4) pp. 229-234

457. More M (1996) The Diachronic Self Ph.D Thesis Internet (http://primenet.com/ maxmore/).

458. Moreno J. (1999) Undue risk, Secret state experiments on humans Frceman NY

459. Mori M. (1999) 'On the concept of Pre-embryo' in Harris e Holm (eds.) The Future of Human Reproduction Oxford University Press, Oxford pp. 38-54

460. Morowitz. H.J. (1988) 'Rediscovering Mind' Psychology Today August

46I. Morton A (1980) Frames of Mind Oxford University Press N. Y.

462. Morton B. (1959) The Biological Way of Though N.Y,

463. Morton O. (1972) 'Organismic Biology' The Encyclopaedia of Philosophy vol. 1 London Macmillan Publishing Co. \& Frec Press pp. 549-551 
464. Mulligan R. C. (1993) 'The basic science of gene therapy'. Science (260) pp. 926-932

465. Munson R. and Davis L. (1992) 'Germ-Line Gene Therapy and the Medical Imperative' Kennedy Institute of Ethics Journal 2(2) pp. 137-158

466. Murray H. eds. (1996) The Human Genome Project and the Future of Health Care Bloomington and Indianapolis:Indiana University Press

467. Murray T. (1990) 'Human gene therapy the public and public policy' Human Gene Therapy. 1(1) pp. 49-54.

468. Musschenga A. (1999) 'intrinsic value or intrinsic valuing?' in Dol et al. Recognizing the intrinsic value of animals Van Gourcum 1999:97-119

469. Naess A. (1984) 'In defence of deep ecology' Environmental Ethics 6(3) pp. 265-270

470. Naess A. (1989) Ecology, community and life-style Cambridge Cambridge University Press

471. Nagel E. (1951) 'Mechanistic Explanations and Organismic Biology' Philosophy and Phenomenological Research (11) pp. 327-338

472. Nagel E. (1961) The structure of Science Harcourt Brace and World N.Y.

473. Nagel T. (1973) Mortal questions Cambridge University. Press.

474. Nagel T. (1974) 'What Is It Like to Be a Bat?' The Philosophical Review (83) pp. $435-450$

475. Nagel T. (1986) The View from Nowhere Oxford University Press

476. Nelson H. (1995) 'Dethroning Choice: Analogy Personhood and the New Reproductive Technologies' Law medicine \& health care pp. $232-4$

477. Nelson J. R. (1990) 'The Role of Religions in the Aralysis of the Ethical Issues of Human Gene Therapy' Human Gene Therapy 1(1) pp. 43-48

478. Neumann J. von (1955) The Mathematical Functions of Quantum Mechanics Princeton N.Y.

479. Nijhiut et al. (1986) Ontogeny phylogeny and evolution of form: an algorithmic approach' Systematic Biology (35) pp. 445-457

480. Nixon K. \& Wheller Q. (1990) 'An Amplification of the Philogenetic Species Concept' Cladistics (6) pp. 211-213

481. Noble D. (1998) L'éthique du vivant UNESCO

482. Noonan H. (1989) Personal Identity Routledge London

483. Nora J, \& Fraser F. (1974) Medical Genetics: Principles and Practice Henry Kimpton Publisher London

484. Norton B. (1986) Why preserve natural Variety? Princeton University Press Princeton

485. Norton B. (1986) Why preserve natural Variety? Princeton University Press Princeton

486. Nozick R (1981) Philosophical Explanations Oxford University Press

487. Nozick R. (1974) Anarchy State and Utopia Basic Books Inc

488. Nozick R. (1993) The nature of rationality Princeton University Press

489. Nurminsky D. (1998) 'Sclective sweep of a newly evolved sperm-specific gene 
in Drosophila' Nature (396):572:574

490. OCSE (1986) Recombinant DNA Safety Considerations

491. Odegard D. (1969) 'Personal and Bodily ldentity' American Philosophical Quarterly.

492. Old R.W. (1988) Principle of Gene Manipulation Blackwall Oxford

493. Olivetti M. (1992) L'analogia del Soggetto Bari Laterza

494. Olson E. (1997) The Human Animal Personal ldentity Without Psychology Oxford University Press Oxford

495. OTA (1988) Field testing engineered Organisms: genetic and ecological issues

496. Paabo S. (2001) 'The human genome and our view of ourselves' Science (291): 1219-1220

497. Palmiter R, et al. (1982) Dramatic growth of mice develop from eggs microinjected with metallothionein-growth hormone fusion genes Nature 300 : 611-615

498. Parens E. \& Jungest E. (2001) 'Inadvertently crossing the germ line' Science $(292): 397$

499. Parents E. (1995) 'The Goodness of Fragility: On the Prospect of Genetic Technologies Aimed at the Enhancement of Human Capacities' Kennedy Institute of Ethics Journal $5(2)$ pp. 141-152

500. Parents E. (1998) Enhancing Human Traits: Ethical and Social Implications Georgetown University Press

501. Parfit D. (1984) Reasons and persons Oxford University Press

502. Parfit D. (1987) 'Divided Minds and the Nature of Persons' in Blakemore \& Greenficld Mindwaves pp. 19-28

503. Parker S. T. Mitchell R. M. \& Boccia M. L. (1994) Self-Awareness in Animals and Humans: Developmental Perspectives. Cambridge: Cambridge University Press.

504. Paterson D. and Ryder R. eds. (1979) Animals' Rights: A Symposium, Fontwell, Sussex: Centaur

505. Pellionisz A. \& Roesenfeld E. eds. (1988) Neurocomputing: Direction of Research MIT Press Cambridge Mass.

506. Peltonen L. \& McKusick (2001) Dissecting human disease in the postgenomics era' Science (291): 1224-1229

507. Pence G. (1998) Flesh of my Flesh: the Ethics of Cloning Humans Rowman and Littlefield, New York

508. Pence G.E. (1998) Who's Afraid of Human Cloning? Lanham, Rowman \& Littlefield Publishers

509. Penelhum T. (1975) 'Theory of Self revised' Dialogue (94)

510. Penrose R. (1991) The Emperor's New Mind Oxford University Press Oxford

511. Perring C. (1997) 'Degrces of Personhood,' The Journal of Medicine and Philosophy, Vol. 22, No. 2, pp. 173-197

512. Perry J. (1975) Personal ldentity Berkeley University of California Press;

513. Peterfy A. (1995) 'Fetal Viability as a Threshold to Personhood: A Lcgal 
Analysis' The Journal of legal medicine 16 (4) pp. 607-9

514. Peters T. (1995) 'Playing God and Germline Intervention' Journal of Medicine and Philosophy 20(4) pp. 365-386

515. Piaget J. (1971) Biology and Knowledge University of Chicago Press Chicago

516. Pigliucci M. \& Schichting C. (1993) 'Contol of phenotypic plasticity via regulatory genes' The Amertican Naturalist (142) pp. 366-370

517. Pigliucci M. \& Schichting C. (1995) 'Gene regulation quantitative genetics and the reaction of reaction norms' Evolutionary Ecology (9) pp. I54-168

518. Pigliucci M. \& Schichting C. (1996) 'Developmental reaction norms: the interaction among allometry ontogeny and plasticity' Plant Species Biology (11) pp. $69-86$

519. Pigliucci M. \& Schichting C. (1996) 'How organisms respond to enviromental changes: from phenotype to molecules (and vice versa)' Trends in Ecology \& Evolution (11) pp. $168-173$

520. Pigliucci M. \& Schichting C. (1997) ' On the limits of quantitative genetics for the study of phenotypic evolution' Acta Bioteoretica (45) pp. 143-160

521. Pinker S. (1984) Language Learnability and Language Development Harvard University Press Cambridge Mass

522. Pinker S. (1999) How the mind works Norton NY - London

523. Pinker S. (eds.) (1984) Visual cognition MIT Press Cambridge Mass.

524. Plank M. (1932) Where is Science Going? N.Y.

525. Popper K. \& Eccles J. (1977) The Self and Its Brain N.Y.: Springer International

526. Popper K. (1950) 'Indeterminism in the Quantum Physics and in Classical Physics' British Journal for the Philosophy of Science (1) pp. 117-133; 173-195

527. Popper K. (1972) Objective Knowledge Oxford University Press

528. Popper K. (1982) 'Scientific reductionism and the essential incompleteness of all sciences' in The Open Universe: an argument for determinism ed. Bartley Towota Rowmann \& Littleffield pp. 131-62

529. Popper K. (1987) 'Natural Selection and the Emergence of Mind' Radnitzky G. (cds.) Evolutionary Epistemology Rationality and the Sociology of Knowledge Open Court La Salle Illinois pp. 139-153

530. Post S. (1991) 'Selective Abortion and Gene Therapy: Reflections on Human Limits' Human Gene Therapy 2(3) pp. 229-233

531. Powel D. (1987) 'Tecniche transgeniche' Biotec. Speciale Animali e Piante pp. 4.7

532. Premak D. (1983) The Mind of an Ape Norton N.Y.

533. Prigogine \& Stengers (1984) Order Out of Chaos: Man's New dialogue with Nature London Heinemann

534. Prigogine \& Stengers (1987) 'Broken symmetry emergent properties dissipative structures life: are they related?' in Yates E Self-organising system: the emergence of order N.Y,: Plenum Press pp. 445-457

535. Protcor R.N. (1992) Value-free science? Harvard University Press 
536. Provine W. (1986) Sewall Wright and evolutionary biology Chicago University Press Chicago

537. Puccetti R. (1969) 'Brain Transplantation and Personal Identity' Analysis 29 (3) New Series pp. 65-77

538. Purves D. (1988) Body and Brain: a Tropich Theory of Neuronal Connections Harvard University Press Cambridge Mass.

539. Putnam H. (1975) 'The Meaning of Meaning' Putnam H. Mind Language and Reality Cambridge University Press N.Y..

540. Putnam H. (1975) Mind Language and Reality Cambridge University Press N.Y.

541. Putnam H. (1978) Meaning and the Moral Sciences London

542. Putnam H. (1981) Reason Truth and History Cambridge University Press N.Y.

543. Putnam H. (1988) Representation and Reality MIT Press Cambridge Mass.

544. Quine W. (1960) Word and Object Cambridge Mass. MIT Press

545. Quine W. (1969) Ontological Relativity and Other Essays N. Y. Columbia University Prcss

546. Rachels J. (1990) Created from animals: the moral implications of Darwinism Oxford University Press Oxford

547. Radden J. (1996) Divided Minds and Successive Selves: Ethical Issues Disorders of Identity and Personality Cambridge: MIT/Bradford Press.

548. Radnitzky G. \& Bernhold Z, eds. (1987) Economic imperiallsm Pergon House N.Y.

549. Ramachandran, V. \& Hirstein, W. (1997) 'Three laws of qualia: What neurology tells us about the biological functions of consciousness' Journal of Consciousness Studies, 4(5-6), 429-457.

550. Ratain M. \& Relling M. (2001) 'Gazing into a crystal bal-cancer therapy in the post genomic era' Nature Medicine (7): 283-285

551. Rauw W. (1998) 'Undesirable side effects of selection for high production efficiency in farm animals' Livestock production science (56):15-33

552. Regal. P. (1988) 'The Adaptive Potential of Genetically Engineered Organisms in nature' Trends in Biotechnology 6 pp. 36-38

553. Regan T. \& Singer P. (1976) Animal Rights and Human Obligations Englewood Cliffs: Prentice-Hall

554. Regan T. (1983) The Case for Animal Rights Berkeley California.: University of California Press

555. Regine B. (1998) 'Mastcring the game' New Scientist (26 September) 1998:3942

556. Reik W. and Surani A. (1997) Genomic Imprinting Oxford University Press Oxford

557. Reiss M. (2000) 'The ethics of genetic research on intelligence' Bioethics' (14):1-15

558. Resnik D. (1994) 'Debunking the slippery slope argument against human germline gene therapy' Journal of Medicine \& Philosophy. 19(1) pp. 23-40 
559. Revel M. (2000) 'State-of-the-art on Research on Cloning of Whole Organisms', UNESCO IBC Proceedings of the Sixth Session, pp. Errore. Il segnalibro non è definito. -58

560. Richard K. ct all. eds. (1978) Animal Rights and Human Ethics, Washington, D.C.: Acropolis Books

561. Richards R.J. (1987) Darwin and the Emergence of Evolutionary Theories of Mind and Behaviour University of Chicago Press Chicago

562. Rickards T. (1990) Creativity and Problem Solving at Work Gover Aldershot

563. Ridley M. (1985) The Problems of Evolution Oxford University Press

564. Ritter W. E. (1919) The Unity of the Organism 2 vols. Boston

565. Robertson J. (1994) 'The question of human cloning' The Hastings Center Report 24 (2)

566. Robertson J. (1998) 'Liberty Identity and Human Cloning'. Texas Law Review (77) pp. 1-85

567. Robinson D. (1985) 'Can amoebe divide without multiplying?' Australian Journal of Philosophy (63) pp. $299-319$

568. Rochat P. (1995) The Self in Infancy: Theory and Research Amstcrdam: NorthHolland.

569. Roemor I. ct al. (1997) 'Epigenetic inheritance in the mouse' Current Biology (7) pp. 277

570. Rollin B. (1989) The Frankestein Syndrome. Ethical and Social Issues in the Genetic Engineering of Animals Cambridge Cambridge University Press.

571. Rollin B. (1996) 'Bad ethics, good ethics and the genetic engineering of animals in agriculture' Journal of animal sciences (74):535-541

572. Rollin B. (1998) 'On telos and genetic engineering' in Holland and Jonson (cds.) Animal Biotechnology and Ethics Chapman and Hall London 1998:162

573. Rollin B. (1998) The Unheeded Cry. Animal Consciousness Animal Pain and Science Oxford Oxford University Press

574. Rorty R. (1979) Philosophy and the Mirror of Nature Princeton University Press Princeton N.Y.

575. Rose S. (1984) 'Human perfectibility' Lancet (15) pp. 1380-1383

576. Rosenberg S. A. et al. (1990) 'Gene Transfer into Humans-- Immunotherapy of Patients with Advanced Melanoma,Using Tumor-Infiltrating Lymphocytes Modified by Retroviral Gene Transduction' New England Journal of Medicine 323(9) pp. 570-578

577. Rosenkranz E. (1987) 'Custom Kids and the Moral Duty to Genetically Engineer Our children' High Technology Law Journal 2(1) pp. 1-53

578. Ross G., Erickson R. et al. (1996) 'Gene Therapy in the United States: A FiveYear Status Report' Human Gene Therapy 7(14) 1781-1790

579. Rothman B. (1999) Genetic maps and human imagination: the limits of science in understanding who we are W.W. Norton ISBN 0393047032

580. Rottler J. \& Diamond J. (1987) 'What maintains the frequencies of human genetics diseases' Nature (329) pp. 289-290 
581. Rubcnstein D. (1995) 'Germ-Line Therapy to Cure Mitochondrial Disease: Protocol and Ethics of In Vitro Ovum Nuclear Transplantation' Cambridge Quarterly of Healtheare Ethics 4(3) pp. 316-339

582. Rubenstein et all. (1995) 'Germ-Line Therapy to Cure Mitochondrial Disease and Ethics of In Vitro Ovum Nuclear Transplantation' Cambridge Quarterly of Healthcare Ethics 4:316-39

583. Rusc (1973) The Philosophy of Biology London Hutchinson

584. Russ M. (1989) 'Do organisms exist?' American Zoology (29) 1061-1066

585. Russel S. (1991) 'Regulation of Human Gene Therapy in Australia' International Journal of Bioethics 2(2) pp. 87-93

586. Russell E.S. (1938) The Behaviour of Animals 2d. ed. London

587. Salvi M. (1995) 'Epistemologia biologica e bioetica evoluzione molecolare e biotecnologie' Methodologia thought language and methods. Review of the Italian National Research Centre (14 December 1995 pp.33-51).

588. Salvi M. (1995) 'Ethics and biotechnology, analysis of the relation between ethics and science' E.JAIB Journal of Asian and International Bioethics (7) November 1995 pp. 151-3.

589. Salvi M. (1996) 'Biotecnologic c terzo mondo un problema di giustizia' Qualita' Equita' Rivista del welfare del futuro Editrice Libereta' S.r.1, n.3 September pp. 111-123.

590. Salvi M. (1996) 'La responsabilita' della scienza' Bioethics and Humanities University of Rome la Sapienza n.2 Internet 1996 (http://www.uniromal.it/bioetica/home.htm)

591. Salvi M. (1996) 'Lo specchio e il labirinto filosofia analitica e identità personale' Prometeo. Rivista trimestrale di scienze e storia Milano Mondadori ed. Anno 13 n.51 pp. $78-88$.

592. Salvi M. (1996) 'Morte Cerebrale e Problemi di Identita'; la tilosofia analitica del XX secolo e il problema della morte del soggetto' Bioethics and Humanities University' of Rome n.3 Internet, 1996 (http://www.uniromal.it/bioetica/home.htm)

593. Salvi M. (1997) Does Bioethics be an Epistemic Hierarchy or an Interdisciplinar Analysis?' Bioetica Web University de Valparaiso Chile (http://www.imaginativa.cl/ chapa/bioetica.htm)

594. Salvi M. (1997) How Calculating the Utility of Human Germline Gene Transformations? A critic to the Utilitarianism in Biocthics' $E J A I B$ Journal of Asian and International Bioethics (7) March 1997 pp. $36-38$

595. Salvi M. (1997) 'La Biologia come scienza non-paradigmatica' Methodologia thought language and methods Revicw of the Italian National Research Centre n. 17 January 1997.

596. Salvi M. (1997) 'Paradossi Biotecnologici: Identita' Personale e Genetica Applicata' Bioetica rivista interdisciplinare 21997 Milano Franco Angeli eds. pp. 171-192 
597. Salvi M. (1997) 'The Ethical "Neutrality" of Cloning'. Bioetica Web 1997 University de Valparaiso Chile (http://www.geocities.con//Athens/Acropolis/9830/bioctica.htm).

598. Salvi M. (1998) 'Human Genetics and the integrity of Self: Germline Gene Therapy' in D. Macer \& Fujiki N. eds. Bioethics in Asia Cristchurch NZ $1998 \mathrm{pp} .278-284$

599. Salvi M. (1998) 'Ontology and Bioethics, the Case of Human Dignity principle in Human genetics' EJAIB Journal of Asian and International Bioethics 8 (6) November pp. 181-183

600. Salvi M. (1998) Ethics and Germ-line Manipulations: Germ-line transformation of the transgenic mouse, Scientific Report to the European Commission, Programme: Biotechnology

601. Salvi M. (1999) 'Casualita' Biologica Causalita' Biotecnologica' in Bioetica e professione medico veterinaria Quaderni di Bioetica. July 1999 Bologna Macro Edizioni, pp. 109-134

602. Salvi M. (1999) 'Organismi ed Individualità, Controversie sul concetto di individuo in biologia' Prometeo. Rivista trimestrale di scienze e storia Milano Mondadori

603. Salvi M. (2000) 'Biotecnologie e bioetica, un ritorno alla metafisica? Terapia genica in utero, clonazione umana e lo statuto morale dell'embrione.' Bioetica, rivista disciplinare, Roma (foreseen 2002)

604. Salvi M. (2001) "Complessità c bioetica. La bioetica nelle istituzioni curopee' Keiron Roma Farmaindustria pp. 170-175

605. Salvi M. (2001) 'Genetics' Dreams in the post-genomics era' Medicine, Health Care and Philosophy Kluwer Academic Publisher (in press)

606. Salvi M. (2001) 'Le principe de Justice', in Hottois G. \& Missa J.N. (eds.) Nouvelle Encyclopédie de Bioethique, Paris pp.529-531

607. Salvi M. (2001) 'Multiplying individuals, the human reproductive cloning', Ethics and Politics Università di Trieste (in press)

608. Salvi M. (2001) 'Shaping Individuality, Ethics and Human Inheritable Germ Line Genc Modification' Theoretical Medicine, (accepted for pubblication in October 2000, publication foreseen in 2001)

609. Salvi M. (2001) 'To what extent should animal cloning be permitted?' EJIAIB Journal of Asian and International Bioethics (in press, n. 1, 2002)

610. Salvi M. (2001) 'Transforming Animal Species: the Case of 'Oncomouse', Ethics and Engineering Life (7), Boston-London: MITpp. 15-28

611. Sandoe P. \& Holtug N; (1993) 'Transgenic animals' Livestock production science (36):113-116

612. Sandoe P. (1999) 'Ethical perspective on breeding and biotechnology in the future developments' in Farm animal breeding and reproduction and its. ethical, legal and consumer implications, report to the European Commission EC-ELSA; November 1999

613. Schlick M. (1961-62) Causality in Contemporary Physics' British Joumal for 
the Philosophy of Science (12) pp. 177-193;281-298

614. Schwartz S. (1981) 'Natural kinds' Behavioural and Brain Sciences (4) pp. 301-302

615. Scott G. E. (1990) Moral Personhood . Albany: State University of N.Y. Press NY

616. Searle, J. R. (1992). The Rediscovery of the Mind. Cambridge, MA : MIT Press

617. Sedlack B. (1999) 'Transgenic mousc models' Genetic Engineering News (19) November 1 p. 14

618. Seife C. (1999) 'Without Consent' New Scientist 13 November 1999:49-51

619. Sgreccia E. (1986) Manuale di Bioetica Milano Vita e pensiero

620. Shapiro H. (1999) 'Ethical consideration and public policy: A ninety day exercise in practical and professional ethics: cloning human beings' in Science and engineering ethics 5 (1) pp. 3-16;

621. Shea B. \& Sitter (1989) Scientist and their responsibility Watson Publishing International

622. Shepard G. (1983) Neurobiology Oxford University Press N.Y.

623. Shepard R (1962) 'Analysis of proximitics: multidimensional scaling with an unknown distance function III' Psychometrica (27) pp. 125-40

624. Sheppard R. (1979) 'Additive clustering' Psychological Review (86) pp. 87123

625. Shewmon D. (1988) 'Anenchephaly Selected Medical Aspccts' Hasting Centre Report 18 (5) pp. 11-19

626. Shiclds P. et all. (1999) 'Analysis of teleomere lights in cloned sheeps' Nature (399):316-317

627. Simpson G. (1961) Principles of Animal Taxonomy Columbia University Press N.Y.

628. Simpson G. (1967) The meaning of evolution Yale University Press New Haven

629. Singer M. \& Breg. P. (1991) Genes and Genomes University science Books Mill Valley CA

630. Singer P. \& Wells D. (1994) 'Genctic Engineering' in Ethical Issues in Scientific Research: An Anthology Erwin E. eds. Garland Publishing New York pp. $307-320$

631. Singer P. (1985) In Defence of Animals N.Y.: Blackwell

632. Singer P. (1990) Animal Liberation N.Y.: Avon Books

633. Singer P. (1993) Practical Ethics 2 nd ed. N.Y.: Cambridge University Press

634. Singer P. (1994) Rethinking Life \& Death Text Publishing Company Melbourn

635. Singer P. et al (1990) Embryo Experimentation Cambridge University Press

636. Smaglick P. (1999) 'Animal studies boost gene theraphy vector's prospects' The scientist $13(8): 9$

637. Smaglik P. (1999) 'Monkey/human chimera gaining favour in AIDS research' The Scientist, August 30, 1999:13

638. Snowdon P. F. (1991) 'Personal Identity and Brain Transplants' Human Beings ed. David Cockburn. Cambridge: Cambridge University Press. 
639. Snowdon P. F. (1995) 'Persons Animals and Bodies' The Body and the Self ed. Bermúdez J. Marcel A. \& Eilan N. Cambridge MA: MIT Press 1995 pp. $71-86$.

640. Sober E. (1984) The Nature of Selection MIT Press Cambridge Mass.

641. Sober, E. \& Wilson, D. S. (1998). Unto Others: The Evolution and Psychology of Unselfish Behavior. Cambridge, MA: Harvard University Press.

642. Sokal R. (1963) Principles of numerical taxonomy Freeman San Francisco

643. Soltor D. (1987) 'Inertia if the embryonic genome in mammals' Trends in Genetic: 3 pp. 23-27

644. Spier R. (1999) 'An approach to the ethics of cloning humans via an examination of the ethical issues pertaining to the use of any tool' in Science and engineering ethics 5 (1) pp. 17-33.

645. Spom O. Recke G. \& Edelman G. (1989) 'Reentrant Signalling Among Simulated Neuronal Groups Leads to Coherency in their Oscillatory Activity' Proceedings of the National Academy of Science (86) pp. 7265-69

646. Stanbury J. (1983) The Metabolic Basis of inherited disease N.Y Mc-Grow-Hill

647. Stanley H.D. (1994) 'Statistical mechanics in biology: how ubiquitous are longrange correlations?' Phisica (293) pp. 244 - 253

648. Stebbins. G. (1969) The Basis of progressive evolution University of North Carolina Press Chape Hill

649. Stein D. 'Dissipative structures broken symmetry and theory of equilibrium phase transition' J. Chem. Phys. (72) pp. $2869-2874$.

650. Steinbock B (1992) Life before Birth. The moral and Legal Status of Embryos and Fetuses Oxford University Press N.Y. Oxford

651. Stemberg R.J. (1990) Metaphors of the Mind Cambridge University Press Cambridge

652. Stent (1974) 'The dilemma of science and morals' Genetics' pp. 41-51;

653. Stern M. (1999) 'Novel vectors for gene therapy' Genetic Engineering News, January 1999 pp. 28

654. Stewart I. \& Golubtsky M. (1992) Fearful Symmetry: is God a geometer? Blackwell Oxford

655. Stock G. \& Campbell J. eds., (2000) Engineering the Human Germline: An Exploration of the Science and Erhics of Altering the Genes We Pass on to Our Children Oxford and New York: Oxford University Press

656. Strawson, G. (1997). The self. Journal of Consciousness' Studies, 4(5/6), 405 428.

657. Strebbins G. (1982) 'Plant in Speciation' Barigozzi eds. Mechanisms of Speciation Alan R. Liss N.Y. pp. 21-39

658. Sultans S. (1987) 'Evolutionary implications of phenotypic plasticity in plants' Evolutionary Biology (21) pp. 127-178

659. Sussman M. et al. (1988) The Release of Genetically Engineered Microorganisms Academic Press N.Y. 
660. Sutton A. (1995) 'The New Genetics: Facts, Fictions and Fears' Linacre Quarterly 62(3) pp. 76-87

661. Suzuki D. \& Knudson P. (1987) Genethics, the ethics of engineering life Stoddart Publishing Co. Toronto

662. Swinburne R. (1984) 'Personal identity, the dualist theory' in Shoemaker eds. (1984)

663. Szallai Z. (1998) 'Gene expression patterns and cancer' Nature Biotechnology (16) pp. 1292-1293

664. Tauber A. (1992) Organisms and the origin of Self Kluwer Academic Publisher Dordrecht/Boston/london

665. Tauer C. (1990) 'Does Human Gene Therapy Raise New Ethical Questions?' Human Gene Therapy 1(4) pp. 411-418

666. Tauer C. (1995) 'Human growth hormone: a case study in treatment priorities' Hastings Center Report. 25(3.) 1995 pp. 18-20.

667. Taylor C. (1989) Sources of the self Cambridge Cambridge University Press

668. Taylor R. (1998) 'Superhumans' New' Scientist, 3 Octobcr, pp. 25-29

669. Temple L. K. F. et all. (2001) 'Defining disease in the genomic era' Science (29.3):807

670. Terrace H.S. (1979) Nim A Chimpanzee Who Learned Sign Language Random House N.Y.

671. Terragni F. (1999) /l Clone e il suo doppio, Milano, Mondadori

672. Terrgni F. (1984) I/ codice manomesso Milano Feltrinelli

673. The future developments in farm animal breeding and reproduction and their ethical, legal and consumer implications (1999) Report to the European Commission DG RTD

674. Thomas L. (1987) 'The hazards of science' New England Journal of Medicine (296) pp. 324-328

675. Thomas S. (1998) 'The ethics of mental disorders and genetics' SPA:18-19

676. Thompson L. (1993) 'Should Dying Patients Receive Untested Genctic Methods?' Science 259(5094) pp. 452

677. Thompson L. (1994) Correcting the Code: Inventing the Genetic Cure for the Human Body. Simon \& Schuster New York

678. Thompson M.W. (1980) Genetics in Medicine 3rd Edition Suunders Company Philadelphia London. Toronto

679. 'Thompson P. (1997) 'Ethics and the genetic enginecring of food animals' Journal of agriculture and environmental ethics $10(1): 1-23$

680. Thompson, E. (1995). Colour vision: $A$ study in cognitive science and the philosophy of Perception. London: Routlcdge.

681. Thomson J.A. (1998) 'Embryonic Stem Cell Lines Derived from Human Blastocysts' Science (282):1145-1147

682. Tiezzi E. (1984) Tempi Storici, Tempi Biologici Milano Garzanti

683. Toates F. (1986) Motivational Systems Cambridge Cambridge Univesity Press

684. Toates F. (1995) Stress Conceptual and Biological Aspects Chicester 
685. Tononi G. \& Edelman G.M. (1999) 'Consciousness and complexity' Science (282):1846-1851

686. Trifonof E. (1989) 'View point: the multiple codes of nucleotide sequences', Bul. Math. Biol. 51 pp. $417-432$

687. Tsukui T. et al. (1996) 'Transgenesis by adenovirus-mediated gene transfer into mouse zona-free eggs' Nature Biotechnology (14) pp. 982-5

688. Turchin \& Taylor (1990) 'Complex dynamics in ecological time series' Theoretical Population Biology (38) pp. 1-57

689. Turing A. (1952) 'The chemical basis of morphogenesis' Philosophical Transactions of the Royal Society of London (237) pp. 37-72

690. Turner D. (1970) The autonomous man: an essay in personal identity and integrity St. Louis: Bethany Press.

691. Tye, M. (1995). Ten Problems of Consciousness. Cambridge, MA: MIT Press.

692. Unger P. (1990) Identity consciousness and value. N.Y. Oxford University Press.

693. Van De Beer D. (1983) 'Interspecific Injustice and Animal Slaughter' Williams W, eds. Ethics and Animals Humana Press Clifton N.Y. pp. 147-162

694. Van den Bos et al. (1997) Animal Consciousness and Animal Ethics Van Gorcum \& Comp. Assen

695. Van Valen L. (1978) 'Evolutionary Species Concept Reconsidered' Systematic Zoology (27) pp. 17-27

696. Van Vleck (1962) Encyclopaedia Britannica vol. XVIII Chicago pp. 827-828

697. Varela F. (1991) 'Organism: A Meshwork of Selfless Selves' Organism and the Origins of Self Dordrecht/Boston/London Kluwer Academic Press

698. Verhog H. (1999) 'Bioethics and the intrinsic value of animals' in Doll et all. (1999):72-81

699. Verlier F. (1999) 'La toxicologie de la therapie genique' Biofutur (188):31-35

700. Vines G. (1998) 'Hidden Inheritance' New Scientist, November 1998, pp. 2630 ,

701. Vogel G. (1999) 'Chimps in the wild shows stirrings of culture' in Science $(284): 2070-2073$

702. Vogel G. (1999) 'Harnessing the power of stem cells' Science (283):1432-1433

703. Vogel G. (2000) 'Company gets rights to cloned human embryos' Science (287):559

704. Vogel G. (2000)'Capturing the promise of youth' Science (286):2238-2239

705. Von Sconberg (1995) Ethics Risks and Public debate Tilburg Un. Press

706. Vyacheslav L. Kalmykov (1999) The Generalized Theory of Life Unified Understanding of Complexily (Organization, Wholeness) \& Self-Organizing (Evolution) in http://www.iteb.serpukhov.su/GENTL/

707. Vygtoskji L. (1974) Storia dello sviluppo infantile e delle funzioni psichiche superiori tr. it. Firenze Giunti Barbera

708. Wachbroit R. (1997) Genetic Encores: The Ethics of Human Cloning Report for the Institute of Philosophy and Public Policy 
709. Waddington C. H. (1977) Tools for thought the Estate of the late C.H. Waddington Edindurgudh

710. Wadman M. (1998) 'NIH launches discussion of in utero gene therapy' Nature vol. 393, October 1998:420

711. Wagner G. (1988) 'The Influence of Variations of Developmetal Constrains on the Rate of Multivariate Phenotype Evolution' Journal of Evolutionary Biology (1) pp. 45-66

712. Wagner G. (1994) 'Homology and the Mechanisms of Development' AA.VV. The Hierarchical Basis of Comparative Biology Yale Academic Press

713. Wagner G. (1996) 'Homology Hox genes and Developmental Integration' American Zoology (36)1996 pp. 4-13

714. Wagner G. et al. (1989) 'Are in vitro fertilisation and embryo transfer of benefits to all?' Lancet (28 Oct.) pp. 1027-1029;

715. Wagner W.H. (1969) 'The construction of a classification' Systematic Biology National Academy of Sciences Washington D.C.

716. Waismann F. (1961) 'the Decline and Fall of Causality' Crombie C. Turning Points in Physics N.Y.

717. Wakayama (1998) 'Clone Watching' New Scientist May 1998 pp. 35

718. Walker S. (1983) Animal Thought Routledge \& Jegan London

719. Walters L. (1986) 'The ethics of human gene therapy' Nature (320) pp. 225 227.

720. Walters L. (1991) 'Ethical issues in human gene therapy' Journal of Clinical Ethics. 2(4) pp. 267-274.

721. Walters L. (1991) 'Human gene therapy: ethics and public policy' Human Gene Therapy (2) pp. 115-122.

722. Walters L. (1999) 'Ethical Issues in human gene therapy' Human genome News $10(1-2) 16-17$

723. Warkany J. (1971) Congenital Malformations Chicago Year Book Medical Publisher

724. Wannok M (1983) 'In vitro fertilisation: the ethical issues (II)'in The Philosophycal Quarterly (33)

725. Warnok M. (1987) 'Do human cells have rights?' Bioethics (1) pp. 10-11

726. Watson (1981) The DNA Siory Freman San Francisco

727. Watson J. (1998) 'Good genc, bad gene' Time Special lssue 'The new age of discovery' Winter 1997-1998, pp. 86-88

728. Weiss R. (1990) 'Growth-gene Mickey makes mice mini' Science News (138) pp. 20

729. Weiss R. (2000) "Dolly's Premature Aging Not Evident in Cloned Cows," The Washington Post, (April 28, 2000), p. A3

730. Wertz D. (1997) 'Cloning Humans: is it Ethical?' Gene Letter /lssue 5 March

731. West Germany. Enquête Commission. 'An Extract from Prospects and Risks of Gene Technology pp. The Report of the Enquête Commission to the Bundestag of the Federal Republic of Germany' Bioethics 2(3) pp. 254-275, July 1988 
732. White G. B. (1993)'Human growth hormone: the dilemma of expanded use in children' Kennedy Institute of Ethics Journal, 3(4) pp. $401-409$.

733. White M. (1978) Modes of Speciation San Francisco Freeman

734. WHO, CIOMS (1990) The Declaration of Inuyama on Human Genome Mapping, Genetic Screening and Gene Therapy

735. Wiepkema P. (1997) 'The emtional vertrebate' in den Bos eds. pp. 93-103

736. Wiggins D. (1980) Sameness and substance Basil Blackwcll Oxford

737. Wiggins D. (1987) 'The Person as Object of Science As Subject of Experience and as Locus of Value' Persons and Personality ed. A. Peacocke \& G. Gillett, Oxford: Blackwell..

738. Wiggins D. (1987) 'The person as object of science, subject of experience locus of value' in Peacocke \& Gillet eds. Persoins and personality Blackwell Oxford

739. Wiggins D. (1987) Needs Values Truth: Essays in Philosophy of Value Blackwell Oxford

740. Wilckelgren I. (1999) 'Nurture helps mold able minds' Science (283) pp. 1832 1834

741, Wiley E. (1981) Phylogenetics: The Theory and Pratice of Philogenetics Systematics John Wiley and Sons N.Y.

742. Wilkie T. (1993) Perilous Knowledge The Human Genome Project and its Implications London Faber \& Faber Press

743. Williams B. (1970) 'Are Persons Bodies?' The Philosophy of the Body ed. S. Spicker. Chicago: University of Chicago Press.

744. Williams B. (1973) Problems of the self Cambridge Univeristy Press

745. Williams M. (1979) Brain Damage Behaviour and the Mind Wiley N.Y.

746. Williamson M. et al. (1986) 'Research need the reduction of several disease' Human Embryo Research Yes or No? Foundation Symposium London Tavistock pp. 105-121

747. Wilson B. (1991) 'Are Species Sets?' Biology and Philosophy (6) pp. 413-431

748. Wilson B. (1995) 'The Species Problem' Biology and Philosophy (12) pp. $339-$ 356

749. Wilson E. O. (1975) Sociobiology: The new synthesis Cambridge Mass. Harvard University Press

750. Wilson J. (1999) Biological Individuality Cambridge University Press Cambridge

751. Wilson, B. E. (1996) 'Changing conceptions of species' Biology and Philosophy 11:405-20

752. Wimsatt W.C. (1972) 'Teleology and the logical structure of functions statements' Studies of philosophy of science pp. 1-80

753. Windsor, M.L. and Hutchinson, P. (1990), "The potential interactions between salmon aquaculture and the wild stocks - a review." Fisheries Research 10, pp. $163-176$

754. Winger E. (1970) 'Epistemology and Quantum Mechanics' Symmetry and Reflections MIT Press Cambridge Mass. 


\section{SUMMARY}

This dissertation addresses the notion of individuality in both biology and philosophy. This topic is not new. Biologists, philosophers of science and bioethicists have often explored the complexity of the above-mentioned topic. What is new is an issues that brings together a number of scientific disciplines (from neuroscience to molecular biology, from genetics to biological systematics, and so on) to claim a theory that refers to what we do know about living beings' biological existence. This analysis across different branches of the Natural sciences is enriched by the philosophical interpretations and explanations of the individuality problem in order to advocate a theory, based on science and philosophy, that explains living beings' individuality as biological and psychological entities.

In parallel the dissertation addresses issues related to the use of biotechnology in producing genetically manufactured organisms (GMOs, transgenic animals, chimeras, and so on). At the present time, biotechnology can modify the biological source of living beings. Cloning, germline gene transformations and the production of chimeras, are feasible. Technical problems are being solved. Nevertheless, every growth of the human knowledge needs to be implemented by critical studies on the right way to use the available discoveries. History teaches us that a reflection on the potentialities of new technologies is a pre-requisite for their use. This will help to avoid unexpected consequences or simply to prevent a cascade of events transforming potential benefits in unexpected negative consequences. 
Thus, to analyse problematic implications arising from the use of new technologies is an urgent need of modern society. Accordingly in this dissertation I address case studies that are being debated among biologists and bioethicists from ANDi to Dolly, from the oncomouse to the human-cow hybrid. The above mentioned case studies have a common factor: they change the biologic features of future-born organisms. They not only change the natural reproductive mechanisms, as other new reproductive techniques do (such as in vitro fertilisation, assisted reproduction, etc.), they also change the genetic makeup of individuals at a certain time and trough time.

In the following sections I will present a summary of the various chapters of the thesis. The thesis has two parts. The first part deals with biological and philosophical problems involved in the notion of individuality. The second part deals with issues related to the use of biotechnology to genetically modify future born organisms' genes. 


\section{Summary of the chapters of the thesis}

\section{Sections One: Biological Problems}

In the first two chapters of the thesis the notion of biological individuality is analysed from the point of view of theoretical biology and analytic philosophy. The chapters advocate (a) the limits of genetic mechanistics and (b) the need to use to non-reductionistic scientific theories (from organismic biology to complexity theory) to explain the biological and philosophical complexity living beings' individuality. A theoretical model (the organic unity theory) is here advocated. According to the organic unity theory organisms are complex entities persisting over time. Therefore it is not possible to claim the existence of a biological factor having a basic value towards the existence of living beings. But it is possible to find causal mechanisms of unification of the biological heterogeneity of living beings (cohesive factors) in a unitary structure that varies over time while persisting. The fundamental property of the organic unity concept is that an organic unity cannot be explained via the sum of its constituents. This condition is respected in biology because the natural phenomena portray that the coexistence of biological systems, and their cohesive integration, causes the 'emergence' of characters. This cannot be deduced from knowledge of a specific organism's constituents. The unitary meaning of biological systems allows us to understand their emergent properties. The organic unity model here proposed intends to answer a number of specific questions, such as: "what is a biological individuality?', 'is it possible to reduce 'individuality' to genes?', 'what element diversifies a whole of cells from an individuality?' 
The above mentioned topics are also addressed in the third chapter of the thesis through systematic biology (natural taxonomy). This is to address the notion of individuality at a species level. Following the interdisciplinary approach used in the previous chapters, the species concept is explored from a biological and philosophical viewpoint, by looking at the clustering mechanisms that correlate species-specific organisms and differentiate them from individuals belonging to other species. The organic unity model is applied to biological taxonomies and confronted with other paradigms claimed in systematics biology. This comparative study aims to stress the limits reductionistic approaches used in biology and approach the relationship between environment and organism. The results arising from such an analysis show that the diachronic and interactive nature of organisms limits the use of mathematical models in biology (both in systematic biology and evolutionary epistemology).

In the other chapters I study the psychological nature of individuals. Therefore I approach the notion of individuality through the viewpoint of neuroscience. On the one hand, I stress the biological nature of the mind, and on the other hand, I analyse a number of theories based on reductionism (quantum theory applied in neuroscience). The main result of such an approach is that the mental existence of living beings is hardly explainable through neural physics and the mind problem has to be addressed through biology. To complete such an analysis the sixth chapter examines theories advocated by neuroscientists and philosophers to explain the mental phenomena of individuals (memory, self-awareness, selfsynthesis, and so on). By using an interdisciplinary approach, and stressing specific case studies (from people suffering of psychological dysfunctions to anencephalics), I underline the 
limits of reductionistic explanations of the mind. The main questions faced are 'to what extent would the psychological criterion explain the notion of individuality?', 'what is the role of self-awareness and self-reflexivity with regard to the unification of the Self?'

While the above-mentioned chapters show the need to explain the diachronic nature of living beings, the seventh chapter approaches issues related to the persistence of living beings through time. It examines philosophical explanations (the psychological criterion) advocated by philosophers to explain the notion of individuality of living beings with particular emphasis to their persistence over time. I stressed the need of advocating an explanation of both the psychological and the biological variability of living beings. I then use a conceptual model (the closest continuer theory) advocated by R. Nozick to elucidate the continuity of subjects as biological and psychological entities.

In order to check whether the above mentioned model is applicable to both the biological and psychological features of an individual (at a specific time and trough time), a specific chapter is then devoted to apply the organic unity theory to both the mental and biological existence of living beings. It identifies analogous processes that unify individuals through time and at certain times. It shows that the biological and psychological clustering mechanisms have a common goal: to unify the biological and psychological features of living beings in a unitary entity that persists over time while varying.

Finally, because one of the non-biological clustering process that unifies both the species and the individual is culture, I then address the role of cultural traits towards the unification of the species and the synthesis of an individual's Self. 
To summarise, the basic conclusion of the first section of the thesis is that individuals are organic units, they are loci of integrated biological-psychological complexity unified in a heterogeneous and cohesive unitary dimension that varies over time by adapting to environmental variables.

\section{Sections Two: Ethical issues}

I begin the second section of the thesis by explaining reasons why the problem of individuality is applicable to discuss the ethics of biotechnology. Contrary to the common approach used by a number of bioethicists dealing with biotechnology, I claim that studies focusing on the safety and effectiveness of biotechnologies do not provide valid arguments to claim their ethical legitimacy. I also explain reasons why the organic unity theory can be used to discuss ethical issues arising from those applications of biotechnology that involve the modification of living beings' germ cells (thus affecting both the genetic and phenotypic identity of future born individuals).

Having clarified the link between the organic unity theory and bioethics, I then report the state of the art of medical applications of human genetics taking into account the implications of the announcement of the sequencing of the human genome (e.g. proteomics). The second chapter addresses the concept of genetic disease, the results achieved with gene therapy and the implication of genetic reductionism towards gene therapy. The main conclusion of the chapter is that from a biological point of view a number of pro-arguments to support gene therapy fail due the state of the art of human genetics (effectiveness, safety and so on). I then discuss arguments provided by bioethicists to oppose or accept human germ line 
gene manipulations. The study shows that, contrary to the public perception and the regulatory framework adopted at a world level, none of the arguments claimed to discuss the ethical legitimacy of human germ line gene modification provide final justification to accept or reject such an application of human biotechnology. I then propose an alternative approach to the topic above based on the organic unity theory. This approach claims that human germ line gene manipulations will certainly affect the biological persistence of future born individuals and as such as a negative connotation in itself. However, this preliminary consequence should be related to a precise positive outcome (a successful treatment) to allow us to say whether the expected therapeutic outcomes are met. To date, somatic applications of gene therapy show that this technology is not safe and effective. Then we do not have solid reasons to claim that clinical trials involving gene modification of human beings' germ cells should be performed. This also with a clear awareness of existing contradictions that show that while human germ line gene manipulations is forbidden by law, concrete examples of transmissible gene modifications exist with mitochondria disease therapy.

As the human genome sequencing has showed, the genetic differences between animal species are minimal. I then approach the issue to genetically modify future born in animal biotechnology (transgenic animals). On the one hand I report the state of the art of animal biotechnology and analyse arguments provided by advocates of animal rights (animal welfare) to discuss the ethics of animal technology. On the other hand I apply the above arguments to the production of transgenic animals for research purposes. I address a specific example 'the oncomouse'. I use the organic unity theory to discuss the ethics of producing transgenic animals. Contrary to 
the position advocated by animal rights proponents (as well advocates of biocentric ethics) I do not see arguments to avoid research applications with animals when protocols of good research practices are followed and clear scientific goals are expected from the trials. I claim that an ethical taxonomy is defensible trough the organic unity view. And this is also if the identity issues would negatively connote such an application of animal biotechnology.

In the final two chapters of the second section of the thesis 1 deal with human and animal applications of Nuclear Transfer Technologies, better known as reproductive cloning. I first explore the state of the art of cloning by stressing both the biological implications of such a technology (safety, effectiveness etc.) and its philosophical implications. I focus on issues related to animal cloning, and I claim that from a philosophical viewpoint arguments to support animal cloning are weak. Then, I claim that there are no arguments that, from an ethical viewpoint, may advocate the ethical legitimacy of animal cloning performed in animal breeding programmes. This is because the expected goals of animal cloning seem not to have a sufficient validity to justify the consequences that clones undergo. To conclude, I then face human reproductive cloning, one of the most controversial applications of biotechnology, Having already analysed the biological implications of cloning, I completely focus on the ongoing debate about its ethical legitimacy. I analyse the pro-cons arguments claimed by bioethicists to oppose-accept human reproductive cloning, as well as the identity paradoxes arising from such a technology. The main result of such an inquiry is that from a philosophical point of view no final arguments have been posited to oppose or accept human cloning. The main problem is the expected outcome that may arise from such a 
technology. At the present there are no applications that may justify human reproductive cloning although there are also no arguments to refute it. 


\section{CURRICULUM VITAE}

Maurizio Salvi was born in Tropea, a small village in the South of Italy, in 1967. He has a MBA in Modern Literature (University of Rome, Faculty of Literature, 1985-1991), a MBA in Philosophy (University of Rome, Faculty of Philosophy, 1991-1995), a MD in Bioethics (University of Rome, Faculty of Natural, physical and mathematical sciences, 1995). He has done research on ethics and biotechnology at the University of Rome (1994-1999), the Flemish Institute of Biotechnology (1999-), the University of Maastricht (WTMC Institute -1996-1998), the International Forum of Biophilosophy (1998-) and the Higher Institute of Philosophy, University of Leuven (1998-1999). He was the Director of the Bioethics Course at the Inter-University Centre Dubvronik (1999-2001). Since 1998, he is working as a Scientific Officer at the European Commission, (Directorate -General Research) responsible for Bioethics research in Europe.

He has published extensively on ethics and biotechnology and philosophy of biology in Europe, USA, Japan, South America and New Zealand. He is an honorary member of the European Association of Centre for Medical Ethics (EACME), and a member of the following professional and academic associations: European Society on Agriculture and Food Ethics (EuSsafe); Associazione Italiana di Biologia Teorica (ABT); International Society for the History, Philosophy \& Social Studies of Biology (ISHPSSB); Asian and International Bioethics Association (AIBA), 'genetics and bioethics network', International Association of Bioethics (IAB), the American Association for the Advancement of Science (AAAS). 


\section{Publications resulting from the Thesis.}

The entire Dissertation has been submitted for publication to the Cambridge University Press, London (book series on philosophy and biology). The Editor has accepted the project and the peer review process (both in EU and US) is ongoing. To date, I did not get the final decision from the peer review committee yet.

Papers published are the following:

- Salvi M. (1995) 'Epistemologia biologica e bioetica evoluzione molecolare e biotecnologie' Methodologia thought language and methods. Review of the Italian National Research Centre (14 December 1995 pp.33-51).

- Salvi M. (1995) 'Ethics and biotechnology, analysis of the relation between ethics and science' EJAIB Journal of Asian and International Bioethics (7) November 1995 pp. $151+3$.

- Salvi M. (1996) 'Biotecnologie e terzo mondo un problema di giustizia' Qualita' Equita' Rivista del welfare del futuro Editrice Libereta' S.r.l. n.3 September pp. 111-123.

- Salvi M. (1996) 'La responsabilita' della scienza' Bioethics and Humanities University of Rome la Sapienza n.2 Internet 1996 (http://www.uniromal.it/bioetica/home.htm)

- Salvi M. (1996) 'Lo specchio e il labirinto filosofia analitica e identità personale' Prometeo. Rivista trimestrale di scienze e storia Milano Mondadori ed. Anno $13 \mathrm{n} .51 \mathrm{pp}$. 78-88. 
- Salvi M. (1996) 'Morte Cerebrale e Problemi di Identita'; la filosofia analitica del XX secolo e il problema della morte del soggetto' Bioethics and Humanities University' of $\begin{array}{lll}\text { Rome n.3 Internet, } & 1996\end{array}$ (http://www.uniroma1.it/bioetica/home.htm)

- Salvi M. (1997) How Calculating the Utility of Human Germline Gene Transformations? A critic to the Utilitarianism in Bioethics'EJAIB Journal of Asian and International Bioethics (7) March 1997 pp. 36-38

- Salvi M. (1997) 'La Biologia come scienza nonparadigmatica' Methodologia thought language and methods Review of the Italian National Research Centre n. 17 January 1997.

- Salvi M. (1997) Paradossi Biotecnologici: Identita' Personale e Genetica Applicata' Bioetica rivista interdisciplinare 21997 Milano Franco Angeli eds. pp. 171-192

- Salvi M. (1998) 'Human Genetics and the integrity of Self: Germline Gene Therapy' in D. Macer \& Fujiki N. eds. Bioethics in Asia Cristchurch NZ 1998 pp.278-284

- Salvi M. (1998) 'Ontology and Bioethics, the Case of Human Dignity principle in Human genetics' EJAIB Journal of Asian and International Bioethics 8 (6) November pp. 181-183

- Salvi M. (1998) Ethics and Germ-line Manipulations: Germ-line transformation of the transgenic mouse, Scientific Report to the European Commission, Programme: Biotechnology

- Salvi M. (1999) 'Casualita' Biologica Causalita' Biotecnologica' in Bioetica e professione medico veterinaria Quaderni di Bioetica. July 1999 Bologna Macro Edizioni, pp. 109-134 
- Salvi M. (1999) 'Organismi ed Individualità, Controversie sul concetto di individuo in biologia' Prometeo. Rivista trimestrale di scienze e storia Milano Mondadori

- Salvi M. (2001) 'Biotecnologie e bioetica, un ritorno alla metafisica? Terapia genica in utero, clonazione umana e lo statuto morale dell'embrione.' Bioetica, rivista disciplinare, Roma (foreseen 2002)

- Salvi M. (2001) "Complessità e bioetica. La bioetica nelle istituzioni europee' Keiron Roma Farmaindustria pp. 170175

- Salvi M. (2001) 'Genetics' Dreams in the post-genomics era' Medicine, Health Care and Philosophy Kluwer Academic Publisher (in press)

- Salvi M. (2001) 'Multiplying individuals, the human reproductive cloning', Ethics and Politics Università di Trieste (in press)

- Salvi M. (2001) 'Shaping Individuality, Ethics and Human Inheritable Germ Line Gene Modification' Theoretical Medicine Kluwer Academic Publisher (accepted for pubblication in October 2000, publication foreseen in 2001)

- Salvi M. (2001) 'To what extent should animal cloning be permitted?' EJAIB Journal of Asian and International Bioethics (in press, n. 1, 2002)

- Salvi M. (2001) 'Transforming Animal Species: the Case of 'Oncomouse', Ethics and Engineering Life (7), BostonLondon: MIT pp. 15-28 